\title{
Measurement of the Calorimetric Energy Scale in MINOS
}

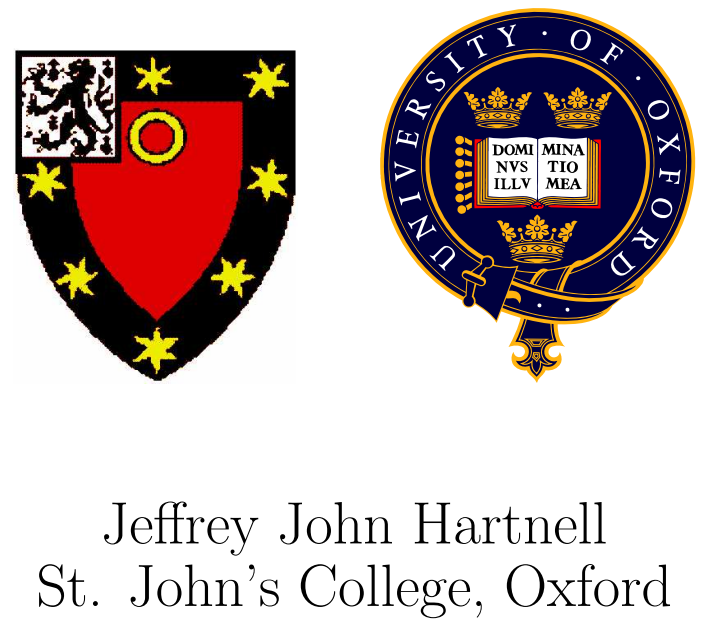

Thesis submitted in fulfilment of the requirements for the degree of Doctor of Philosophy at the University of Oxford

Trinity Term, 2005 


\title{
Measurement of the Calorimetric Energy Scale in MINOS
}

\author{
Jeffrey John Hartnell \\ St. John's College, Oxford \\ Thesis submitted in fulfilment of the requirements for the degree of \\ Doctor of Philosophy at the University of Oxford. \\ Trinity Term, 2005
}

\begin{abstract}
MINOS is a long-baseline neutrino oscillation experiment. A neutrino beam is created at the Fermi National Accelerator Laboratory in Illinois and fired down through the Earth. Measurements of the energy spectra and composition of the neutrino beam are made both at the source using the Near detector and $735 \mathrm{~km}$ away at the Soudan Underground Laboratory in Minnesota using the Far detector. By comparing the spectrum and flavour composition of the neutrino beam between the two detectors neutrino oscillations can be observed. Such a comparison depends on the accuracy of the relative calorimetric energy scale.

This thesis details a precise measurement of the calorimetric energy scale of the MINOS Far detector and Calibration detector using stopping muons with a new "track window" technique. These measurements are used to perform the relative calibration between the two detectors. This calibration has been accomplished to $1.7 \%$ in data and to significantly better than $2 \%$ in the Monte Carlo simulation, thus achieving the MINOS relative calibration target of $2 \%$.

A number of cross-checks have been performed to ensure the robustness of the calorimetric energy scale measurements. At the Calibration detector the test-beam energy between run periods is found to be consistent with the detector response to better than $2 \%$ after the relative calibration is applied. The muon energy loss in the MINOS detectors determined from Bethe-Bloch predictions, data and Monte Carlo are compared and understood.

To estimate the systematic error on the measurement of the neutrino oscillation parameters caused by a relative miscalibration a study is performed. A $2 \%$ relative miscalibration is shown to cause a $0.6 \%$ bias in the values of $\Delta m^{2}$ and $\sin ^{2}(2 \theta)$.
\end{abstract}


To my parents. 


\section{Acknowledgements}

During my years as a graduate student I have had the rather unusual experience of having 3 supervisors at one time or another. Many thanks must go to Alfons Weber, Geoff Pearce and Peter Litchfield. I have really enjoyed working with them all and very much appreciate the guidance they gave me. Of my 3 supervisors Alfons has certainly devoted the most time to my work. His quick and deep understanding of the problems I encountered in my research, together with his enthusiasm and many good ideas (for more plots!) made him an excellent supervisor. At the Rutherford Appleton Laboratory, Peter was my original supervisor before reaching retirement age at RAL and then taking up a Professorship at the University of Minnesota. Geoff took over my supervision at RAL in 2002 and he has taught me a great deal. I am indebted to him for his advice and moral support over the years, which have proved very valuable.

During my last year I had the fortune to share an office with Nathaniel Tagg. His interest in my work, willingness to answer many questions, as well as his advice and general approach towards experimental physics has taught me a lot. David Petyt should also get a special thanks for introducing me to neutrino physics and MINOS in general when I first started. Thanks also to Bill Scott for the general support at RAL and discussions of neutrino physics theory.

My "partner in crime" when it came to MINOS was Anatael Cabrera. Since starting together in 2001 we have shared the graduate student experience at Oxford, CERN and all over the US. My friend Sageet summed Anatael up by saying he was a guy with a "big heart". Anatael also has a wicked sense of humour, which I have had the pleasure of sharing in at many, many dinners, lunches and outings.

At Oxford and RAL it's also been a pleasure to work with Wade Allison, Costas Andreopoulos, Matthew Barker, Giles Barr, Tass Belias, John Cobb, Antonella De Santo, Mark Dorman, Tim Durkin, Justin Evans, Katarzyna Grzelak, Annalisa L'abbate, Phill Litchfield, Paul Miyagawa, Tim Nicholls, Tobias Raufer and Nick West.

My four months at the Soudan Underground Laboratory wouldn't have been nearly as enjoyable if it wasn't for Alex Sousa, Jen Westland, Heather Hanily, Jeremy Gogos, Emily Maher, Dipu Rahman, Ryan Nichol, Jeff Nelson, Bill Miller, Earl Peterson and everyone who came for a week or two.

Thanks to everyone who helped make the 2003 Calibration detector experiments happen: Jenny Thomas, Karol Lang, Mike Kordosky, Tricia Vahle, Phil Adamson, Ruben Saakyan, Gordon Crone, Leo Jenner, Chris Smith, everyone from Oxford and RAL, as well as everyone involved in getting the Near detector intrumentation up and running.

Life at Oxford wouldn't have been nearly as much fun without the students of St. John's Middle Common Room. Thanks to everyone for the bops, the guest dinners, the garden 
parties, the coffee after lunch in Tommy White quad and all the rest. In some sort of chronological order, thanks to Sophie Lamle, Mike Froggatt, Sageet Amlani, Lizzie Macaulay, Lisa Lazareck, Rakhee Patel and Mark Harris for making life at St. John's so fantastic.

The loving support and encouragement of my parents, sister and grandparents has been totally invaluable over the years and without whom I would not be where I am today; many thanks to them. Finally, a very special thanks to Sharon Cuthbert for her love, support and patience over the past year. 


\section{Contents}

1 Introduction $\quad 1$

2 Neutrino Physics $\quad 4$

2.1 The "Little Neutral One" - A Brief History . . . . . . . . . . . . . . . . . 4

2.2 Neutrino Mass and Oscillations . . . . . . . . . . . . . . 6

2.2 .1 Neutrino Oscillations . . . . . . . . . . . . . . . . . 7

2.2 .2 Matter Effects . . . . . . . . . . . . . . . . 12

2.3 Experimental Evidence for Neutrino Oscillations . . . . . . . . . . . . . 13

2.3.1 Solar Neutrinos . . . . . . . . . . . . . . . . . . . . . 13

2.3.2 Atmospheric Neutrinos . . . . . . . . . . . . . . 22

2.3.3 Reactor and Accelerator Neutrinos . . . . . . . . . . . . . 30

2.3 .4 Summary . . . . . . . . . . . . . . . . . 31

2.4 A Look to the Future . . . . . . . . . . . . . . . . . . 31

3 The MINOS Experiment 33

3.1 The NuMr Neutrino Beam . . . . . . . . . . . . . . . . 33

3.2 The MINOS Detectors . . . . . . . . . . . . . . . . 35

3.2 .1 The Far Detector . . . . . . . . . . . . . . . . . 40

3.2 .2 The Near Detector . . . . . . . . . . . . . . . . . 42

3.2.3 The Calibration Detector . . . . . . . . . . . . . 45

3.2.4 Detector Co-ordinate systems . . . . . . . . . . . . . 47

3.3 Physics Capabilities . . . . . . . . . . . . . . . . . . . 49

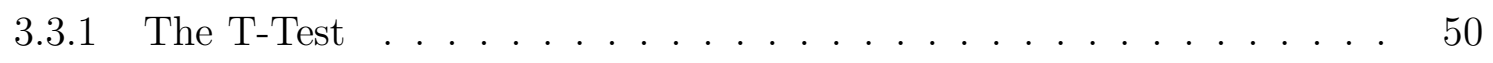


3.3.2 Spectral Dependence Measurements . . . . . . . . . . . . . 51

3.3.3 Appearance Searches . . . . . . . . . . . . . . . . . . 53

4 The MINOS Energy Calibration $\quad 55$

4.1 Energy Calibration Motivation . . . . . . . . . . . . . . . . . 56

4.2 The Light-Injection System Hardware . . . . . . . . . . . . . . . . . . . . . 58

4.3 Muons . . . . . . . . . . . . . . . . . . . . . . . . . 59

4.4 PMT Gain Calibration . . . . . . . . . . . . . . . . . . . . 60

4.5 The Calibration Chain . . . . . . . . . . . . . . . . . . . 62

4.5.1 Drift Calibration ...................... 62

4.5.2 Linearity Calibration . . . . . . . . . . . . . . . 63

4.5.3 Strip-to-Strip Calibration _. . . . . . . . . . . . . . . . . 64

4.5.4 Fibre Attenuation Calibration . . . . . . . . . . . . . . . . 64

4.5.5 Inter-detector Calibration . . . . . . . . . . . . . . . 65

4.5.6 Absolute Energy Calibration . . . . . . . . . . . . . . 67

4.6 Neutrino Energy Calculation . . . . . . . . . . . . . . . . . . . . 68

5 Relative Energy Calibration Technique Using Stopping Muons $\quad 70$

5.1 Muons in MINOS . . . . . . . . . . . . . . . . . . 71

5.1 .1 The Bethe-Bloch Equation . . . . . . . . . . . . . 71

5.1 .2 Muon Energy Loss in the MINOS Detectors . . . . . . . . . . . . 73

5.1 .3 Muon Multiple Scattering . . . . . . . . . . . . . . . . . 75

5.1 .4 Muon Curvature in the Magnetic Field . . . . . . . . . . . . . . 76

5.2 Relative Energy Calibration Technique . . . . . . . . . . . 76

5.2.1 Track Window Technique . . . . . . . . . . . . . . . 77

5.3 Stopping Muon Reconstruction _. . . . . . . . . . . . . . . 81

5.4 The Muon Energy Unit . . . . . . . . . . . . . . . . . . . . . . 85

6 Relative Energy Calibration Results $\quad 89$

6.1 The Calibration Detector . . . . . . . . . . . . . . . . . . . 89

6.1.1 Stopping Muon Selection and Backgrounds . . . . . . . . . . . . . 90 
6.1 .2 Results . . . . . . . . . . . . . . . . . . . . . 100

6.1 .3 Sources of Systematic Error _ . . . . . . . . . . . . . . . 102

6.1.4 A Cross-check against the Test-beam Energy . . . . . . . . . . . . 112

6.1.5 Summary of Errors and Conclusions . . . . . . . . . . . . . . 115

6.2 The Far Detector . . . . . . . . . . . . . . . . . . . . 116

6.2.1 Stopping Muon Reconstruction _. . . . . . . . . . . . 117

6.2.2 Stopping Muon Selection and Backgrounds . . . . . . . . . . 118

6.2 .3 Results . . . . . . . . . . . . . . . . . . . . . . . . . . . 124

6.2 .4 Sources of Systematic Error _ . . . . . . . . . . . . . 125

6.2.5 Summary of Errors and Conclusions . . . . . . . . . . . . . 143

6.3 Measuring the Bethe-Bloch Curve . . . . . . . . . . . . . . . . . . 145

6.3.1 Bethe-Bloch Measurements at the Far Detector . . . . . . . . 145

6.3.2 Bethe-Bloch Measurements at the Calibration Detector . . . . . . . 148

6.4 Performing the Relative Calibration in MC . . . . . . . . . . . . 153

6.4.1 Calibration and "De-calibration" of the MC . . . . . . . . 154

6.4.2 Tuning the MC Detector Simulation "light level" . . . . . . . . . . 154

6.4 .3 Results . . . . . . . . . . . . . . . . . . . 156

6.4.4 Discussion of Results and Conclusion . . . . . . . . . . . . 156

6.5 Summary and Conclusions . . . . . . . . . . . . . . . . . . 159

7 Impact of the Relative Calorimetric Energy Calibration on MINOS 162

7.1 Neutrino Energy Reconstruction in the MINOS Detectors . . . . . . . . . 162

7.2 Impact on Oscillation Parameter Measurement . . . . . . . . . . . . . 164

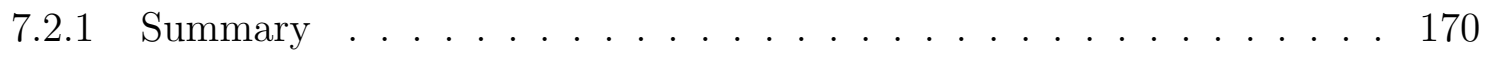

8 Conclusion and Outlook $\quad 173$

8.1 Measurement of the Detectors' Relative Response . . . . . . . . . . . . 174

8.2 The Effect on MINOS of a Relative Miscalibration . . . . . . . . . . . 175

8.3 Outlook for the future $\ldots \ldots \ldots \ldots \ldots \ldots$

$\begin{array}{ll}\text { A Calibration Detector Analysis Details } & 177\end{array}$ 


\section{List of Figures}

2.1 Survival probability of $\nu_{\mu}$ vs. energy for the MINOS baseline. . . . . . . . . 11

2.2 Energy spectra of solar neutrinos f . . . . . . . . . . . . . . . . 15

2.3 Solar neutrino fluxes measured by SNO . . . . . . . . . . . . . . . . . 19

2.4 Prompt energy spectrum of reactor anti-neutrino events observed in KamLAND 20

2.5 Solar neutrino allowed oscillation parameter space . . . . . . . . . . . . . . 22

2.6 A sketch showing the relation between zenith angle and the distance travelled by atmospheric neutrinos . . . . . . . . . . . . . . . . . 25

2.7 Measurements of the atmospheric neutrino flavour ratio of ratios . . . . . . 26

2.8 Zenith angle distributions from Super-Kamiokande . . . . . . . . . . . . . 27

2.9 Super-Kamiokande observed dependence of neutrino flux with $L / E$. . . . . 28

2.10 Super-Kamiokande allowed regions for atmospheric neutrino oscillation parameters. . . . . . . . . . . . . . . . . . . . . . 29

2.11 Summary of current knowledge of neutrino oscillation parameters . . . . . . 32

3.1 Map of neutrino beam used by MINOS . . . . . . . . . . . . . . . . . . 34

3.2 Layout of neutrino beam components . . . . . . . . . . . . . . . . . . . 34

3.3 Magnetic horn configurations and beam energy spectra . . . . . . . . . . 36

3.4 Cross-section of scintillator strip . . . . . . . . . . . . . . . . . . . . . 37

3.5 A short length of MINOS scintillator illuminated by a blue LED. . . . . . . . 38

3.6 Photograph of an open scintillator module . . . . . . . . . . . . . . . . 39

3.7 Photograph of the Far detector . . . . . . . . . . . . . . . . . . . 40

3.8 Photograph of the Near Detector . . . . . . . . . . . . . . . . . . 42

3.9 Sketch of a partially-instrumented Near detector plane . . . . . . . . . . . . 43 
3.10 Four logical sections of the Near Detector . . . . . . . . . . . . . . . . 44

3.11 Photograph of the Calibration detector . . . . . . . . . . . . . . . 46

3.12 MINOS coordinate systems f . . . . . . . . . . . . . . . 48

3.13 Limits on oscillation parameters using the T-test . . . . . . . . . 50

3.14 Parameter determination using $\nu_{\mu}$ CC energy spectra . . . . . . . . . . 51

3.15 Sensitivity of $\nu_{\mu} \rightarrow \nu_{\tau}$ oscillation parameter measurements using spectral dependence measurements . . . . . . . . . . . . . . . . . . . 52

3.16 Limit on $\nu_{\mu} \rightarrow \nu_{e}$ oscillation parameters . . . . . . . . . . . 54

4.1 A schematic diagram of the Light Injection system. . . . . . . . . . . . . 58

5.1 Stopping power for positive muons in copper as a function of momentum . . 72

5.2 Calculated muon energy loss per plane . . . . . . . . . . . . . . . . 74

5.3 Track window position . . . . . . . . . . . . . . . . 78

5.4 Energy deposition in the track window vs. window start position . . . . . . . 80

5.5 Detector response in track window . . . . . . . . . . . . . . 87

6.1 Time-of-flight spectrum from the Calibration detector TOF system . . . . . 91

6.2 Number of planes hit by muons and pions in a GEANT3 MC simulation at

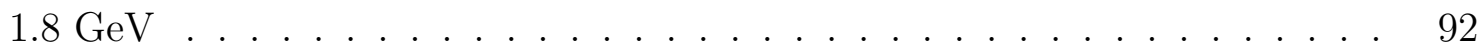

6.3 Number of planes hit by muons and pions between 1.4-2.0 GeV . . . . . . 93

6.4 An overlapping "PS muon" and test-beam muon . . . . . . . . . . . . . 96

6.5 Particle identification cuts at the Calibration detector . . . . . . . . . . 98

6.6 Event selection and background rejection cuts at the Calibration detector . . 99

6.7 Calibration detector response to stopping muons vs. run index . . . . . . . . 101

6.8 Average fractional contribution of individual strips to MEU at $1.8 \mathrm{GeV}$. . 104

6.9 Muon event length at $1.8 \mathrm{GeV}$ in $\mathrm{MC} \ldots \ldots \ldots$. . . . . . . . . 106

6.10 Cross-talk pattern for muons at $1.8 \mathrm{GeV}$ with M16 and M64 PMTs . . . . . 108

6.11 MEU vs. lower track length cut at $1.8 \mathrm{GeV} \ldots \ldots \ldots$. . . . . . . . . . . 109

6.12 Detector regions used in the track window at different energies. . . . . . . 111

6.13 Stopping muon response vs run $(1.4-2.0 \mathrm{GeV} / \mathrm{c}) \ldots \ldots \ldots$. . . . . . . 113 
6.14 Calibrated electron response vs. run index . . . . . . . . . . . . . 114

6.15 Event quality cuts at the Far detector . . . . . . . . . . . . . . 122

6.16 Detector response over time . . . . . . . . . . . . 126

6.17 Detector response at different stages of the calibration . . . . . . . . . . 129

6.18 Detector response after full calibration . . . . . . . . . . . . . . . 130

6.19 Spatial variations in the average detector response . . . . . . . . . . . . 132

6.20 Spatial distribution of strips in track window . . . . . . . . . . . . . 134

6.21 Catastrophic muon energy loss . . . . . . . . . . . . . . . 136

6.22 Detector response centroid vs. truncation threshold . . . . . . . . . . . 137

6.23 Detector response angular dependence and the angular distribution of stopping muons . . . . . . . . . . . . . . . . . . . . . . 139

6.24 Muon energy at the track end . . . . . . . . . . . . . . . . . . 141

6.25 Bethe-Bloch curve for the Far detector . . . . . . . . . . . . . . . . . . 146

6.26 Bethe-Bloch curve for the Far detector after correction . . . . . . . . . . . 147

6.27 Bethe-Bloch curve for the Calibration detector . . . . . . . . . . . . . 149

6.28 Spread in muon track length and its effect on stopping power at the Calibration detector . . . . . . . . . . . . . . . . . . . . 151

6.29 Bethe-Bloch curve for the Calibration detector with restricted muon track

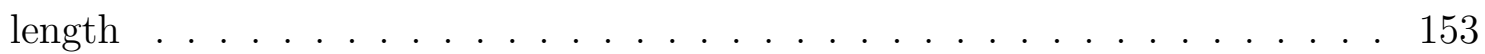

7.1 Neutrino visible energy spectra . . . . . . . . . . . . . . . 164

7.2 Fractional $\Delta m^{2}$ bias vs. calorimetric energy shift . . . . . . . . . . . 168

7.3 Neutrino visible energy spectra for different $\Delta m^{2} \ldots \ldots \ldots \ldots$

7.4 Fractional $\sin ^{2}(2 \theta)$ bias vs. calorimetric energy shift . . . . . . . . . . 171 


\section{List of Tables}

5.1 Contribution of different muon energy loss mechanisms in iron . . . . . . . 73

5.2 Contribution of different muon energy loss mechanisms in polystyrene . . . . 73

5.3 Calculated minimum ionising particle energy loss per plane . . . . . . . . 75

5.4 Muon multiple scattering in steel . . . . . . . . . . . . . . 75

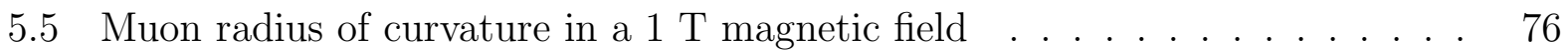

5.6 Error on the MEU value using the median or mean $\ldots \ldots$. . . . . . . 88

6.1 Event length cuts for muons of different energies at the Calibration detector 92

6.2 MEU values for Calibration detector run periods . . . . . . . . . . . . . 102

6.3 Detector regions with readout problems at the Calibration detector . . . . . 103

6.4 Differences in fully calibrated electron response per $\mathrm{GeV}$ in the three Calibration detector run periods . . . . . . . . . . . . . . . . . . 115

6.5 Systematic errors on the Calibration detector MEU value . . . . . . . . . . 116

6.6 Rate of dead chips in planes just beyond track end point at the Far detector 143

6.7 Systematic errors on the Far detector MEU value . . . . . . . . . . . . . . . 144

6.8 Results of relative calibration in $\mathrm{MC} \ldots \ldots \ldots \ldots$

A.1 Calibration detector trigger times and VARC live times . . . . . . . . 177

A.2 Runs used in the Calibration detector MEU calibration . . . . . . . . . 178

A.3 Calibration detector runs not used due to different detector/beam conditions 178 


\section{Chapter 1}

\section{Introduction}

During the last decade, some of the most exciting results in particle physics have come from those experiments studying neutrinos. Although the neutrino was discovered nearly 50 years ago, it is only recently that strong evidence of the three neutrinos' ability to change back and forth between each other has come to light. The ability of neutrinos to oscillate between the three types proves one of the assumptions in the Standard Model of particle physics to be incorrect. Neutrino oscillations in a vacuum mean that at least one of the neutrinos must have a mass, whereas the Standard Model assumes they are all massless.

The focus of new experiments, such as MINOS, is to precisely measure the probability of a neutrino oscillating and the frequency with which it oscillates. In other words, to measure the mixing angle and difference in the squares of the neutrino masses. In order to make a precise measurement of the neutrino oscillation parameters it is necessary to accurately know the energy of the neutrinos used in the experiment and how far they have travelled. The MINOS experiment uses a precisely controlled beam of neutrinos created at the Fermi National Accelerator Laboratory in Illinois. The neutrino beam is measured close to the source with the "Near" detector before it travels $735 \mathrm{~km}$ through the Earth to the Soudan Underground Laboratory in Minnesota where it is measured for a second time with the "Far" detector. A comparison of the neutrino energy spectra from the two detectors should allow MINOS to make the world's most precise measurement of the oscillation parameters 
involved. In addition, it is hoped that the oscillatory signature of neutrino disappearance and reappearance will be observed, thus putting the whole phenomenon of neutrino oscillations on a firmer footing.

In order for MINOS to precisely measure the oscillation parameters it has to be able to reconstruct and compare the energy of neutrino events at the two detectors. Accurate knowledge of both the absolute and the relative energy scale of the detectors is required. As part of the MINOS calibration programme a third, smaller "Calibration" detector was constructed and exposed to particles of a known energy from various test-beams at CERN.

This thesis details a new measurement technique for determining the calorimetric energy scale of the MINOS detectors. The results of such measurements are described and used to perform the relative calibration between the Far detector and the Calibration detector. Measurement of the Near detector's relative calorimetric response will be performed using the techniques described in this thesis once the intra-detector calibration is fully accomplished. The Near detector was the last of the three detectors to be constructed and was only completed in the autumn of 2004 .

Chapter 2 sets out the theory of neutrino oscillations and reviews the results from previous neutrino oscillation experiments. Chapter 3 describes the MINOS detectors and their readout systems before going on to present the anticipated physics measurements that MINOS will make. Chapter 4 sets out the motivation and the targets for the MINOS calorimetric energy calibration. The steps involved in fully calibrating the MINOS detectors are described and the hardware used for some of the calibration steps is outlined.

Chapter 5 describes in detail how stopping muons can be used to perform the relative energy calibration. The physics of stopping muon interactions in the MINOS detectors is outlined. A new "track window" technique for achieving the relative calibration is introduced and described. Details of stopping muon reconstruction and calibration units are given.

Chapter 6 presents the results of a measurement of the relative calorimetric energy scale at the Calibration detector and Far detector. Descriptions of stopping muon selection and 
the potential backgrounds to the sample are given. Detailed studies of the systematic errors are described. The results from measurements of the Bethe-Bloch curve in the two detectors are presented. Details of how the relative calibration was performed in the Monte Carlo (MC) simulation are given.

Chapter 7 describes the results of a study to assess the systematic error on the measurement of the oscillation parameters due to relative calorimetric energy scale miscalibrations. The systematic errors are evaluated for a range of possible oscillation parameter scenarios.

Finally, chapter 8 gives the conclusions of this thesis. The results are summarised and their implication for MINOS is discussed. Ideas for future measurements to further ensure a robust relative calorimetric energy calibration are presented. 


\section{Chapter 2}

\section{Neutrino Physics}

\subsection{The "Little Neutral One" - A Brief History}

It was on December 14th, 1930 in a letter to a radioactivity conference that Wolfgang Pauli first publicly discussed his "wenig wahrscheinlich" (improbable) idea to explain the continuous electron energy spectrum of $\beta$ decay [1]. Pauli's hypothesis was that in radioactive $\beta$ decay a neutron, as he called it, "was emitted along with the electron in such a way that the sum of the energies of the neutron and the electron is constant", which thus saved the principle of the conservation of energy. Pauli postulated that this neutron should be electrically neutral, have a low mass and spin- $1 / 2$ with a small magnetic moment. In 1932 the heavy neutron was discovered by Chadwick and hence later on Pauli's neutron was renamed the neutrino, "the little neutral one", by Enrico Fermi. It was to be another 26 years before the neutrino was experimentally observed but it soon became deeply embedded in the theoretical framework of particle physics.

The now 75 year history of neutrino physics is an extremely interesting one involving many ingenious experiments, several of which deservedly won a Nobel prize. Following the direct detection of the neutrino by Reines et al. in 1956 [2] the next major experimental result was a measurement of the neutrino's helicity. Goldhaber et al. showed that the neutrino was left-handed [3], in agreement with the V-A nature of the weak interaction predicted by 
Feynman and Gell-Mann. A year later, in 1959 Davis et al. showed that the anti-neutrino could be distinguished from the neutrino while at the same time putting an upper limit on the neutrino flux from the sun [4]. Another leap forward took place at the AGS accelerator at Brookhaven just 3 years later. Lederman et al. showed that the neutrinos from $\pi^{ \pm}$decay were different to the neutrinos involved in $\beta$ decay hence discovering the muon neutrino [5].

Throughout the 1960's Davis et al. continued their ambitious project to detect neutrinos created 150 million $\mathrm{km}$ away in the core of the sun but it wasn't until 1971 that they succeeded in measuring the flux [6]. What they found was a disagreement with calculations of the expected flux in the Standard Solar Model: neutrinos were missing and this was the start of the solar neutrino problem that wasn't to be convincingly solved for a further 30 years (see section 2.3.1). In 1973 European researchers had a breakthrough at the Gargamelle experiment at CERN with the discovery of the Neutral Current interaction. This discovery was an important one on the road towards Electroweak unification and the development of the Standard Model of particle physics.

Progress in neutrino physics during the 1980s was to come from an unexpected quarter. Several large water Čerenkov detectors were being used to search for proton decay (see section 2.3.1). The major source of background to these proton decay experiments was from neutrinos created in the atmosphere by high energy cosmic rays. When proton decay wasn't discovered, ironically it was the background that was found to hold the keys to fascinating new physics. The Japanese experiment, Kamiokande, and the American experiment, IMB, reported a deficit in the number of muon neutrinos created in the atmosphere with respect to the number of electron neutrinos. This deficit was to become the second neutrino problem: the atmospheric neutrino problem, which precipitated the MINOS experiment. Coupled with more robust measurements of the solar neutrino deficit, evidence for the neutrino oscillation explanation of the two anomalies was growing. The subject of neutrino oscillations forms the focus of this thesis and is discussed further later in this chapter.

Two other major discoveries were made in the 1980s: firstly, neutrinos from outside our solar system were detected for the first time in the SN 1987A super-nova [7]; secondly, the 
LEP experiment precisely measured the width of the $Z^{0}$-resonance [8]. Measurement of the $Z^{0}$-resonance was highly significant since it proved that there were only 3 light $(<45 \mathrm{GeV})$, active neutrino flavours and hence that all weakly interacting fermions below this mass were accounted for.

In the 1990s the evidence for neutrino oscillations, and consequently non-zero neutrino mass, became much stronger with the results from the Super-Kamiokande experiment (see section 2.3.2). The LSND experiment also presented evidence for neutrino oscillations but this result is treated with caution, both by parties within the LSND collaboration and by the wider neutrino physics community. Confirmation or exclusion of the LSND results is awaited from the MiniBooNE experiment at Fermilab [9, 10].

It wasn't until the turn of the millennium that the DONUT experiment directly detected the tau neutrino by observing the production of taus in a beam of tau neutrinos at Fermilab [11]. Possibly the two most significant experimental results of this current decade are that of the KamLAND and SNO experiments (see section 2.3.1). SNO measured both the total flux of all types of neutrino from the sun and the electron neutrino component of that flux. The total flux agreed extremely well with that predicted from theoretical solar models and knowing the fraction of electron neutrinos allowed significant resolution of the possible neutrino oscillation scenarios. The KamLAND experiment observed an energy dependent anti-neutrino disappearance at the distance predicted by the solar experiments. This independent verification of the "solar" oscillation parameters using a terrestrial source of anti-neutrinos was a significant test of the neutrino oscillation hypothesis.

\section{$2.2 \quad$ Neutrino Mass and Oscillations}

In the standard model of particle physics the neutrinos are assumed to be massless. Direct searches for neutrino mass have only ever yielded upper limits. In the case of the electron neutrino, the upper limit on its mass is many hundreds of thousands of times less than the mass of the electron. Direct kinematic searches $[12,13,14]$ for a non-zero neutrino mass 
have yielded the results

$$
\begin{array}{ll}
m_{\nu_{e}}<2.2 \mathrm{eV} & \left(95 \% \text { C.L., from }{ }^{3} \mathrm{H} \rightarrow{ }^{3} \mathrm{He}+\mathrm{e}^{-}+\bar{\nu}_{\mathrm{e}}\right), \\
m_{\nu_{\mu}}<170 \mathrm{keV} & \left(90 \% \text { C.L., from } \pi^{+} \rightarrow \mu^{+}+\nu_{\mu}\right), \\
m_{\nu_{\tau}}<15.5 \mathrm{MeV} & \left(95 \% \text { C.L., from } \tau \rightarrow 5 \pi+\nu_{\tau}\right) .
\end{array}
$$

However, the existence of vacuum neutrino oscillations proves that at least two of the three neutrinos must have a mass. The value of $\Delta m^{2}$, the difference in the square of two neutrino masses, that is measured in neutrino oscillation experiments puts a lower limit on the mass of the heaviest neutrino $\left(\sqrt{\Delta m^{2}} \simeq 0.05 \mathrm{eV}\right)$.

\subsubsection{Neutrino Oscillations}

Neutrino experiments that determine the neutrino flavour (electron, muon or tau) do so by observing the charged lepton that is produced when the neutrino undergoes a charged current interaction. If the neutrino weak (or flavour) eigenstates are not aligned with the mass eigenstates then mixing and hence oscillations will occur $[15,16]$. The neutrino flavour eigenstates can be written as a linear superposition of the mass eigenstates

$$
\left(\begin{array}{c}
\nu_{e} \\
\nu_{\mu} \\
\nu_{\tau}
\end{array}\right)=\left(\begin{array}{ccc}
U_{e 1} & U_{e 2} & U_{e 3} \\
U_{\mu 1} & U_{\mu 2} & U_{\mu 3} \\
U_{\tau 1} & U_{\tau 2} & U_{\tau 3}
\end{array}\right)\left(\begin{array}{c}
\nu_{1} \\
\nu_{2} \\
\nu_{3}
\end{array}\right)
$$

where $U$ is the Pontecorvo-Maki-Nakagawa-Sakata (PMNS) lepton mixing matrix. The eigenstates propagate according to

$$
\left|\nu_{1}(\mathbf{x})\right\rangle=\left|\nu_{1}(0)\right\rangle e^{-i \mathbf{p}_{1} \cdot \mathbf{x}}
$$


where $\mathbf{p}_{1}$ is the 4 -momentum of state 1 . Thus, a neutrino produced in a pure $\nu_{\mu}$ state evolves as

$$
\left|\nu_{\mu}(\mathbf{x})\right\rangle=U_{\mu 1}\left|\nu_{1}\right\rangle e^{-i \mathbf{p}_{1} \cdot \mathbf{x}}+U_{\mu 2}\left|\nu_{2}\right\rangle e^{-i \mathbf{p}_{2} \cdot \mathbf{x}}+U_{\mu 3}\left|\nu_{3}\right\rangle e^{-i \mathbf{p}_{3} \cdot \mathbf{X}}
$$

Inverting equation 2.1 to give the mass eigenstates as superpositions of the flavour eigenstates and substituting into equation 2.3 yields

$$
\begin{aligned}
\left|\nu_{\mu}(\mathbf{x})\right\rangle & =\left(U_{\mu 1} U_{e 1}^{*} e^{-i \mathbf{p}_{1} \cdot \mathbf{x}}+U_{\mu 2} U_{e 2}^{*} e^{-i \mathbf{p}_{2} \cdot \mathbf{x}}+U_{\mu 3} U_{e 3}^{*} e^{-i \mathbf{p}_{3} \cdot \mathbf{x}}\right)\left|\nu_{e}\right\rangle \\
& +\left(U_{\mu 1} U_{\mu 1}^{*} e^{-i \mathbf{p}_{1} \cdot \mathbf{x}}+U_{\mu 2} U_{\mu 2}^{*} e^{-i \mathbf{p}_{2} \cdot \mathbf{x}}+U_{\mu 3} U_{\mu 3}^{*} e^{-i \mathbf{p}_{3} \cdot \mathbf{x}}\right)\left|\nu_{\mu}\right\rangle \\
& +\left(U_{\mu 1} U_{\tau 1}^{*} e^{-i \mathbf{p}_{1} \cdot \mathbf{x}}+U_{\mu 2} U_{\tau 2}^{*} e^{-i \mathbf{p}_{2} \cdot \mathbf{x}}+U_{\mu 3} U_{\tau 3}^{*} e^{-i \mathbf{p}_{3} \cdot \mathbf{x}}\right)\left|\nu_{\tau}\right\rangle
\end{aligned}
$$

Equation 2.4 shows that for a non-diagonal form of $\mathrm{U}$ then $\nu_{\mu}$ has a contribution from $\nu_{e}$ and $\nu_{\tau}$. The probability of observing $\nu_{\mu}$ at $\mathbf{x}$ is

$$
\mathrm{P}\left(\nu_{\mu} \rightarrow \nu_{\mu}\right)=\left|\left\langle\nu_{\mu} \mid \nu_{\mu}(\mathbf{x})\right\rangle\right|^{2}
$$

Combining equations 2.4 and 2.5, rearranging and applying the unitary relations (e.g. $\left|U_{\mu 1}\right|^{2}+$ $\left.\left|U_{\mu 2}\right|^{2}+\left|U_{\mu 3}\right|^{2}=1\right)$ gives

$$
\begin{aligned}
\mathrm{P}\left(\nu_{\mu} \rightarrow \nu_{\mu}\right)=1 & -4\left|U_{\mu 1}\right|^{2}\left|U_{\mu 2}\right|^{2} \sin ^{2}\left(\frac{\left(\mathbf{p}_{1}-\mathbf{p}_{2}\right) \cdot \mathbf{x}}{2}\right) \\
& -4\left|U_{\mu 1}\right|^{2}\left|U_{\mu 3}\right|^{2} \sin ^{2}\left(\frac{\left(\mathbf{p}_{1}-\mathbf{p}_{3}\right) \cdot \mathbf{x}}{2}\right) \\
& -4\left|U_{\mu 2}\right|^{2}\left|U_{\mu 3}\right|^{2} \sin ^{2}\left(\frac{\left(\mathbf{p}_{2}-\mathbf{p}_{3}\right) \cdot \mathbf{x}}{2}\right) .
\end{aligned}
$$

In the laboratory frame it can be assumed that the neutrino is travelling at approximately the speed of light along the spatial-axis of choice. Thus, $\Delta \mathbf{p} . \mathbf{x}=(\Delta E t-\Delta \bar{p} \cdot \bar{x}) \simeq(\Delta E-\Delta p) x$ where $\Delta \bar{p} \cdot \bar{x}=\Delta p x$ and $\Delta E t \simeq \Delta E x$. Given that the neutrino mass is so small, it is always the case in neutrino oscillation experiments that $E \gg m$, thus the binomial expansion approximation that $p=\left(E^{2}-m^{2}\right)^{1 / 2} \simeq E-m^{2} / 2 E$ can be made. Using the binomial expansion and the approximation that $\mathrm{E}_{1}=\mathrm{E}_{2}=\mathrm{E}$ gives $(\Delta E-\Delta p) x=\Delta m^{2} x / 2 E$, which 
yields

$$
\begin{aligned}
\mathrm{P}\left(\nu_{\mu} \rightarrow \nu_{\mu}\right)=1 & -4\left|U_{\mu 1}\right|^{2}\left|U_{\mu 2}\right|^{2} \sin ^{2}\left(\frac{\Delta m_{12}^{2} L}{4 E}\right) \\
& -4\left|U_{\mu 1}\right|^{2}\left|U_{\mu 3}\right|^{2} \sin ^{2}\left(\frac{\Delta m_{13}^{2} L}{4 E}\right) \\
& -4\left|U_{\mu 2}\right|^{2}\left|U_{\mu 3}\right|^{2} \sin ^{2}\left(\frac{\Delta m_{23}^{2} L}{4 E}\right)
\end{aligned}
$$

where $L$ is the distance travelled by the neutrino when it is observed and $\Delta m_{i j}^{2}=m_{i}^{2}-m_{j}^{2}$. Equations similar to 2.7 may be derived for all neutrino transition probabilities. In general, the transition probability may be written

$$
\mathrm{P}\left(\nu_{\alpha} \rightarrow \nu_{\beta}\right)=\delta_{\alpha \beta}-2 \Re \sum_{j>i} U_{\alpha i} U_{\alpha j}^{*} U_{\beta i}^{*} U_{\beta j}\left(1-e^{\left(i \Delta m_{j i}^{2} L / 4 E\right)}\right),
$$

where $i$ and $j$ represent the mass eigenstates.

The neutrino mixing matrix, $U_{P M N S}$, can be parametrised in an analogous way to the CKM mixing matrix for quarks [17]. Thus,

$$
U_{P M N S}=\left(\begin{array}{ccc}
c_{12} c_{13} & s_{12} c_{13} & s_{13} e^{-i \delta} \\
-s_{12} c_{23}-c_{12} s_{23} s_{13} e^{i \delta} & c_{12} c_{23}-s_{12} s_{23} s_{13} e^{i \delta} & s_{23} c_{13} \\
s_{12} s_{23}-c_{12} c_{23} s_{13} e^{i \delta} & -c_{12} s_{23}-s_{12} c_{23} s_{13} e^{i \delta} & c_{23} c_{13}
\end{array}\right),
$$

where $c_{i j}=\cos \theta_{i j}$ and $s_{i j}=\sin \theta_{i j}(i, j=1,2,3)$. The three angles $\theta_{12}, \theta_{23}$ and $\theta_{13}$ represent the rotations between the mass and flavour eigenstates. The complex phase $\delta$, if non-zero, gives rise to $\mathrm{CP}$ violation in the lepton sector. In considering oscillation experiments, it is convenient to factorise $U_{P M N S}$ into the product of 3 matrices

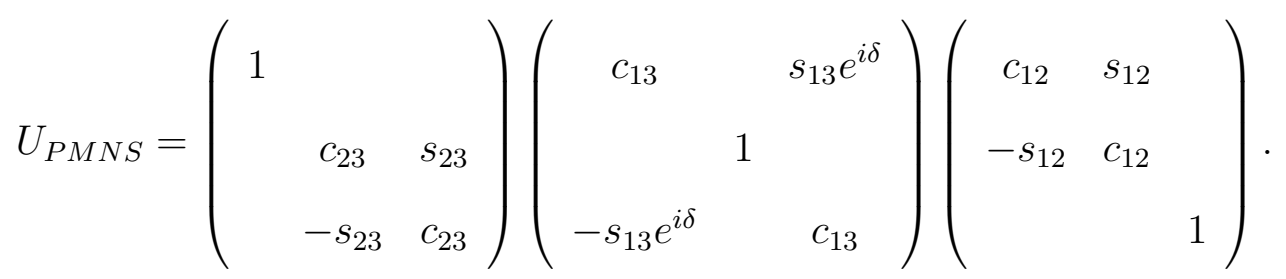

The value of $\theta_{13}$ is known to be small from experiment, thus the second matrix can be approximated as the identity matrix. The first (third) matrix can be seen to only be dependent on $\theta_{23}\left(\theta_{12}\right)$. Given that the values of $\Delta m_{23}^{2}$ and $\Delta m_{12}^{2}$ have been found to differ by a factor of $\sim 25$ the oscillations involving $\theta_{23}$ and $\theta_{12}$ can be considered as de-coupled from an ex- 
perimental viewpoint. As is discussed in later sections, $\theta_{12}$ is associated with oscillations of solar neutrinos and $\theta_{23}$ is associated with oscillations of atmospheric neutrinos.

Given that the oscillation of solar and atmospheric neutrinos is approximately de-coupled it is sensible to consider the more intuitive 2-dimensional case. When only 2 neutrino flavours are considered, the mixing is represented by a single mixing angle $\theta$ with no $\mathrm{CP}$ violating phase. Thus, the flavour eigenstates are related to the mass eigenstates by

$$
\left(\begin{array}{c}
\nu_{\alpha} \\
\nu_{\beta}
\end{array}\right)=\left(\begin{array}{cc}
\cos \theta & \sin \theta \\
-\sin \theta & \cos \theta
\end{array}\right)\left(\begin{array}{c}
\nu_{1} \\
\nu_{2}
\end{array}\right),
$$

where $\nu_{\alpha}$ and $\nu_{\beta}$ represent the two neutrino flavours, and the neutrino mass eigenstates are given by $\nu_{1}$ and $\nu_{2}$. In such a case, the probability for two-flavour mixing is simply

$$
\mathrm{P}\left(\nu_{\alpha} \rightarrow \nu_{\beta}\right)=\sin ^{2}(2 \theta) \sin ^{2}\left(\frac{1.27 \Delta \mathrm{m}^{2} \mathrm{~L}}{\mathrm{E}}\right) \quad(\alpha \neq \beta),
$$

where $\Delta m^{2}$ is expressed in $\mathrm{eV}^{2}$, the detector-to-source distance $L$ in metres $(\mathrm{km})$ and the neutrino energy $E$ in $\mathrm{MeV}(\mathrm{GeV})$. In this form the physical interpretation is straightforward: the mixing probability follows an oscillatory pattern where $\sin ^{2}(2 \theta)$ determines the degree of mixing between the two flavours and $\Delta m^{2} L / E$ dictates the period of the oscillation. An experiment's sensitivity to observing oscillations depends on its choice of $L / E$. In atmospheric neutrino experiments, $L$ and $E$ both vary. In long-baseline accelerator experiments, such as MINOS, the value of $L$ is fixed. Thus, the disappearance of a neutrino flavour varies as a function of energy. Figure 2.1 shows a muon neutrino's survival probability as a function of energy for the MINOS base-line $(L=735 \mathrm{~km})$, with $\Delta m^{2}=2.4 \times 10^{-3}$ and $\sin ^{2}(2 \theta)=0.9$. The MINOS experiment will search for the highest energy dip, which is expected to occur at $\sim 1.5 \mathrm{GeV}$. 


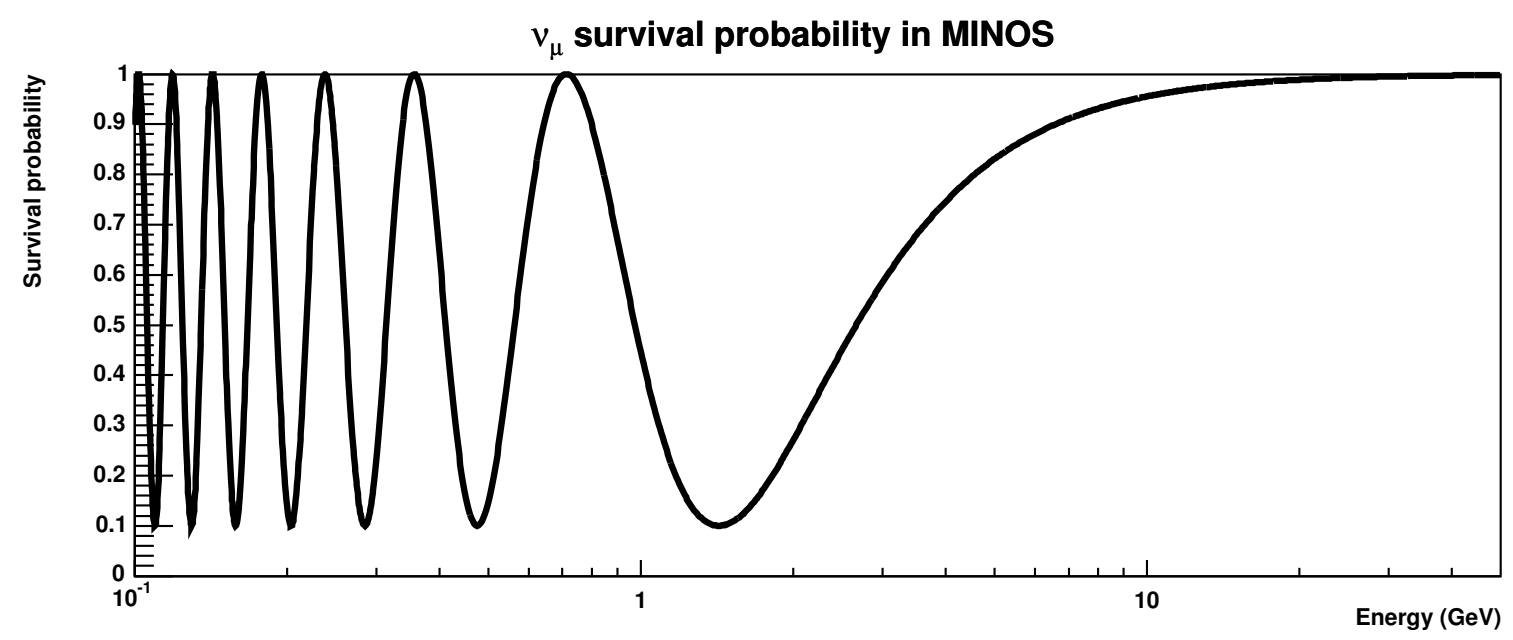

Figure 2.1: Survival probability of $\nu_{\mu}$ vs. energy for the MINOS baseline (figure from [18]). The probability of a $\nu_{\mu}$ surviving after travelling $735 \mathrm{~km}$ is shown as a function of energy for oscillation parameters $\Delta m^{2}=2.4 \times 10^{-3}$ and $\sin ^{2}(2 \theta)=0.9$. The first oscillation dip occurs at $\sim 1.5 \mathrm{GeV}$. The dip does not extend all the way to the $\mathrm{x}$-axis since the mixing is not quite maximal.

\section{Sub-dominant Oscillation Modes}

In recent years much focus of experimental neutrino physics has shifted to determining if the value of $\theta_{13}$ is non-zero and, if so, measuring that value. The smallness of $\theta_{13}$ (relative to $\theta_{12}$ and $\theta_{23}$ ) means that the oscillations that occur are relatively small in magnitude and hence are referred to as being sub-dominant. Two particular avenues of investigation into the value of $\theta_{13}$ are, firstly, looking for electron neutrino disappearance and, secondly, looking for electron neutrino appearance in a source of muon neutrinos. Consider first the case of electron neutrino disappearance. The survival probability of an electron neutrino is to leading order given by

$$
\mathrm{P}\left(\nu_{e} \rightarrow \nu_{e}\right) \simeq 1-\sin ^{2}\left(2 \theta_{13}\right) \sin ^{2}\left(\frac{1.27 \Delta m_{13}^{2} L}{E}\right)-\cos ^{4}\left(\theta_{13}\right) \sin ^{2}\left(2 \theta_{12}\right) \sin ^{2}\left(\frac{1.27 \Delta m_{12}^{2} L}{E}\right)
$$

Given the factor of $\sim 25$ between $\Delta m_{13}^{2}$ and $\Delta m_{12}^{2}$ oscillations involving the first term occur at much lower values of $L / E$. Once the value of $L / E$ approaches $1 / \Delta m_{12}^{2}$ then the oscillations involving the second sinusoidal term dominate with the oscillations of the first sinusoidal term appearing as a small modulation or "ripple". 
Consider now the case of electron neutrino appearance in a source of muon neutrinos. The probability of electron neutrino appearance is to leading order (assuming $\theta_{13}$ is not too small) given by

$$
\mathrm{P}\left(\nu_{\mu} \rightarrow \nu_{e}\right) \simeq \sin ^{2}\left(\theta_{23}\right) \sin ^{2}\left(2 \theta_{13}\right) \sin ^{2}\left(\frac{1.27 \Delta m_{23}^{2} L}{E}\right)
$$

Thus it can be seen that the peak probability of observing $\nu_{e}$ appearance occurs at $L / E \simeq$ $1 / \Delta m_{23}^{2}$ and that the measurement of $\theta_{13}$ is dependent on the value of $\theta_{23}$.

As can be easily seen from the parameterisation of the PMNS matrix given in equation 2.10 the possibility of observing $\mathrm{CP}$ violation in the neutrino sector requires the value of $\theta_{13}$ to be non-zero. If the value of $\theta_{13}$ is zero then the off-diagonal elements of the second matrix will be zero. The complex phase, $\delta$, which potentially gives rise to $\mathrm{CP}$ violation enters the oscillation probability $\mathrm{P}\left(\nu_{\mu} \rightarrow \nu_{e}\right)$ as a sub-leading term, see [19] for details.

\subsubsection{Matter Effects}

So far, the discussion of neutrino oscillations has been about vacuum oscillations but the effects of matter can be significant. When neutrinos travel through the matter making up, say, the Sun or the Earth they undergo coherent elastic forward scattering. The amplitude for such scattering is enhanced for electron neutrinos because they can undergo chargedcurrent as well as neutral-current interactions with the electrons making up the matter. In contrast, muon and tau neutrinos can only undergo neutral-current interactions. The effect of this coherent forward scattering is to endow the electron neutrino with an "effective" mass relative to the muon and tau neutrinos.

The effect on neutrino oscillations of the neutrinos interaction with matter is dubbed the MSW effect after Wolfenstein who first proposed the concept [20] and Mikheev and Smirnov who expanded his ideas [21]. The effect of the medium on the neutrinos traversing it is described by an effective potential that depends on the composition and density of matter. For $\nu_{e}$ the potential is given by $V=\sqrt{2} G_{F} N_{e}$, where $G_{F}$ is the Fermi coupling constant and 
$N_{e}$ is the electron density in the medium. This potential gives rise to an effective mixing and mass matrix. The MSW effect has a significant impact on the oscillations of solar neutrinos due to the high electron density in the sun. For oscillations of atmospheric neutrinos the consequences of a matter effect depends on whether the oscillation mode is $\nu_{\mu} \rightarrow \nu_{\tau}$ or $\nu_{\mu} \rightarrow \nu_{s}$, where $\nu_{s}$ is a sterile neutrino (see section 2.3.3). The signature for matter effects in the sterile case is a suppression of the oscillation probability and a higher mass squared splitting that increases with the energy of the neutrinos.

\subsection{Experimental Evidence for Neutrino Oscillations}

The evidence for neutrino oscillations has grown remarkably over the last decade. Although there are still some predictions of the theory remaining to be convincingly demonstrated, neutrino oscillations are generally accepted as the solution to the solar and atmospheric neutrino anomalies.

\subsubsection{Solar Neutrinos}

It is the discrepancy between the theoretical predictions of the solar neutrino flux and what is experimentally measured that was dubbed the solar neutrino problem. After 30 years of research it is now accepted that new physics is required to explain the anomaly. Thus, it is the study of neutrinos produced in the sun that has provided much of the evidence for neutrino oscillations. The original solar neutrino experiment used a chlorine based radio-chemical technique but since then large water Čerenkov detectors have been used. In addition, further radio-chemical experiments using gallium have been performed.

\section{Standard Solar Model}

Before the first large experiments to try and detector solar neutrinos were undertaken calculations of the expected flux of neutrinos were made. These calculations were the start of 
what is now known as the Standard Solar Model (SSM). The most widely used SSM in solar neutrino physics is that by Bahcall and collaborators (for example BP2004 [22]).

The sun produces its energy by fusing together light nuclei. The chain of thermonuclear reactions that produce the vast majority of the energy in our sun is known as the p-p chain. The end result of this chain is the reaction:

$$
4 p+2 e^{-} \rightarrow{ }^{4} \mathrm{He}+2 \nu_{\mathrm{e}}+26.73 \mathrm{MeV} .
$$

In addition, neutrinos are also emitted by electron capture on ${ }^{7} \mathrm{Be}$ and $\beta$ decay of the elements ${ }^{8} \mathrm{~B},{ }^{13} \mathrm{~N},{ }^{15} \mathrm{O}$ and ${ }^{17} \mathrm{~F}$. These elements are created by solar fusion after the initial pp chain. The energy spectra of the solar neutrinos created by the various different processes are shown in Figure 2.2. It can be seen that the flux of p-p neutrinos dominates with $91 \%$ of the total and that the ${ }^{7} \mathrm{Be}$ contributes a further $7 \%$ of the total. The flux of ${ }^{8} \mathrm{~B}$ neutrinos is only $\sim 0.01 \%$ of the total but their higher energy makes detecting them experimentally advantageous.

Much effort has gone into testing and improving the SSM over the last 30-40 years. Initially there was scepticism about the accuracy of the predictions of the SSM. However, in the mid-1990s satellite measurements of the speed of sound in the sun (helioseismology) verified many of the model's predications. This led to further acceptance of the SSM in the neutrino physics community.

\section{Radio-chemical Experiments}

The first measurement of the solar neutrino flux was made by the Homestake Chlorine experiment [6] in the early 1970s. The experiment measures the rate of ${ }^{37}$ Ar production due to electron neutrino capture on ${ }^{37} \mathrm{Cl}$. The reaction has a threshold of $0.814 \mathrm{MeV}$ and is given by:

$$
\nu_{e}+{ }^{37} \mathrm{Cl} \rightarrow{ }^{37} \mathrm{Ar}+\mathrm{e}^{-} .
$$




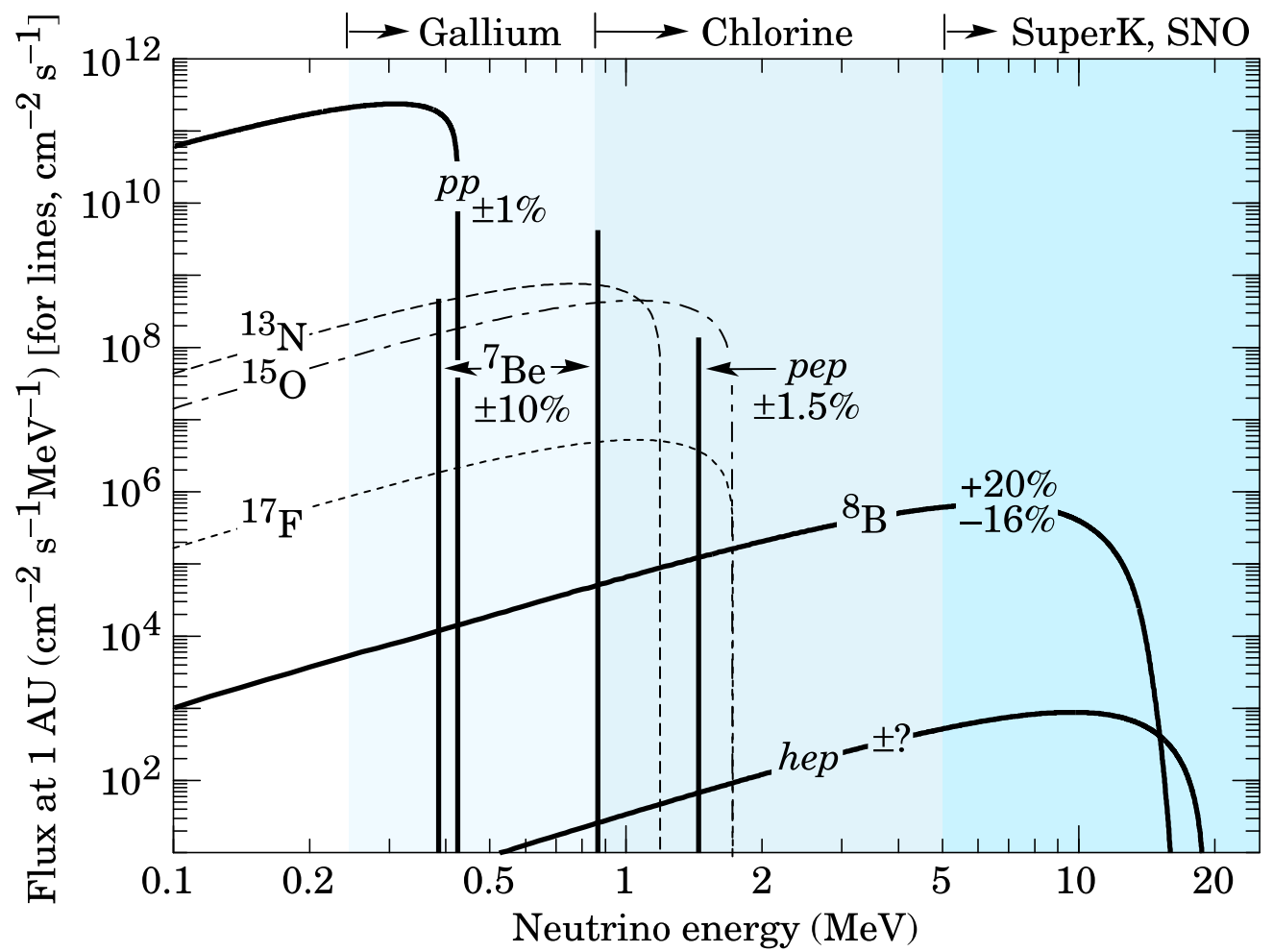

Figure 2.2: Energy spectra of solar neutrinos (figure from [23]). The spectrum of neutrinos emitted in the $\mathrm{p}$-p fusion chain, the electron capture on ${ }^{7} \mathrm{Be}$ and the $\beta$ decay of ${ }^{8} \mathrm{~B},{ }^{13} \mathrm{~N},{ }^{15} \mathrm{O}$ and ${ }^{17} \mathrm{~F}$ are shown with their associated uncertainties. The different colour regions represent the range of energies that the different solar neutrino experiments are sensitive to. 
The chlorine is provided in the form of 615 tons of liquid tetrachloroethylene $\left(\mathrm{C}_{2} \mathrm{Cl}_{4}\right)$. To shield the experiment from cosmic muons it was conducted $1478 \mathrm{~m}$ underground in the Homestake Gold Mine. Extraction of the ${ }^{37} \mathrm{Ar}$ is achieved by periodically flushing the tetrachloroethylene with helium, followed by passing the helium through a charcoal trap to separate the ${ }^{37} \mathrm{Ar}$. The detection of the ${ }^{37} \mathrm{Ar}$ is performed using proportional counters to measure the $2.8 \mathrm{keV}$ Auger electrons emitted when the ${ }^{37} \mathrm{Ar}$ undergoes K-capture.

The measured flux of solar neutrinos by the Homestake Chlorine experiment is $2.56 \pm$ 0.16 (stat.) \pm 0.16 (syst.) Solar Neutrino Units (SNU), where $1 \mathrm{SNU}=1 \times 10^{-36}$ neutrino interactions per target atom per second. The expected flux calculated using the SSM [22] is $8.5 \pm 1.8$ SNU. Thus, the measured flux is only 0.30 of that expected.

Beginning in the late 1980s further radio-chemical experiments were done using gallium. The SAGE [24], GALLEX [25] and later GNO [26] experiments exploited the reaction:

$$
\nu_{e}+{ }^{71} \mathrm{Ga} \rightarrow{ }^{71} \mathrm{Ge}+\mathrm{e}^{-} .
$$

The advantage of using gallium instead of Chlorine is that the threshold for the interaction is only $0.224 \mathrm{MeV}$. This means that the experiment is sensitive to the dominant p-p solar neutrino flux. Consequently the gallium experiments are much smaller, using only 30-60 tons of either liquid gallium (SAGE) or gallium in a hydrochloric solution (GALLEX, GNO). The germanium is chemically extracted and then counted in an analogous fashion to the argon produced in the chlorine experiment.

The measured flux of solar neutrinos by the gallium experiments is significantly higher than that measured by the chlorine experiment because of the sensitivity to the p-p neutrinos. A flux of $70.8 \pm 4.5$ (stat.) \pm 3.8 (syst.) SNU is determined from the combined GALLEX+GNO results [27], the SAGE results are very similar. The expected flux from the SSM [22] is $131_{-10}^{+12}$ SNU. Thus, the measured flux is only 0.54 of that expected. 


\section{Water Čerenkov Experiments}

Some of the most revealing measurements of solar neutrinos have been made by water Čerenkov experiments. The Kamiokande [28], Super-Kamiokande (SK) [29] and SNO [30] experiments detect neutrino events in real-time by detecting the Čerenkov light from electrons recoiling as a consequence of neutrino interactions. In addition, phase III of the SNO experiment uses an array of ${ }^{3} \mathrm{He}$ proportional counters for neutron detection.

Kamiokande and its successor SK are cylindrical detectors containing 680 tons and 22500 tons of water in their fiducial volumes respectively. Located $1000 \mathrm{~m}$ underground, these experiments measure the recoiling electron from elastic scattering (ES) of neutrinos:

$$
\nu_{x}+e^{-} \rightarrow \nu_{x}+e^{-}
$$

where $x=e, \mu, \tau$. Although sensitive to all 3 neutrino flavours the interaction of $\nu_{e}$ dominates since it can undergo $\mathrm{CC}$ as well as $\mathrm{NC}$ interactions. The threshold for detecting the recoiling electron is $7 \mathrm{MeV}$ and $5 \mathrm{MeV}$ in Kamiokande and $\mathrm{SK}$ respectively. Consequently the experiments are only sensitive to the neutrinos from solar ${ }^{8} \mathrm{~B}$ decay plus a tiny contribution from the hep fusion reaction. The recoil of the electron is strongly correlated to initial neutrino direction, which aids background rejection enormously. Furthermore, the number of hit photomultiplier tubes (PMTs) is a measure of the electron energy.

The measured flux of solar neutrinos by SK [31] is $2.32 \pm 0.03$ (stat.) ${ }_{-0.07}^{+0.08}$ (syst.) $\times$ $10^{+6} \mathrm{~cm}^{-2} \mathrm{~s}^{-1}$. The SSM [22] prediction is $5.79(1 \pm 0.23) \times 10^{+6} \mathrm{~cm}^{-2} \mathrm{~s}^{-1}$. Thus, the measured flux is only 0.40 of that expected. The energy spectrum is found to be very similar to that expected from ${ }^{8} \mathrm{~B}$ decay and no significant distortion due to the MSW effect has been observed.

The SNO experiment uses 1000 tons of heavy water and is located deep underground (2092 m). SNO detects solar neutrinos through the charged-current (CC) and neutral- 
current (NC) interactions on the deuteron, and by elastic scattering on electrons:

$$
\begin{aligned}
& \nu_{e}+d \rightarrow e^{-}+p+p-1.442 \mathrm{MeV}(\mathrm{CC}) \\
& \nu_{x}+d \rightarrow \nu_{x}+p+n-2.224 \mathrm{MeV}(\mathrm{NC}) \\
& \nu_{x}+e^{-} \rightarrow \nu_{x}+e^{-}(\mathrm{ES})
\end{aligned}
$$

where $x=e, \mu, \tau$, or any other active neutrino flavour. The neutron produced in the NC interaction is detected via different means in the 3 phases of the experiment. In phase I and II the neutrons are detected via the Čerenkov light from the $\gamma$-rays emitted as a consequence of neutron capture (on deuteron in phase I and chlorine in phase II). In phase III the neutrons are detected using an array of ${ }^{3}$ He proportional counters. The measured fluxes [30] for each reaction (in units of $10^{6} \mathrm{~cm}^{2} \mathrm{~s}^{-1}$ ) are:

$$
\begin{aligned}
& \left.\phi_{C C}=1.68_{-0.06}^{+0.06}(\text { stat. })_{-0.09}^{+0.08} \text { (syst. }\right) \\
& \left.\phi_{E S}=2.35_{-0.22}^{+0.22} \text { (stat. }\right)_{-0.15}^{+0.15} \text { (syst.) } \\
& \left.\phi_{N C}=4.94_{-0.21}^{+0.21} \text { (stat. }\right)_{-0.34}^{+0.38} \text { (syst.) }
\end{aligned}
$$

The advantage of using heavy water over light water is that the flux of electron type neutrinos can be separated from the total flux of active neutrinos. Figure 2.3 shows the flux of $\mu+\tau$ neutrinos vs the flux of electron neutrinos. The solid bands show the allowed fluxes determined by the CC, NC and ES measurements. The intersection of the 3 bands allows resolution of the fluxes of the $\mu+\tau$ neutrinos and shows the consistency of the 3 measurements.

\section{Scintillator Detectors}

Although not yet strictly a solar neutrino experiment ${ }^{1}$, KamLAND [33] probes the same vacuum oscillation parameters, assuming $\mathrm{CPT}$ invariance, as the radio-chemical and water

\footnotetext{
${ }^{1}$ KamLAND aims to make a measurement of the ${ }^{7}$ Be solar neutrino flux in the future but first has to reduce the background from radioactivity in the detector.
} 


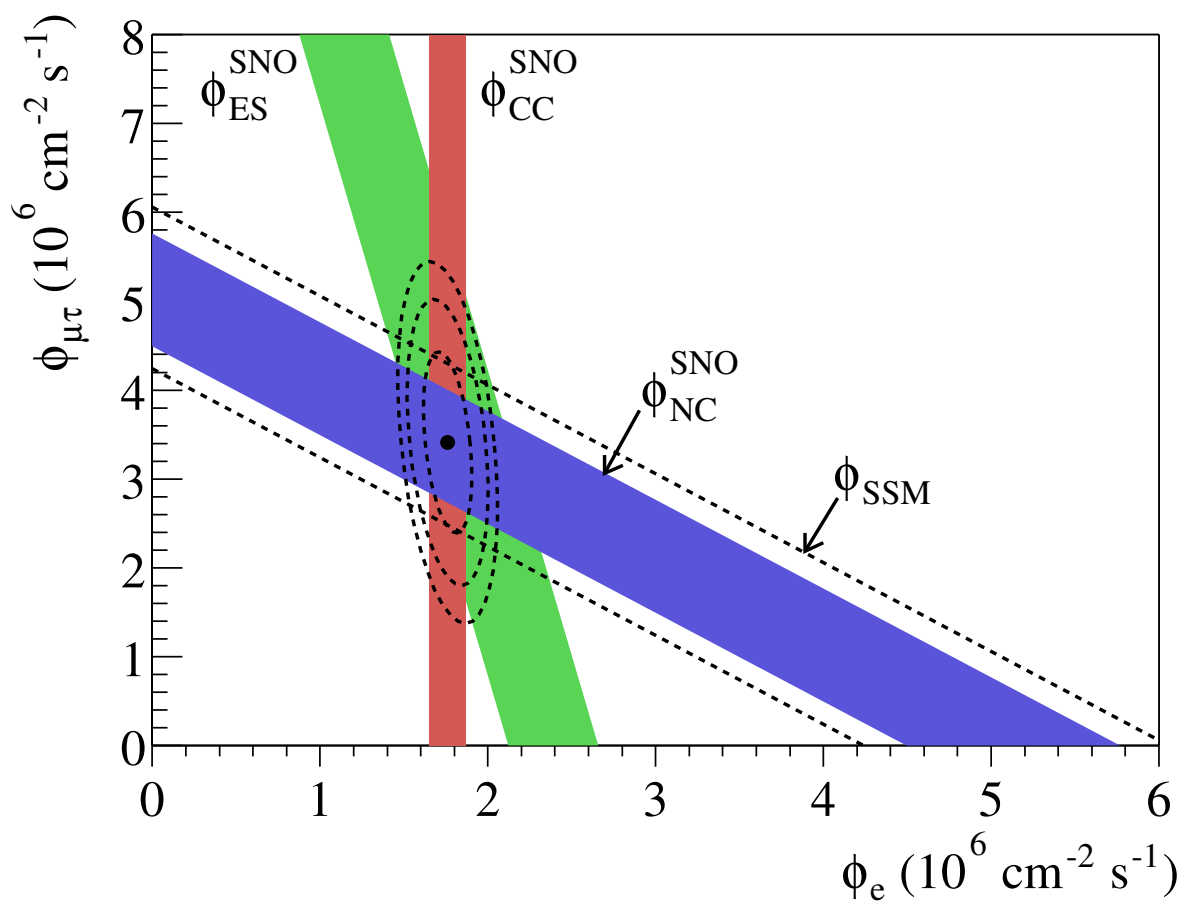

Figure 2.3: Solar neutrino fluxes measured by SNO (figure from [32]). The flux of $\mu+\tau$ neutrinos vs the flux of electron neutrinos is shown. The solid bands show the CC, NC and ES (SNO and SK) flux measurements. The dashed line shows the SSM model prediction. The best fit point is shown with $68 \%, 95 \%$ and $99 \%$ contours.

Čerenkov experiments. Situated at between 100-1000 km from Japan's nuclear reactors, KamLAND detects the $\bar{\nu}_{e}$ emitted from the decay of the neutron-rich fission fragments in the reactors. The majority of the $\bar{\nu}_{e}$ flux at the detector site is from reactors located at approximately $180 \mathrm{~km}$, thus giving better resolution in $L / E$.

The KamLAND detector is located in the same underground cavern that used to house the Kamiokande experiment. An inner detector (ID) holds 1000 tons of ultra-pure liquid scintillator. Surrounding the ID is the outer detector containing 3200 tons of water to act as a shield against radioactivity in the rock as well as a veto for cosmic muons. KamLAND detects $\bar{\nu}_{e}$ via the inverse $\beta$-decay reaction:

$$
\bar{\nu}_{e}+p \rightarrow e^{+}+n
$$

The rapid annihilation of the $e^{+}$followed by the $2.2 \mathrm{MeV} \gamma$-ray from neutron capture on a proton in delayed coincidence is a powerful tool for reducing backgrounds. The incoming 


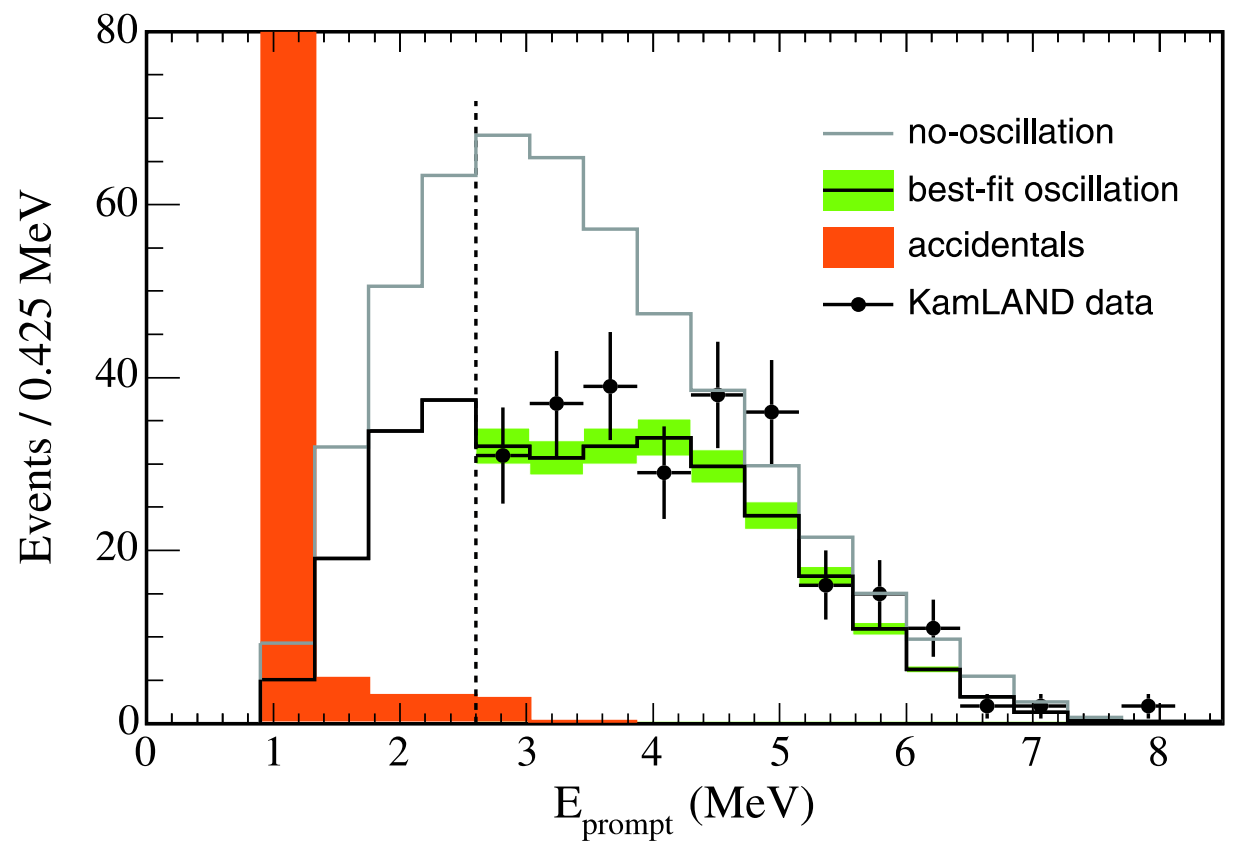

Figure 2.4: Prompt energy spectrum of reactor anti-neutrino events observed in KamLAND (figure from [34]). The measured and expected prompt energy spectra of anti-neutrino candidate events, along with the best-fit oscillation scenario are shown. The dashed line shows the position of the $2.6 \mathrm{MeV}$ analysis threshold.

neutrino energy is estimated using the prompt scintillation light produced by the $e^{+}$. The energy of the $\bar{\nu}_{e}$ is related to the prompt energy $\left(E_{\text {prompt }}=\right.$ kinetic + annihilation energy $)$ and the average neutron recoil energy by $E_{\bar{\nu}_{e}}=E_{\text {prompt }}+\bar{E}_{n}+0.8 \mathrm{MeV}$.

The measured flux of reactor anti-neutrinos [34] is $0.658 \pm 0.044$ (stat.) \pm 0.047 (syst.) of the expected value. Moreover, strong evidence for spectral distortion of the measured $\bar{\nu}_{e}$ has been reported [34]. Figure 2.4 shows the prompt energy spectrum from the observed reactor anti-neutrinos, where the energy dependent disappearance of the anti-neutrinos is clear.

\section{Neutrino Oscillations as the solution of the Solar Neutrino Problem}

As described above, the early solar neutrino experiments observed a deficit in the number of neutrinos arriving from the sun. There were a number of plausible explanations, for example: an erroneous SSM, neutrino decay/decoherence, the existence of a neutrino magnetic moment, or neutrino oscillations. Neutrino quantum decoherence is a generalised term used to explain neutrino flavour change due to coherence loss of the neutrinos' quantum mechan- 
ical phases. This process can occur in the standard model due to the mass eigenstates of the neutrinos travelling at different velocities and hence separating over large distances (for example neutrinos from supernovae are a possibility). However, various mechanisms beyond the Standard Model are also considered that could explain the observed neutrino deficits in the atmospheric and solar case. The mechanism that is commonly considered is related to quantum gravity [35]. Microscopic black holes forming for short periods of time can cause neutrinos to lose quantum information and hence undergo flavour change.

Until the SNO experiment, no solar-model independent evidence of solar neutrino oscillation was observed. However, the parameter space in which oscillations could exist was significantly constrained by the pre-SNO experiments. For example: the variations in the neutrino flux as a function of the Earth's distance from the sun showed no variation other than the expected $1 / r^{2}$ dependence; and there was no observable energy dependent distortion in the ratio of the measured ${ }^{8} \mathrm{~B}$ flux to the SSM calculated flux.

The power of the SNO experiment is in measuring the total flux of all flavours of active neutrino as well as the electron neutrino contribution. The presence of neutrino flavours other than just electron neutrinos violates the hypothesis test for no flavour change at $>7 \sigma$. SNO's measurements also allow determination of the oscillation parameters independently of the solar model flux predictions.

The relatively short baseline of the KamLAND experiment and its near independence of the MSW effect has enabled it to significantly constrain the value of $\Delta m^{2}$. In addition, the observed spectral distortion gives yet more evidence to support neutrino oscillations as the cause of the observed deficit.

A global analysis [30] of all the solar neutrino experiments plus KamLAND gives the result:

$$
\begin{aligned}
\Delta m_{\text {solar }}^{2} & =8.0_{-0.4}^{+0.6} \times 10^{-5} \mathrm{eV}^{2} \\
\theta_{\text {solar }} & =33.9_{-2.2}^{+2.4} \text { degrees }
\end{aligned}
$$




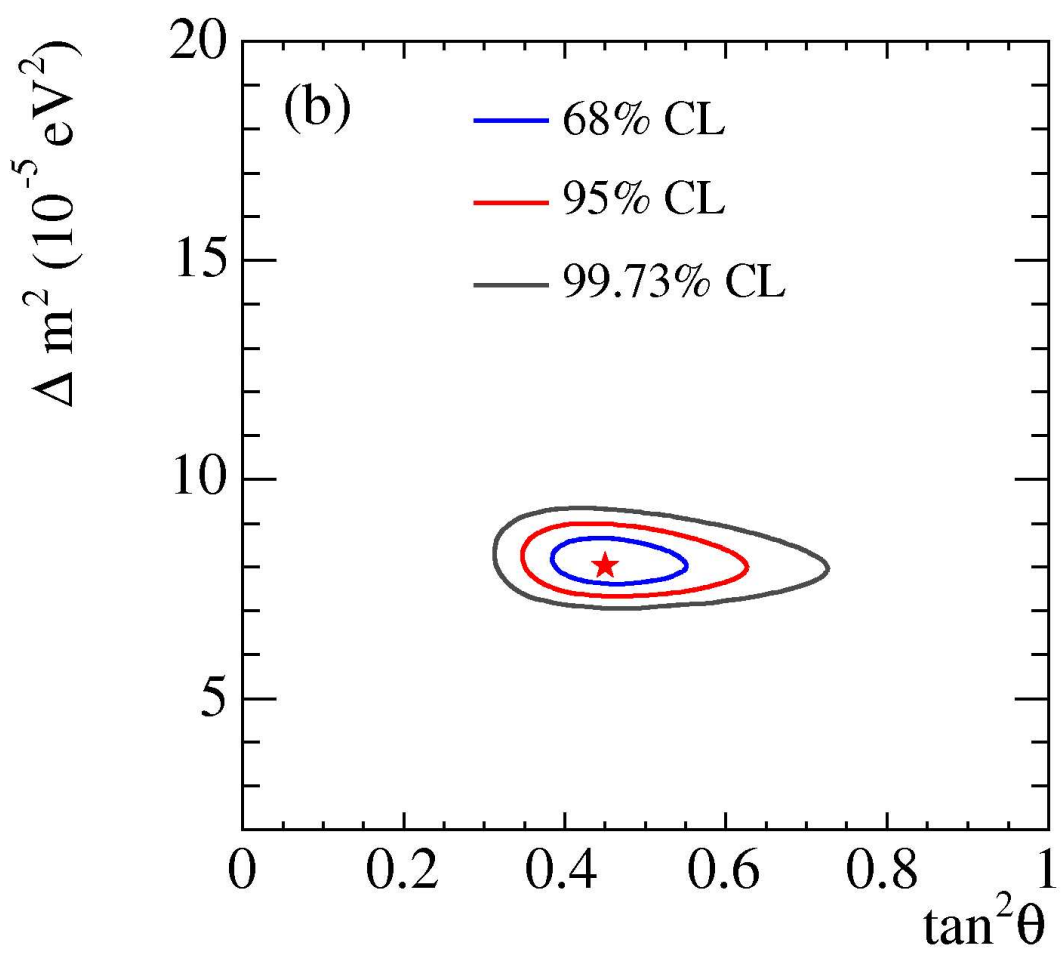

Figure 2.5: Solar neutrino allowed oscillation parameter space (figure from [30]). The 68\%, $95 \%$ and $99.73 \%$ contours from the global analysis presented in [30] are shown.

Figure 2.5 shows the contours in the 2 -dimensional oscillation parameter space. It is interesting to note that after 37 years of experiments it is only in the last couple of years that plots on a non-log scale have been made.

\subsubsection{Atmospheric Neutrinos}

The discovery of the atmospheric neutrino problem happened primarily as a consequence of the search for proton decay: the neutrinos produced in the atmosphere are a significant background to potential proton decays. The proton decay experiments discovered a discrepancy between the flux of muon neutrinos produced in the atmosphere directly above the experiment and those produced on the other side of the Earth. In contrast, the flux of atmospheric electron neutrinos showed no such discrepancy. A number of different water Čerenkov, iron calorimeter and scintillator experiments have been used to study atmospheric neutrinos. 


\section{Atmospheric Neutrino Flux Calculations}

Atmospheric neutrinos are created when cosmic rays strike the Earth's atmosphere. The primary cosmic ray flux is dominated by $\mathrm{H}$ (95\%), with a smaller contribution from $\mathrm{He}$ (4.5\%) above $2 \mathrm{GeV} /$ nucleon. The remaining flux $(0.5 \%)$ is from $\mathrm{C}, \mathrm{N}$ and $\mathrm{O}$ plus heavier nuclei. However, He and heavier nuclei carry approximately $20 \%$ of the nucleons and thus contribute $\sim 20 \%$ to the neutrino flux. The neutrinos are produced mainly by the following reactions:

$$
\begin{aligned}
p+N & \rightarrow n \pi^{ \pm}+X, \\
\pi^{ \pm} & \rightarrow \mu^{ \pm}+\nu_{\mu}\left(\bar{\nu}_{\mu}\right) \\
\mu^{ \pm} & \rightarrow e^{ \pm}+\nu_{e}\left(\bar{\nu}_{e}\right)+\bar{\nu}_{\mu}\left(\nu_{\mu}\right)
\end{aligned}
$$

For secondaries $<\sim 2 \mathrm{GeV}$ the ratio

$$
R=\frac{N\left(\nu_{\mu}+\bar{\nu}_{\mu}\right)}{N\left(\nu_{e}+\bar{\nu}_{e}\right)}
$$

is expected to be $\sim 2$. Higher energy secondaries, in particular muons, are likely to strike the Earth's surface before they decay thus R increases with energy.

In performing calculations of the expected atmospheric neutrino flux there are many factors to consider $[36,37]$. The experimentally determined energy spectra and composition of the primary cosmic rays are used as a starting point. The primaries are propagated in 3-dimensions through a model of the Earth's geo-magnetic field, which produces significant variations in the neutrino flux depending on longitude and latitude. A model of the density structure of the atmosphere is used to determine both where and in what quantity the primaries interact. Uncertainties in the production of secondaries, the charged-pion multiplicities and the pion momentum spectrum introduce errors to the neutrino flux. Thus, the absolute flux of atmospheric neutrinos is only accurate to $\sim 20 \%$. However, because the fluxes of $\nu_{\mu}$ and $\nu_{e}$ are correlated the error in $\mathrm{R}$ is only $\sim 5 \%$ over the broad energy range 
$0.1 \rightarrow 10 \mathrm{GeV}$. When experimental data are presented the ratio of ratios

$$
R^{\prime}=\frac{R_{D A T A}}{R_{M C}}
$$

is typically used, where $R_{D A T A}$ is the value of equation 2.20 from data and $R_{M C}$ is that from MC simulations. It is the deviation of $R^{\prime}$ from unity that was dubbed the atmospheric neutrino problem.

The wide range of energies and baselines over which atmospheric neutrinos are produced enables them to be used to look for neutrino oscillations over a wide range of the $\Delta m^{2}-$ $\sin ^{2}(2 \theta)$ parameter space. The neutrinos produced in the atmosphere above a detector have typically travelled only $\sim 20 \mathrm{~km}$. In contrast, those coming up from the other side of the Earth have travelled nearly $12000 \mathrm{~km}$. Atmospheric neutrino experiments can effectively show their data as a function of distance travelled by using the zenith angle (as shown in Figure 2.6). To first order, the flux of neutrinos as a function of zenith angle is expected to be constant, in the absence of neutrino oscillations.

\section{Early Atmospheric Neutrino Experiments}

The first atmospheric neutrinos were detected in the 1960s. Experiments located extremely deep underground ( $~ 8000$ mwe) at the Kolar Gold Field in South India [39] and at the East Rand Proprietary mine in South Africa [40, 41] reported the observation of atmospheric neutrino interactions in the rock surrounding the detectors. The first fully contained atmospheric neutrino interactions, in which all the visible secondary particles stop in the detector, were detected by proton decay experiments in the early 1980s.

In 1986, the IMB experiment reported a deficit in the number of events in which there was a muon decay as a fraction of the total number of neutrino interactions [42]. The IMB experiment was a large $3.3 \mathrm{kT}$ ring-imaging water Čerenkov experiment and they observed only $26 \% \pm 3 \%$ of their neutrino events had an identified muon decay, whereas they expected to see $34 \% \pm 1 \%$. However, they did not rule out the possibility of a systematic error 


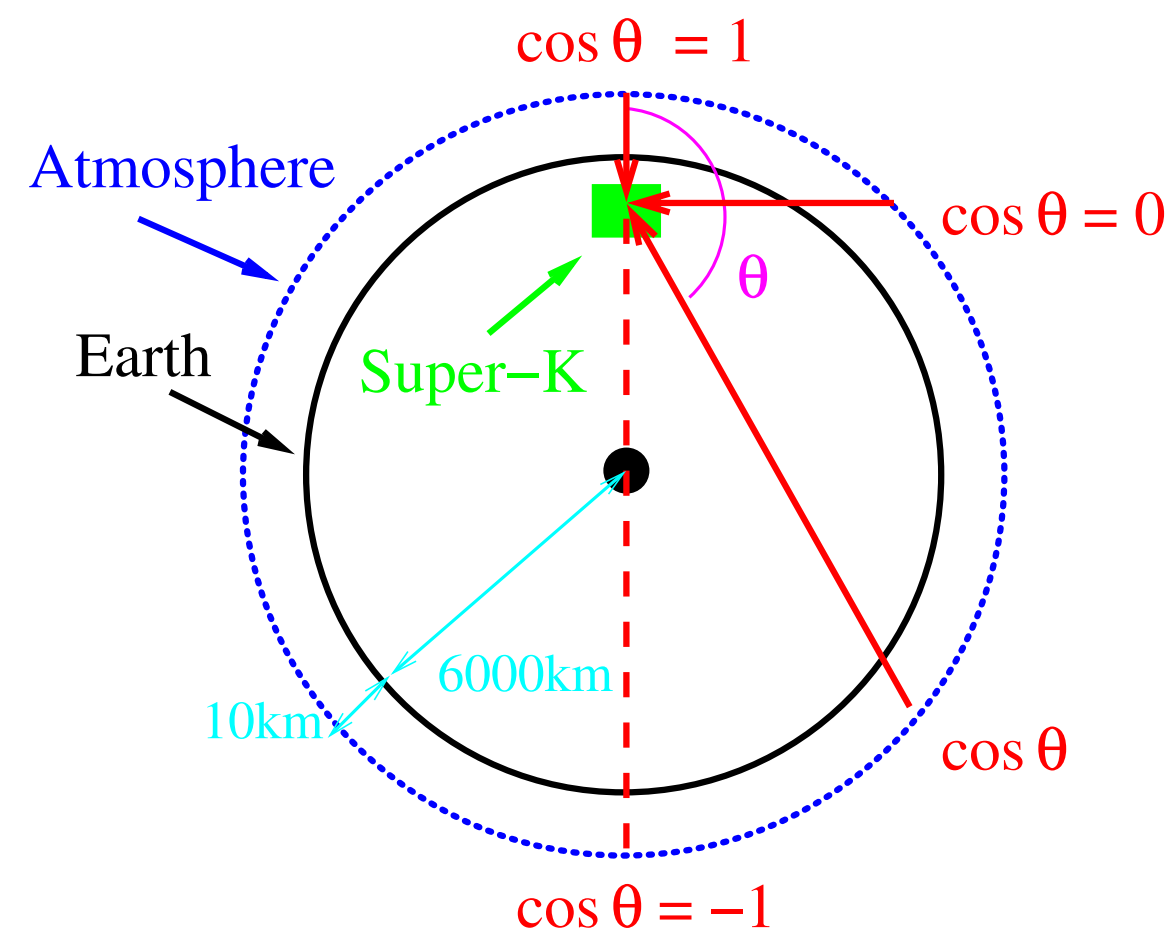

Figure 2.6: A sketch showing the relation between zenith angle and the distance travelled by atmospheric neutrinos (figure from [38]). The wide range of baselines $(\sim 20-12000 \mathrm{~km})$ makes tests of neutrino oscillations using atmospheric neutrinos sensitive to a large region of the $\Delta m^{2}-\sin ^{2}(2 \theta)$ parameter space.

in calculations of $\nu$ fluxes, incorrect efficiency estimates for detecting muon decay, or asyet-unaccounted-for physics. Stronger evidence was presented in 1988 by the Kamiokande experiment, another water Čerenkov experiment. They reported a value of $R^{\prime}$ significantly less than one and also stated that they had rejected all known sources of systematic error related to a detector effect or uncertainty in the atmospheric neutrino fluxes [43] as the cause of the observed deficit. Kamiokande reported that the ratio of ratios, that of $\mu$-like events to $e$-like events between data and MC gave a value of $R^{\prime}=0.59 \pm 0.07$ (stat.).

Following the IMB and Kamiokande results several other experiments studied the flavour ratio of atmospheric neutrinos. The fine-grained iron calorimeters NUSEX [44] and Frejus [45] experiments both reported results that were consistent with MC expectations within their statistics. More recently the Soudan-2 experiment, another fine-grained iron calorimeter experiment, reported a higher statistics result $[46,47]$ that was consistent with the IMB and Kamiokande results. The major breakthrough, which led to an acceptance of neutrino oscillations as the solution to the atmospheric neutrino problem was the 1998 Super-Kamiokande 


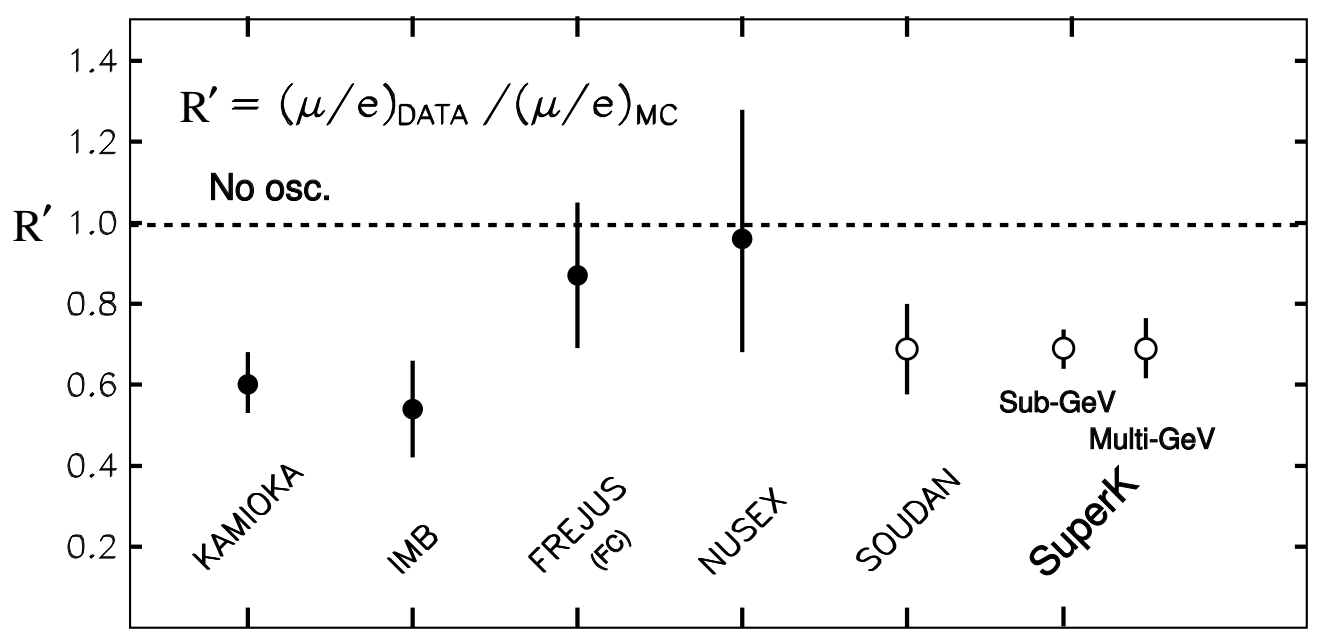

Figure 2.7: Measurements of the atmospheric neutrino flavour ratio of ratios, $R^{\prime}$ (figure from [49]).

result [48]. This result is discussed in detail in the next section (see section 2.3.1 for a description of the Kamiokande and Super-Kamiokande detectors). A summary of the values of $R^{\prime}$ obtained by the atmospheric neutrino experiments is shown in Figure 2.7.

\section{Super-Kamiokande Atmospheric Neutrino Results}

The most persuasive evidence, to date, for atmospheric neutrino oscillations comes from the Super-Kamiokande water Cerenkov experiment. The $22.5 \mathrm{kT}$ fiducial volume of the experiment enabled a large statistical sample of atmospheric neutrinos to be recorded. This enabled the data to be studied as a function of the incoming neutrino energy and direction. Figure 2.8 shows the zenith angle distributions from Super-Kamiokande. The zenith angle distribution of sub-GeV and multi-GeV samples of $\mu$-like and $e$-like events are shown. The black points show the data, the red lines show the MC predictions in the absence of oscillations, and the green lines show the MC predictions of the best fit oscillation scenario. There are a number of important observations to make about these distributions. The $e$-like events agree well with the no-oscillations scenario for both the sub-GeV and multi-GeV samples, whereas, the $\mu$-like events show significant deviations from the no-oscillations scenario. Moreover, the deficit of $\mu$-like events varies with zenith angle and with energy. In the sub$\mathrm{GeV} \mu$-like sample the deficit varies from $\sim 20 \%$ for downward going neutrinos to $\sim 40 \%$ for 

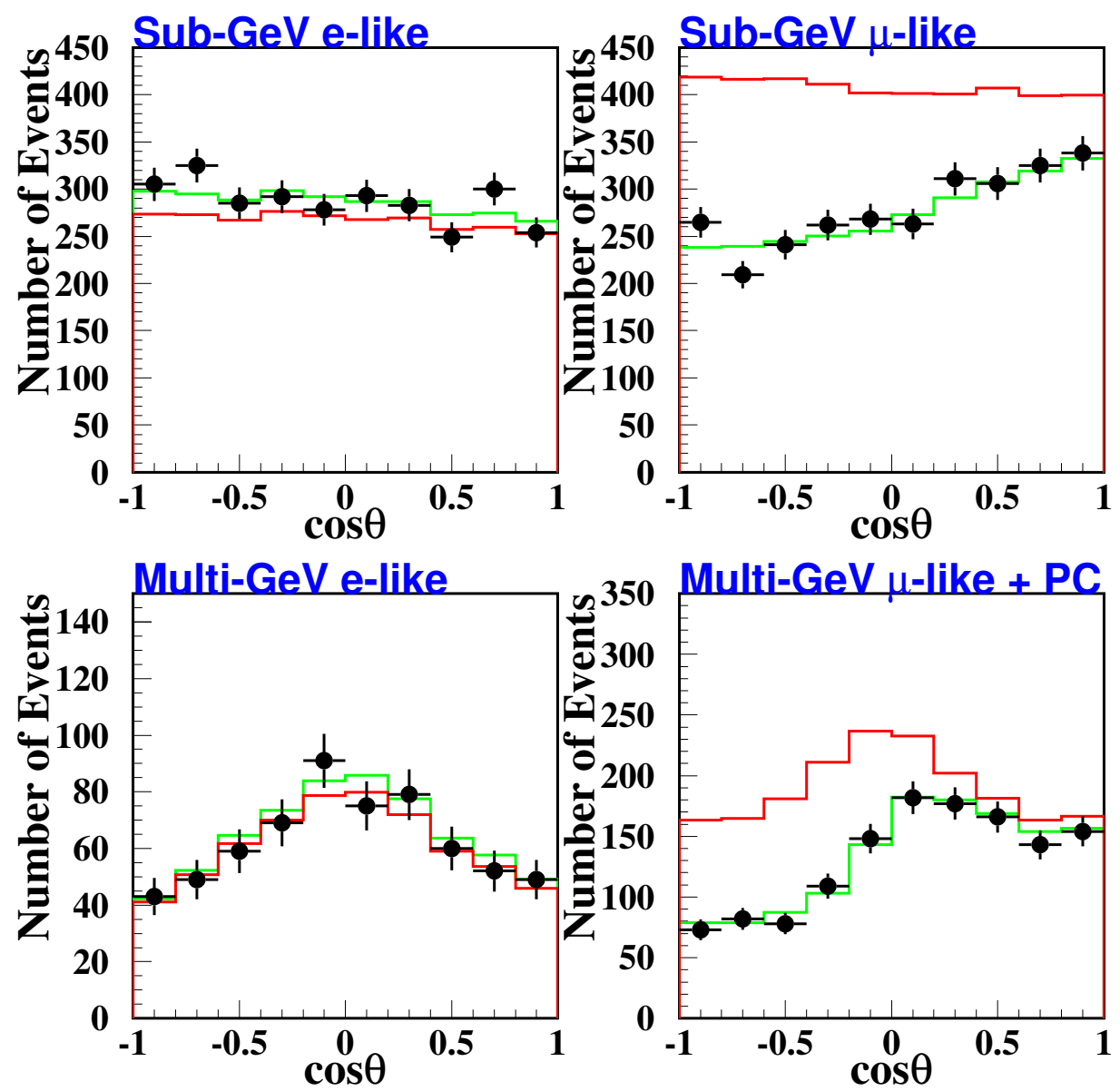

Figure 2.8: Zenith angle distributions from Super-Kamiokande (figure from [50]). The zenith angle distribution of sub-GeV and multi-GeV samples of $\mu$-like and $e$-like events are shown. The black points show the data, the red lines show the MC predictions in the absence of oscillations, and the green lines show the MC predictions of the best fit oscillation scenario. It can be seen that while the $e$-like sample is consistent with no-oscillation, the $\mu$-like sample shows a discrepancy with the no-oscillation scenario that varies with zenith angle. Figure from $[50]$.

upward going neutrinos, while in the multi-GeV sample the deficit varies from around $5 \%$ for downward going neutrinos to $50 \%$ for upward going neutrinos. This variation with energy and distance travelled gives evidence that the muon neutrinos are undergoing oscillations.

In 2004, the Super-Kamiokande collaboration presented a new analysis of their data where they used a selected sample of events with good resolution in $L / E$ [51]. This allowed them to search for the dip in the oscillation probability expected when the argument of the second sine-squared term in equation 2.12 is $\pi / 2$. Figure 2.9 shows the ratio of the observed $\mu$-like events to that expected in the no-oscillation scenario. The black points show 


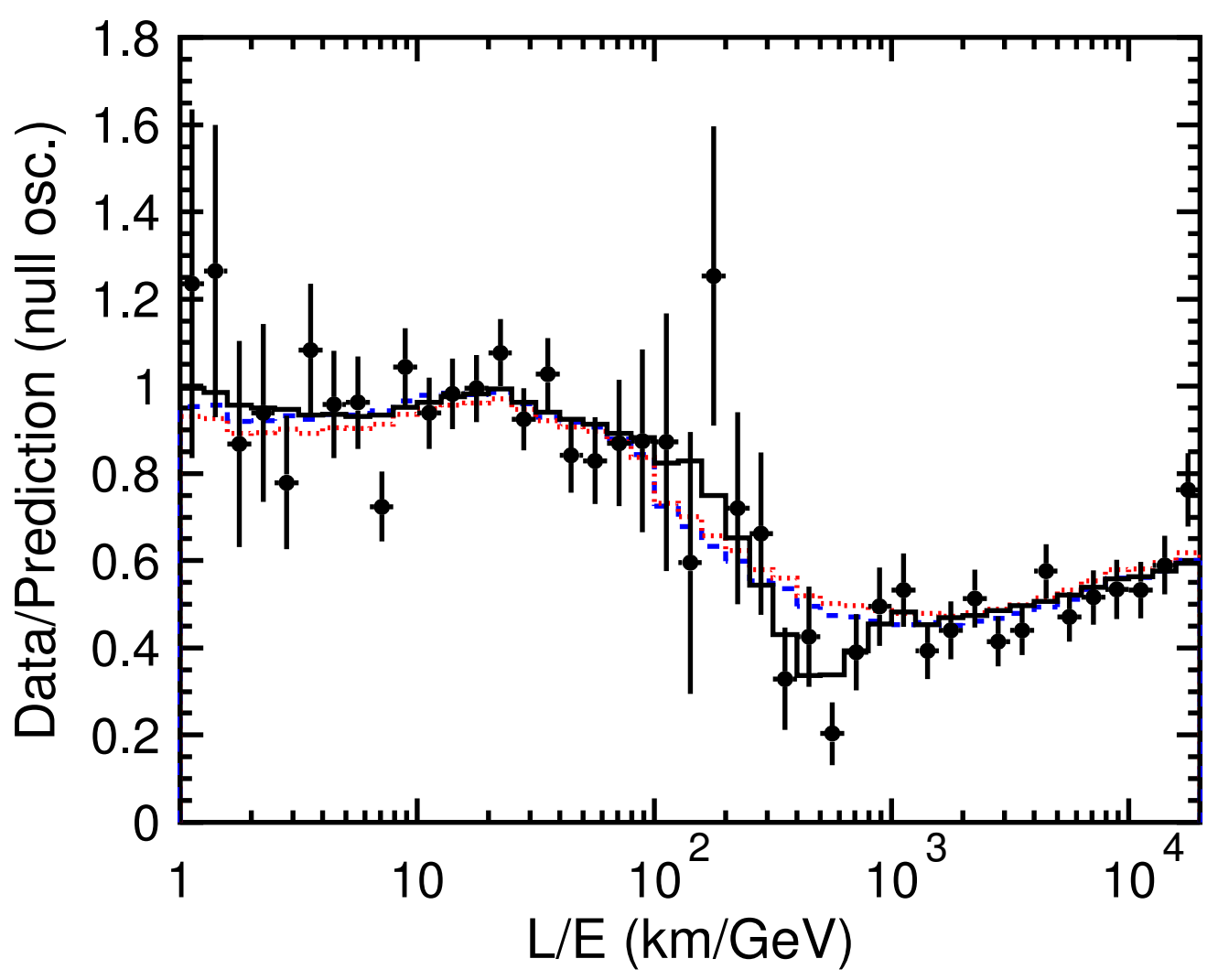

Figure 2.9: Super-Kamiokande observed dependence of neutrino flux with $L / E$ (figure from [51]). The ratio of the observed number of $\mu$-like events to the number expected in the no-oscillation scenario is shown. The black points show the data and the solid black line shows the best fit oscillation scenario. The dotted red line and the dashed blue line show the best fit expectation for neutrino decay and neutrino decoherence respectively.

the data and the solid black line shows the best fit oscillation scenario. The dotted red line and the dashed blue line show the best fit expectation for neutrino decay and neutrino decoherence respectively. For low values of $L / E$ the ratio is one, but for larger values of $L / E$ the ratio drops to $\sim 0.5$. A dip is observed around $L / E=500 \mathrm{~km} / \mathrm{GeV}$, the expected second and higher maximum oscillation points are not expected to be seen because of the $L / E$ resolution of the experiment.

The absence of an excess of $e$-like events leads the Super-Kamiokande collaboration to interpret their result as $\nu_{\mu} \leftrightarrow \nu_{\tau}$ oscillations in a two flavour mixing scheme. Figure 2.10 shows the $68 \%, 90 \%$ and $99 \%$ confidence limit contours for the oscillation parameters obtained from a maximum likelihood fit to their data. The best fit parameters are maximal mixing and $\Delta m^{2}=2.4 \times 10^{-3} \mathrm{eV}^{2}$. 


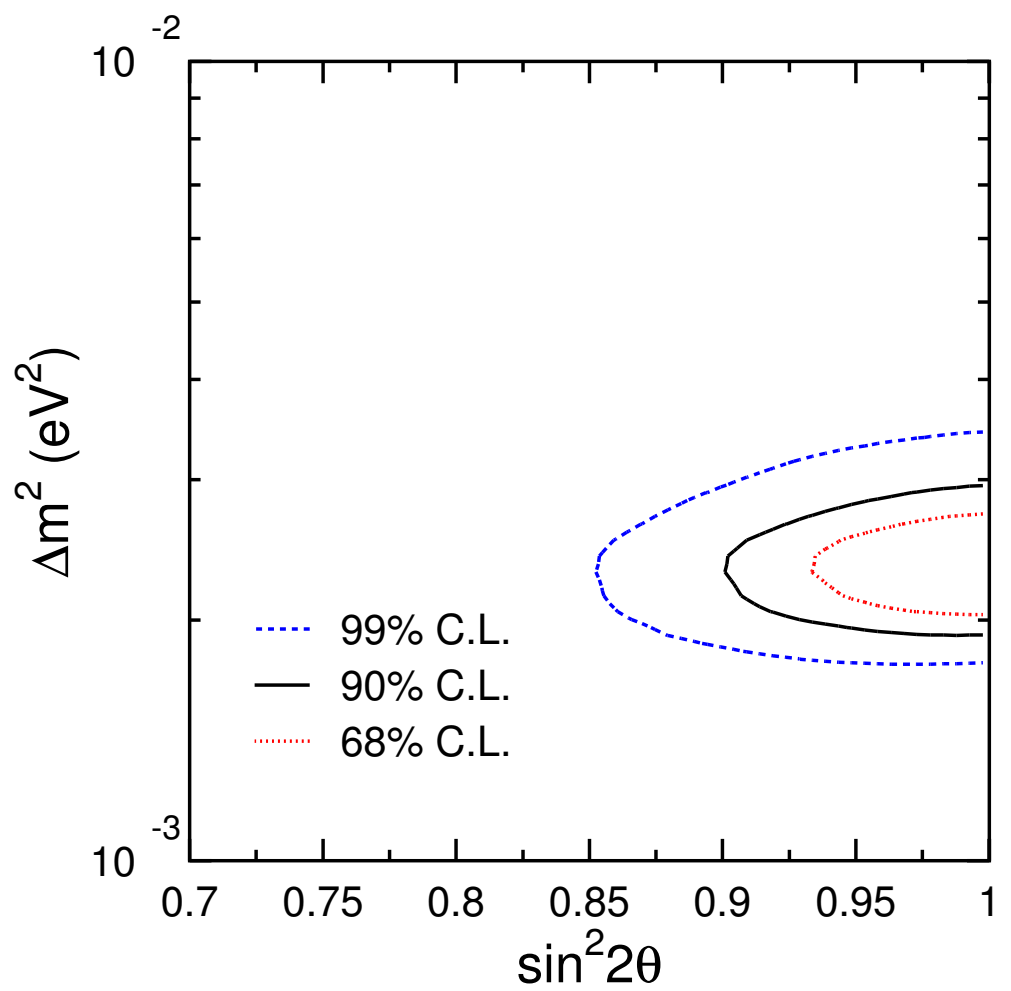

Figure 2.10: Super-Kamiokande allowed regions for atmospheric neutrino oscillation parameters from the $L / E$ analysis (figure from [51]). The contours shown are for $68 \%, 90 \%$ and $99 \%$ confidence limits.

\section{K2K Results}

The K2K (KEK-to-Kamioka) long-baseline accelerator experiment produces a beam of neutrinos with mean energy $\sim 1.3 \mathrm{GeV}$ at the KEK accelerator laboratory. These neutrinos are aimed at the Super-Kamiokande detector in Kamioka, $250 \mathrm{~km}$ away. The K2K experiment report that after achieving $8.9 \times 10^{19}$ protons-on-target the expected number of events occurring in the fiducial volume of Super-Kamiokande is $151_{-10}^{+12}$ (syst.) [52]. However, only 107 events are observed in time with the neutrino beam. 57 of the events are characteristic of CC-QE interactions of $\nu_{\mu}$. The relatively good energy resolution of these 57 events allows an energy spectrum to be created and compared with that measured by the Near detectors close to the neutrino beam source. As expected, an energy dependent disappearance of the $\nu_{\mu}$ events is observed. The results of a fit to both the shape and the number of events yields a result that is consistent with the Super-Kamiokande atmospheric neutrino results. The best fit point for the $\mathrm{K} 2 \mathrm{~K}$ data is maximal mixing with $\Delta m^{2}=2.8 \times 10^{-3} \mathrm{eV}^{2}$. 


\subsubsection{Reactor and Accelerator Neutrinos}

The KamLAND reactor experiment and the K2K accelerator experiment have already been discussed above in the solar and atmospheric sections. However, the picture of neutrino oscillations would not be complete without mention of the CHOOZ and LSND experiments.

\section{Sterile Neutrinos}

The LSND experiment has evidence for $\bar{\nu}_{e}$ appearance in a beam of $\bar{\nu}_{\mu}[9]$. The best fit to the LSND data suggests $\Delta m^{2}=0.2-10 \mathrm{eV}^{2}$, which is significantly higher than the two $\Delta m^{2}$ values associated with the atmospheric and solar neutrino problems. A third $\Delta m^{2}$ requires that there be a 4th species of neutrino. However, the LEP experiments have shown that there are only 3 light, active species of neutrino. Thus, the LSND result implies that there must be a "sterile" neutrino that does not undergo the weak interaction. However, this result is viewed sceptically. Different parties within the LSND collaboration have interpreted the data in conflicting ways. Much of the LSND-allowed region is excluded by the KARMEN [53] and Bugey [54] experiments (see Figure 2.11). A follow-up experiment at Fermilab, called MiniBooNE [10], is expected to confirm or exclude the remaining LSND-allowed regions by late 2005 .

\section{The CHOOZ Experiment and $\theta_{13}$}

Much of the discussion of neutrino oscillations takes place in the simplified 2-flavour framework. However, the sub-dominant oscillation mode involving the $\theta_{13}$ mixing angle is crucial for potentially observing CP violation in the lepton sector, as discussed in section 2.2.1. It is the CHOOZ experiment that has set the most stringent limits on the value of $\theta_{13}$ so far [55]. Located $\sim 1 \mathrm{~km}$ from a nuclear reactor in France, the CHOOZ experiment looks for the disappearance of $\bar{\nu}_{e}$ produced in the reactor. The CHOOZ limit is shown in Figure 2.11. The T2K experiment [56], the proposed Nova experiment [19] and next-generation reactor experiments [57] will have a sensitivity an order of magnitude better than the current limits 
and aim to discover the value of $\theta_{13}$.

\subsubsection{Summary}

There is now strong evidence for neutrino oscillations, both in the atmospheric and solar sectors. The mixing angle and $\Delta m^{2}$ involved in solar neutrino oscillations are known to a $1 \sigma$ accuracy of $\sim 6 \%$ from global fits [58]. The mixing angle and $\Delta m^{2}$ involved in atmospheric oscillations are less well known, they have a $1 \sigma$ accuracy of $\sim 10-15 \%$ from global fits. The third mixing angle $\theta_{13}$ has a lower limit on its value from direct measurements: $\sin ^{2}\left(2 \theta_{13}\right)<$ $0.06-0.2$ at $90 \%$ C.L., depending on the value of $\Delta m^{2}$. Global fits to the oscillation data give a similar value of $\sin ^{2}\left(2 \theta_{13}\right)<0.14$ at $95 \%$ C.L. [58]. A global summary of all the experimental results is shown in Figure 2.11.

\subsection{A Look to the Future}

As we head towards the end of the first decade in the new millennium, experiments such as MINOS will make ever more stringent tests of the oscillation hypothesis and make precision measurements of some of the neutrinos' properties. However, our physical understanding of the neutrino is still far from complete. The ordering of the neutrino masses, the mass hierarchy, is not known fully yet. The last and smallest mixing angle, $\theta_{13}$, is still only known as an upper limit. Hence the potential extent of $\mathrm{CP}$ violation in the neutrino sector and its implications for the evolution of the universe is unknown.

At the same time that precision measurements are being made, the neutrino is further moving from being the scientists' subject of study to being a tool of that study. Experiments are underway to measure geo-neutrinos from within the Earth and hence help determine the evolution and structure of our planet. At the South Pole the IceCube experiment is under construction and when finished it will function as a neutrino telescope, able to see the universe in a new "light". 


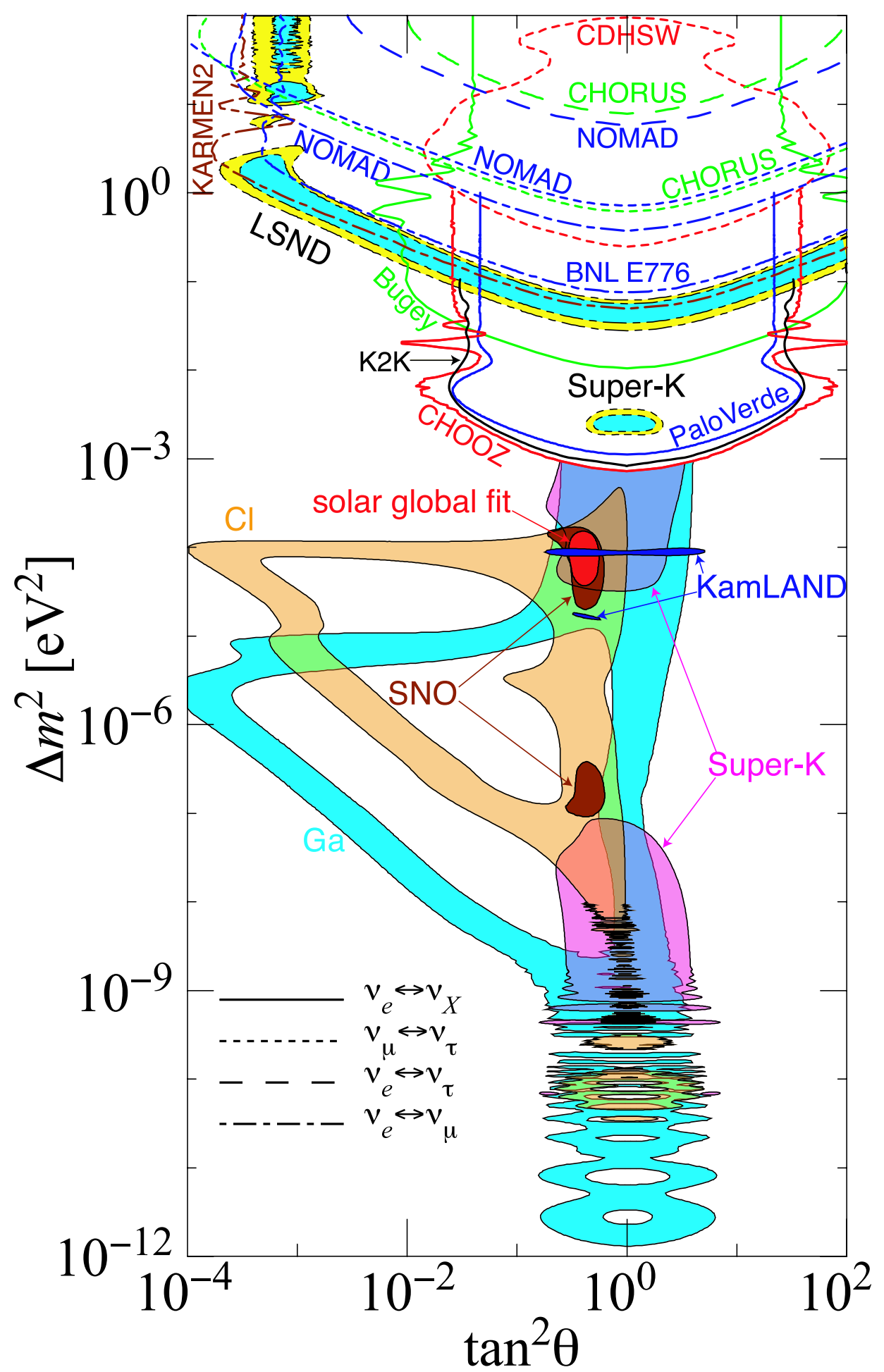

http://hitoshi.berkeley.edu/neutrino

Figure 2.11: Summary of current knowledge of neutrino oscillation parameters (figure from [59]). The coloured regions are allowed regions for the oscillation parameters. The solar results (Cl, Ga, Super-K, SNO, KamLAND) are at 95\% confidence limit; the atmospheric Super-K and LSND results are at 90\% and 99\% confidence limit respectively for the turquoise and yellow areas. White regions demarcated by a line are excluded regions and are at $90 \%$ confidence limit. The line pattern identifies the oscillation channel. 


\section{Chapter 3}

\section{The MINOS Experiment}

MINOS is a long-baseline neutrino oscillation experiment that uses the NuMI neutrino beam created at Fermilab. The neutrino beam is first measured approximately $1 \mathrm{~km}$ downstream of the source using the Near detector. The beam is measured for the second time $735 \mathrm{~km}$ away at the Soudan Underground Laboratory using the Far detector. See Figure 3.1 for the locations of the detectors and the path of the neutrino beam. Comparison of the neutrino energy spectra and flavour composition of the beam at the two detectors will allow measurement of neutrino oscillation parameters. Furthermore, observing the energy dependence of the neutrino disappearance will provide strong evidence for the whole phenomena of neutrino oscillations.

\subsection{The NuMI Neutrino Beam}

The NuMI neutrino beam [60] is created at Fermilab using $120 \mathrm{GeV}$ protons from the Main Injector. Every 1.87 seconds protons are extracted from the Main Injector in a short, approximately $9 \mu$ s spill. The protons are directed at a long, thin, segmented graphite target. In the first year of running $2.0 \times 10^{20}$ protons on target are expected. The secondary particles produced in the target, mainly pions and kaons, are focused (one sign only) by two magnetic horns. The focused beam of secondaries enters a $675 \mathrm{~m}$ long, $2 \mathrm{~m}$ diameter evacuated 


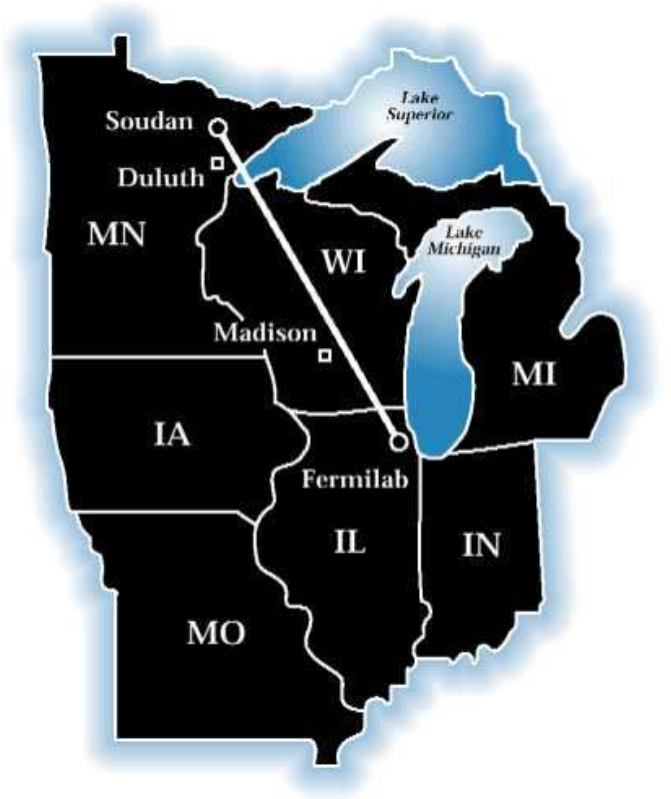

Figure 3.1: Map of neutrino beam used by MINOS. The beam originates at Fermilab in Illinois and travels north-west, through Wisconsin, to the Soudan Underground Laboratory in Minnesota.

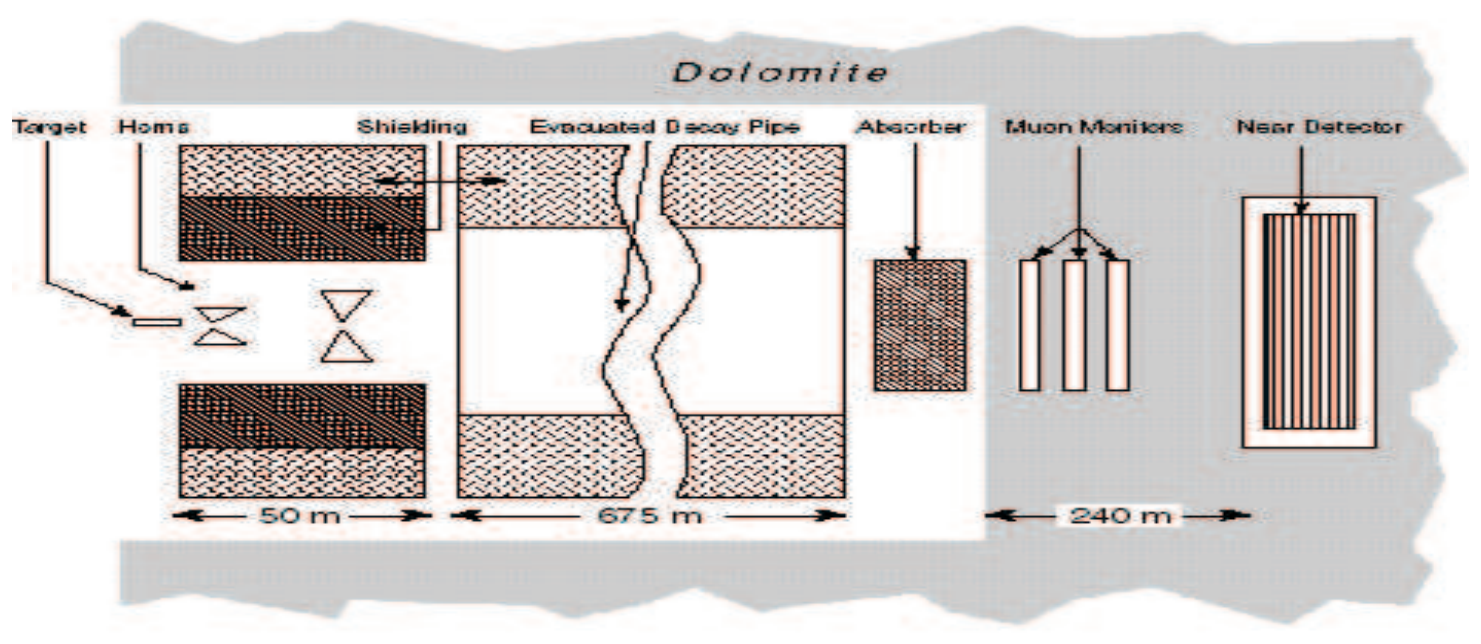

Figure 3.2: Layout of components of neutrino beam (figure from [60]). The beam travels from left to right on the figure. The proton beam strikes the target producing secondary hadrons. The pions and kaons travel down the decay pipe where they decay to produce neutrinos and muons. At the end of the decay pipe a hadron absorber stops any remaining primaries and undecayed secondaries. The muons in the beam are absorbed by the $240 \mathrm{~m}$ of rock between the end of the decay pipe and the Near detector hall. The path of the neutrinos goes through the Near detector and all the way to the Soudan Underground Laboratory in Minnesota. 
beam-pipe. Neutrinos are produced by decays of the secondaries, e.g. $\pi^{+}\left(K^{+}\right) \rightarrow \mu^{+} \nu_{\mu}$. At the end of the beam-pipe there is a water-cooled absorber with an aluminium core encased in steel to stop any remaining primaries and undecayed secondaries. Any muons passing through the absorber are stopped by $240 \mathrm{~m}$ of dense Dolomite rock before they reach the Near detector cavern. The layout of the NuMI beam is shown in Figure 3.2.

The beam has been designed to produce an energy spectrum of neutrinos that is as flexible as possible in order to maximise sensitivity to oscillations. Secondary particles of a given momenta can be selectively focused by varying the magnetic field of the horns as well as by moving the two horns and target relative to each other. Three proposed configurations of the target and horns along with the resulting neutrino energy spectra are shown in Figure 3.3. The beam will initially be run in the so-called low energy configuration since that gives the greatest flux in the oscillation parameter region favoured by Super-Kamiokande. However, this decision will be reconsidered following initial results. In addition, some higher energy running may be necessary to quantify various systematic errors in the measurements.

The flavour composition of the beam will be predominantly $\nu_{\mu}(97.8 \%)$ [61]. Small contributions of $\bar{\nu}_{\mu}(1.8 \%)$ will come from $\mu^{+}$and target-produced $\pi^{-}$decay. The $\nu_{e}$ component of the beam has been minimised as much as possible to allow for appearance searches. However, at the expected maximum in the appearance a $0.4 \% \nu_{e}$ component of the beam remains from $\mu^{+}$and target-produced $K_{e 3}^{+}$decay. The contribution from $\bar{\nu}_{e}$ is small $(0.03 \%)$. In the future, it is conceivable that the direction of the current in the magnetic horns will be reversed to give a beam that is predominantly $\bar{\nu}_{\mu}$.

\subsection{The MINOS Detectors}

The MINOS experiment uses data from three similarly constructed detectors: the Near detector, the Far detector and the Calibration detector (CalDet). The differences between the detectors stem from their different experimental environments. All three detectors are steel-scintillator tracking calorimeters. The Near and Far detectors are also magnetised. In 

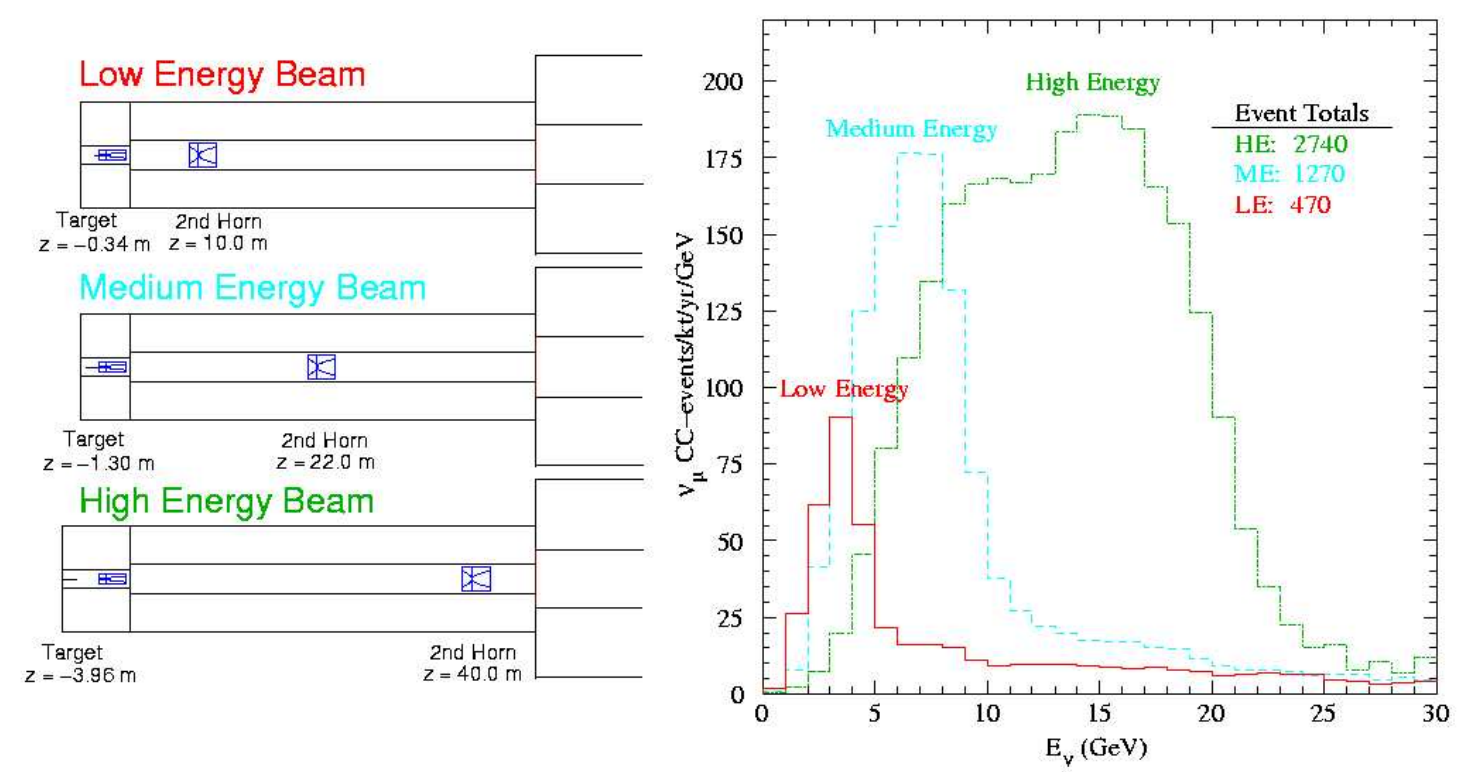

Figure 3.3: Magnetic horn configurations for the three energy configurations and the energy spectra of the beam neutrinos (figure from [62]). The spectra assume $4 \times 10^{20}$ protons on target per year. The first horn is located at $z=0$.

this section the generic features of all three detectors are described before discussing each detector in detail.

The steel and scintillator is arranged into a "sandwich" structure: a layer of scintillator is attached to a layer of steel to form a plane. The detectors are built up from many of these planes, which are hung vertically so as to be perpendicular to the beam neutrinos. The steel in every plane is nominally $2.54 \mathrm{~cm}$ thick $^{1}$ and the scintillator is $1.0 \mathrm{~cm}$ thick. An air gap between each plane of $2.4 \mathrm{~cm}$ means that each steel-air-scintillator unit is $5.94 \mathrm{~cm}$ long.

The detectors use a solid scintillator that is made by extruding polystyrene into long thin strips that are $1.0 \times 4.1 \mathrm{~cm}$ in cross-section (see Figure 3.4) and up to $8 \mathrm{~m}$ long. The orientation of the strips changes by $90^{\circ}$ in each successive plane to give a three-dimensional tracking capability. The polystyrene is doped with the fluors PPO (1\%) and POPOP (0.03\%) that work together to emit blue photons with a mean wavelength of $460 \mathrm{~nm}$. Each scintillator

\footnotetext{
${ }^{1}$ The steel used in the Calibration detector is nominally $2.50 \mathrm{~cm}$ thick, the nearest obtainable standard that could be acquired in Europe. Multiple measurements of the thickness along the edge and centre of a single Calibration detector steel plate gave an average thickness of $2.49 \pm 0.00 \mathrm{~cm}$ with a rms of $0.01 \mathrm{~cm}$ [63]. In addition, repeated measurements of the single steel plate's density gave a result of $7.833 \pm 0.003 \mathrm{~g} / \mathrm{cm}^{3}[64]$. Measurements of Far detector steel from 3 separate heats (batches of steel) have yielded the densities 7.75, 7.82 and $7.70 \mathrm{~g} / \mathrm{cm}^{3}[65]$. Further details of the properties of the MINOS steel are given in [66]. The steel properties at the different detectors are incorporated in to the MINOS MC to ensure a realistic simulation.
} 


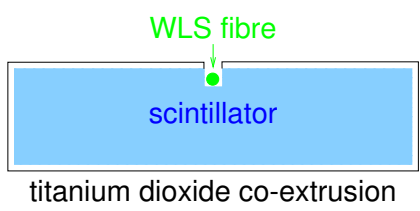

Figure 3.4: Cross-section of scintillator strip. The position of the groove that holds the WLS fibre can be seen in the middle at the top. The strip has a thin layer of co-extruded $\mathrm{TiO}_{2}$ on the outside.

strip has an outer reflective coating $(0.025 \mathrm{~cm}$ thick $)$ containing $\mathrm{TiO}_{2}$ that is mixed with the polystyrene during the extrusion process. The scintillator has an absorption length of $20 \mathrm{~cm}$ hence a technique for extracting the photons from the interaction point is required. A groove runs along the length of every strip into which a $1.2 \mathrm{~mm}$ diameter wavelength shifting (WLS) fibre-optic cable is glued, see Figure 3.5. The blue photons from the scintillator are absorbed in the WLS fibre and re-emitted isotropically. Photons re-emitted along the fibre's central axis are trapped and hence transmitted along the length of the fibre. The WLS fibre shifts the average wavelength of the photons up from blue to green (average $\lambda=530 \mathrm{~nm}$ ). Attenuation along the WLS fibre can be parametrised by a double-exponential form. Typically, the light attenuation has a $1 \mathrm{~m}$ and $7 \mathrm{~m}$ component. Self-absorption of photons in the fluor of the WLS fibre is the cause of the short component.

The scintillator strips are encased in light-tight aluminium $(0.05 \mathrm{~cm}$ thick $)$ modules, which contain between 20 and 28 strips. Figure 3.6 shows the strips inside a scintillator module before the aluminium case was completed. After exiting the strips the WLS fibres run together in a manifold before they terminate in a connector. Clear fibre-optic cables, with a longer attenuation length $(12 \mathrm{~m})$, are attached to each scintillator module and serve to route the photons to multi-anode photomultiplier tubes (MA PMTs).

One of the special features of the MINOS detectors is in the design of their electronics. The Far detector electronics is continuously live. Rather than have the whole detector trigger at once, each PMT can trigger independently. When the dynode signal (sum of the signal from all pixels) from a PMT rises above a threshold of approximately $1 / 3$ of a photoelectron then the electronics digitises that signal. A software trigger running on a small cluster of PCs correlates hits in time from all parts of the detector. Various trigger conditions can 


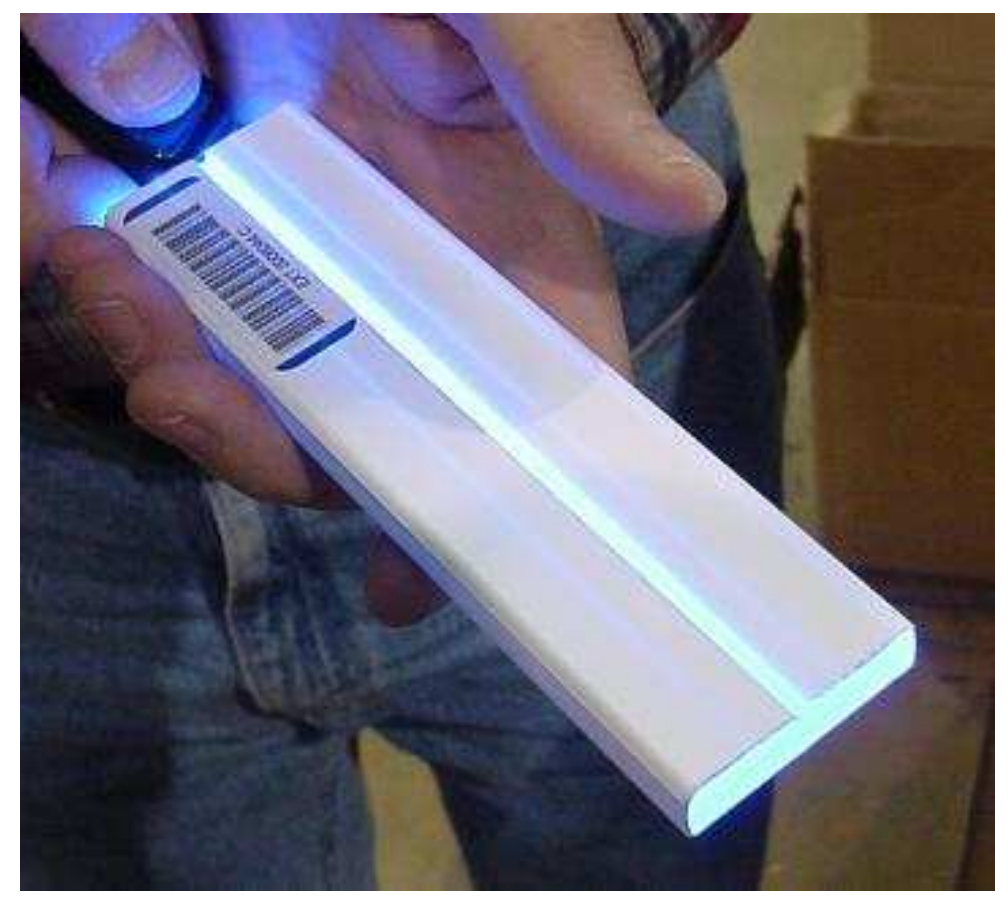

Figure 3.5: A short length of MINOS scintillator illuminated by a blue LED. The groove for the wavelength shifting (WLS) fibre can clearly be seen along the length of the strip. A thin reflective layer of $\mathrm{TiO}_{2}$, on the outside of the strip, is co-extruded with the scintillator to maximise the light collection.

be applied to the time-clustered hits, for example one of the triggers requires 4 out of 5 contiguous planes to be hit. Clusters of hits that fail all the software triggers are discarded, the rest are written to disk. The remarkable feature of the Near detector electronics is that it can digitise an entire $9 \mu s$ beam spill with a time resolution of approximately 19 ns. The Near detector electronics can also be run in a mode similar to the Far detector where it is continuously live. The details of the PMTs and associated electronics used in the Near and Far detectors are described separately below in sections 3.2.1 and 3.2.2.

The magnetic field at the Near and Far detectors is toroidal and has a strength of between 1 to $1.5 \mathrm{~T}$ in the fiducial volume. A coil running down the centre of the detector and back along underneath is used to create the field ${ }^{2}$. The reasons for having a magnetic field are three-fold. Firstly, the magnetic field allows charge separation between the $\mu^{+}$and $\mu^{-}$created in neutrino interactions. Secondly, muon momentum can be measured through curvature in the magnetic field. Thirdly, the field acts to focus the $\mu^{-}$created in the beam

\footnotetext{
${ }^{2}$ At the Near detector the coil is offset from by $50 \mathrm{~cm}$ from the detector centre so as to give a larger fiducial volume around the neutrino beam spot. Also, the coil returns along the side of the detector rather than directly underneath.
} 


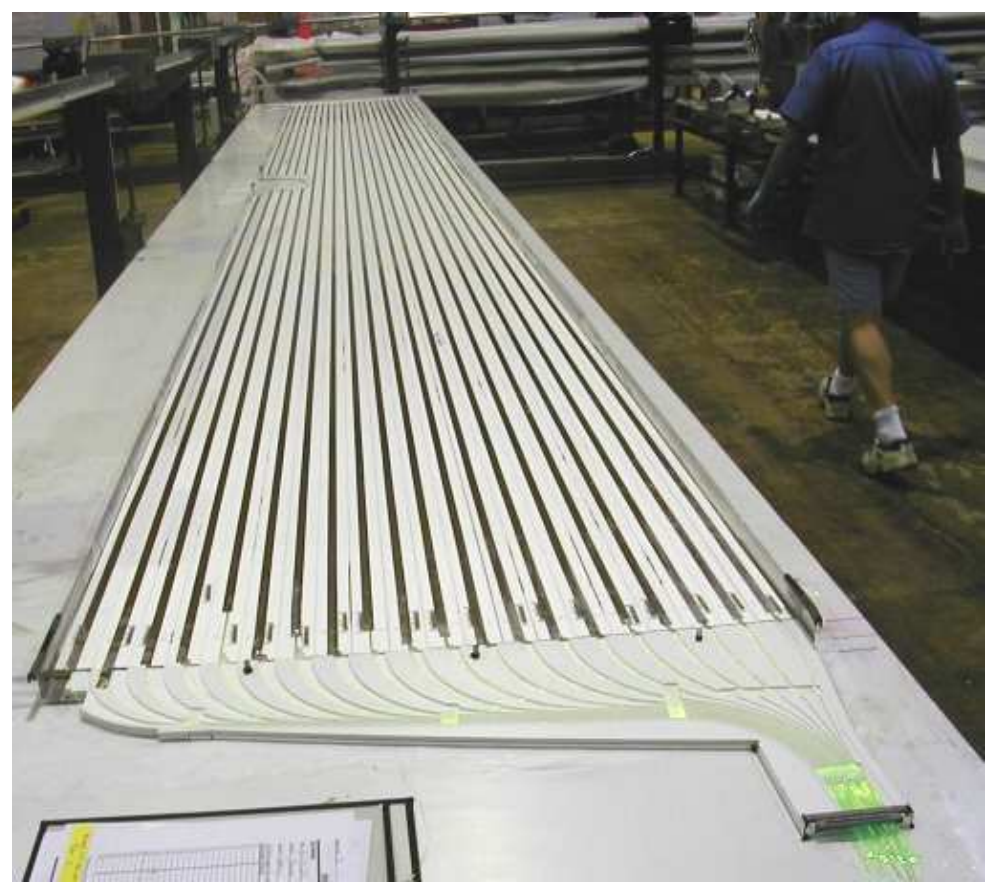

Figure 3.6: Photograph of an open scintillator module. The module is 20 strips wide. In the foreground is the manifold that guides the WLS fibres to the optical connector. The extra lengths of fibre within the manifolds are often referred to as "pigtails".

$\nu_{\mu} \mathrm{CC}$ interactions. The energy resolution on the curvature measurements is between $15 \%$ and $30 \%$ depending on the muon energy and level of containment. Multiple scattering of the muons in the steel is the predominant limitation on the accuracy of these measurements. For muons that stop in the detector a much better measurement of their momenta can be obtained from a range measurement. The energy resolution for stopping muons from a range measurement is approximately $6 \%[67]$.

The calorimetric energy resolution for hadronic and electromagnetic showers has been measured using the Calibration detector. The resolution for hadrons is $\sim 56 \% / \sqrt{E}$ [68][69] and for electrons it is $\sim 21 \% / \sqrt{E}[70]$. Specifically the resolutions have been parameterised $[68,70]$ as:

$$
\begin{array}{ll}
\text { Protons } & (56.6 \pm 0.6) \% / \sqrt{E} \oplus(4.2 \pm 1.4) \% \\
\text { Pions } & (56.1 \pm 0.3) \% / \sqrt{E} \oplus(2.1 \pm 1.5) \% \\
\text { Electrons } & (21.42 \pm 0.06) \% / \sqrt{E} \oplus(4.1 \pm 0.2) \% / E,
\end{array}
$$

where the $\oplus$ symbol indicates that the terms are added in quadrature. The presence of a constant term or $1 / \mathrm{E}$ term in such a parameterisation can occur for a number of 


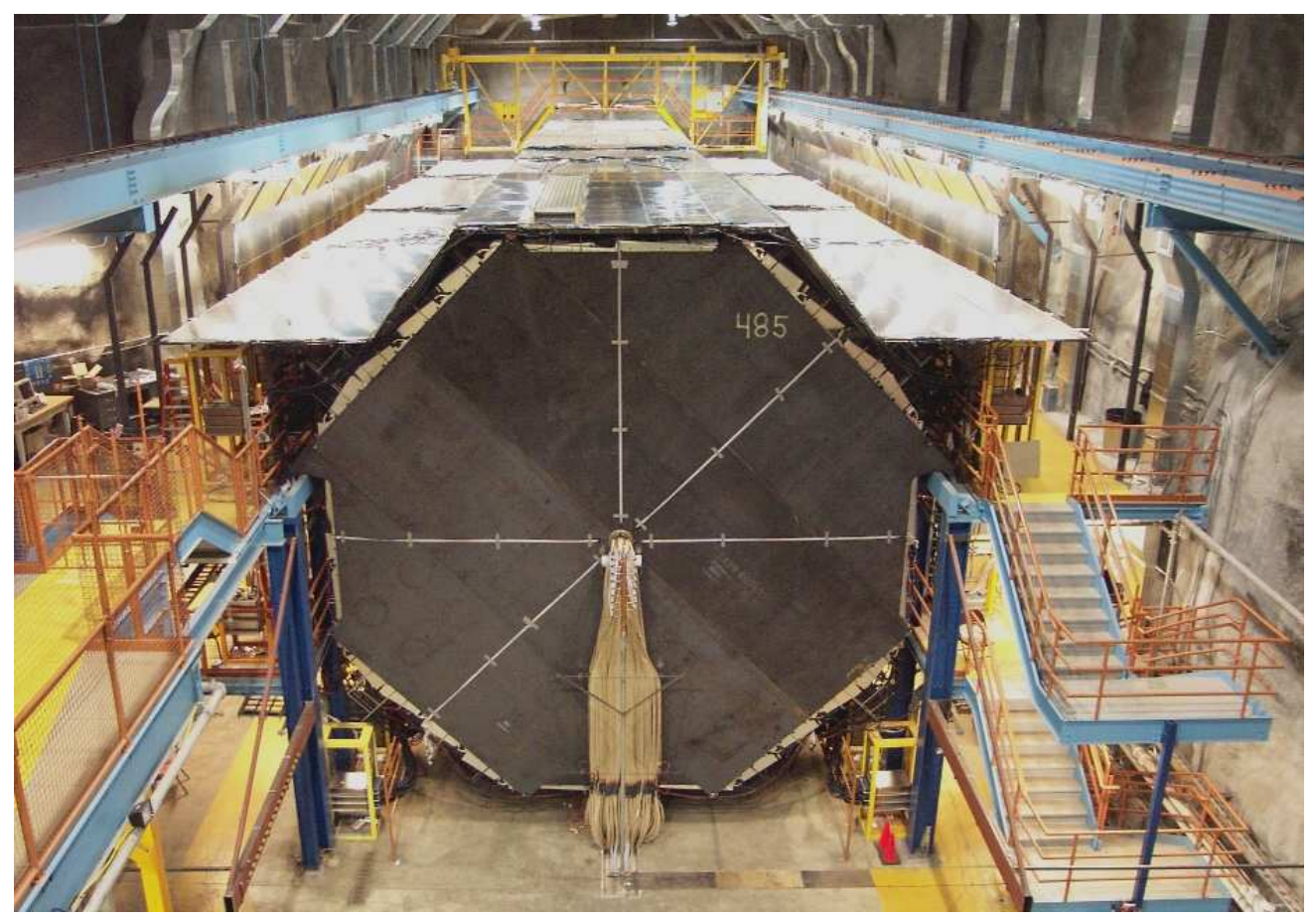

Figure 3.7: Photograph of the Far detector (courtesy of Jerry Meier). The magnetic coil can be seen to exit the centre of the detector and drop down to the floor. The scintillator planes on the top of the detector are a cosmic ray veto shield, vital for the atmospheric neutrino analysis.

reasons $[23,71]$. The dominant contributions to the additional terms are believed to be due to calibration errors, non-uniformities in the detector and noise.

\subsubsection{The Far Detector}

The Far detector with a mass of $5.4 \mathrm{kT}$ is the largest of the three MINOS detectors and is located nearly $1 / 2$ a mile below the Earth's surface at the Soudan Underground Laboratory in Minnesota, USA (see picture in Figure 3.7). The rock above the detector is composed of Greenstone and a high-purity iron ore which gives an overburden of $2070 \mathrm{~m}$ water equivalent. The detector is constructed from 486 octagonal planes that measure $8 \mathrm{~m}$ across. The first 249 planes make up a so-called super-module then after a gap of approximately $1.2 \mathrm{~m}$ a second super-module of 237 planes starts. The division of the detector in to two super-modules is made due to restrictions on the length of the magnetic coil, thus each super-module is independently magnetised. 
Every plane in the Far detector has 192 scintillator strips that are oriented at $45^{\circ}$ to the horizontal. The strips are read out at both ends by multi-anode, 16 pixel Hamamatsu M16 PMTs. Given the large number of channels (185856 strip-ends) in the Far detector, a multiplexing ${ }^{3}$ technique is employed to reduce the amount of instrumentation required. Multiplexing the signals from many strips in a plane is possible because the transverse spread of hadronic/electromagnetic showers from beam neutrinos is limited to a $\sim 1 \mathrm{~m}$ region. Therefore, 8 strips in a plane can be read out by the same PMT pixel with a minimal impact on event reconstruction ability. To enable determination of which strip was actually hit, the 8 strips read out by a single pixel on one side of the detector are read out by 8 different pixels on the other side. The complex optical-wiring needed to perform the multiplexing is done within a so-called MUX box. Each MUX box contains three PMTs and reads out one side of two planes.

The front-end electronics at the Far detector is based around a VA chip, which is a version of the Viking chip made by IDE AS of Norway. Each VA chip samples and holds the signals from a single PMT. The digitisation, pedestal subtraction and sparsification takes place at the VARC (VA Readout Controller) [72]. Data from the VARC are read out over VME by a ROP (Readout Processor) where it is then transmitted to the DAQ PCs via the PVIC interface [73].

A cosmic ray veto shield has been constructed around the top and sides of the Far detector, see Figure 3.7. This veto shield is vital for the rejection of backgrounds in the analysis of atmospheric neutrino data. Design of the MINOS detectors was optimised for beam neutrinos hence the planes were positioned vertically. A large source of background for the atmospheric neutrino analysis is caused by cosmic muons coming down between the active scintillator planes and only interacting once inside the fiducial volume. The shield allows such cosmic muons to be tagged with a high efficiency and hence removed from the analysis.

\footnotetext{
${ }^{3}$ Strictly speaking, the multiplexing is actually optical summing.
} 


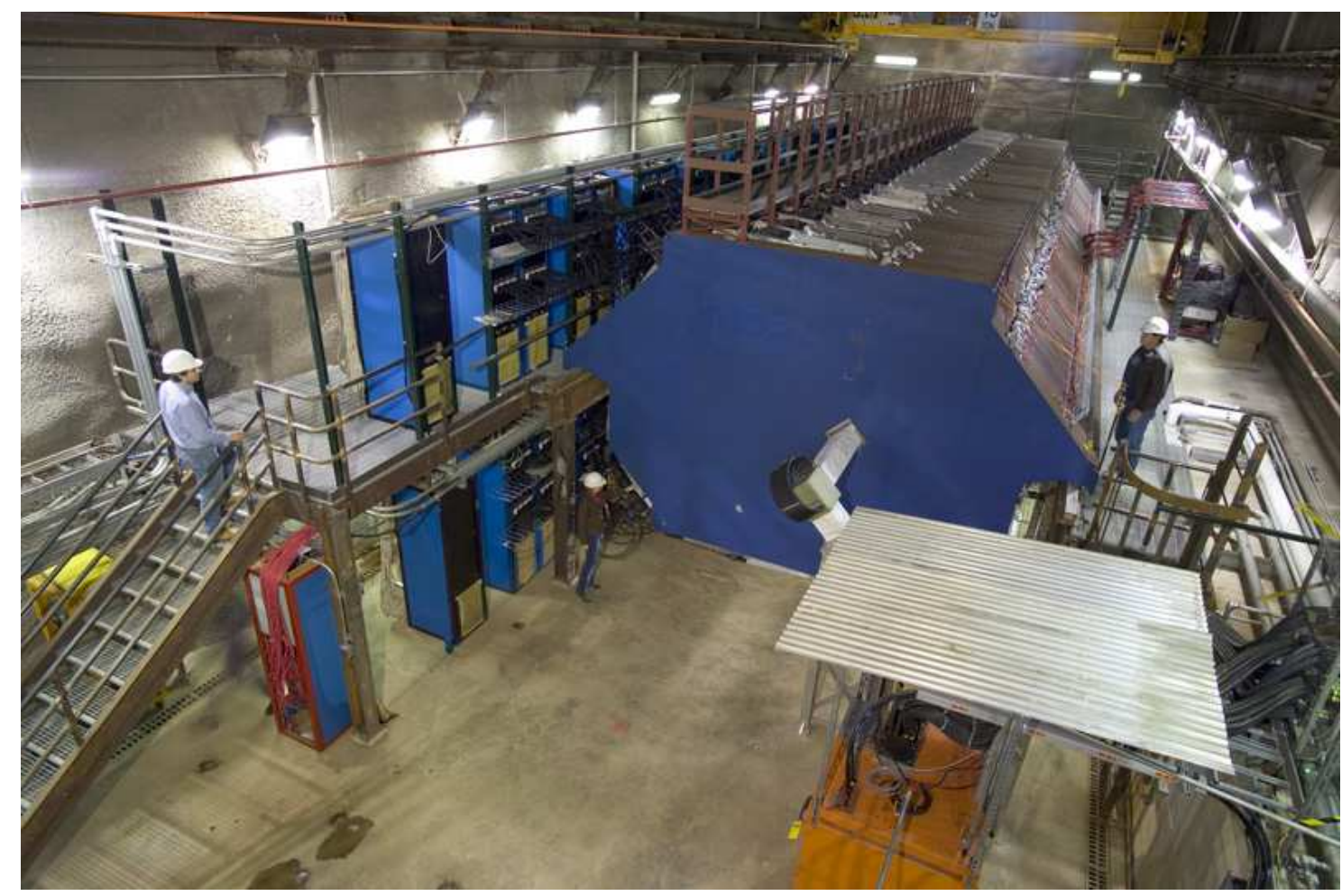

Figure 3.8: Photograph of the Near Detector (courtesy of Fermilab Visual Media Services). The racks along the left-hand side hold the electronics and the MA PMTs. The magnetic coil can be seen to extend from the front of the detector and drop diagonally down to the right side of the detector.

\subsubsection{The Near Detector}

The Near detector is approximately $1 / 5$ th the size of the Far detector with a mass of $0.98 \mathrm{kT}$ and is located on-site at Fermilab, $98 \mathrm{~m}$ below the surface (see picture in Figure 3.8). Design of the Near detector was strongly influenced by the small neutrino beam spot and the high event rate. When the neutrino beam hits the Near detector it has a diameter of only $50 \mathrm{~cm}$ compared to $1 \mathrm{~km}$ at the Far detector. This means means that the Near detector's fiducial volume can be centred around the beam spot.

In order to contain high energy muons the Near detector is 282 planes long. The height and width of the detector are driven by two physical needs: firstly, a fiducial volume around the beam spot big enough to contain the neutrino interactions of interest; and secondly, that enough steel is present to create a magnetic field as similar as possible to the Far detector. Combining these needs has led to the design of an elongated octagon shape, measuring $3.8 \mathrm{~m}$ tall and $4.8 \mathrm{~m}$ wide. Furthermore, cost considerations meant the regions away from the beam 


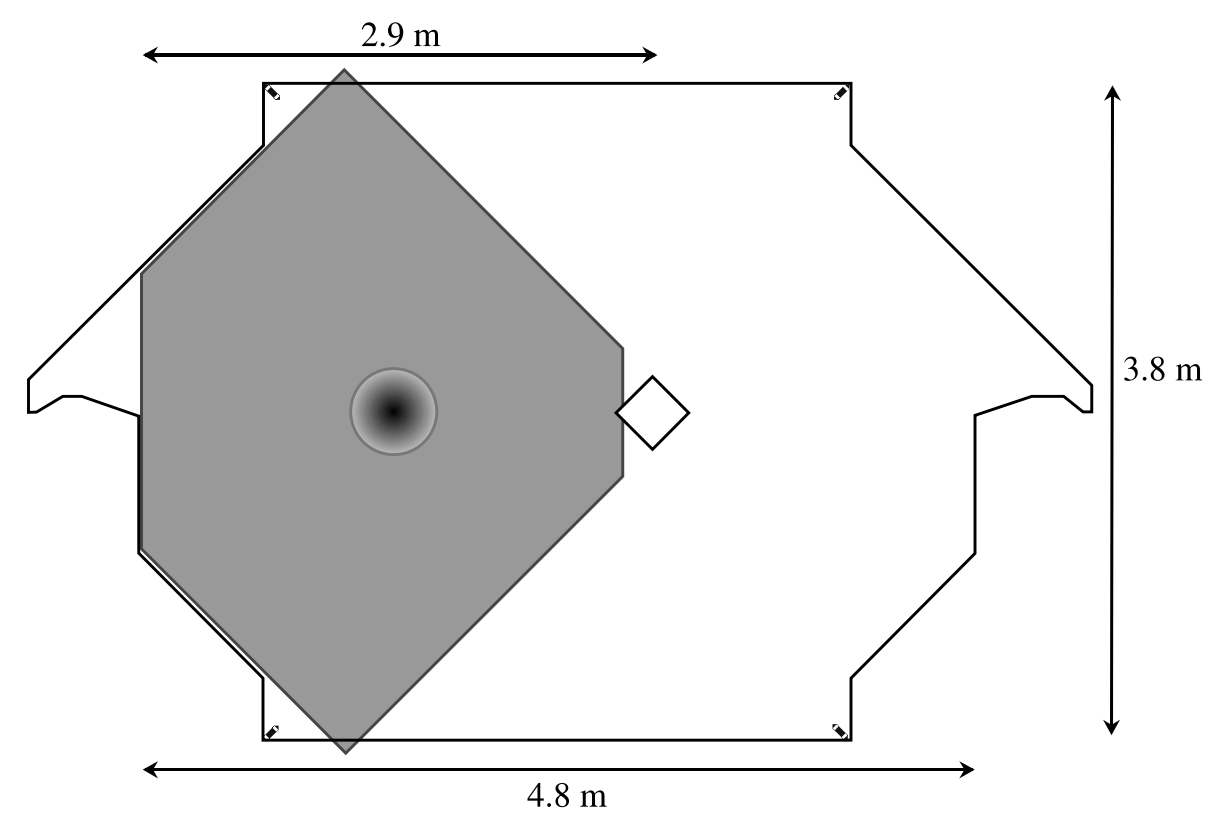

Figure 3.9: Sketch of a partially-instrumented Near Detector plane (figure from [74]). The light grey, shaded area shows the location of the scintillator. The dark circle indicates the $50 \mathrm{~cm}$ diameter beam spot and the square represents the magnet coil hole.

spot were not instrumented in every plane. In fact, only every 5 th plane is fully-instrumented as this is enough to indicate the presence of tracks and interactions outside the central beam area. The design of a partially-instrumented plane is shown in Figure 3.9. It can be seen that the coil hole is offset from the detector centre by $50 \mathrm{~cm}$ and that the beam spot is also offset but by $50 \mathrm{~cm}$ in the opposite direction.

The Near detector is divided into four logical sections [74]: veto, target, calorimeter and muon-spectrometer as shown in Figure 3.10. Planes 0-20 make up the veto section, which is there to prevent biases from neutrons produced by the neutrino-beam in the rock upstream and any possible end effects. The target section consists of 40 planes (21-60) and only neutrinos interacting in this region will be used in the near/far comparison. To fully contain the hadronic showers from the neutrino interactions 60 planes (61-120) make up the calorimeter section. Lastly, to measure the momenta of high energy muons there is a muonspectrometer section consisting of 161 planes (121-281). Every plane in the forward section (veto, target and calorimeter) is instrumented to enable accurate tracking and calorimetry. However, in the muon-spectrometer only one in every five planes is instrumented since it is not necessary to know the exact range of long muons. 


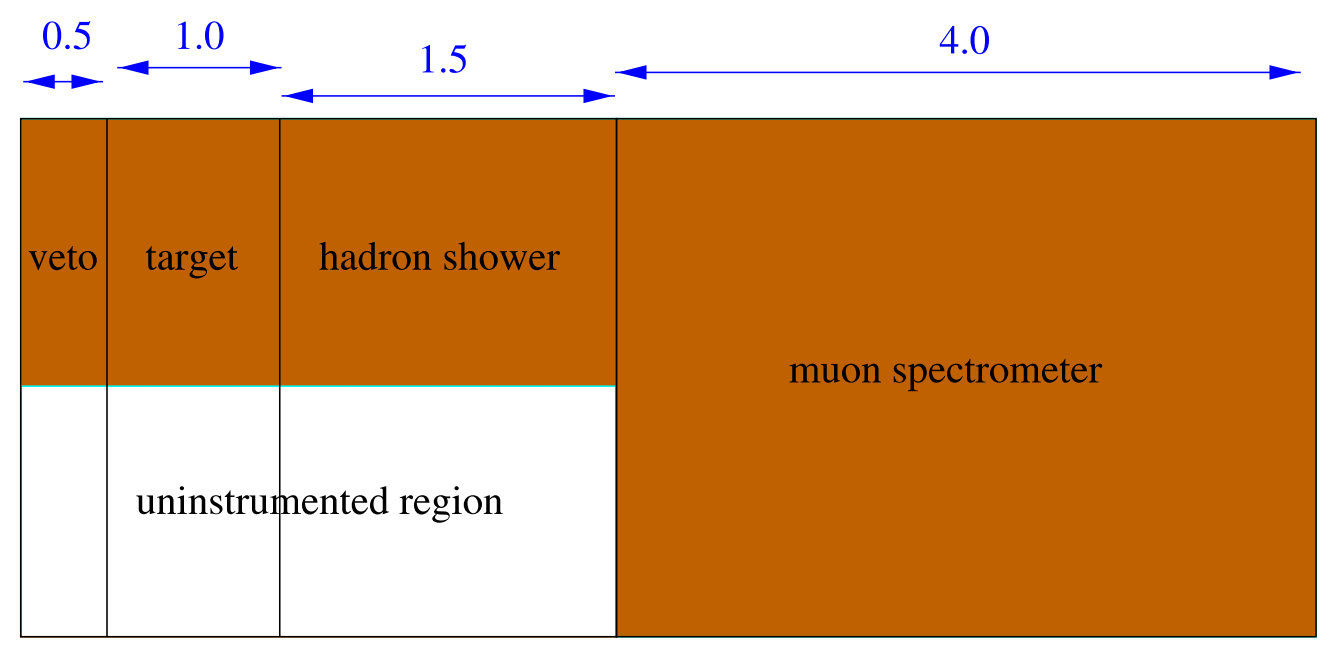

Figure 3.10: Four logical sections of the Near Detector (figure from [74]). The location of the veto, target, hadron calorimeter, and muon spectrometer detector sections are shown. The length of each section is shown and correspond to the thickness of steel in metres. The beam impinges from the left and is centred on the shaded region. The "uninstrumented" region only has scintillator in every 5 th plane.

In contrast to the Far detector, scintillator strips in the Near detector need only be read out on one end; their relatively short lengths (typically $2.8 \mathrm{~m}$ compared to $8 \mathrm{~m}$ at the Far detector) ensure that enough photons reach the PMTs to detect the passage of a minimum ionising particle efficiently. To boost the light levels a reflective mylar coating is applied to one end of each strip and WLS fibre.

Multi-anode Hamamatsu M64 PMTs with 64 pixels are used to read out the scintillator strips in the Near detector. A partially-instrumented plane has 64 strips so is read out by a single PMT. The fully-instrumented planes have 96 strips so $1 \frac{1}{2}$ PMTs are required to read them out. Furthermore, to reduce the instrumentation required, a 4-fold electrical summing technique is employed in the muon-spectrometer region. Each PMT is split into groups of 4 pixels and the signals from the 4 anodes are fed into a single electronics channel. The consequence of the electrical summing is that a "seed" track in the forward region is needed to project into the muon-spectrometer for unambiguous reconstruction.

Due to the multiple events occurring in each beam spill, deadtime-less, high-speed frontend electronics is required for the Near detector. A system based on the multi-ranging QIE chip used in the CDF and KTEV experiments has been developed [75]. Charge from the PMTs is divided into 19 ns time windows, called buckets, and stored in a FIFO. After each 
$9 \mu s$ beam spill the buckets are read out and sparsified individually before being passed to the DAQ. The electronics can also operate in a dynode triggered mode where only 8 buckets of data are stored before being read out. The dynode trigger mode is used to take cosmic ray data. A detailed description of the Near detector electronics is given in $[76,75]$.

\subsubsection{The Calibration Detector}

The Calibration detector (CalDet) is the smallest of the three detectors with a mass of only 12 tonnes, see Figure 3.11. The essential role of CalDet in the MINOS experiment was to measure the response and topology of hadronic and electromagnetic interactions in the few-GeV regime. During the years 2001-2003 CalDet was exposed to beams of $p^{+}, \pi^{ \pm}, e^{ \pm}, \mu^{ \pm}$ between $0.4-10 \mathrm{GeV}$ at the CERN PS accelerator East Area test-beam hall. Specifically, data were taken in the $\mathrm{T} 7$ and $\mathrm{T} 11$ test-beams [68]; the $\mathrm{T} 7$ test-beam provides beams of particles in the range of momenta $0.5-10 \mathrm{GeV} / \mathrm{c}$, whereas the T11 test-beam operates at lower momenta in the range $0.4-3.6 \mathrm{GeV} / \mathrm{c}$.

CalDet can be thought of as a miniature cut-out of the Near or Far detector as it has only 60 planes that measure $1 \mathrm{~m}$ square with 24 strips per plane. However, the strips run horizontally and vertically rather than being canted at $45^{\circ}$. One other significant difference is that there is no magnetic field in CalDet so measurements of muon momentum by curvature are not possible.

Instrumentation and read out of CalDet was designed to simulate the Near and Far detectors as much as possible. Long fibre-optic cables, both clear and WLS, were attached to the scintillator modules to more closely match the lengths used in the Near and Far detectors. The first CalDet data was taken using only Far detector instrumentation (frontend electronics and PMTs) but later data taking used only Near detector instrumentation. When running with just Near detector instrumentation, one end of each strip was fitted with a mylar reflector-connector to simulate the one-ended read out used in the Near detector. In addition, there was substantial data taken in a mixed, hybrid mode where one end of each strip was read out using Far detector instrumentation and the opposite end by Near detector 


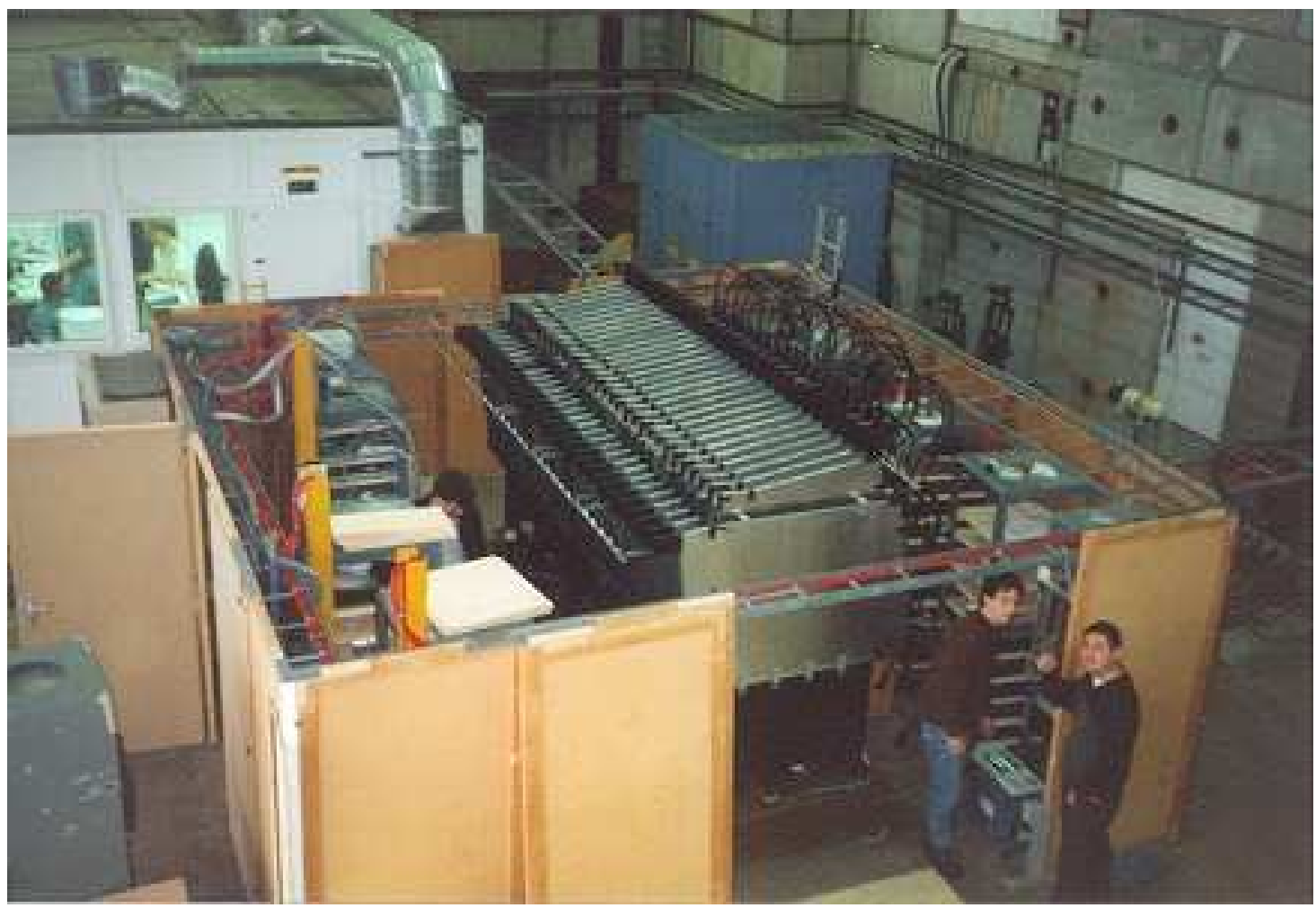

Figure 3.11: Photograph of the Calibration detector. The detector is shown during assembly in its enclosure outside of the test-beam. The alternating orientation of the planes can be seen.

instrumentation. By exposing the two sets of instrumentation to identical interactions, on an event-by-event basis, the level of various potential systematic differences between the two sets of instrumentation could be determined [76].

During test-beam running CalDet was externally triggered so as to efficiently record the events of interest. Although, when recording cosmic events the usual dynode trigger system of the PMTs was used. A time-of-flight (TOF) system with two widely separated scintillator paddles was used, both to measure the speed of the particles and as the external trigger. Čerenkov counters were used to tag the electrons.

A number of MINOS theses have described the Calibration detector, the test-beams as well as much other detail on the whole experimental setup [68, 70, 76, 38, 77, 18, 69]. 


\subsubsection{Detector Co-ordinate systems}

The rotation of the scintillator strips by $45^{\circ}$ from the horizontal and vertical in the Near and Far detectors suggests the use of a more natural co-ordinate system than the standard definitions of XYZ [78]. A U-axis and a V-axis are defined such that each scintillator plane directly measures positions along one of the axes, as shown in Figure 3.12. The origin of the U-axis and the $\mathrm{V}$-axis is located at the centre of the coil hole in the Near and the Far detectors, and simply at the detector centre in the Calibration detector.

The coordinate systems used by MINOS are right-handed. The positive $\mathrm{z}$ direction is the horizontal component of the beam direction and thus points approximately north-west. In addition to the UVZ co-ordinate system a XYZ system is also defined for display purposes and for relation to the outside world. The y-axis is vertical and points straight up. The $\mathrm{x}$-axis is horizontal and points approximately south-west, or left relative to the neutrino beam when looking downstream. The relation between the different co-ordinates is shown graphically in Figure 3.12 .

The axis of symmetry in the MINOS detectors is the z-axis. All three detectors have a rotational symmetry about the z-axis in the fiducial volume. As such, a useful parameter when evaluating the detector characteristics is $\operatorname{Cos}\left(\theta_{z}\right)$, the angle subtended from the z-axis. A particle with a fixed $\operatorname{Cos}\left(\theta_{z}\right)$ travels the same distance through a steel plane independent of it's coordinates in $\mathrm{X}$ and $\mathrm{Y}$. In the $\mathrm{XYZ}$ system $\operatorname{Cos}\left(\theta_{z}\right)$ is defined as:

$$
\operatorname{Cos}\left(\theta_{z}\right)=\frac{z_{2}-z_{1}}{\sqrt{\left(x_{2}-x_{1}\right)^{2}+\left(y_{2}-y_{1}\right)^{2}+\left(z_{2}-z_{1}\right)^{2}}}
$$

where $\left(x_{1}, y_{1}, z_{1}\right)$ and $\left(x_{2}, y_{2}, z_{2}\right)$ are the coordinates of points on a track. Similarly, the gradient in the UZ and VZ planes can be combined:

$$
\operatorname{Cos}\left(\theta_{z}\right)=\frac{1}{\sqrt{m_{u z}^{2}+m_{v z}^{2}+1}}
$$

where, assuming a straight line, $m_{u z}=\frac{u_{2}-u_{1}}{z_{2}-z_{1}}, m_{v z}=\frac{v_{2}-v_{1}}{z_{2}-z_{1}}$ for the points on a track $\left(u_{1}, v_{1}, z_{1}\right)$ 


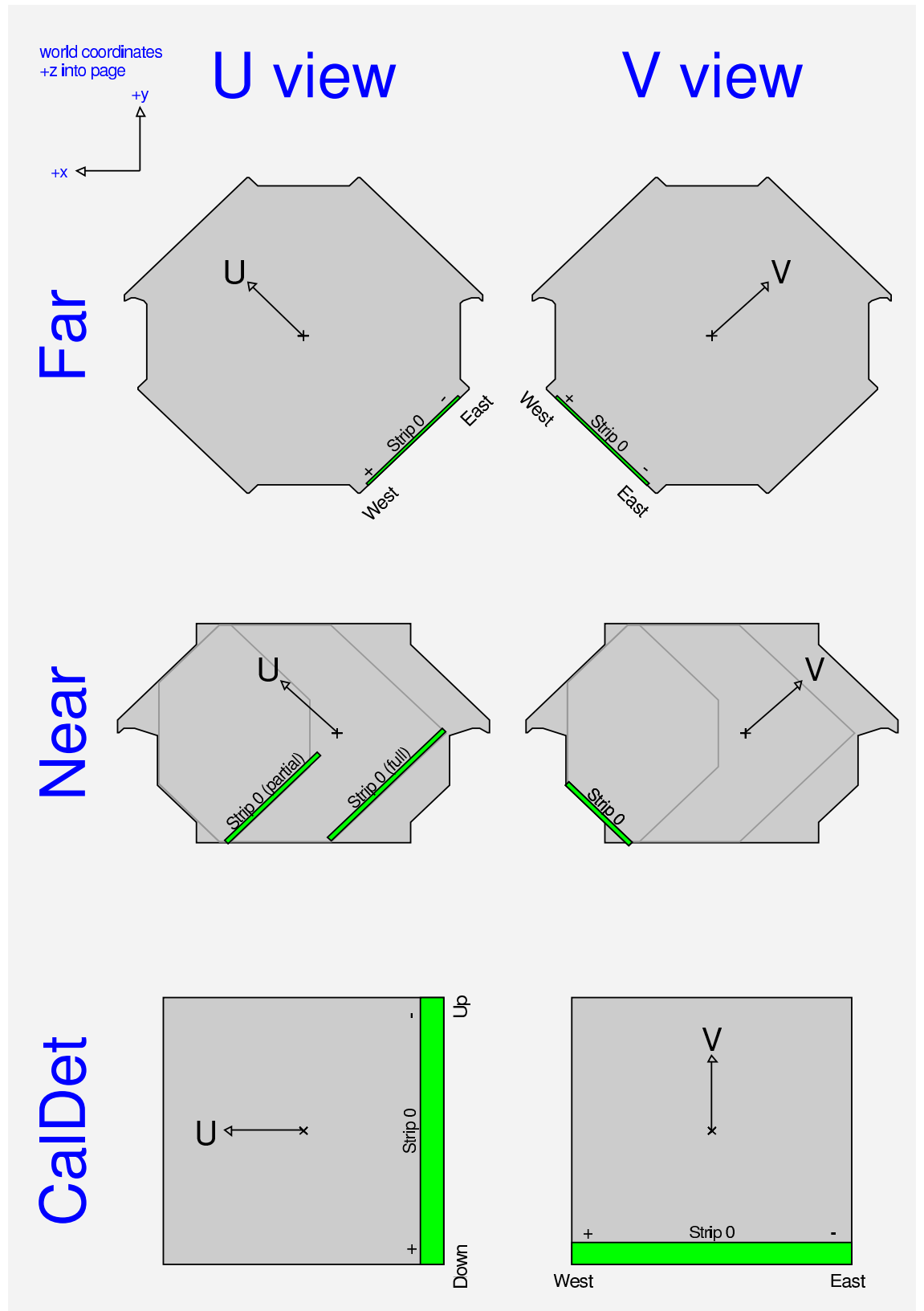

Figure 3.12: MINOS coordinate systems (figure from [78]). The definition of the U-axis and $\mathrm{V}$-axis for the different detectors and their relation to the XY system are shown. 
and $\left(u_{2}, v_{2}, z_{2}\right)$.

\subsection{Physics Capabilities}

The goals of the MINOS experiment are to provide strong evidence that neutrino oscillations exist in the region of parameter space suggested by the atmospheric neutrino experiments and to make a precision measurement of the parameters. MINOS was designed to provide several independent and parallel measurements, each of which is capable by itself of addressing neutrino oscillations [74]. These measurements fall into three groups: statistical tests of the oscillation hypothesis, spectral dependence measurements and appearance searches.

The basic approach of the MINOS oscillation analyses is to separate charged current (CC) events from neutral current (NC) events. Broadly speaking, for $\nu_{\mu}$ interactions this can be achieved by separating the events into "short" and "long" categories. The long events being caused by the high penetrating power of the muon and the short events by the showering of hadrons and electrons.

The measurements MINOS aims to make are based around looking for changes in the neutrino beam, thus it is necessary to understand the neutrino beam at the source. The production of the neutrino beam in the NuMI beam-line is only predicted a priori to between $10-20 \%$ [60]. One of the ways in which systematic errors on the measurements are controlled and estimated is by using the Near detector. For example, by measurement of the neutrino spectrum; and by estimating the contamination of $\nu_{e}$ events. In fact, to obtain the expected spectrum of neutrinos at the Far detector (without oscillation effects) it is necessary to extrapolate the measurements made at the Near detector (since the Near detector "sees" a larger solid angle of the neutrino beam). It is estimated that by a process of feeding back the Near detector measurements to the NuMI beam-line simulation this can be achieved to a precision of $2 \%[60]$. 


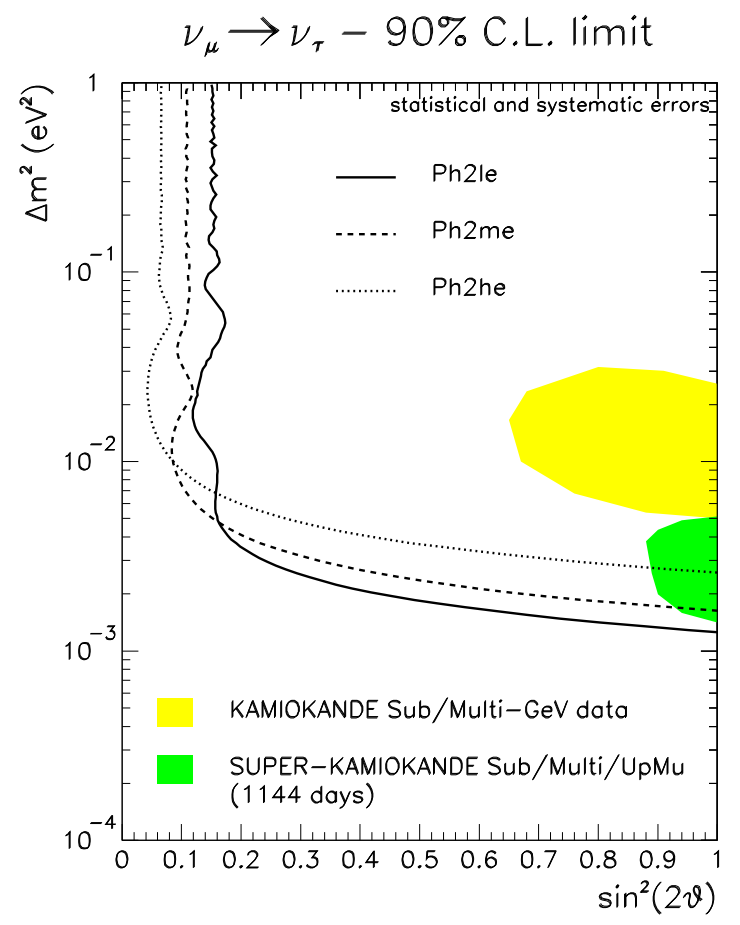

Figure 3.13: Limits on oscillation parameters using the T-test (figure from [79]). 90\% confidence limits on $\nu_{\mu} \rightarrow \nu_{x}$ oscillation parameters using the T-test are shown for low, medium and high energy beam configurations (Ph2le, $\mathrm{Ph} 2 \mathrm{me}$ and $\mathrm{Ph} 2$ he respectively). The limits shown are those that will be obtained after running for 4 years with $2 \times 10^{20}$ protons on target per year. The allowed regions for Kamiokande and Super-Kamiokande are shown for comparison.

\subsubsection{The T-Test}

The T-Test is a simple hypothesis test: that of oscillations versus no oscillations. A measurement of the ratio of $\nu_{\mu} \mathrm{CC}$ (or "long") events to all events is made in both the Near and Far detectors. A significant difference between the two ratios is evidence of neutrino oscillations. The region of parameter space for $\nu_{\mu} \rightarrow \nu_{x}$ oscillations that MINOS should be able to exclude using this technique is shown in Figure 3.13. There are several advantages to this sort of statistical test: it does not depend on any normalisation of the neutrino flux, nor does it depend on a precise energy measurement of each event. However, this method can only determine the presence of oscillations, it cannot make a measurement of the oscillation parameters. 
CC energy distributions - Ph2le, $10 \mathrm{kt} . y \mathrm{r}, \sin ^{2}(2 v)=0.9$
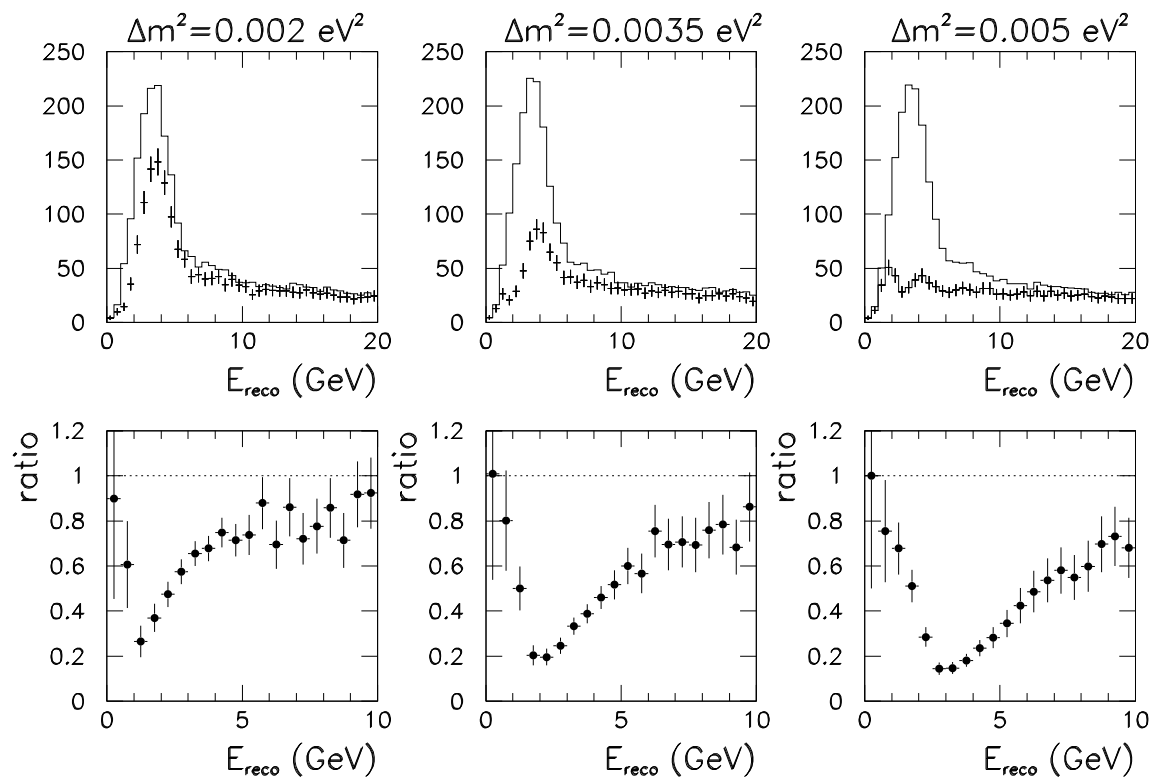

Figure 3.14: Parameter determination using $\nu_{\mu}$ CC energy spectra (figure from [80]). Three different values of $\Delta m^{2}$ are used along with $\sin ^{2} 2 \theta=0.9$. In the top row of plots, the solid line shows the CC energy spectrum expected in the Far Detector for no oscillations, and the crosses show the spectrum that would be measured for the given oscillation parameters. The bottom row shows the ratio of these two spectra. The $\mathrm{x}$-axis gives the value of $\mathrm{E}_{\mathrm{reco}}$, which is the sum of the muon energy from range and/or curvature plus the energy of the possible hadronic shower. In determining the hadronic shower energy the average detector response per $\mathrm{GeV}$, derived from $\mathrm{MC}$, is used. These plots assume a $\sim 3$-year exposure in the Low Energy beam configuration with $2.5 \times 10^{20}$ protons on target per year.

\subsubsection{Spectral Dependence Measurements}

Accurately measuring the oscillation parameters is best achieved by comparing the $\nu_{\mu} \mathrm{CC}$ energy spectra from the Near and Far detectors, plots of the spectra for three possible values of $\Delta m_{23}^{2}$ are shown in Figure 3.14. The size of the depletion is related to $\sin ^{2}\left(2 \theta_{23}\right)$ and the mean energy of the missing $\nu_{\mu}$ is a measure of $\Delta m_{23}^{2}$. The accuracy with which MINOS should be able to measure the neutrino oscillation parameters is shown in Figure 3.15. The measurement is dependent on both the value of $\Delta m_{23}^{2}$ that nature has chosen and the statistical error, which is driven by the number of protons on target.

Super-Kamiokande's results place the best fit value of $\Delta m_{23}^{2}$ at $2.4 \times 10^{-3} \mathrm{eV}^{2}$. At this value the maximum $\nu_{\mu}$ disappearance occurs around $1.5 \mathrm{GeV}$, which is below the tau production threshold of approximately $4 \mathrm{GeV}$. The low branching ratio for $\tau \rightarrow \mu+\nu_{\mu}$ means 


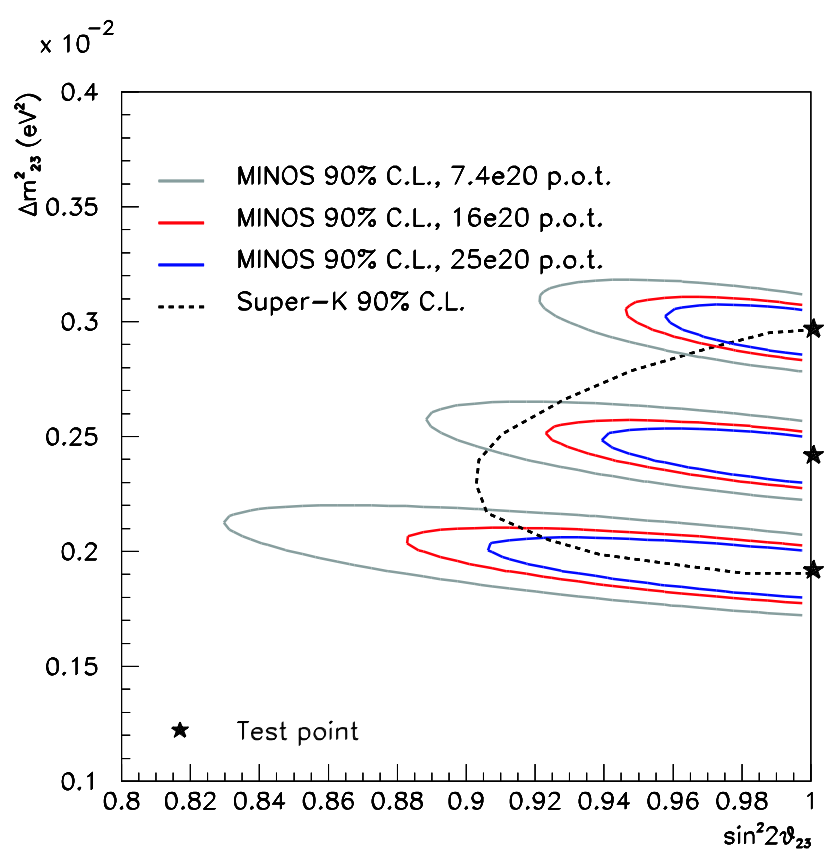

Figure 3.15: Sensitivity of $\nu_{\mu} \rightarrow \nu_{\tau}$ oscillation parameter measurements using spectral dependence measurements (figure is an updated version of one from [81]). The allowed regions at $90 \%$ confidence limit are shown for three different values of $\Delta m^{2}$ and for different numbers of protons on target.

that the small number of taus that are produced will not contribute a significant background to the $\nu_{\mu}$ CC energy spectrum at the Far detector.

A good absolute energy calibration will be necessary to accurately locate the depletion maximum, which determines the value of $\Delta m_{23}^{2}$. Similarly, a good relative calibration is necessary since a shift in the energy scales between the two detectors will affect the comparison of the oscillated and unoscillated spectra. The subject of the energy calibration is investigated in detail in section 7.

It can be seen from Figure 3.14 that the dip in the ratio of the spectra is not as deep as expected. With $\sin ^{2}(2 \theta)=0.9$ the ratio should fall to a value of 0.1 . However, in the plot with $\Delta m^{2}=3.5 \times 10^{-3}$ it can be seen that the dip only falls to a value of 0.2 . A dominant contribution to the smearing of the dip comes from higher energy events with a high multiplicity of final state particles. The coarse nature of the MINOS calorimeter means that, for example, events with 2 pions cannot be reliably separated from events with 3 pions in the final state. For a neutrino of a given energy with a given fraction of the energy going to the hadronic system (the $y$-value) the total kinetic energy of the hadronic system in the 
2 or 3 pion case will be different. If the pions can be identified then their rest masses can be taken into account when calculating the hadronic energy. However, this is not likely to be possible in MINOS and hence smearing of the dip occurs. Further discussion of such effects is given in [82].

\subsubsection{Appearance Searches}

When MINOS was first designed, consideration was given to measurement of $\nu_{\tau}$ appearance. However, the $735 \mathrm{~km}$ baseline of MINOS coupled with the Super-Kamiokande results means that the vast majority of $\nu_{\tau}$ will appear below the $\sim 4 \mathrm{GeV}$ threshold for $\tau$ production. Separation of the $\nu_{\tau}$ from the other neutrino interactions in the MINOS detectors requires a statistical approach, thus such a measurement no longer seems likely.

In recent years the prospect of observing a non-zero value of $\theta_{13}$ has become an important topic since this will determine if observation of CP violation in the leptonic sector is possible. Background rejection of $\mathrm{NC}$ and short CC events as well as $\nu_{e}$ events from the beam is the major challenge of the analysis. The MINOS sensitivity to $\nu_{e}$ appearance and hence $\theta_{13}$ is calculated to be better than the current CHOOZ limit (see Figure 3.16). 


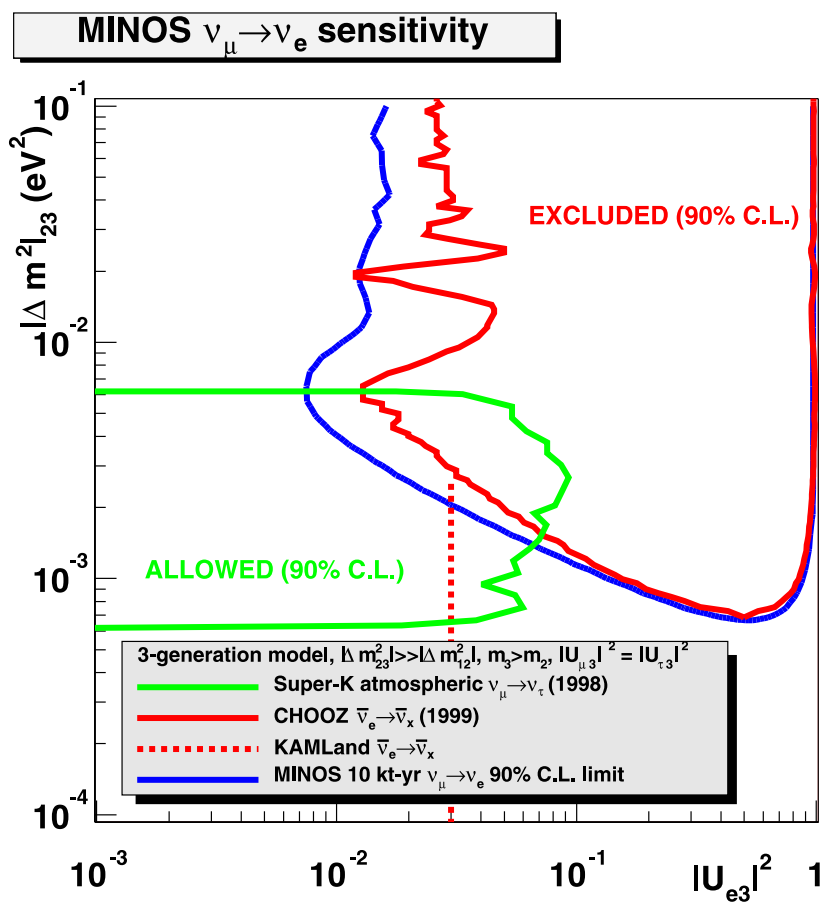

Figure 3.16: Limit on $\nu_{\mu} \rightarrow \nu_{e}$ oscillation parameters (figure from [83]). The $90 \%$ confidence limit, in a three-flavour mixing framework, is shown in blue. Matter effects are included, and the CP-violating phase $\delta$ is assumed to be zero. The exclusion limit from CHOOZ, the projected exclusion limit from KamLAND, and the allowed region from Super-Kamiokande are shown for comparison. 


\section{Chapter 4}

\section{The MINOS Energy Calibration}

To accurately measure the neutrino oscillation parameters it is essential that MINOS have a good energy calibration. However, making precise physics measurements using widely separated, subterranean detectors poses special calibration challenges. For example, there is no controlled source of particles of a known energy and the different experimental environments can introduce systematic errors to the energy scale. This chapter addresses the steps and techniques used to remove the temporal and spatial variations of response within each detector, relate energy deposits from different detectors, and translate the detector response to $\mathrm{GeV}$.

Important tools that MINOS uses for the energy calibration are a light-injection (LI) system and muons. The LI system is a dedicated calibration system that measures the gains, the gain drift over time and the non-linearity of the PMT-electronics combination. Muons are used to normalise both the inter-detector and the intra-detector response. In addition, the Calibration detector used the PS accelerator at CERN as a tool to determine the absolute energy scale for the MINOS detectors. 


\subsection{Energy Calibration Motivation}

The most precise measurements of the oscillation parameters come from determining the neutrino energy spectra at the Near and Far detectors and comparing them, as described in section 3.3.2. Neutrino energy reconstruction of a $\mathrm{CC}$ event first involves determining the energy of both the muon and a possible hadronic shower $E_{\nu}=E_{\mu}+E_{\text {had }}$. The composition of the hadronic shower from a CC neutrino interaction varies considerably and hence so does the energy deposited via ionisation in the detector. A MC event generator is required to simulate this variation in composition of the hadronic showers (see section 4.6).

Muon energy reconstruction is best done by a combination of curvature in the magnetic field and range in the detector. Hence, it is not dependent on knowing each detector's calorimetric response accurately. Although, some calorimetric information may be used to take account of the rare stochastic energy losses that can occur along a muon's track. The three MINOS detectors are similar enough in construction that knowledge of the muon range-to-energy relation can be directly transfered between the detectors to a precision of $<2 \%$.

Shower energy reconstruction is best achieved by calorimetry, which requires accurate knowledge of each detector's response. Calorimetry in the MINOS detectors involves summing up the light output of many scintillator strips for each event. However, there are many factors that vary the response: differing scintillator strip lengths and light yield; temperature fluctuations and other changes over time; non-linearity of PMTs; varying WLS fibre length; different length optical readout cables; reflector connectors in the Near detector; different connector transmission efficiencies; different PMTs and electronics. Calibrating out all these effects is necessary for accurate shower calorimetry.

Normalising the inter-detector and intra-detector calorimetric response is important in the following two ways. Firstly, having the response vary within a single detector, be it temporal or spatial, degrades the detector resolution so more events and hence a longer running time are required for the same measurement accuracy. Secondly, if the events used 
are not evenly distributed across the spatial and/or temporal fluctuations then a systematic bias to the energy scale is introduced.

Systematic bias in the absolute or the relative energy scale will affect the spectral measurements of the neutrinos in the following way. Measurement of $\Delta m_{23}^{2}$ is determined by the position of the dip in the ratio of the $\nu_{\mu} \mathrm{CC}$ energy spectrum measured at the Far detector to the expected spectrum extrapolated from the Near detector measurements and NuMI beam-line simulation (see Figure 3.14). A systematic offset in the absolute energy scale has a direct impact on the measured value of $\Delta m_{23}^{2}$ since the value of $\mathrm{E}$ in equation 2.12 will be wrong. If the relative energy scale at the Near and Far detectors is different then that will affect both the position and the shape of the dip, thus impacting on the measurement of both $\sin ^{2} 2 \theta_{23}$ and $\Delta m_{23}^{2}$. To limit the effect of such systematic errors on the energy scale, calibration targets have been set. MINOS intends to make a $10 \%$ measurement of $\Delta m_{23}^{2}$ and thus aims to reduce the systematic errors to a fraction of this.

In setting the targets it is necessary to consider both what is required and the level of effort necessary. For example, the errors that arise from imprecise knowledge of neutrino interactions and the multiplicity of final state particles are estimated to contribute a several percent error to the measurement of $\Delta m^{2}$. Improving the knowledge of neutrino interactions for MINOS and the next generation of experiments will involve multi-million dollar experiments such as MINERvA [84]. Reducing the error on the absolute energy scale for hadronic interactions from perhaps $15 \%$ to $5 \%$ required running the Calibration detector in the PS test-beams over a period of several years. Such a reduction also contributes an improvement on the precision of the $\Delta m^{2}$ measurement of order a few percent (among other things). Another example involves extrapolating the neutrino beam spectrum to the Far detector: neutrino-beam monitoring equipment has been installed/operated and much effort has been invested on the neutrino-beam MC simulation in order to reduce the error on the extrapolation to $2 \%$. Bearing in mind all the above examples and using knowledge of what is historically achievable with an iron/scintillator detector, an upper limit target of 5\% error on the absolute energy scale has been set. In addition, a tighter upper limit target of $2 \%$ 


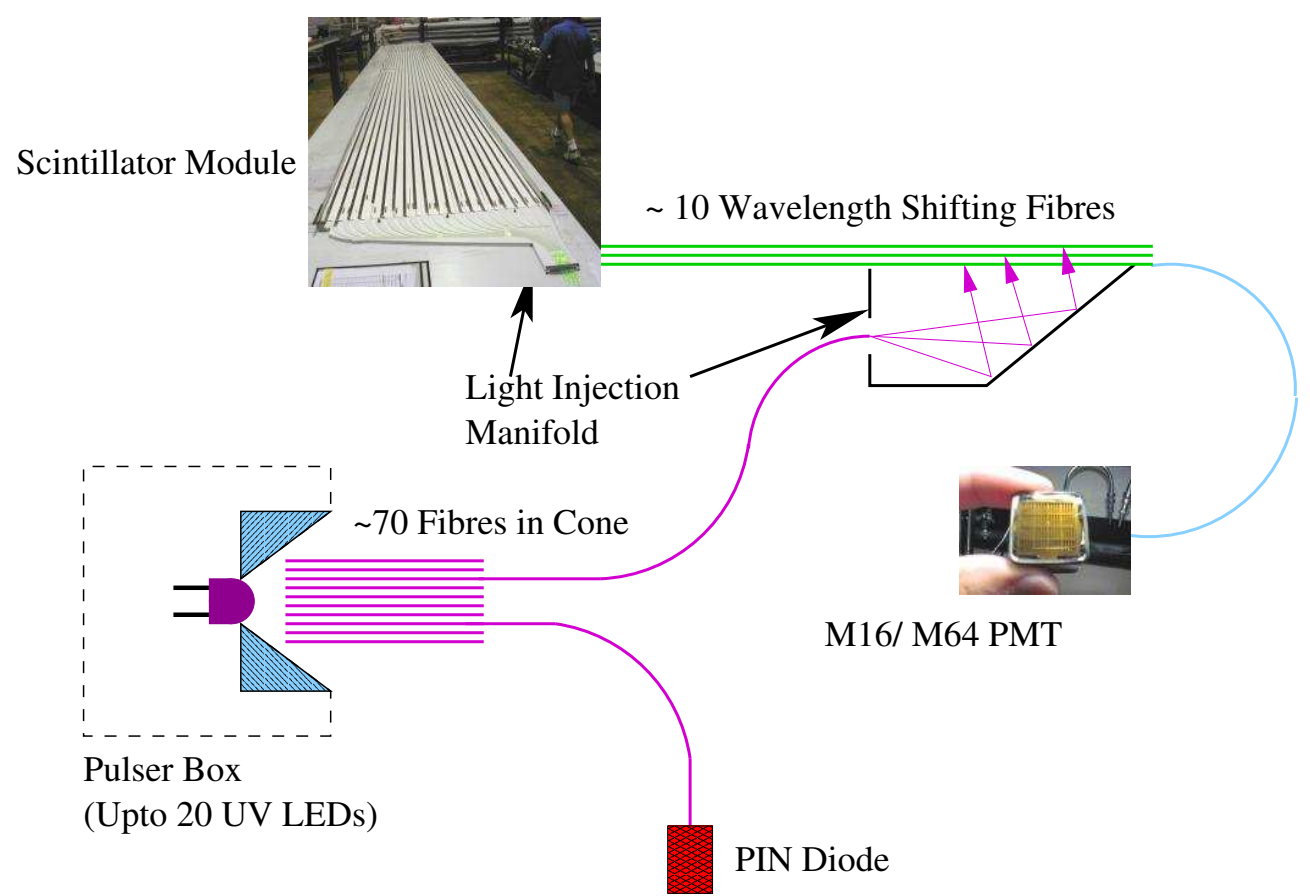

Figure 4.1: A schematic diagram of the light-injection system. Ultra-violet light is generated by LEDs contained in a Pulser Box. The light is routed along fibre optic cables to edge of the scintillator modules where it is injected into the WLS fibres. Once injected into the WLS fibres the light follows the same readout path to the PMT as light created in the scintillator. To provide an independent, stable and linear measure of the quantity of light injected into the standard readout chain PIN photodiodes are used.

error on the relative energy scale has been set since this calibration is believed to require less effort to achieve than reducing the error from some of the other sources would.

\subsection{The Light-Injection System Hardware}

The MINOS light-injection (LI) system is a dedicated, hardware based calibration system [85, 86, 87]. The LI system injects carefully monitored, controlled and variable pulses of light into the wavelength shifting (WLS) fibres to mimic the signals that come from particle energy deposits in the scintillator. A schematic of the LI system is shown in Figure 4.1.

Twenty ultra-violet (UV) LEDs are housed in a custom designed Pulser Box. At the Far detector there are 16 pulser boxes and at the Near detector there are 3 Pulser Boxes. Each LED in a Pulser Box simultaneously illuminates the ends of 71 optical fibres, which are held 
in a reflective aluminium cone. The LI fibres are routed to the edge of the scintillator modules where they enter a light-injection manifold (LIM). Within the LIM the UV light shines on up to 10 WLS fibres just after they have exited the scintillator strips. The absorption and re-emission of the light in the WLS fibre replicates the process within the scintillator strips and the light-path to the PMTs is the same. The duration of each light pulse can be as short as $15 \mathrm{~ns}$ so as to emulate the pulses of light in the WLS fibre coming from the scintillator.

Monitoring of the intensity of the light delivered by each LED is achieved with PIN photodiodes. Since the PINs do not have the sensitivity of the PMTs they require higher levels of light. This is achieved by holding a few fibres from each bundle of 71 closer to the LED. Signals from the PINs are shaped and amplified to resemble those of a PMT. At the Far detector the amplified PIN signals are digitised using an extra channel on the VA chip, at the Near detector dedicated QIE chips are used. The PINs have been shown to have a linear response and to be stable to $0.5 \%$ over a two month period [85].

\subsection{Muons}

The detector response to muon energy deposits is dependent on every stage of the readout chain: the light output of the scintillator; the transmission efficiencies of the optical fibres/connectors; the gain of the PMTs; and the gain of the electronics. This sensitivity to all the detector components makes the muons a valuable calibration tool. In addition, the high penetrating power of muons means that they leave long, easy to recognise tracks; in the energy regime relevant to MINOS $(\sim 0.5-10 \mathrm{GeV})$, muons are well described by the Bethe-Bloch equation and thus they leave well understood energy deposits in the detector; and furthermore, cosmic muons are relatively abundant at all three detectors.

The procedure for using muons is as follows. The path of the muon through the detector is determined by a pattern-recognition/tracking algorithm. Using the track information the muon's path length through each scintillator strip can be determined. Once the path length of the muon is corrected for, the response of the detector is proportional to the muon's 
$\mathrm{dE} / \mathrm{dx}$. For a given flux of cosmic muons the average energy deposited in each strip is fixed thus providing a valuable calibration source. Furthermore, once the muon's energy is known then the Bethe-Bloch equation can be used to calculate the actual energy deposition in GeV.

\subsection{PMT Gain Calibration}

Although the PMT gain calibration is not part of the standard calibration chain it is necessary for the rejection of cross-talk and any calculations that require a zero-correction procedure. Consider first the cross-talk. This feature of the MA PMTs typically occurs because a photo-electron (pe) has fallen into the wrong dynode chain. Such a cross-talk mechanism means that cross-talk most often occurs at the single-pe level. Thus knowing the gain of each channel aids the rejection of cross-talk.

Consider now the need for a zero-correction procedure. The Poissonian nature of PMT signals means that at low light levels it is probable that no photo-electron is generated. Put another way, it means that sometimes when light strikes the PMT face no signal is generated at all. When determining the average response of a scintillator strip it is necessary to include zero responses as well as the occasions when a signal was generated. Including the zeroes can't be done in a direct manner due to zero-suppression (sparsification) in the FEE electronics, but the probability of obtaining zero signal is easily calculable when the gain of the channel is known.

The gain calibration is required in part because MA PMTs are used. With single channel PMTs it is possible to tune the high-voltage setting to obtain a fixed gain. However, MA PMTs only have a single high-voltage setting per PMT. The spread in the gain of the pixels on a single M64 PMT in MINOS is typically between 15-25\% and thus has to be calibrated out.

Various techniques for determining the gain of each PMT pixel have been studied [88]. The most robust technique uses the LI system and a photo-electron counting technique [77]. The Poissonian nature of the PMTs means that the average charge, $\mu$, measured from 
repeated injections of light and the RMS, $\sigma$, of that response are related to the number of photo-electrons generated at the photo-cathode. The gain, g, of the PMT is defined as

$$
g=\frac{\mu}{N_{p e} \times e}
$$

where $N_{p e}$ is the average number of photo-electrons generated per pulse of the light-injection system and $e$ is the charge on an electron. The variance on the average charge is given by

$$
\sigma^{2}=\left(\sqrt{N_{p e}} g e\right)^{2}+\left(\sqrt{N_{p e}} g e w\right)^{2}+\left(\sigma_{p e d}\right)^{2}
$$

where $w$ is the fractional width of the single photo-electron spectrum and $\sigma_{\text {ped }}$ is the RMS of the electronics noise. The width of the single photo-electron spectrum is caused primarily by fluctuations in the number of secondary electrons emitted at the first dynode. When multiple photo-electrons strike the first dynode random fluctuations mean that some produce higher (or lower) numbers of secondary electrons than others, hence the presence of the middle term in equation 4.2 and its dependence on $\sqrt{N_{p e}}$. Combining equations 4.1 and 4.2 gives

$$
N_{p e}=\frac{\mu^{2}}{\sigma^{2}-\sigma_{p e d}^{2}} \times\left(1+w^{2}\right) .
$$

thus the gain is simply

$$
g=\frac{1}{\frac{\mu^{2}}{\sigma^{2}-\sigma_{p e d}^{2}} \times\left(1+w^{2}\right) \times e} .
$$

The values of $w$ have been measured in both pre-installation laboratory tests of the PMTs [89] and in-situ using low light level light-injection runs [77]. The variance of the electronics noise is known from special pedestal runs of the detector. Thus, using equation 4.4, the gain can be straightforwardly calculated using the values of $\mu$ and $\sigma$ obtained from light-injection runs. 


\subsection{The Calibration Chain}

The different calibration steps required to make an accurate calorimetric measurement of a particle's energy deposition are outlined in this section.

\subsubsection{Drift Calibration}

The drift calibration is concerned with correcting for shifts in the response of the detector over time. How these drifts are calibrated out depends on the time-scale over which they occur and the detector component affected. The dominant drifts are caused by temperature fluctuations but other drifts due to, for example, changes in voltage supplies to electronics, aging of the PMTs' photocathode or aging of the scintillator light-output also potentially need correction.

Short-term drifts in the gain of the PMTs that occur on the scale of hours to many weeks are corrected using the LI system. This calibration is achieved by pulsing the LI system $\sim 1000$ times in a few minutes. Taking the ratio of the mean PMT response to the mean PIN response determines the PMT's response to a measured amount of light. The procedure is repeated at regular intervals, thus enabling the gain of the PMTs to be tracked. At the Calibration detector this LI drift correcting procedure was required every 20 minutes, whereas at the Far detector it is only done every 3 hours and at the Near detector it is done every hour.

Drifts in the response of the electronics are harder to correct since the PIN is read out with the same electronics as the PMT and hence the two drift together. Studies, both at a laboratory test stand and in-situ at the Far detector, have shown that the fractional drift of the VA electronics system is $0.0022 \pm 0.0011$ per ${ }^{\circ} \mathrm{C}[90]$. Preliminary studies of the QIE electronics system used at the Near detector have revealed a smaller drift but this effect has not been fully quantified at the time of writing. These relatively small drifts will not have a large effect at the Near or Far detector because the temperature at those locations is generally stable to $1-2{ }^{\circ} \mathrm{C}$. However, at the Calibration detector the temperature fluctuations 
were much larger, around $5{ }^{\circ} \mathrm{C}$. To correct for the drift due to the temperature variations the local air temperature at the Calibration detector is used.

As outlined in section 4.3, measurements of the detector response to muons are sensitive to every stage in the readout chain. Thus, the combined drift of all the components in the readout chain can be measured with muons. However, the time-scale over which these drifts can be measured depends on the muon rate and hence detector location. At the Far detector it takes several months to collect enough cosmic muon events such that the statistical error on the response of each scintillator strip is at the percent level. However, it is possible to determine the average response of the whole Far detector to the $1 \%$ level on the timescale of days. Thus, the cosmic muons provide a valuable cross-check that the LI system is successfully removing the average drift in the gain of the PMTs over periods of several days. Any longer term residual drifts in the measured muon response, once the LI corrections have been applied, provide the final drift calibration. At the time of writing the muons have not yet been used to take out any potential long term drifts in the detectors' response.

\subsubsection{Linearity Calibration}

In order to correctly measure large energy deposits ( $>\sim 100$ photo-electrons) it is necessary to calibrate out the non-linearity of the PMTs and electronics. By injecting light at a range of intensities using the LI system the non-linearity of the combined PMT-plus-electronics can be measured. To achieve an accurate measurement it is necessary to establish a linear reference scale for the light injected. The PIN diodes in the LI system are used to set this linear reference scale since they have been shown to be linear to better than $1 \%$. At the Near detector, the QIE electronics plus PIN-pre-amplifier that read out the PIN diodes are also linear to better than 1\% [91, 92]. The QIE electronics itself is linearised by an online process that uses data from special current injection runs [75]. However, the Far detector VA electronics that reads out the PIN diodes does not have an online linearity calibration and thus an additional step in the offline calibration process is required. 
Far detector electronics non-linearity is measured using a charge-injection (CI) system that is built into the front-end electronics. Special CI runs are taken where charge is injected into individual electronics channels at a range of linearly increasing values and the response is measured. Although it is possible to measure the non-linearity of every Far detector electronics channel, such measurements are only used to linearise the readout of the PINs in the LI system. The non-linearity of the VA electronics channels reading out the PMTs is not corrected for using the CI since the combined non-linearity of the PMT-plus-electronics is measured with the LI system.

\subsubsection{Strip-to-Strip Calibration}

The intra-detector calibration is concerned with normalising the response of each of the scintillator strips within the detector. Through-going cosmic muons are used to perform this calibration [18][38]. Many detector effects are simultaneously removed by this calibration step: light-output of the scintillator; gain of the PMTs and electronics; WLS fibre differences; optical readout fibre length differences; optical connector transmission efficiency; and any other differences between the strips. Once this calibration is performed, the response from the centre of any strip of a particular length is the same. Corrections for the position of a hit along the strip are described in section 4.5.4.

\subsubsection{Fibre Attenuation Calibration}

After the strip-to-strip calibration there is one detector effect remaining to be corrected for: the distance of a hit from the centre of its strip and hence the amount of attenuation in the WLS fibre. This calibration step can only be performed once the position of the hit along its strip is known from full three-dimensional reconstruction. There is a possibility of a circular dependence at this stage, since the reconstruction is not necessarily independent of the calorimetric response. Thus, care has to be taken in the reconstruction process to avoid such a dependence. 
The attenuation in the WLS fibre was measured by scanning a radioactive source along the length of every strip. This mapping procedure was undertaken for each strip in every scintillator module before they were used in the construction of the detectors. The data from each strip are fit to a double-exponential function and used in the calibration.

The mapping procedure was an important quality assurance process, which allowed poorly constructed modules to be rejected. The variation in attenuation along the length of a strip relative to the average strip is comparatively small, with a rms of $\sim 4 \%$ at the end of a strip. However, removing the variation on a strip-by-strip basis improves the energy resolution and reduces the possibility of systematic effects. Note that it is only the differences in attenuation that are relatively small, the attenuation calibration itself is very significant with the response varying by a factor of $\sim 5$ between the two ends of a $8 \mathrm{~m}$ strip.

It is possible to use muons to measure the attenuation along each strip but large statistical samples are required. At the Far detector, such a calibration would require all the cosmic muon events collected over a period of several years. However, at the Calibration detector enough cosmic muons are collected over a period of days.

Using the position of each hit along its strip and the double-exponential function for that strip the calibration can be performed.

\subsubsection{Inter-detector Calibration}

The final stage in the calibration chain, before a conversion to absolute energy deposition in $\mathrm{GeV}$, is the inter-detector calibration. This calibration step serves to normalise the response of a plane of scintillator in all three detectors to each other. Muons are used for this task because they are abundant enough at all three detectors and their momentum can be measured by independent, non-calorimetric means.

If the cosmic muon energy spectrum were the same at each detector then the average response of all the muons could be used to do the relative calibration. However, the different locations of the detectors means that the average cosmic muon energy differs considerably: 
at the Calibration detector the average energy is approximately $4 \mathrm{GeV}$ whereas at the Far detector, $710 \mathrm{~m}$ underground, the average energy is of order $100 \mathrm{GeV}$. Consider muons traversing through iron: at $4 \mathrm{GeV} d E / d x=1.816 \frac{\mathrm{MeV}}{\mathrm{g} / \mathrm{cm}^{2}}$ whereas at $100 \mathrm{GeV} d E / d x=$ $2.773 \frac{\mathrm{MeV}}{\mathrm{g} / \mathrm{cm}^{2}}$, which is $53 \%$ greater. Any correction to the measured scintillator response would be dependent on both knowing the muon spectrum at all three detectors accurately and on the $\mathrm{dE} / \mathrm{dx}$ calculations. Thus, achieving the $2 \%$ relative calibration target using an average of all the cosmic muons would be extremely difficult.

To accurately determine the energy scale of each detector, the momentum of each muon used in the calibration has to be known. MINOS has two methods for measuring muon momentum: for muons that stop in the detector, range is the best measure; for muons that are through-going, curvature in the magnetic field is used.

As outlined in chapter 1, the focus of this thesis is on using stopping muons to perform the inter-detector calibration (see chapter 5 for a detailed description). Since a stopping muon loses all its energy in the detector it is straightforward to determine what the energy of the muon was at various points along its track. In fact, because the calibration is relative it is sufficient to only know the amount of material traversed by the muon in each detector. The similarity of the detectors' construction ensures that the muon's energy loss, as it comes to a stop, is the same ${ }^{1}$ in each detector.

After the inter-detector calibration a particle, of given energy and type, passing through a plane of scintillator in any of the three detectors gives an identical response (to within the errors of the calibration). However, it is important to note that the absolute quantity of energy deposited in one full, steel-scintillator plane depends on both the thickness of the steel and to second order the thickness of the scintillator. In other words, an identical calorimetric response from a given plane in the different detectors does not necessarily correspond to an exactly identical energy deposition.

The steel used in the Calibration detector is $1.6 \%$ thinner $(2.5 \mathrm{~cm}$ instead of $2.54 \mathrm{~cm})$ than

\footnotetext{
${ }^{1}$ Small corrections, at the $\sim 1 \%$ level, have to be made to account for the different steel thicknesses (see section 3.2) both within a detector and between detectors.
} 
the steel in the Near or Far detectors. Thus, the energy deposition per plane by a particle of given energy will be $1.6 \%$ higher in the Near or Far detector than in the Calibration detector. It is also necessary to correct for any differences in steel thickness within each detector. For example, the first 190 planes of the Far detector are approximately 1\% thicker than the other 296 planes [66]. Thus, when calculating a precise energy deposition in the first 190 planes of the Far detector a correction for steel thickness has to be made.

\subsubsection{Absolute Energy Calibration}

The absolute energy calibration requires a suitable source of electrons and hadrons of known energy, which does not exist on-site at the Near and Far detectors. Many experiments have used the Michel electrons from muon decay or by reconstructing the invariant mass of a $\pi^{0}$ in setting their energy scale but this is not practical in the MINOS detectors. There are a number of reasons for this; the steel planes are 1.4 radiation lengths thick and the scintillator strips $4.1 \mathrm{~cm}$ wide thus the granularity is too poor for reliably identifying $\pi^{0}$ events; and the lifetime of a muon is $2.2 \mu \mathrm{s}$, which is long relative to the live time of the Far detector electronics (the VA chip has an integration and shaping time of $\sim 500 \mathrm{~ns}$ after which it is dead for $\sim 5 \mu \mathrm{s})$.

Exposing the MINOS Calibration detector to particle beams of known energy at the CERN PS accelerator meant that the absolute scale of the detector response was determined [68][70][69]. Results obtained from the Calibration detector are used to tune the single-particle response in the MC. Furthermore, the MC is tuned so that the relative response of different particle types is correct, e.g. electrons and pions, or electrons and muons.

Calibration of the absolute scintillator response to an energy deposition in $\mathrm{GeV}$ can also be achieved using the relative calorimetric energy measurements from stopping muons and the MC simulation. Knowing the momentum of the stopping muons allows calculation of the $\mathrm{dE} / \mathrm{dx}$. Combining the calculated $\mathrm{dE} / \mathrm{dx}$ with the known scintillator thickness determines the absolute energy deposition per plane of scintillator in GeV. This calculated value of absolute energy deposition in $\mathrm{GeV}$ per scintillator strip gives the conversion factor between 
the measured detector response and $\mathrm{GeV}$. It is important to note that this conversion factor only allows calculation of the energy deposition in the scintillator, the energy deposition in the steel will be different.

\subsection{Neutrino Energy Calculation}

Reconstruction of the initial neutrino energy involved in, say, a CC muon neutrino interaction is the sum of the visible plus invisible energy. The visible energy comprises the muon energy and a hadronic shower, the invisible energy occurs for reasons such as pion reabsorption. As has already been discussed, muon momentum is relatively easily reconstructed by range and/or curvature in the magnetic field. In contrast, determining the hadronic shower energy plus invisible energy is relatively complex.

The difficulties in determining the invisible energy in the average, say, $\nu_{\mu} \mathrm{CC}$ event occur for a number of reasons [82]: the multiplicity of final state particles and their masses has an impact on the invisible energy in the few $\mathrm{GeV}$ regime; pion reabsorption in the nucleus and nuclear re-scattering also affect the amount of invisible energy as a function of energy. A MC event generator is required to take account of all these effects. In MINOS the NEUGEN [93] event generator is used to estimate the fraction of visible and invisible energy for each class of event, e.g. DIS, resonance or quasi-elastic and for all the different neutrino energies. Currently the available information about neutrino interactions at the energies of interest to MINOS is limited and consequently the predictions of the event generator have some significant errors attached to them. The MINERvA experiment aims to substantially improve the knowledge of neutrino interactions by running in the NuMI beamline [84].

Even once the visible energy of the interaction is known, the quantity of energy deposited in the scintillator varies significantly. The type and quantity of particles produced in the hadronic shower, or "shower fractions", change the total energy deposition in the scintillator. Depending on the fractions of, for example $p^{+}, \pi^{ \pm}, \pi^{0}$, the energy deposited in the scintillator for a given quantity of visible shower energy varies significantly. Exactly what the energy 
deposition in the scintillator is, given the different shower fractions, is estimated using the GEANT MC [94].

Once the absolute energy calibration has been achieved (as set out in section 4.5.6) the NEUGEN event generator and GEANT MC can be used to estimate the response (in GeVs) of the MINOS detectors to hadronic and electromagnetic showers produced in neutrino interactions. 


\section{Chapter 5}

\section{Relative Energy Calibration Technique Using Stopping Muons}

Stopping muons are a "standard candle" for MINOS that allow the relative calorimetric energy response of each detector to be accurately measured. The relative energy calibration is performed by normalising the measured response of the three detectors. Stopping muons are used because they are the type of particle that have the most accurately known energy at all three MINOS detectors.

The focus of this chapter is on describing the physics relevant to stopping muons in the MINOS detectors as well as the details of the techniques and algorithms used in the calibration (details of the stopping muon selection in each detector are given in the next chapter). Specifically, a "track window" technique that allows precise measurement of each detectors' relative calorimetric response is introduced and described in this chapter. 


\subsection{Muons in MINOS}

\subsubsection{The Bethe-Bloch Equation}

The ionisation energy loss of stopping muons in the MINOS detectors is well described by the Bethe-Bloch equation [23]

$$
-\frac{d E}{d x}=K z^{2} \frac{Z}{A} \frac{1}{\beta^{2}}\left[\frac{1}{2} \ln \frac{2 m_{e} c^{2} \beta^{2} \gamma^{2} T_{\max }}{I^{2}}-\beta^{2}-\frac{\delta}{2}\right]
$$

where $z e, \beta c$ and $\gamma$ are the charge, velocity and relativistic gamma factor of the incident particle. $T_{\max }$ is the maximum kinetic energy which can be imparted to a free electron in a single collision. $Z / A, I$ and $\delta$ are the ratio of the charge number to the mass number, mean excitation energy and density effect correction for the material traversed by the incident particle. The constant, $K=4 \pi N_{A} r_{e}^{2} m_{e} c^{2}$, involves Avagadro's number, $N_{A}$, the classical electron radius, $r_{e}$, and the rest energy of the electron, $m_{e} c^{2}$.

Figure 5.1 shows the stopping power vs. momentum for $\mu^{+}$in $\operatorname{copper}^{1}$. The region between a muon momentum of approximately $0.005 \rightarrow 50 \mathrm{GeV} / \mathrm{c}$ (shown by the vertical grey bars) is well described by the Bethe-Bloch equation. The energy loss described by the Bethe-Bloch equation is due to ionisation of the medium being traversed. The physical process involved in the ionisation is simply electromagnetic scattering of the incident particle with the atomic electrons. There are some key features of the Bethe-Bloch curve that are relevant to stopping muons in the MINOS detectors. A minimum occurs at $\beta \simeq 0.96$, or $p_{\mu} \simeq 0.35 \mathrm{GeV} / \mathrm{c}$. On the left-hand side of the minimum the stopping power increases rapidly as $\sim \beta^{-2}$ whereas on the right-hand side the stopping power increases as $\ln (\beta \gamma)$. The slow logarithmic increase in stopping power is refered to as the "relativistic rise". Part of the relativistic rise comes from the increase in $T_{\max }$ that comes with increasing momentum.

\footnotetext{
${ }^{1}$ The stopping power in copper is very similar to that in iron. When a muon is approximately minimum ionising $(\sim 360 \mathrm{MeV} / \mathrm{c})$ the stopping power is $\sim 3 \%$ lower in copper than in iron. Whereas, at very high energies $(100 \mathrm{TeV} / \mathrm{c})$ the slightly higher $\mathrm{z}$ in copper means that the stopping power is $\sim 7 \%$ higher in copper than in iron. At around $120 \mathrm{GeV} / \mathrm{c}$ the stopping power in iron and copper is equal.
} 


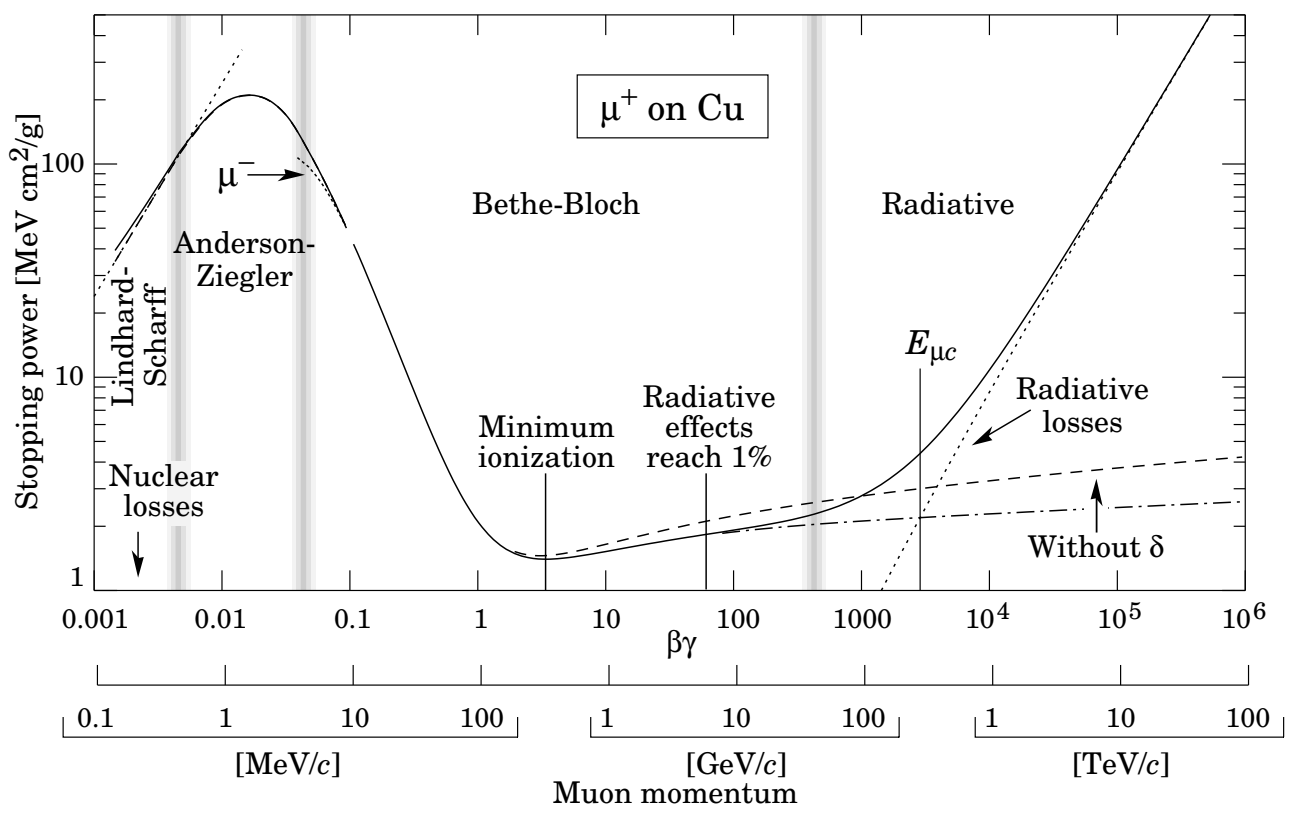

Figure 5.1: Stopping power for positive muons in copper as a function of momentum. The region of interest for MINOS is between approximately $10 \mathrm{MeV}$ to a few $100 \mathrm{GeV}$. In this region the stopping power in copper is very similar $( \pm 3 \%)$ to that in iron. Plot taken from the Particle Data Book [23].

$T_{\max }$ is given by

$$
T_{\max }=\frac{2 m_{e} c^{2} \beta^{2} \gamma^{2}}{1+2 \gamma m_{e} / M+\left(m_{e} / M\right)^{2}}
$$

where $M$ is the mass of the incident particle and the other variables are defined as for equation 5.1. It can be seen that $T_{\max }$ increases as $\sim \gamma^{2}$ for moderately relativistic velocities. The consequence of $T_{\max }$ increasing rapidly is that for a muon of, say, $2 \mathrm{GeV}$ a large fraction $(\sim 20 \%)$ of its energy can be transfered to an electron in a single collision. The electrons receiving large energy transfers are called delta rays. These delta rays can travel significant distances away from the trajectory of the incident particle. For example, in the MINOS detectors it is possible for a delta ray created by a stopping muon to penetrate through a few planes. The presence of delta rays means that the energy lost in a given plane of scintillator is not necessarily the same as the energy deposited.

Ionisation is by far the dominant energy loss mechanism for stopping muons in the MINOS detectors since the muons are well below their critical energy ${ }^{2}$ of $347 \mathrm{GeV}$ in iron and $1.18 \mathrm{TeV}$

\footnotetext{
${ }^{2}$ The definition of the critical energy used by Groom [95] is: the particle energy at which the energy loss by ionisation is exactly half the total energy loss.
} 


$\begin{array}{cccccc}\begin{array}{c}\text { P } \\ (G e V / c)\end{array} & \begin{array}{c}\text { Ionisation } \\ \left(\frac{\mathrm{MeV}}{\mathrm{g} / \mathrm{cm}^{2}}\right)\end{array} & \begin{array}{c}\text { Brems- } \\ \text { strahlung } \\ \left(\frac{\mathrm{MeV}}{\mathrm{g} / \mathrm{cm}^{2}}\right)\end{array} & \begin{array}{c}\text { Pair } \\ \text { production } \\ \left(\frac{\mathrm{MeV}}{\mathrm{g} / \mathrm{cm}^{2}}\right)\end{array} & \begin{array}{c}\text { Photo- } \\ \text { nuclear } \\ \left(\frac{\mathrm{MeV}}{\mathrm{g} / \mathrm{cm}^{2}}\right)\end{array} & \begin{array}{c}\text { Fraction } \\ \text { non-ionising }\end{array} \\ & & 0.001 & & 0.000 & 0.001 \\ 1 & 1.581 & 0.004 & 0.003 & 0.002 & 0.005 \\ 10 & 1.806 & 0.014 & 0.014 & 0.004 & 0.016 \\ 20 & 1.942 & 0.033 & 0.038 & 0.008 & 0.039\end{array}$

Table 5.1: Contribution of different muon energy loss mechanisms in iron. The energy loss (in $\frac{\mathrm{MeV}}{\mathrm{g} / \mathrm{cm}^{2}}$ ) due to ionisation, bremsstrahlung, direct pair production and photonuclear interaction at different energies are detailed. The fraction of energy lost by non-ionisation mechanisms at each energy is also listed. Data taken from the Groom tables [95].

\begin{tabular}{|c|c|c|c|c|c|}
\hline $\begin{array}{c}\mathbf{P} \\
(G e V / c)\end{array}$ & $\begin{array}{l}\text { Ionisation } \\
\quad\left(\frac{\mathrm{MeV}}{\mathrm{g} / \mathrm{cm}^{2}}\right)\end{array}$ & $\begin{array}{c}\text { Brems- } \\
\text { strahlung } \\
\left(\frac{\mathrm{MeV}}{\mathrm{g} / \mathrm{cm}^{2}}\right)\end{array}$ & $\begin{array}{c}\text { Pair } \\
\text { production } \\
\left(\frac{\mathrm{MeV}}{\mathrm{g} / \mathrm{cm}^{2}}\right)\end{array}$ & $\begin{array}{l}\text { Photo- } \\
\text { nuclear } \\
\left(\frac{\mathrm{MeV}}{\mathrm{g} / \mathrm{cm}^{2}}\right)\end{array}$ & $\begin{array}{c}\text { Fraction } \\
\text { non-ionising }\end{array}$ \\
\hline 1 & 2.048 & 0.000 & & 0.000 & 0.000 \\
\hline 4 & 2.275 & 0.001 & 0.001 & 0.002 & 0.002 \\
\hline 10 & 2.414 & 0.004 & 0.004 & 0.005 & 0.005 \\
\hline 20 & 2.509 & 0.009 & 0.010 & 0.009 & 0.011 \\
\hline
\end{tabular}

Table 5.2: Contribution of different muon energy loss mechanisms in polystyrene. The energy loss (in $\frac{\mathrm{MeV}}{\mathrm{g} / \mathrm{cm}^{2}}$ ) due to ionisation, bremsstrahlung, direct pair production and photonuclear interaction at different energies are detailed. The fraction of energy lost by non-ionisation mechanisms at each energy is also listed. Data taken from the Groom tables [95].

in polystyrene. Table 5.1 and 5.2 detail the energy loss by ionisation, bremsstrahlung, direct pair production and photonuclear interaction at different energies in iron and polystyrene respectively. The fraction of energy lost by non-ionisation mechanisms in iron (polystyrene) at $1 \mathrm{GeV}$ is $0.1 \%(0.0 \%)$ but by $20 \mathrm{GeV}$ it has risen to approximately $4 \%(1 \%)$.

\subsubsection{Muon Energy Loss in the MINOS Detectors}

Energy loss in the MINOS detectors is dominated by that occurring in the steel. Stopping muons lose approximately $30 \mathrm{MeV}$ in each plane of steel, except right at the end of the track where the muon comes to rest and the ionisation increases rapidly. The lower density and thinner sheets of polystyrene scintillator only absorb approximately $2 \mathrm{MeV}$ per plane, which is only a 15 th of the energy lost in the steel. Figure 5.2 shows the calculated energy losses 

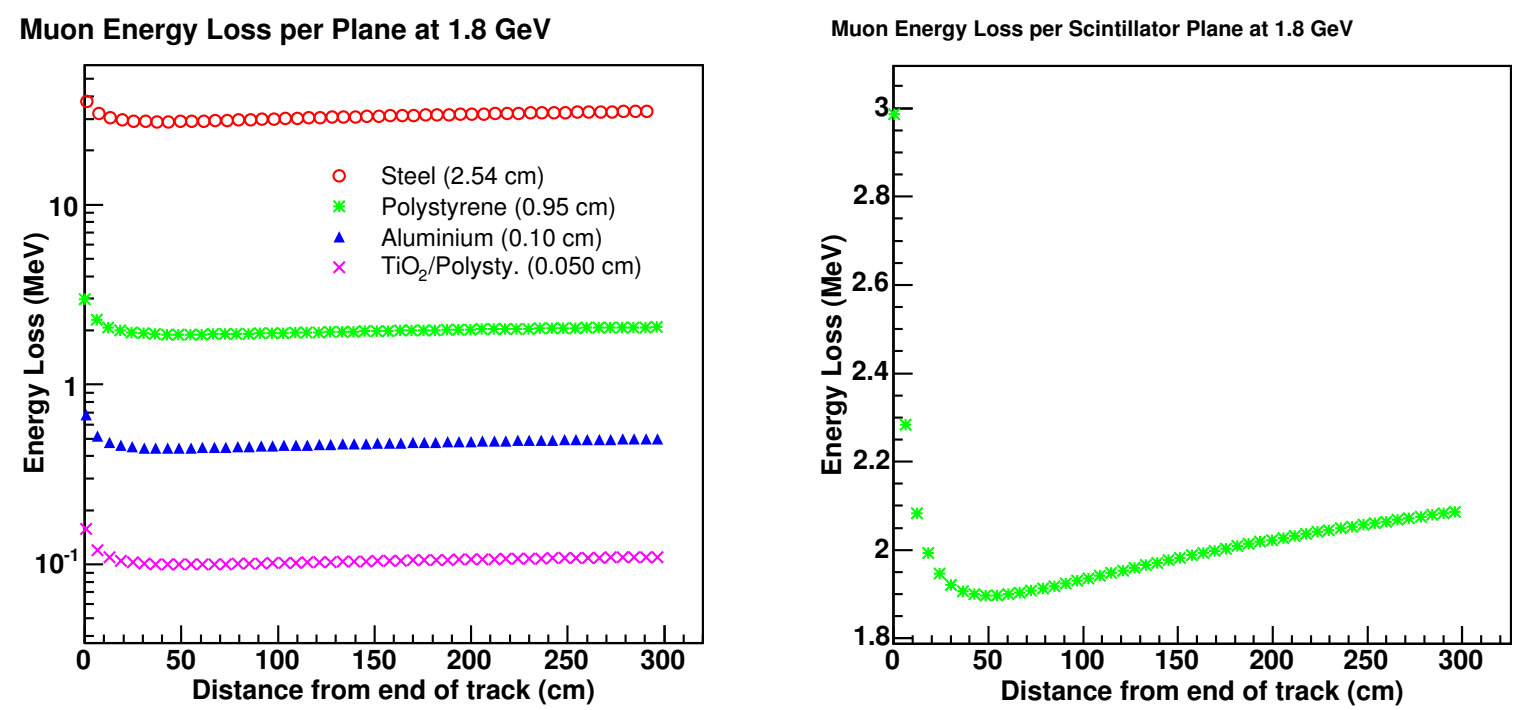

Figure 5.2: Calculated muon energy loss per plane. The energy loss as a function of distance from the end of a stopping muon track is shown. The left plot shows the contribution to the energy loss from the different components of a plane (steel, polystyrene scintillator, aluminium scintillator module casing and $\mathrm{TiO}_{2}$-polystyrene composite strip coating). The right plot shows, on a linear scale, the energy loss in the polystyrene scintillator. The sharp rise in $\mathrm{dE} / \mathrm{dx}$ near the end of the track can be clearly seen.

for a $1.8 \mathrm{GeV}$ muon as it traverses approximately $3 \mathrm{~m}$ through a MINOS detector before coming to a stop. Smaller energy losses occur in the aluminium cases of each scintillator module, amounting to approximately $1 / 6$ th of that in the scintillator itself. Approximately $0.1 \mathrm{MeV}$ per plane is lost in the $\mathrm{TiO}_{2}$-polystyrene composite layer coating each strip since it is only $1 / 20$ th the thickness of the active polystyrene scintillator. Muon energy loss in the air gap between steel sheets is $<10 \mathrm{keV}$ and thus negligible. Table 5.3 lists the energy losses for a minimum ionising particle in the various materials that comprise a plane.

At around $45 \mathrm{~cm}$ from the end of the stopping muon track the energy loss per plane is at a minimum, as can be seen in Figure 5.2. The difference in gradient between the right and left sides of the minimum is substantial. In the region $45-300 \mathrm{~cm}$ from the end of the track the energy loss per plane increases by only $15 \%$, yet between $0-45 \mathrm{~cm}$ from the end of the track the energy loss per plane changes by at least $50 \%$. 


$\begin{array}{lccc}\text { Material } & \begin{array}{c}\text { Thickness Per } \\ \text { Plane }(\mathrm{cm})\end{array} & \begin{array}{c}\text { Energy Loss } \\ (\mathrm{MeV})\end{array} & \begin{array}{c}\text { Fraction of } \\ \text { Loss }\end{array} \\ \text { Steel } & 2.54 & 28.9 & 0.922 \\ \text { Polystyrene } & 0.95 & 1.90 & 0.061 \\ \text { Aluminium } & 0.10 & 0.44 & 0.014 \\ \mathrm{TiO}_{2} \text {-polystyrene } & 0.05 & 0.10 & 0.003 \\ \text { Air } & 2.30 & 0.01 & 0.000 \\ \text { Plane total } & 5.94 & 31.3 & 1.0\end{array}$

Table 5.3: Calculated minimum ionising particle energy loss per plane. The contribution to the energy loss from the different materials (steel, polystyrene scintillator, aluminium scintillator module casing, $\mathrm{TiO}_{2}$-polystyrene composite strip coating and air) that make up a plane are detailed. Note that at the Calibration detector the steel thickness is $2.50 \mathrm{~cm}$ and thus the energy loss per plane and the fraction of loss have to be adjusted by $\sim 1.5 \%$.

$\begin{array}{ccc}\mathbf{P} & \theta_{0} \text { for } \mathbf{2 . 5 4} \mathbf{~ c m} & \theta_{0} \text { for } \mathbf{5} \times \mathbf{2 . 5 4} \mathbf{~ c m} \\ (G e V / c) & \text { steel } & \text { steel } \\ 1.0 & 0.9^{\circ} & 2.2^{\circ} \\ 0.5 & 1.9^{\circ} & 4.5^{\circ} \\ 0.3 & 3.1^{\circ} & 7.5^{\circ} \\ 0.2 & 4.6^{\circ} & 11.2^{\circ}\end{array}$

Table 5.4: Muon multiple scattering in steel. The width of the angular distribution $\left(\theta_{0}\right)$ of the scattered muon, as given by equation 5.3, is listed for a range of energies and for two different thicknesses of steel.

\subsubsection{Muon Multiple Scattering}

The multiple scattering of a muon increases rapidly as it comes to a stop. This process is described by Molière's theory [23]. The width of the angular distribution of the scattered particle is given by

$$
\theta_{0}=\frac{13.6 \mathrm{MeV}}{\beta c p} z \sqrt{x / X_{0}}\left[1+0.038 \ln \left(x / X_{0}\right)\right]
$$

where $p, \beta c$ and $z$ are the momentum, velocity, and charge number of the incident particle, and $x / X_{0}$ is the thickness of the scattering medium in radiation lengths. Table 5.4 lists the value of equation 5.3 for muons of different energies passing through two different thicknesses of steel. 


$\begin{array}{cc}\mathbf{P}(\mathrm{GeV} / \mathrm{c}) & \text { Radius of curvature }(\mathrm{m}) \\ 1.0 & 3.5 \\ 0.5 & 1.8 \\ 0.3 & 1.1 \\ 0.2 & 0.7\end{array}$

Table 5.5: Muon radius of curvature in a $1 \mathrm{~T}$ magnetic field. The radius of curvature has an approximately linear dependence on the energy of the muon in the energy range listed.

\subsubsection{Muon Curvature in the Magnetic Field}

As described in chapter 3, the Near and the Far detectors are magnetised and have a field of between 1-1.5 $\mathrm{T}$ in the fiducial volume. The Calibration detector is not magnetised.

The force on a particle with charge $q$, travelling at velocity $\vec{v}$ in a magnetic field $\vec{B}$ is given by

$$
\vec{F}=q \vec{v} \times \vec{B} .
$$

Equating equation 5.4 with the centripetal force gives the particle's radius of curvature

$$
r=\frac{\gamma m_{0} v}{q B},
$$

where $\gamma$ is the relativistic $\gamma$-factor and $m_{0}$ is the rest mass of the particle. Table 5.5 lists the radius of curvature for a muon in a $1 \mathrm{~T}$ field at various different energies. The radius of curvature has an approximately linear dependence on energy for a relativistic muon.

\subsection{Relative Energy Calibration Technique}

To use muons for the relative energy calibration it is necessary to know their energy since the $\mathrm{dE} / \mathrm{dx}$ varies as a function of momentum. The similarity in construction of the MINOS detectors means that stopping muons of a given track length will, to reasonable approximation, deposit the same amount of energy in all three detectors. Thus, measurement of all three detectors' response to stopping muons of a given track length provides a simple technique of performing the inter-detector calibration. However, determination of a muon's 
energy via a range measurement is expected to have a relative accuracy of only $2 \%$ between the MINOS detectors. This uncertainty comes from various sources, such as steel thickness, curvature in the magnetic field of the Near and Far detectors and the hardware trigger used in the front-end electronics at the Far detector. Given that the relative calibration has to be accurate to better than $2 \%$ this dependence on the stopping muon range measurement is not acceptable.

\subsubsection{Track Window Technique}

The track window technique provides a way of performing the relative energy calibration that minimises the dependence on the accuracy of the stopping muon range (and hence energy) reconstruction between the three detectors.

In order to minimise the dependence on the muon energy measurement between detectors it is necessary to consider how the $\mathrm{dE} / \mathrm{dx}$ varies with momentum. The $\mathrm{dE} / \mathrm{dx}$ of a $1.5 \mathrm{GeV}$ muon increases by approximately a factor of two in the last $10 \%$ of its track, whereas in the other $90 \%$ of its track the $\mathrm{dE} / \mathrm{dx}$ only changes by approximately $8 \%$. The track window technique exploits the portion of the muon track where the $\mathrm{dE} / \mathrm{dx}$ is varying slowly. This technique measures the response of muons only when their energy is between approximately 0.5-1.1 GeV. The section of the muon track when the energy is between 0.0-0.5 GeV is discarded. By only using the track section between $0.5-1.1 \mathrm{GeV}$ the rapid increase in ionisation at the end of the track is avoided (see Figure 5.1). Since the $\mathrm{dE} / \mathrm{dx}$ varies so slowly in the $0.5-1.1 \mathrm{GeV}$ region, a $2 \%$ error on knowing where the muon stopped means only an approximately $0.2 \%$ error in the energy deposition.

\section{Track Window Parameters}

The size of track window used to do the calibration presented in this thesis is $83 \mathrm{~cm}$ wide (or 14 planes traversed perpendicularly, i.e. $14 \times 5.94=83 \mathrm{~cm}$ of material) and the window is positioned $95 \mathrm{~cm}$ (or 16 planes traversed perpendicularly, i.e. $16 \times 5.94=95 \mathrm{~cm}$ of material) 


\section{Track Window Position}

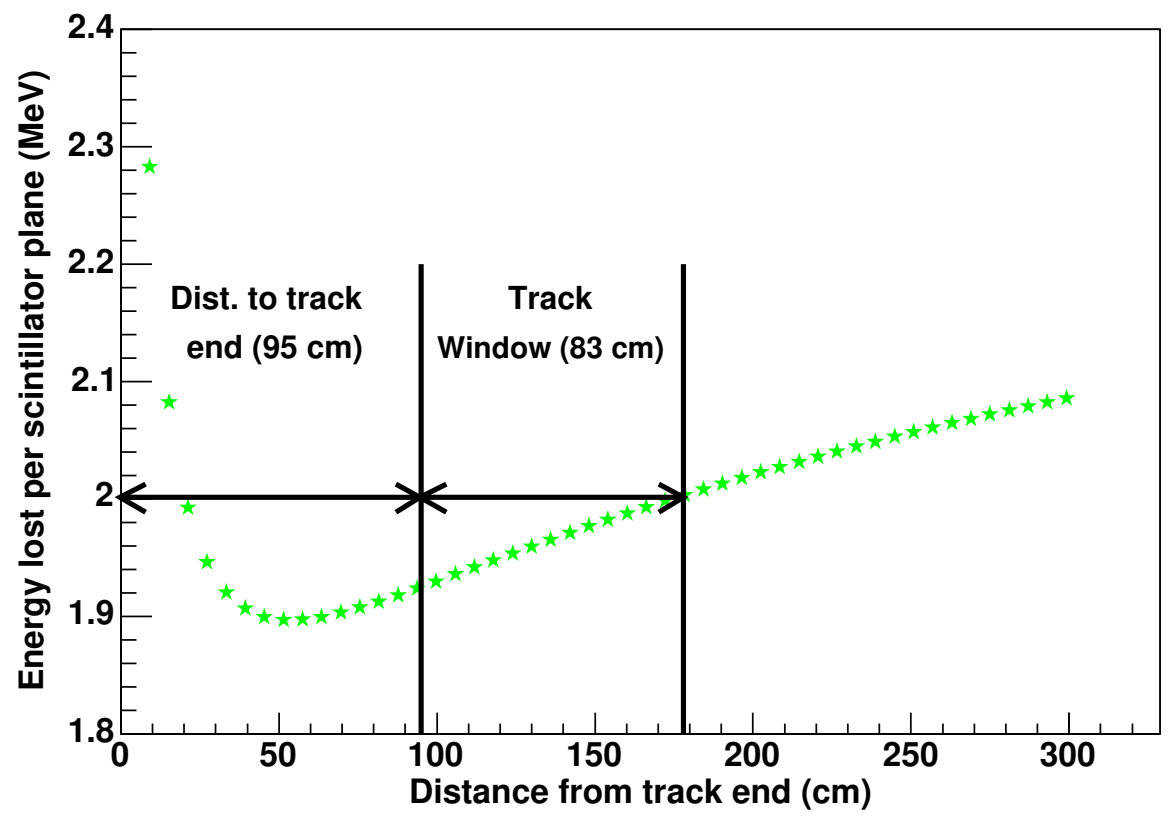

Figure 5.3: Track window position. The track window position is shown relative to the muon energy loss as a function of distance from the end of the track.

from where the muon stopped. Figure 5.3 shows how the energy loss of a stopping muon varies as a function of distance from the end of the track; the position and size of the track window relative to the energy loss is shown.

In order to strictly and rigorously define the position and size of the track window it is necessary to consider what is the most important aspect of the relative calibration. The aim of the relative calibration is to normalise the response of a plane of scintillator in each detector (in data and MC). Thus, the important point is not that the window is $83 \mathrm{~cm}$ wide but that it is exactly 14 planes wide (for a perpendicularly travelling muon). The use of units of $\mathrm{cm}$ is to facilitate the explanation of the procedure when the muons are not travelling perpendicularly. So, if, say, some of the planes in a detector were slightly closer together than the nominal $5.94 \mathrm{~cm}$ there would be no need to correct for it when calculating the size and position of the track window. 


\section{Optimising the Track Window Position}

In optimising the position of the track window there are a number of constraints to be satisfied. The two main parameters that require optimisation in the track window technique are the size of the window and the distance between where the muon stopped and the start of the window. One of the most stringent constraints is the relatively small size of the Calibration detector; it is 60 planes long, which is only enough to contain a $2.0 \mathrm{GeV}$ muon travelling perpendicularly. However, in order to use $1.4 \mathrm{GeV}$ muons at the Calibration detector the sum of the size of the window plus the distance to the end of the track must not exceed 42 planes $(42 \times 5.94 \simeq 250 \mathrm{~cm})$.

The primary constraint in the positioning of the track window comes from the need to avoid the region where the $\mathrm{dE} / \mathrm{dx}$ is changing rapidly with the energy of the muon. A simplistic simulation of muon energy loss is used to evaluate the optimal position of the window. The simulation uses the Bethe-Bloch formula (implemented in the MuELoss software package [96]) and incorporates the necessary information about the geometry of a MINOS plane. Muons are propagated through consecutive planes until their energy loss in a plane would be greater than their remaining kinetic energy. To simplify matters, additional effects such as multiple scattering or curvature in the magnetic field are neglected and it is assumed the muons are perpendicular to each plane.

Studies of muon energy deposition in track windows of various sizes and positions have been made using the simple $\mathrm{dE} / \mathrm{dx}$ simulation. Figure 5.4 shows how the normalised energy deposition within the track window varies with the starting position of the window. It can be seen that when the window is chosen to start close to where the muon stopped the energy deposited in the window changes rapidly. In this case, a systematic mis-reconstruction of the track end point in one of the three detectors by 1 plane would change the quantity of energy deposited in the window by as much as $4 \%$, which is well above the relative calibration target of $2 \%$. However, moving the window away from where the muon stopped by say 10 planes minimises the dependence on the track range reconstruction. In this case, a systematic mis-reconstruction of 1 plane gives only a $0.3 \%$ shift of the energy deposited in the window, 


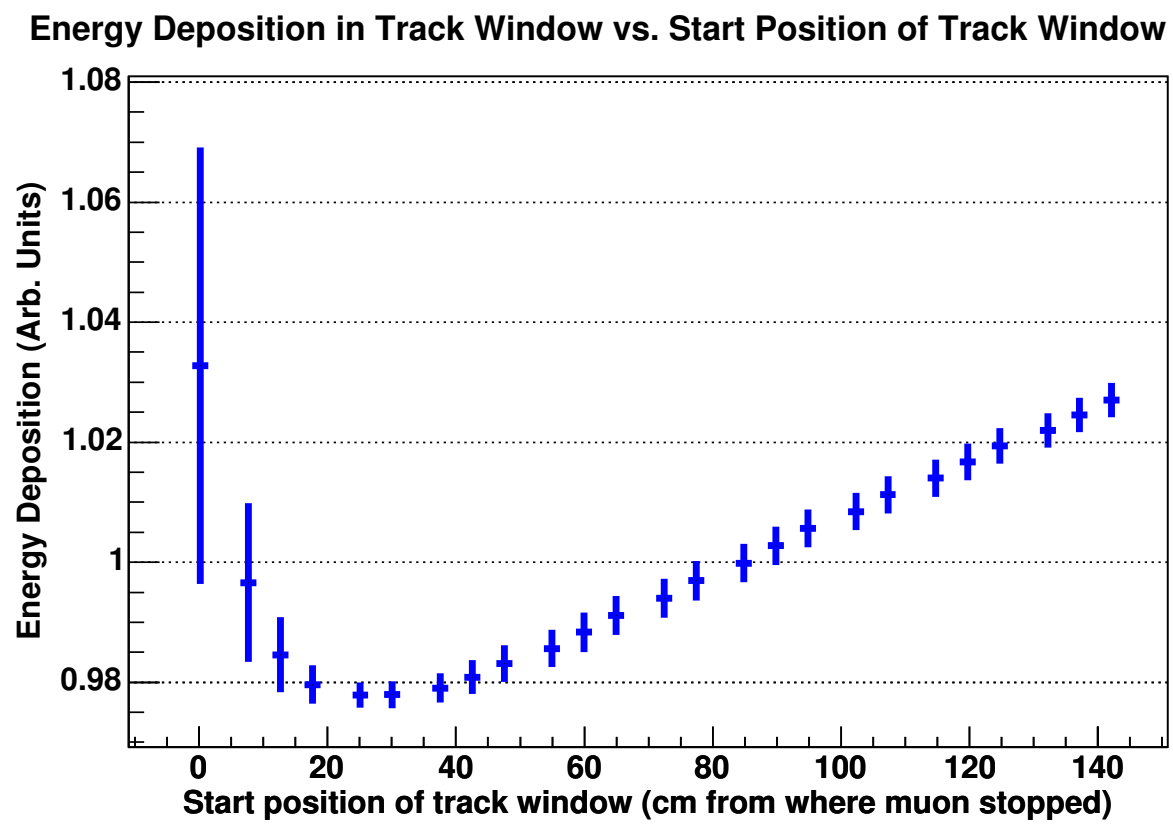

Figure 5.4: Energy deposition in the track window vs. window start position. Each point is the sum of the energy deposited in a track window $83 \mathrm{~cm}$ wide. The error bars represent the spread in the energy deposits within the window range. This plot shows the effect on the track window energy deposition of moving the start position of the window. What's important for the track window technique is the gradient of this plot since that shows how independent of muon track length reconstruction the technique is.

which is well below the $2 \%$ target.

There are a number of reasons that the distance of the window from the end of the track was chosen to be $95 \mathrm{~cm}$ rather than, say, $59 \mathrm{~cm}(10 \times 5.94)$. All the reasons suggest caution rather than being strictly necessary but since there are few reasons not to place the window further away from the end of the track that is what was done (see below).

Various factors such as curvature in the magnetic field, multiple scattering and steep tracks mean that path length reconstruction is more accurate away from the end of the muon track. Not accurately knowing the path length has two implications for the relative energy calibration. Firstly, and most importantly, is that the path length is required to calculate how much scintillator is traversed in the track window and thus measure the detector response. Secondly, the amount of material traversed needs to be known to determine where to position the window on the muon's track. Positioning the window is of secondary importance since the $\mathrm{dE} / \mathrm{dx}$ of the muon is only changing slowly. 
At the Far detector many of the muons have steep tracks since they are coming from above the detector. This means that the distance to the end of the track requires fewer actual physical planes for the required $95 \mathrm{~cm}$ of material to be obtained. For a muon with $\operatorname{Cos}\left(\theta_{z}\right)=1 / 3$ only 5 physical planes would separate the window from the end of the track. If, instead, the window was positioned $59 \mathrm{~cm}$ of material from the end of the track then for a muon with $\operatorname{Cos}\left(\theta_{z}\right)=1 / 3$ that would correspond to only 3 physical planes. The design of MINOS detectors requires several planes to be hit in each view for accurate 3-dimensional reconstruction. This feature of the detector design makes reconstruction near the muon track ends less accurate than in the middle of a long track. As described above, knowing the muon's path length is important when determining the quantity of scintillator traversed in the window. Thus, the presence of steep tracks also suggests positioning the window further away from the end of the track.

\subsection{Stopping Muon Reconstruction}

In order to accurately determine the muon's passage through each plane of scintillator, track reconstruction is performed. Standard track finding algorithms, optimised for each detector, are used to identify the hit strips associated with the muon. A second stage in the reconstruction involves accurately determining the 3-dimensional position of the muon in each plane. Once the position of the muon is well known, the path length through each plane is calculated.

In the case of the Far detector, both the MINOS Standard Reconstruction (SR) software [78] and the "AtNu" reconstruction software (developed with a focus on the atmospheric neutrino analysis) were used for the track finding. To find muon tracks in the Calibration detector the CalDetTracker software package [97] is used. More detailed information on the track finding software used for the Calibration and Far detectors is given separately below in sections 6.1 and 6.2 .

After a track is found, additional detailed reconstruction is performed using the strips 
associated with the track. Each strip has a well known position either along the u-axis or the v-axis depending on the orientation (or view) of its plane, as described in section 3.2.4. In a u-view plane the u-coordinate of a muon's track is determined by knowing which strip was hit. Similarly for a v-view plane. The coordinate that can be measured directly from knowing the strip hit is generically termed the "transverse position" (TPos) for both plane views. The coordinate that can't be measured directly is termed the "longitudinal position" (LPos). The LPos is a measure of the muon's track position along the length of a strip. Calculation of the muon's LPos in a given plane requires extrapolation of the TPos values measured by neighbouring planes of the opposite view.

In extrapolating between positions measured in different planes a linear approximation is made. Thus, curvature in the magnetic field of the Far detector and multiple scattering is neglected. As listed in Table 5.5, a $1 \mathrm{GeV}$ muon travelling through a $1 \mathrm{~T}$ magnetic field has a radius of curvature of $3.5 \mathrm{~m}$. The deflection of such a muon after travelling perpendicularly through 5 planes $(\sim 0.3 \mathrm{~m})$ is $<0.01 \mathrm{~m}$. Such a deflection corresponds to an angle of only $1.9^{\circ}$ and thus has a negligible effect (of order $0.1 \%$ ) on the muon's path length. As the muon slows down the radius of curvature decreases approximately linearly with energy until the muon becomes non-relativistic. What matters for the linear approximation is how much the radius of curvature changes over the region of interest. Even for a $350 \mathrm{MeV}$ muon travelling 5 planes the error on a linear approximation over that range is still $<1 \%$.

Multiple scattering has a negligible effect on the reconstruction of a muon's path length through the detector. From Table 5.4 it can be seen that the multiple scattering of a $1 \mathrm{GeV}$ muon in one plane of steel has an angular width of only $0.9^{\circ}$. Even at $0.2 \mathrm{GeV}$ the multiple scattering only serves to increase the error on the path length of a muon through a plane by approximately $0.3 \%\left(1 / \cos \left(4.6^{\circ}\right)=1.003\right)$.

\section{Track Reconstruction in 3-dimensions}

The three main steps in determining the 3-dimensional position and path length of the muon through each plane are as follows. 
Firstly, any gaps in the track are filled (gaps can occur due to fluctuations in the number of photo-electrons generated at the PMT face). Even if no strip is found by the track finding software in a particular plane, it is necessary to determine the muon's path through that plane. In filling any gaps, the two track views are considered separately and only the TPos is calculated (the position along each strip, given by the LPos, is not calculated at this stage). Small gaps of 1 plane are filled by simply taking the average TPos of strips in neighbouring planes of the same view. Larger gaps of 2 or more planes are filled by linearly interpolating between the nearest planes of the same view that were hit. When the last plane in one of the two track views does not contain a tracked strip a separate procedure is required. In this case, the previous two planes to be hit in the same view are used to linearly extrapolate forwards or backwards.

Secondly, the position of the muon along the length of each strip in the track is determined (the LPos). This is done by simply averaging the TPos of the tracked strips in the planes on either side, which are of the opposite view. The LPos of the muon hits in the planes at the very ends of the track are determined by linearly interpolating forwards or backwards. This interpolation uses the TPos of the tracked strip in the previous plane of the track (end plane-1) and the plane that is three planes from the track end (end plane-3).

Thirdly, once the 3-dimensional position of the muon track in each plane is determined then the path length, $L$, through the plane is calculated. The path length is defined relative to the path length of a perpendicular muon through a plane. As such, the path length is always greater than or equal to 1 , where a path length of 1 corresponds to a muon passing through a plane exactly perpendicularly. Using this definition the path length is simply

$$
L=\frac{1}{\operatorname{Cos}\left(\theta_{z}\right)}
$$

where $\operatorname{Cos}\left(\theta_{z}\right)$ is the angle subtended to the z-axis. To calculate $\operatorname{Cos}\left(\theta_{z}\right)$ the gradient in $\mathrm{UZ}$ and VZ is required as detailed in section 3.2.4. To obtain these gradients a straight line fit to the position of the muon track in groups of 3-5 planes is performed. This fit is done separately for the $\mathrm{u}-\mathrm{z}$ and $\mathrm{v}-\mathrm{z}$ coordinates and hence the two gradients are determined. For 
each plane a new group of 3-5 planes is defined. When the plane is away from the ends of the track, the two planes on each side are used to form a group of 5 . Whereas, at the end of the track there are only neighbouring planes on one side, hence a group of 3 planes is used. It should be noted that in the case of one of the planes in the group not having a hit (i.e. a gap in the track) then position information from the interpolation is re-used in the fit. This causes some of the points in the fit to effectively have additional statistical weight. For a completely straight track this is of no consequence. The alternative to using a position obtained from interpolation is to use a genuine hit that is further away from the plane where the path length is required. There is a trade off in these two techniques. Using hits in planes that are further away means the assumption that the track is straight starts to break down and gives you a bias. Conversely, re-using the information from the interpolation gives a fit that more strongly assumes the track is straight. Given the small impact of curvature in the magnetic field and multiple scattering over a 5 plane region (see the start of section 5.3), the assumption that the track is straight in the track window leads to an error on the path length that is significantly $<1 \%$. In addition, given that the calibration is relative, any bias due to the additional statistical weight of points in the fit would certainly be a second order effect since it would depend on the difference in the number of gaps per track between the three detectors.

Once the 3-dimensional position of the muon in each plane is known the fibre attenuation calibration is applied. The LPos of the muon track in each plane is used to determine the attenuation correction factor for each strip. The same LPos is used to calibrate all the strips that are hit on a given plane, including the cross-talk hits. It is necessary to apply an attenuation correction to the cross-talk hits since the amount of cross-talk increases linearly with the quantity of light hitting the PMT. For example, if the light from a genuine muon hit occurs close to the end of a strip then, say, $50 \%$ more photons will strike the PMT, which causes approximately 50\% more cross-talk. Applying the attenuation correction to all the hits solves this problem. 


\subsection{The Muon Energy Unit}

The results of the inter-detector calibration are expressed by defining a Muon Energy Unit ${ }^{3}$ (MEU). To within the errors of the calibration and the scintillator thickness, $1 \mathrm{MEU}$ of measured detector response corresponds to the same quantity of energy, deposited in the scintillator, at all three detectors ${ }^{4}$. The exact definition of a MEU is given in equation 5.8 but as a rule of thumb, $1 \mathrm{MEU}$ equals the detector response to a perpendicular 1 GeV muon traversing 1 plane of scintillator.

The procedure for measuring the detector response in the track window is as follows. Starting at the end of the stopping muon track the algorithm works backwards from plane to plane summing up the material traversed by the muon. The position of the start of the window is determined when the required $95 \mathrm{~cm}$ (16 planes if perpendicular) of material has been reached. If the required amount of material is reached part way through a plane then that plane is included in the window. The position of the other side of the window (nearest the track vertex) is determined in a similar way. The algorithm continues to work backwards plane by plane until the total sum of the material traversed by the muon reaches the required $95+83=178 \mathrm{~cm}(16+14=30$ planes if perpendicular $)$. Should the required amount of material be reached part way through a plane then that whole plane is included in the window. Once the start and end positions of the track window have been determined the following sum is calculated:

$$
\frac{1}{N_{p}} \sum_{i=1}^{N_{p}} \frac{S_{i}}{L_{i}},
$$

where $S_{i}$ is the total detector response (i.e. the signal in intra-detector calibrated units) in plane $i$ and $L_{i}$ is the path length through that plane (as defined in equation 5.6). The sum is over the $N_{p}$ planes in the track window where $N_{p}<=14$ depending on the steepness of

\footnotetext{
${ }^{3}$ The acronym MIP (Minimum Ionising Particle) is often used as a synonym for MEU, especially since the MINOS off-line software uses MIP ubiquitously. It was felt that the acronym MIP was misleading since the definition of an MEU does not necessarily equal the detector response to an exactly minimum ionising particle. At the Calibration detector the acronym MIP has also been used to refer to the relative calibration procedure carried out using cosmic muons, which was originally used to normalise the different run periods to each other.

${ }^{4}$ Any differences in the thickness of the steel, either within a detector or between detectors, are not accounted for with this calibration. It is only the differences in the response of the scintillator that are corrected for.
} 
the track.

Equation 5.7 gives the detector response in the track window to a single muon. To eliminate the statistical fluctuations and hence obtain an accurate measurement, the centroid of a sample of $\mathrm{N}$ muons is determined. The mean and the median detector response are possible centroids. As will be discussed in section 6.2.4, using the median is advantageous due to the stochastic energy losses that muons can undergo, and also because using the median eliminates the effect of rare but high energy backgrounds. Thus a MEU is defined, incorporating equation 5.7 , as

$$
\operatorname{MEU}=\operatorname{Median}\left(\left(\frac{1}{\mathrm{~N}_{\mathrm{p}}} \sum_{\mathrm{i}=1}^{\mathrm{N}_{\mathrm{p}}} \frac{\mathrm{S}_{\mathrm{i}}}{\mathrm{L}_{\mathrm{i}}}\right)_{1}, \ldots,\left(\frac{1}{\mathrm{~N}_{\mathrm{p}}} \sum_{\mathrm{i}=1}^{\mathrm{N}_{\mathrm{p}}} \frac{\mathrm{S}_{\mathrm{i}}}{\mathrm{L}_{\mathrm{i}}}\right)_{\mathrm{N}}\right)
$$

where $N$ is the number of stopping muons used in the calibration. It's worth noting that an MEU can be defined in many ways but that the systematic errors associated with different methods are likely to be the deciding factor.

The error on the median of a distribution is not well defined, thus in order to assess the error on the value of equation 5.8 a study has been performed. In addition, it is interesting to understand how the error on the median differs from the error on the mean, so this has also been studied. A very large sample of stopping muons $(\sim 80000)$ has been collected at the Far detector (the acquisition of this sample is discussed in detail in chapter 6 but the sample is used here in determining the error on the median). The distribution of detector response in the track window of this sample of muons is shown in Figure 5.5. It can be seen that the distribution is slightly asymmetric with more events on the right hand side of the peak than on the left. Furthermore, on close inspection it can be seen that there are events stretching out all the way along the x-axis on the right hand side of the peak; these events are due to stochastic energy losses of the muon.

In order to determine the error on equation 5.8 the distribution shown in Figure 5.5 is used as a probability density function (pdf). Using this pdf, $\mathrm{N}$ distributions are generated randomly with $\mathrm{x}$ entries each. The median and mean of each of the $\mathrm{N}$ distributions is 


\section{Detector Response in Track Window}

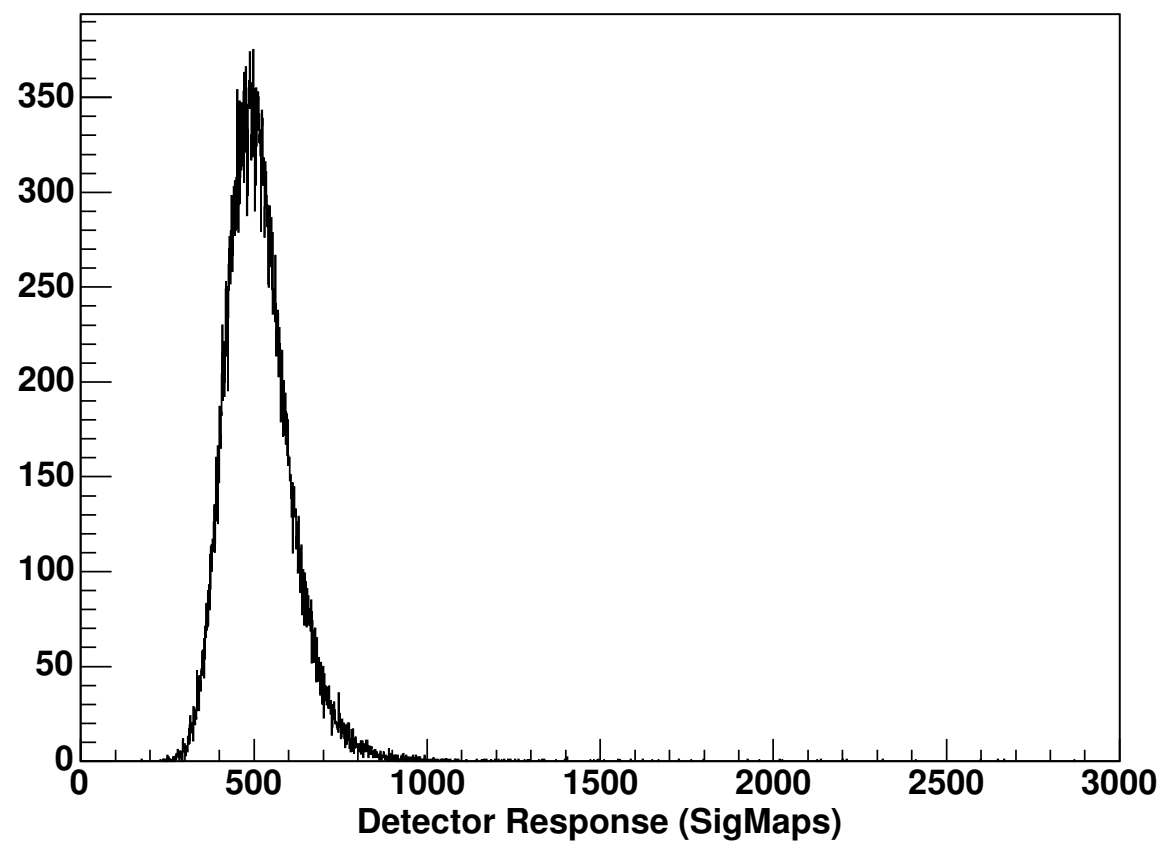

Figure 5.5: Detector response in track window. This plot shows the distribution of the detector response in the track window for $\sim 80000$ stopping muons in the Far detector. It can be seen that the distribution is slightly asymmetric with more events on the right hand side of the peak than on the left. Furthermore, on close inspection it can be seen that there are events stretching out all the way along the $\mathrm{x}$-axis on the right hand side of the peak. 


$\begin{array}{cccc}\begin{array}{c}\text { Number of } \\ \text { entries, }\end{array} & \begin{array}{c}\text { Fractional error } \\ \text { on median }\end{array} & \begin{array}{c}\text { Fractional error } \\ \text { on mean }\end{array} & \begin{array}{c}\text { Fractional error } \\ \text { on mean (Gaus.) }\end{array} \\ 50 & 0.030 & 0.028 & 0.027 \\ 100 & 0.021 & 0.020 & 0.019 \\ 200 & 0.015 & 0.014 & 0.013 \\ 500 & 0.010 & 0.009 & 0.009 \\ 1000 & 0.007 & 0.006 & 0.006 \\ 5000 & 0.003 & 0.003 & 0.003\end{array}$

Table 5.6: Error on the MEU value using the median or mean. This table gives the results of a study into the error on the median and the mean of the distribution shown in Figure 5.5 when distributions with $\mathrm{x}$ entries are used. In addition, the errors calculated with the assumption that the distribution in Figure 5.5 is Gaussian are given.

determined, then the fractional deviation of the mean and the median w.r.t. to the true values obtained from the pdf is calculated. A histogram of the $\mathrm{N}$ fractional deviations is made and the width of the resulting distribution is used to determine the fractional error on the median and the mean. A large value of $\mathrm{N}$ is used (25000) in order to reduce the error on the fractional error to a negligible value. Table 5.6 gives the error on the median and the mean for distributions of a varying number of entries as well as the calculated error on the mean assuming the distribution in Figure 5.5 is Gaussian. There are a number of interesting points to make about the data in the table. Firstly, that the error on the median is $10-13 \%$ larger than the error on the mean for the number of entries shown. Secondly, the error on mean of the distribution in Figure 5.5 is the same as the error that would be obtained assuming a Gaussian distribution to $\sim 3 \%$.

The data in Table 5.6 shows that the loss of precision in using the median instead of the mean is only $\sim 10 \%$ when using the same sized sample. However, since the number of stopping muons obtained in each of the detectors is $>1000$ the increase in the size of the statistical error when using the median is negligible. 


\section{Chapter 6}

\section{Relative Energy Calibration Results}

In this chapter the results of the relative energy calibration in the Calibration detector and the Far detector are presented (see sections 6.1 and 6.2 respectively). For each detector, descriptions of the stopping muon selection and the backgrounds to the sample are given. The results of the calibration are then presented before the systematic errors are described in detail.

Section 6.3 describes how measurements of the shape of the Bethe-Bloch curve are used to cross-check the stopping muon reconstruction and calibration in the two different detectors. Section 6.4 details how the relative calibration between the Far detector and Calibration detector was performed in MC as a valuable cross-check of the relative calibration procedure.

\subsection{The Calibration Detector}

The stopping muons used at the Calibration detector are 1.4-2.0 GeV muons from the PS accelerator T7 and T11 beamlines. The Calibration detector's small size means that its acceptance to cosmic stopping muons is low. Furthermore, backgrounds from through-going cosmic muons, that appear partially contained, are high. Thus, it is only possible to use beam stopping muons for the Calibration detector [77]. 
Results of a relative calibration, that has been carried out for three Calibration detector run periods (T11 2002, T7 2002 and T7 2003), are presented in this chapter.

\subsubsection{Stopping Muon Selection and Backgrounds}

Selection of beam muons between $1.4-2.0 \mathrm{GeV}$ is relatively straightforward at the Calibration detector. There are essentially two main steps in the selection procedure: firstly, the PID information from Cerenkov counters and TOF system are used; and secondly, event length requirements are made. More detailed timing and tracking information is used to reject backgrounds and ensure that the muons are contained in a fiducial volume.

In the $\mathrm{T} 7$ and $\mathrm{T} 11$ test beams there are four abundant particle types: electrons, muons, protons and pions. Electrons are identified with high efficiency (typically $>99.9 \%$ ) using the Čerenkov counters [68]. At the beam momenta required for stopping muons, the protons are clearly identified ( 100\%) using the time-of-flight (TOF) system [68]. Figure 6.1 shows the TOF spectrum for a $1.4 \mathrm{GeV} / \mathrm{c}$ run where the proton peak is clearly separated from the other particle types. The TOF system is also used to remove spurious events by checking that the speed of the electrons, pions and muons is nearly that of the speed of light. The timing resolution of the TOF system employed is not precise enough to actually discriminate between electrons, pions and muons at the momenta considered. Muons can be tagged with the Čerenkov counters when they are set at a high pressure but the efficiency is low. Relatively few runs were taken with these high pressure Čerenkov settings so this information has not been used to select muons in this thesis.

The CalDetPID software package [70] is used by this author as the first step in identifying the particle type responsible for each event. The package uses database tables that contain information on the Čerenkov and TOF signals consistent with each particle type. For each event a bit-packed array of the possible particle types is produced, which is then used in the selection.

As discussed above, the TOF and Čerenkov systems can identify electrons and protons 


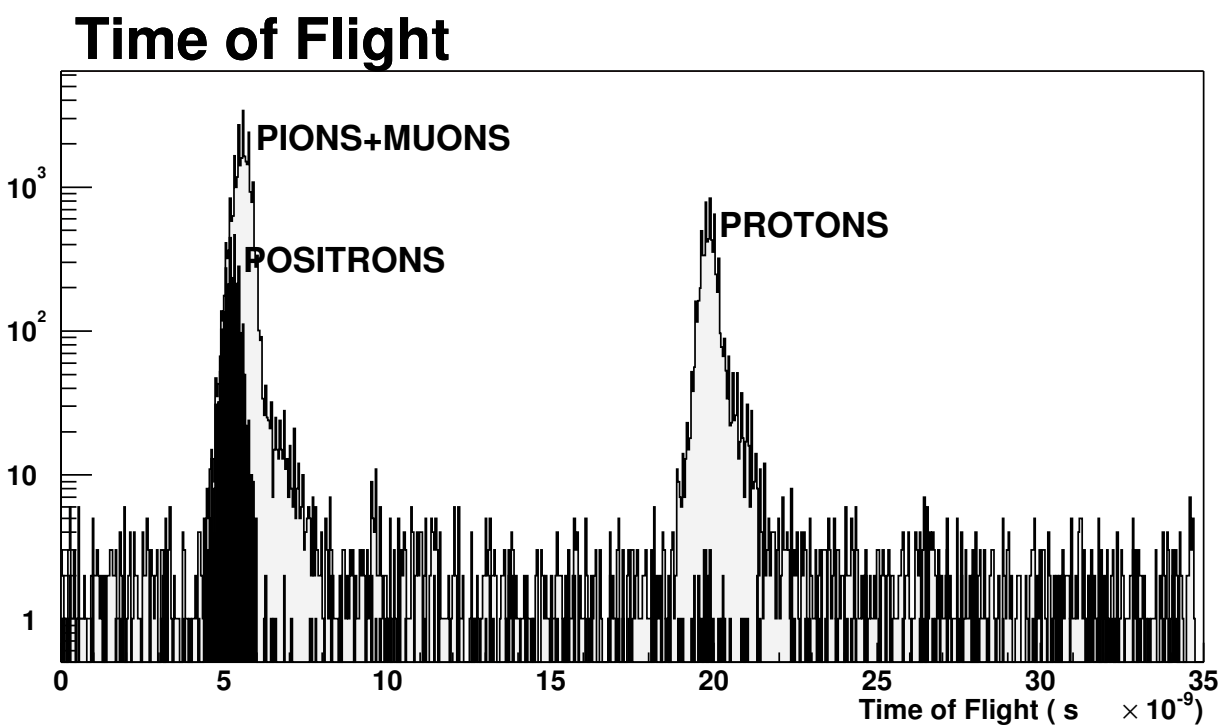

Figure 6.1: Time-of-flight spectrum from the Calibration detector TOF system. This is the TOF spectrum from a $1.4 \mathrm{GeV} / \mathrm{c}$ run in the T11 test beam. The proton peak is clearly separated from the highly relativistic positrons, pions and muons. The positrons were independently identified using the Čerenkov counter and are seen to be slightly quicker than the pions and muons. Figure from [69].

but cannot always distinguish muons and pions. However, the muons can be separated from the pions with high efficiency using an event length cut. Table 6.1 gives the event length cuts for different beam energies. The event length cuts are chosen by calculating a certain fraction of the distance travelled by the average muon: the lower cut is placed at approximately $80 \%$ of the distance travelled at each particular energy; and the upper cut is placed at approximately $115 \%$ of the distance travelled (except at $2.0 \mathrm{GeV}$ where a small fraction of the muons exit the detector. See section 6.1.3). Figure 6.2 shows the number of planes hit for muons and pions in a MC simulation at $1.8 \mathrm{GeV}$. This figure illustrates that the vast majority of the pions are well separated from the muons at this energy. Figure 6.3 shows the number of planes hit in data at 4 different beam energies, only events that have passed the PID cuts i.e. muons and pions are shown. It is interesting to observe the trend with increasing energy: the number of planes hit by the pions with changing beam energy is relatively static compared to the muons. The most probable number of planes hit by the muons changes from approximately 40 planes at $1.4 \mathrm{GeV}$ to 56 planes at $2.0 \mathrm{GeV}$. It is also worth noting that a sharper peak in the number of planes hit by the muons forms in the MC. In the data the number of planes hit by the muons rises early and steadily. The production 


$\begin{array}{ccc}\mathbf{P}(G e V / c) & \begin{array}{r}\text { Lower Event Length } \\ \text { Cut (Plane) }\end{array} & \begin{array}{c}\text { Upper Event Length } \\ \text { Cut (Plane) }\end{array} \\ 1.4 & 31 & 45 \\ 1.6 & 35 & 51 \\ 1.8 & 39 & 56 \\ 2.0 & 44 & 57\end{array}$

Table 6.1: Event length cuts for muons of different energies at the Calibration detector. The cuts are inclusive and thus muons stopping exactly in the planes listed are included in the sample. Note that the planes are numbered from zero, so the actual event length is always 1 greater than the last plane hit.

\section{Number of Planes Hit by Muons and Pions}

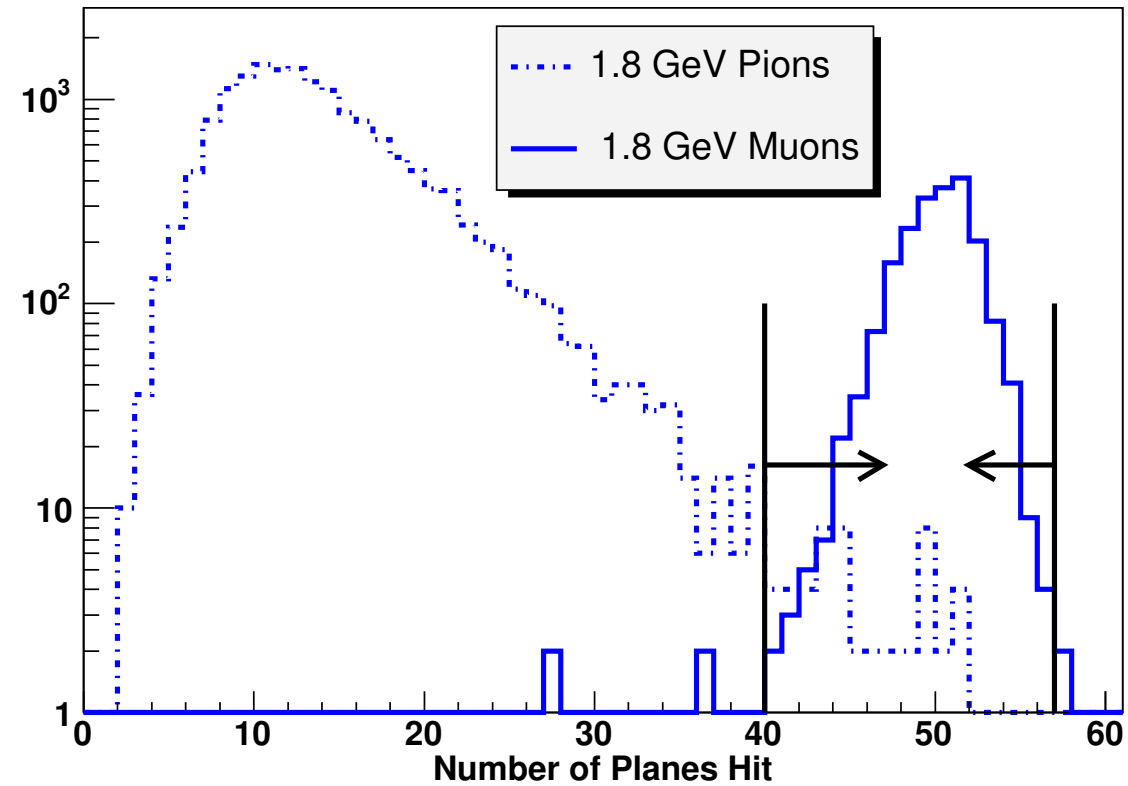

Figure 6.2: Number of planes hit by muons and pions in a GEANT3 MC simulation at $1.8 \mathrm{GeV}$. The ratio of pions to muons shown here is $8: 1$, which is approximately the testbeam composition. The position of the event length cuts is shown by the black line.

of these lower "off-momentum" muons is understood to arise from the decay kinematics of the pions in the beamline [68].

\section{Backgrounds to Stopping Muon Sample}

The major background to the stopping muon sample comes from overlapping events: muons not coming directly down the beam-pipe and multiple particles from the beamline can enter the Calibration detector very close in time. Pions also constitute a background but the vast majority are removed by the event length cuts. The systematic effect of the different 
Number of Planes Hit at $1.4 \mathrm{GeV}$

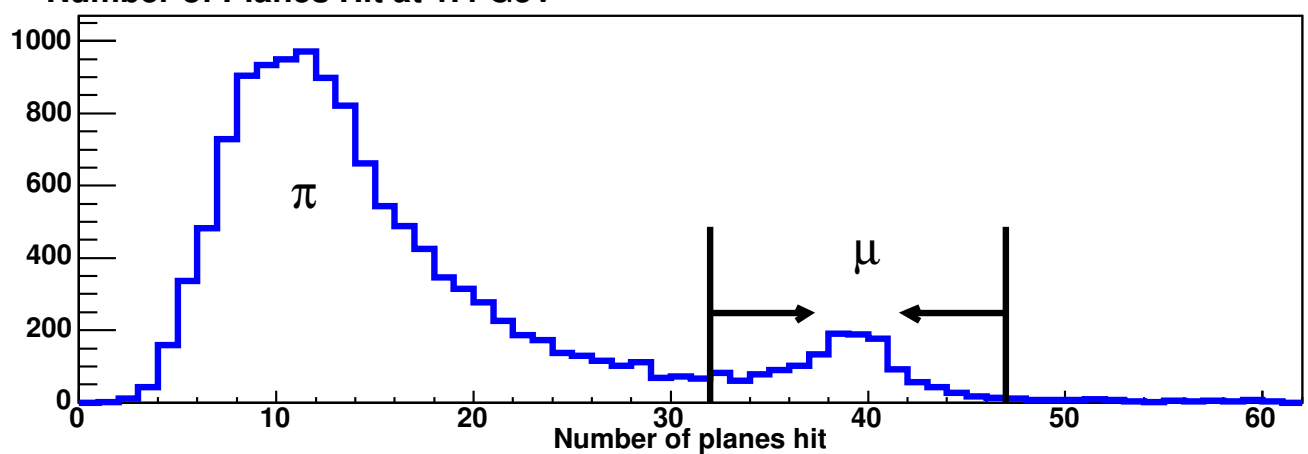

Number of Planes Hit at $1.6 \mathrm{GeV}$

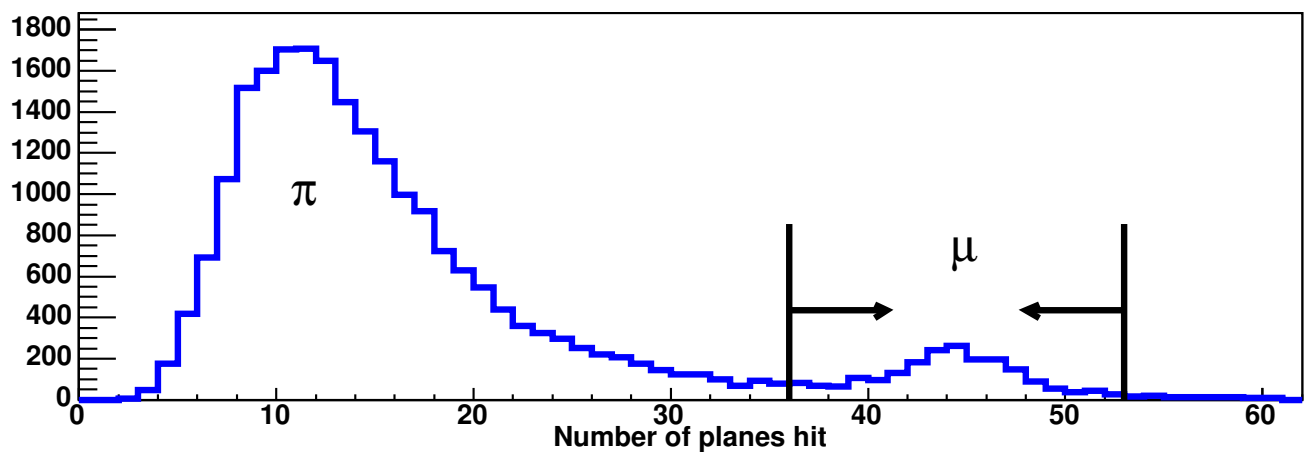

Number of Planes Hit at $1.8 \mathrm{GeV}$

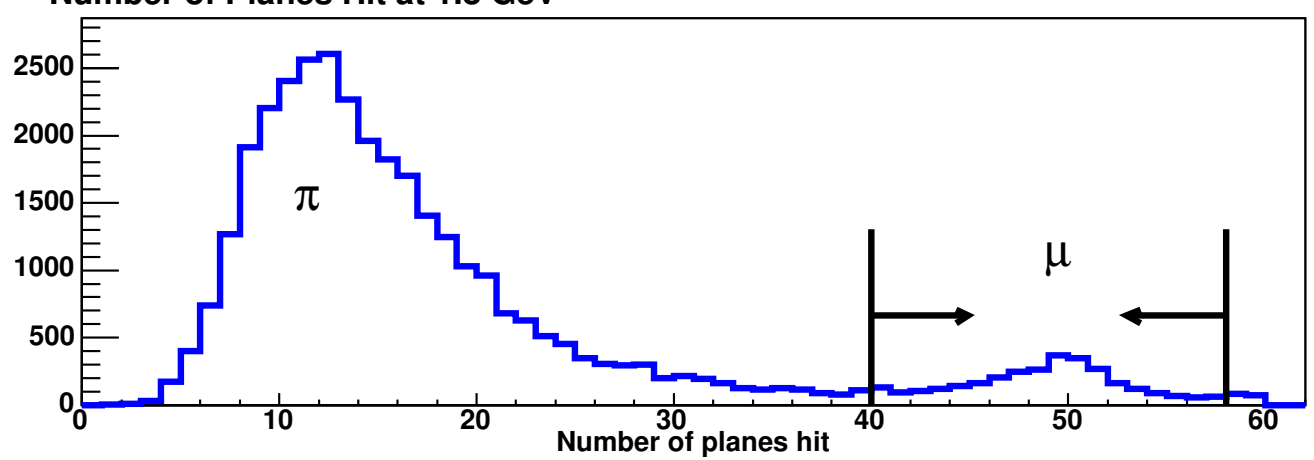

Number of Planes Hit at $2.0 \mathrm{GeV}$

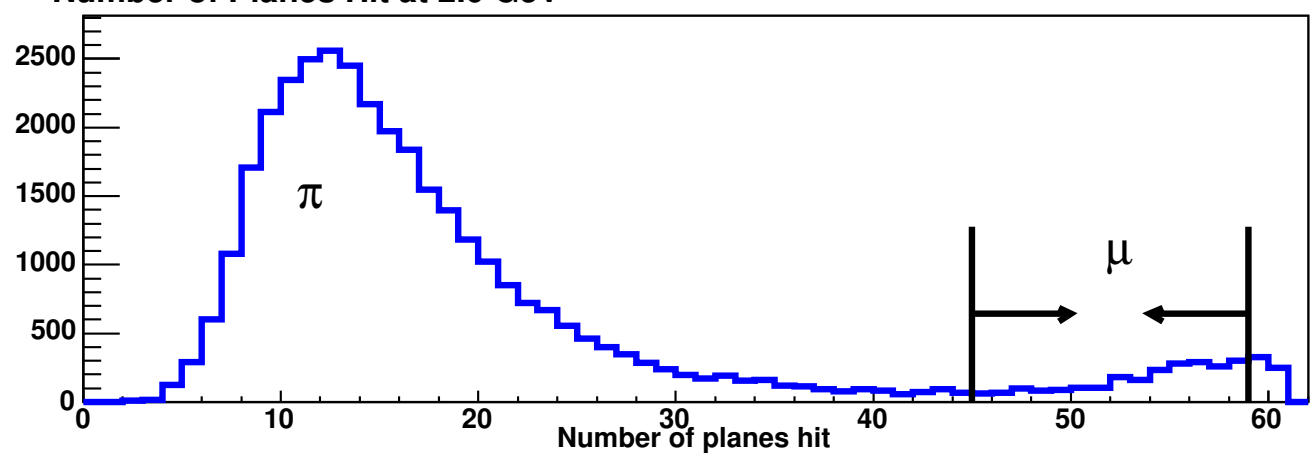

Figure 6.3: Number of planes hit by muons and pions between 1.4-2.0 GeV. The only cut applied to the data was the PID in order to remove electrons and protons. The black lines show the position of the event length cuts used to select muons. In the bottom plot, showing $2.0 \mathrm{GeV} / \mathrm{c}$ data, there is an anomalous peak at around 59/60 planes hit. This is due to a "pile-up" effect caused by muons exiting the end of the detector [98]. 
backgrounds is considered in detail in section 6.1.3.

"PS muons" [99] come not directly down the beam-pipe but instead by punching through the concrete shielding around the test-beams. In the $\mathrm{T} 7$ test-beam these muons are parallel to the particles from the beam-pipe and travel in the horizontal plane. However, in the T11 test-beam these muons come at an angle to the detector relative to the beamline while still travelling in the horizontal plane. The PS muons strike the whole face of the detector and in T11 they strike the side of the detector as well. Using the angular information from the two test-beams a triangulation-type technique suggests that source of the muons is close to where the primary beam is extracted from the PS accelerator.

The Calibration detector is externally triggered by the TOF system, hence only particles coming directly down the beam line can initiate data taking. The PS muons enter the stopping muon sample by hitting the detector at the same time as a "real" beam event, say an electron or a pion. Typically, the track finding algorithm will locate the long straight PS muon track rather than the genuine, often shower-like beam event.

Due to the need to record many thousands of events of each particle type at many energies the beamline was run at a moderately high rate. One consequence of this is that the probability of two events coming down the beamline at the same time and "overlapping" in the detector is increased. These overlapping events often have double the expected detector response and thus need to be removed.

\section{Background Rejection Cuts}

The techniques used to reject the backgrounds from overlapping events involve the timing of PMT signals and pattern recognition in the form of track-finding.

Track reconstruction at the Calibration detector is performed with the CalDetTracker software package [97]. The tracker has two distinct algorithms: the first is used for tracking straight cosmic muons [77]; and the second is used for tracking beam particles [38] that may come to a stop in the detector and curve substantially as they do so. For this thesis the 
beam particle tracking algorithm was used.

The timing of the PMT signals provides a powerful way of identifying many overlapping events. However, not all overlapping events can be separated using timing for two main reasons: firstly, the overlapping events may occur very close in time; and secondly, the Far detector electronics only records the time of the first dynode signal, later hits on the same PMT have the same time recorded as the first hit.

The following cuts are applied to the stopping muon event sample:

- Fiducial volume cuts

- The track must not extend into the last two planes of the detector (numbered $58 \& 59)$

- The track must not extend into the outer 3 strips of the detector on all 4 sides (only strips with signal greater than 1.5 photo-electrons are considered in the cut).

- The average tracked strip in the first 4 planes (numbered 0-3) of the detector must be between strips 9-12 inclusive such that the track is consistent with a beam particle entering the detector.

- Timing cuts

- The time of every scintillator hit in an event must lie between -35 and $295 \mathrm{~ns}$ of the TOF system trigger time (TOF time offsets are listed in Table A.1).

- Track reconstruction cuts

- A track must be reconstructed in both views.

- Track quality cuts

- The number of hits per plane with a signal greater than 1.5 photo-electrons in the first 10 planes must be between 1.1 and 2.7. 


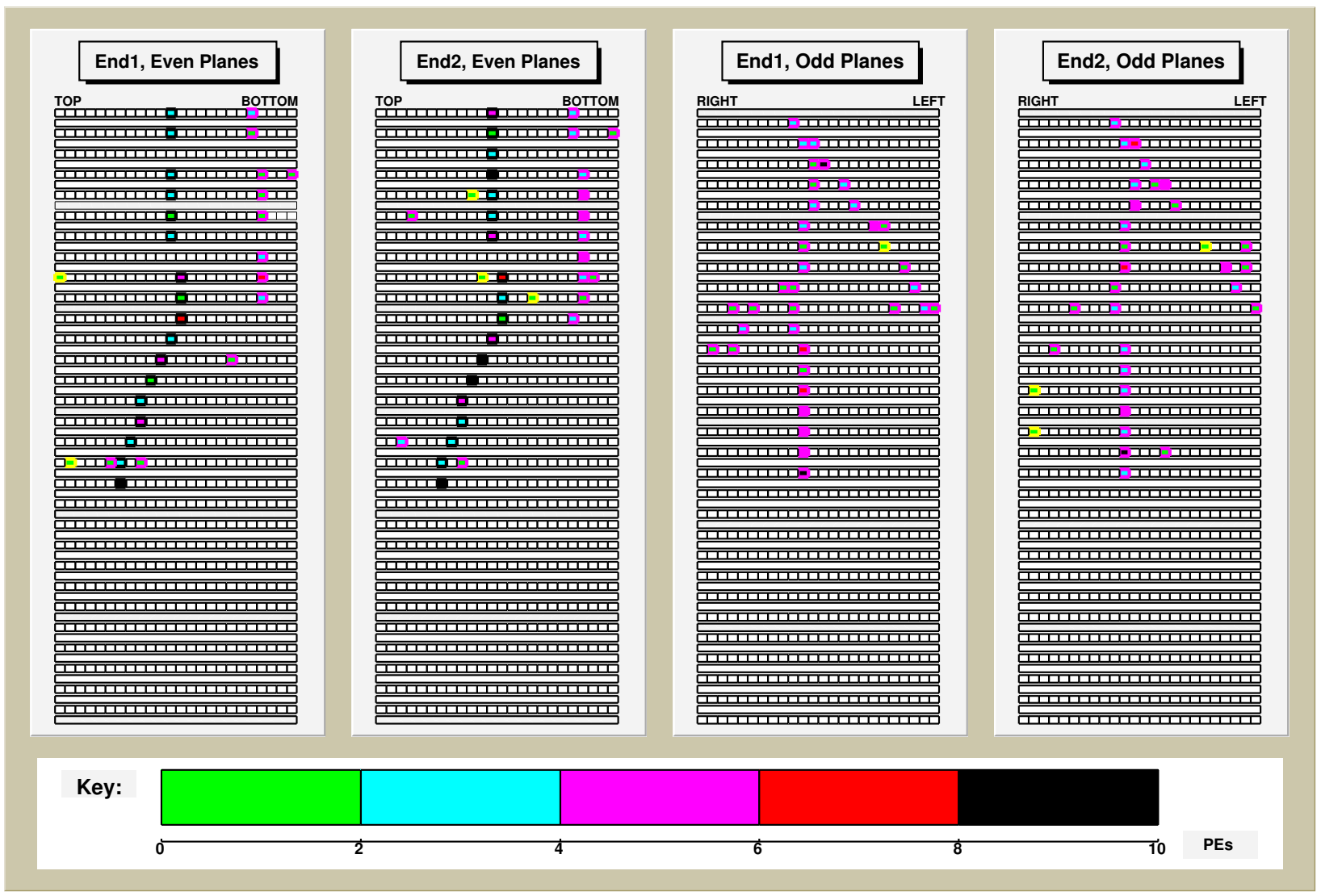

Figure 6.4: An overlapping "PS muon" and test-beam muon. This event display has four panels that display the information from the two views (even or odd planes) and the two ends of each strip. Tracked hits are shown with a black outline, cross-talk hits with a yellow outline and untracked hits with a pink outline; the fill colour of the hits shows their charge in photo-electrons. The event shown is 543 in run 40817; it contains a beam muon entering the detector at the top of the page and coming to a stop nearly $2 / 3$ s of the way down the detector; overlapping with the beam muon is a PS muon that enters the detector to the bottom right of the beam spot before travelling diagonally across the detector and exiting $1 / 3$ of the way down. The lack of hits with a black outline in the odd planes shows that the tracking algorithm was confused by the presence of two tracks and failed to reconstruct the event in that view.

- The number of hits per plane in the whole event must be between 1.7 and 2.9.

A large number of the PS muons are removed by the fiducial volume cuts since they most often enter the detector away from the beam spot and they always exit the detector. However, the presence of two particles, especially two muons, makes the tracks harder to recognise and hence the tracker will often fail. An example is shown in Figure 6.4.

Overlapping events involving two beam particles are rarely removed by the fiducial volume cuts. The ratio of muon content of the beam to pions + protons + electrons is of the 
order of 1:20. Any overlapping event passing the event length cut is unlikely to consist of two muons from the beam line but rather one muon with one showering particle. The timing cut can remove such events as long as they are separated by a few tens of nanoseconds and not all the same PMTs are struck. However, in the case that the timing cut fails, the cut on the number of hits per plane in the first 10 planes with signals greater than 1.5 photo-electrons is effective. This cut on the number of hits per plane is specifically designed to remove events involving a muon and a showering particle that doesn't penetrate very deep into the detector.

\section{Effectiveness of Cuts}

A comparison of the relative effectiveness of each of the different cuts used to select the stopping muon sample and reject background events is made here.

Figure 6.5 shows how the time-of-flight distribution and the ADC spectrum of a Čerenkov counter are used to select muons. It can be seen that the Čerenkov counter only rejected one event in this run, which probably involved an electron overlapping with a muon. The TOF system rejects about $5 \%$ of events and although some of these may be good stopping muons it is a sensible precaution to reject events where there is clearly something erroneous about the measured time-of-flight.

Figure 6.6 shows the effect of each of the topological and temporal cuts used in select the stopping muons. Clearly the most effective cut is the event length cut, shown in the top left plot, which removes the vast majority of the pions. The middle left plot shows the maximum tracked strip position relative to the centre of the detector; it can be seen that approximately $15 \%$ of events have a track that leaves the fiducial volume at some point. The plots for the other four cuts show that these cuts remove relatively few events when they are applied last in the sequence. However, because all four cuts are designed to remove overlapping events and no one measure is $100 \%$ efficient they are all required. A scan by eye of the events removed showed that in nearly every case the rejected event involved overlapping particles. An example of a PS muon overlapping with a beam muon is shown in Figure 6.4. 


\section{Time of Flight}

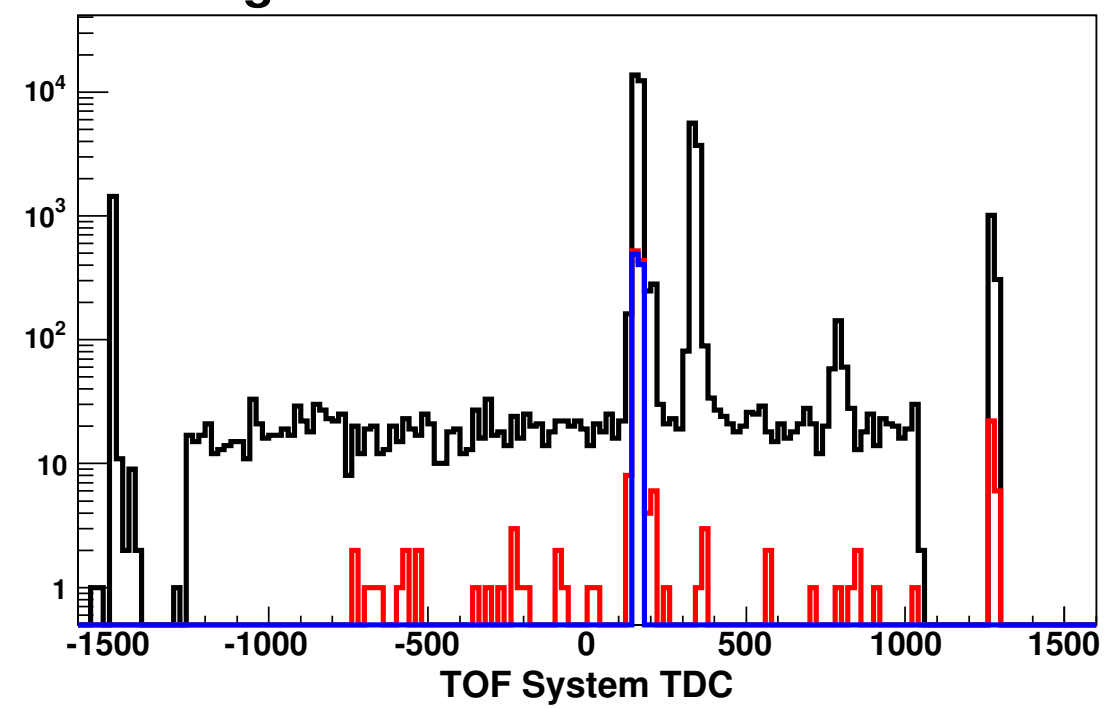

\section{Cerenkov Counter ADC}

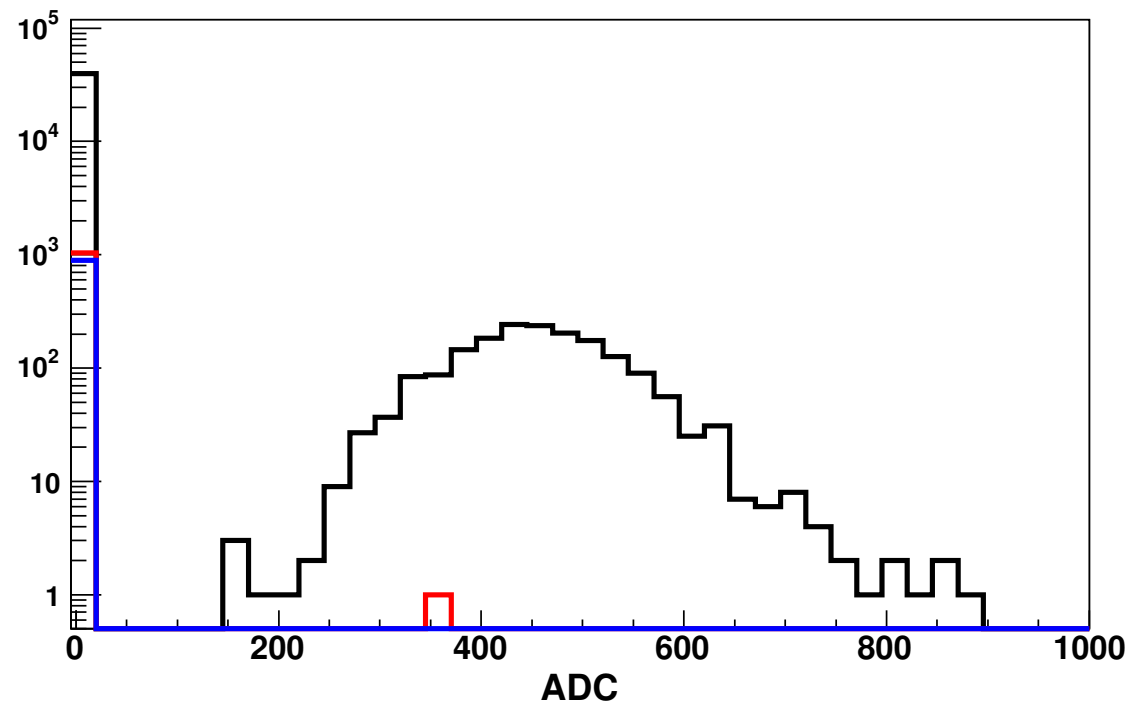

Figure 6.5: Particle identification cuts at the Calibration detector. Histograms of the measured time-of-flight and Čerenkov counter ADC distributions are shown for run 40817, which had a momentum setting of $1.6 \mathrm{GeV} / \mathrm{c}$. The raw distribution resulting from particles of all types is shown in black, the distribution resulting from making all but the PID cuts is shown in red, and lastly the distribution after making all the cuts is shown in blue. 


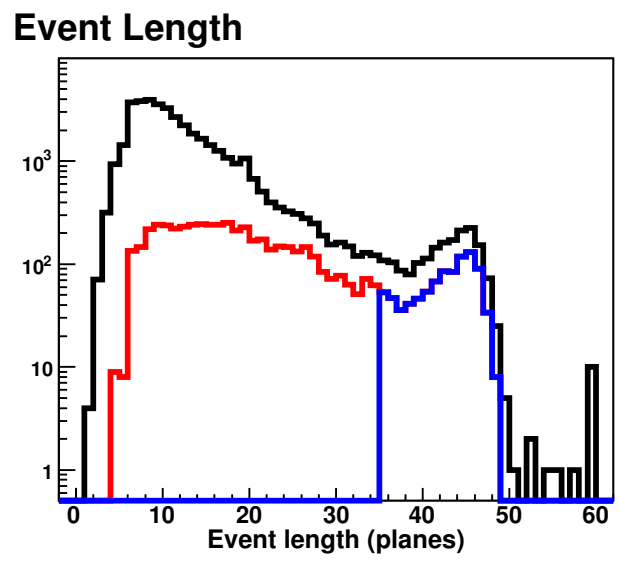

Max. Track Position

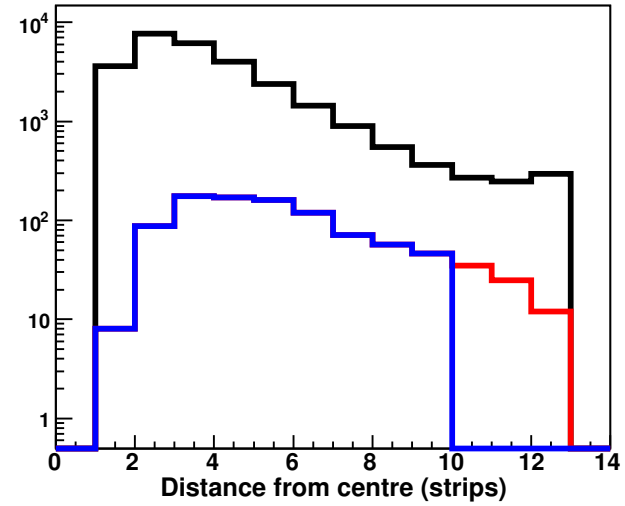

Av. Hits per Plane (First 10)

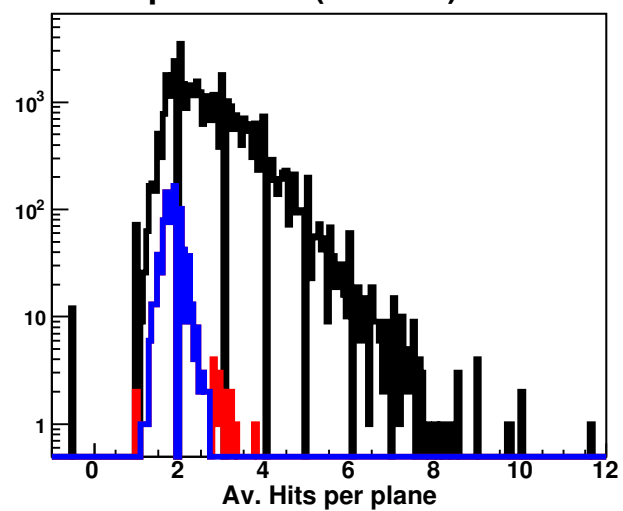

Scint.-Trigger Time Diff.

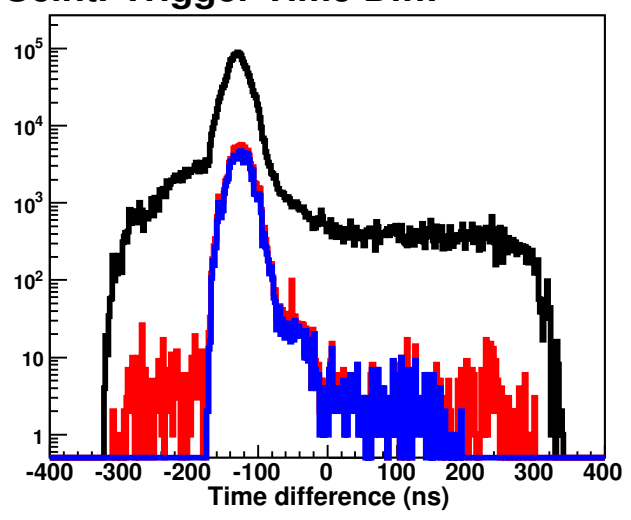

Track Entry Point

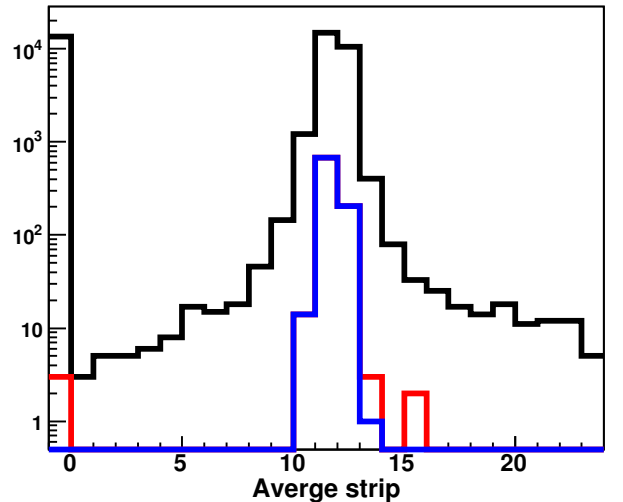

Av. Hits per Plane (Whole)

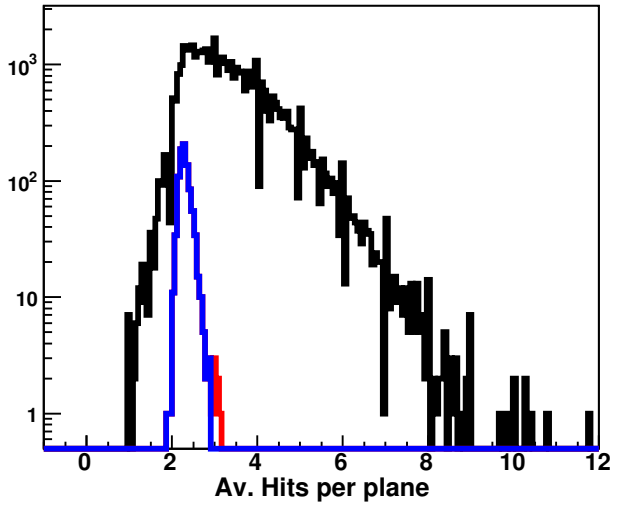

Figure 6.6: Event selection and background rejection cuts at the Calibration detector. Histograms of the cut quantities for run 40817 that had a momentum setting of $1.6 \mathrm{GeV} / \mathrm{c}$ are shown. The top left plot shows the event length distribution; the top right plot shows the difference between the time of individual scintillator hits and the TOF trigger time (note that the times for all the hits are included; an event is cut if any one of the hits is outside the allowed range); the middle left plot shows the maximum distance from the detector centre of a tracked strip (fiducial volume cut); the middle right plot shows the average tracked strip in the first 4 planes; the bottom left plot shows the average hits per plane with a signal greater than 1.5 photo-electrons in the first 10 planes; finally, the bottom right plot shows the average hits per plane in the whole event. For each cut quantity, the raw distribution resulting from particles of all types is shown in black, the distribution resulting from making all but that particular cut is shown in red, and lastly the distribution after making all the cuts is shown in blue. 
The systematic effect of all the cuts used in this analysis to select stopping muons and reject backgrounds is evaluated in section 6.1.3.

\subsubsection{Results}

The results of relative calorimetric energy response measurements for the Calibration detector are presented in this section. The detector response has been determined for three run periods: T11 2002, T7 2002 and T7 2003. Table A.2 lists the runs used in the calibration. Table A.3 lists the runs that are not used and the reasons why.

The results given for the T7 2003 run period are those for the "Near-Far" configuration. In the "Near-Far" configuration the Far detector instrumentation (PMTs and electronics) is used to read out one end of each scintillator strip and Near detector instrumentation to read out the opposite end.

The results stated in this section are given in "SigMap" units, which is the unit of detector response obtained after the full intra-detector calibration is applied. Although, strictly speaking, the results are given after only the strip-to-strip calibration has been applied. The unit of strip-to-strip calibrated ADCs is called "SigCor". The fibre attenuation calibration does not significantly change the measured MEU value (SigMap $\simeq$ SigCor) for the Calibration detector. The small systematic effect of not performing the attenuation correction is discussed in section 6.1.3.

To determine the MEU value, equation 5.8 is used. The MEU value is calculated for each run and the results are shown graphically in Figure 6.7. The red circles, green squares and blue triangles show the MEU values for runs in the T11 2002, T7 2002 and T7 2003 run periods respectively.

Comparing the scatter of the points in Figure 6.7 with the error bars reveals that there is a source of variation above that expected from statistics alone. A fit to a constant for each of the three run periods gives $\chi^{2} / n d f=59 / 6,1.5 / 2,67 / 23$ for the T11 2002, T7 2002 and T7 2003 run periods respectively. The fit for the T7 2002 run period is slightly over constrained 
Stopping Muon Response vs. Run

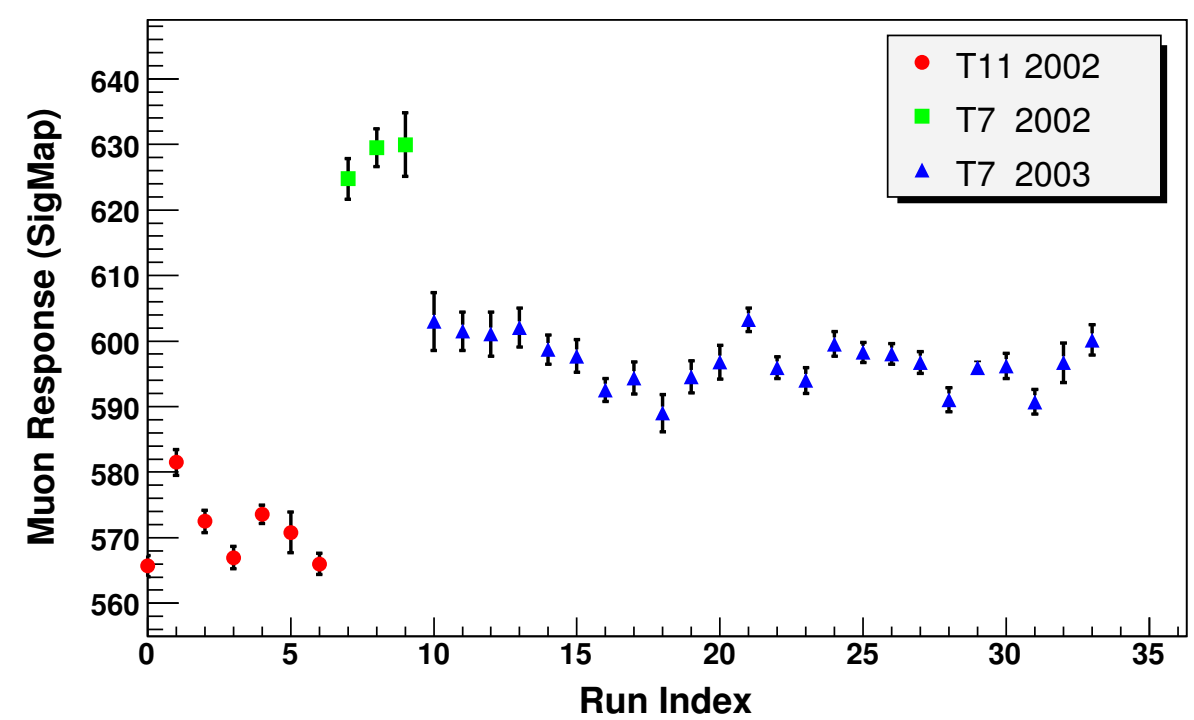

Figure 6.7: Calibration detector response to stopping muons vs. run index. The red circles, green squares and blue triangles show the MEU values for runs in the T11 2002, T7 2002 and T7 2003 run periods respectively. The error bars show only the statistical errors.

but given there are only 3 points the probability of this occurring is $47 \%$. The other two run periods clearly show a source of variability not described by the statistical error bars, although it is worth noting that the fit to the T11 2002 data is made significantly worse by the presence of the second data point. To assess the size of the additional variability an additional error is added in quadrature to the statistical error until the $\chi^{2} / n d f$ is unity. A $0.9 \%$ and $0.5 \%$ error were required to account for the additional variability in T11 2002 and T7 2003 run periods respectively. Although, again, it should be noted that the additional error for the T11 2002 run period is significantly increased by the presence of the second data point. A full analysis of the source of the different systematic errors is presented in section 6.1.3.

The final MEU value is taken as an average of all the runs in each run period. Table 6.2 lists the average MEU value in SigMap units for the three different run periods. Both the statistical and systematic errors are quoted. 


$\begin{array}{cc}\text { Run Period } & \text { MEU (SigMap) } \\ \text { T11 2002 } & 571.0 \pm 0.7 \text { (stat.) } \pm 7.4 \text { (syst.) } \\ \text { T7 2002 } & 628.1 \pm 2.1 \text { (stat.) } \pm 8.2 \text { (syst.) } \\ \text { T7 2003 } & 597.0 \pm 0.5 \text { (stat.) } \pm 7.8 \text { (syst.) }\end{array}$

Table 6.2: MEU values for Calibration detector run periods. Both statistical and systematic errors are quoted. A full analysis of the different systematic errors is presented in section 6.1.3

\subsubsection{Sources of Systematic Error}

There are various different ways in which systematic errors can affect the relative calibration. Broadly speaking the effects can be considered in three ways. Firstly, do they affect the MEU value obtained from run to run within a run period? Secondly, was there a problem affecting a particular run period with respect to the others? Thirdly, is there a systematic effect introducing an artificial offset to the measured energy scale at the Calibration detector with respect to one of the other two detectors? Many potential sources of systematic error are evaluated below.

\section{Fibre Attenuation}

The fibre attenuation correction is not used for the relative energy calibration at the Calibration detector because the beam muons pass within $\pm 20 \mathrm{~cm}$ of the centre of each strip in the track window. The response of a strip, when the sum of both stripends is considered, changes by only $\pm 1.0 \%$ in this region. The muons are on average located within 1 strip $(4.1 \mathrm{~cm})$ of the detector centre. Thus, the effect of not performing the attenuation calibration is a systematic bias on the MEU value of $\sim 0.2 \%(20 \mathrm{~cm} / 4.1 \mathrm{~cm} \times 1 \% \simeq 0.2 \%)$ and a slight decrease in the calorimetric energy resolution. These effects are thus small enough not to have a significant effect on the measurement of the detector response and hence the relative calibration. 


$\begin{array}{ccccc}\begin{array}{c}\text { Run Period } \\ \text { All }\end{array} & \begin{array}{c}\text { Plane } \\ 0\end{array} & \begin{array}{c}\text { Strip } \\ \text { All }\end{array} & \begin{array}{c}\text { Side } \\ \text { Both }\end{array} & \begin{array}{c}\text { Problem } \\ \text { Too noisy }\end{array} \\ \text { T7 \& T11 '02 } & 35 & \text { All } & \text { West } & \text { Low light level } \\ \text { T7 \& T11 '02 } & 21 \& 41 & 19 & \text { East } & \text { Bad pedestal } \\ \text { T7 '03 (Part) } & 21 \& 41 & 19 & \text { East } & \text { Bad pedestal } \\ \text { T7 \& T11 '02 } & 20 \& 40 & 4 & \text { West } & \text { Bad pedestal }\end{array}$

Table 6.3: Detector regions with readout problems at the Calibration detector. The regions of the detector affected by readout problems in the different run periods and the problem that occurred are listed.

\section{Detector Readout Problems}

During the different data taking periods, problems with the readout occurred in different regions of the detector. The effect of these problems on the MEU calibration has to be assessed.

Table 6.3 lists the regions of the detector that had readout problems in the different run periods and what the problem was. The channels that had a bad pedestal value are simply removed from each event; their location in the detector means that they are rarely hit and only contribute a tiny fraction of the total detector response. Figure 6.8 shows the fraction of the total MEU value contributed by each strip in the track window on average. It can be seen that removing a central strip would have a $1.8 \%$ effect on the MEU value, whereas removing a strip such as 19 only contributes $<0.01 \%$, which is completely negligible. However, in the case where the readout of a whole plane is problematic, a correction is needed. Planes in the centre of the track window typically contribute 1/14th of the MEU value. In 2002 data there is a problem with plane 35 not reading out properly and a correction is required. The response in the neighbouring planes is used to determine what the response of plane 35 should be and that value is used. The error associated with this procedure is not significant.

A dead-time less system of recording the particles from the test-beam was developed using the TOF system as a trigger to enable the detector [68]. Running the electronics continuously in their dynode triggering mode is not possible due to the noisy test-beam environment and the high rate of cosmic muons, since each time a PMT dynode triggers a $5 \mu s$ dead-time is occurred due to the digitisation time. With the TOF triggering system in 

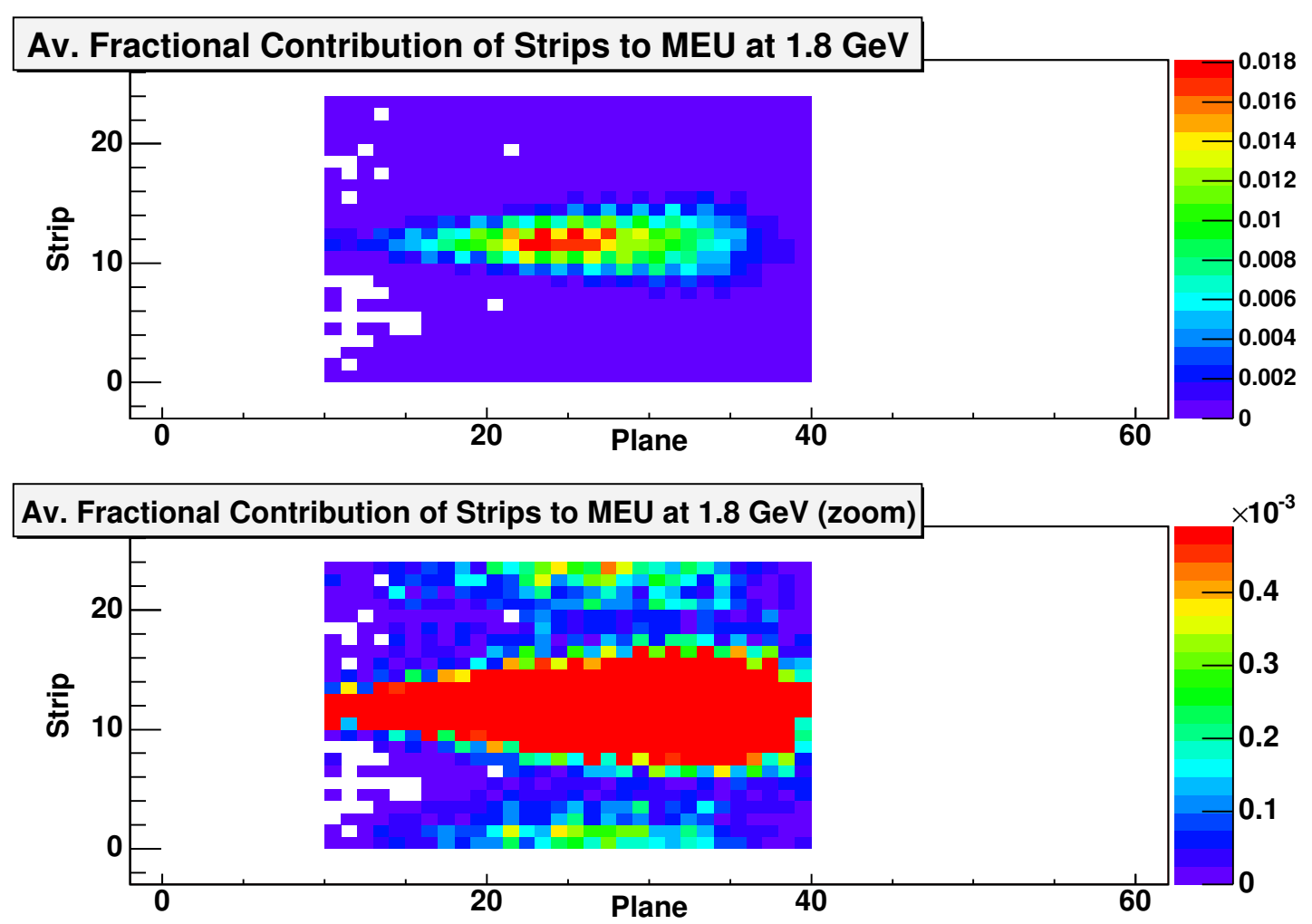

Figure 6.8: Average fractional contribution of individual strips to MEU at $1.8 \mathrm{GeV}$. For every stopping muon the fraction of energy in the track window contributed by each strip was averaged and is plotted as a function of strip and plane. The top plot shows the gross features, with the central strips contributing approximately $1.8 \%$. The bottom plot is simply a zoom of the top plot and it displays the contributions of the outer strips to the MEU value in more detail. The contribution of the outer strips is due to cross-talk in the MA PMTs. The two strips coloured white in planes 20 and 21 had a bad pedestal reading so were filtered out from the data. 
place the dynodes of each PMT are enabled a few hundred nanoseconds before the test-beam particle arrives. A comparison of data taken with and without the TOF trigger shows that no bias is introduced [68].

\section{MA PMT Cross-talk}

The effect of cross-talk in the MA PMTs has to be considered in two main ways for the relative calibration. Firstly, cross-talk between pixels serving different planes in the detector can cause a track to seem longer than it actually is. Secondly, the cross-talk contributes approximately 5\% "extra" detector response to every event on average.

The effect of cross-talk in distorting the length of a track can clearly be seen in Figure 6.9; this figure shows three histograms: the true event length from MC; the tracked event length; and thirdly, the tracked event length with a cross-talk removal algorithm applied to the end of the track. The cross-talk removal algorithm is relatively straightforward, it works by moving back along the last four planes in a track and removing them until it reaches a plane with a hit greater than 1.7 photoelectrons where it stops. The algorithm is successful in correcting the majority of the tracks affected as can be seen from Figure 6.9. However, this is not important as the effect on the MEU value of employing the cross-talk removal algorithm is negligible $(<0.1 \%)$. This is as expected since the track window technique was designed to use the flatter relativistic rise region of the Bethe-Bloch curve and hence avoid such problems as event length reconstruction.

Cross-talk contributes significantly to the total detector response and thus it must be correctly accounted for in both the Near and Far detectors to avoid introducing any biases. The ability of reconstruction algorithms to reject cross-talk is not straightforward as it depends on the relation between the locations of optical cables on the PMT face and the positions of the strips in the detector; this is because the cross-talk is most likely to occur in the hit pixel's neighbours, which may (or may not) read out scintillator strips that are widely separated in the detector from the position of the real hit. The effect of the optical routing arrangement to pixels on the PMT face is evident at the Calibration detector, as 


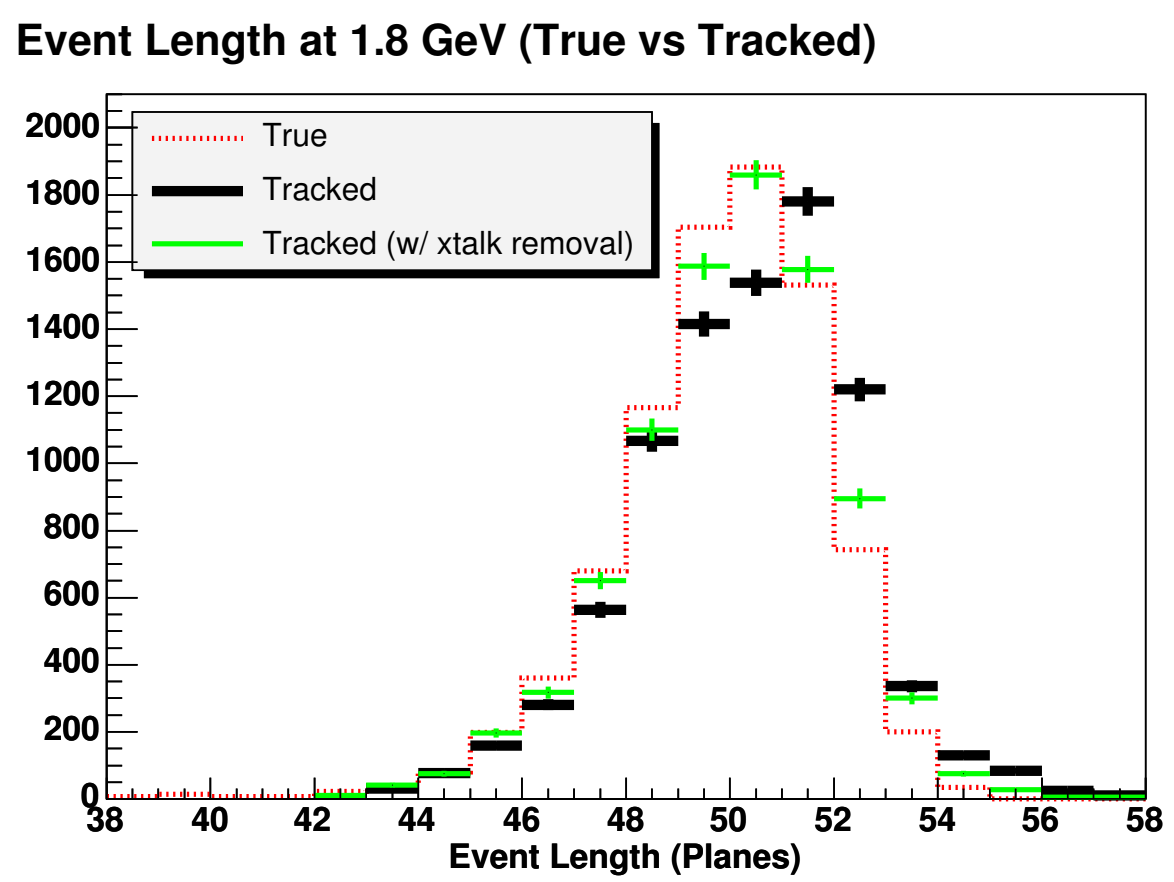

Figure 6.9: Muon event length at $1.8 \mathrm{GeV}$ in $\mathrm{MC}$. The number of planes traversed by $\mathrm{MC}$ muons is shown. The dashed red line shows the true event length while the solid black lines shows the event length measured by the tracking software. The thin solid green lines show the event length after a cross-talk removal algorithm has been applied.

shown in Figure 6.10. The cross-talk occurs in bands on the "wings" of the detector and down the centre. The central cross-talk is directly in the path of the muons and thus often impossible to separate from genuine hits. The arrangement of optical cables on the PMT face differs between the Near and Far detectors thus the performance of cross-talk removal algorithms could introduce a bias between detectors. Another effect is that the fraction of genuine charge that appears as cross-talk differs between the M64 PMTs used in the Near detector and the M16 PMTs used in the Far detector. This could also introduce a bias between detectors.

For the reasons outlined above, it was decided that attempts to remove the cross-talk should only be made for pattern recognition purposes. In performing the relative energy calibration, the measured detector response in the track window includes all the cross-talk that is associated with a scintillator strip (in all detectors). When reconstructing hadronic and electromagnetic showers in the Near detector and Far detector studies will have to be performed to ensure that the reconstruction does not introduce any relative energy scale 
differences.

\section{Pions}

As discussed in section 6.1.1, a potential source of background that is unique to the Calibration detector is from pions contaminating the muon sample and hence changing the energy deposition in the track window. MC simulations show that only a tiny fraction $(\sim 0.25 \%)$ of the pions will pass the event length cut at $1.8 \mathrm{GeV}$ (see Figure 6.2). Given the large ratio of pions to muons produced by the beamline $(\sim 10: 1)$ it is reasonable to predict that approximately $2 \%$ of events in the muon sample will actually be pions. In order to travel the approximately 6 interaction lengths in the detector needed to pass the event length cuts, the pion will have lost a substantial fraction of its energy via ionisation. A pion's ionisation at a given momentum above $\sim 300 \mathrm{MeV}$ is typically a few-percent lower than that of a muon at the same momentum. However, it is possible for the pion to undergo hard scatters along its track and deposit a relatively large amount of energy.

As described in section 6.1.1, muons are selected using a track length cut. Muons are required to stop within a specific range of planes to be included in the event sample (see Table 6.1). The effect of the pion background on the relative calibration is estimated by gradually increasing the contamination of the muon sample and seeing how the MEU value changes. To let more pions into the muon sample the lower track length cut is relaxed. A 1.8 GeV sample of muons is used, which typically have a track length of 46 to 54 planes. Figure 6.11 shows the change in the MEU value as the lower track length cut is reduced, i.e. as more events (muons and pions) are included in the sample. It can be seen that as the lower track length cut is reduced from plane 54 to plane 46 the MEU value changes by $2.5 \%$. Whereas, when the lower track length cut is reduced further, from plane 46 down to plane 35, the MEU value only changes by $0.5 \%$.

The fact that the MEU value changes significantly between planes 46 and 54 is interesting. If the pion background were significant then it would be expected that the MEU value would change significantly between planes 46 and 35, but quite the opposite is observed. 

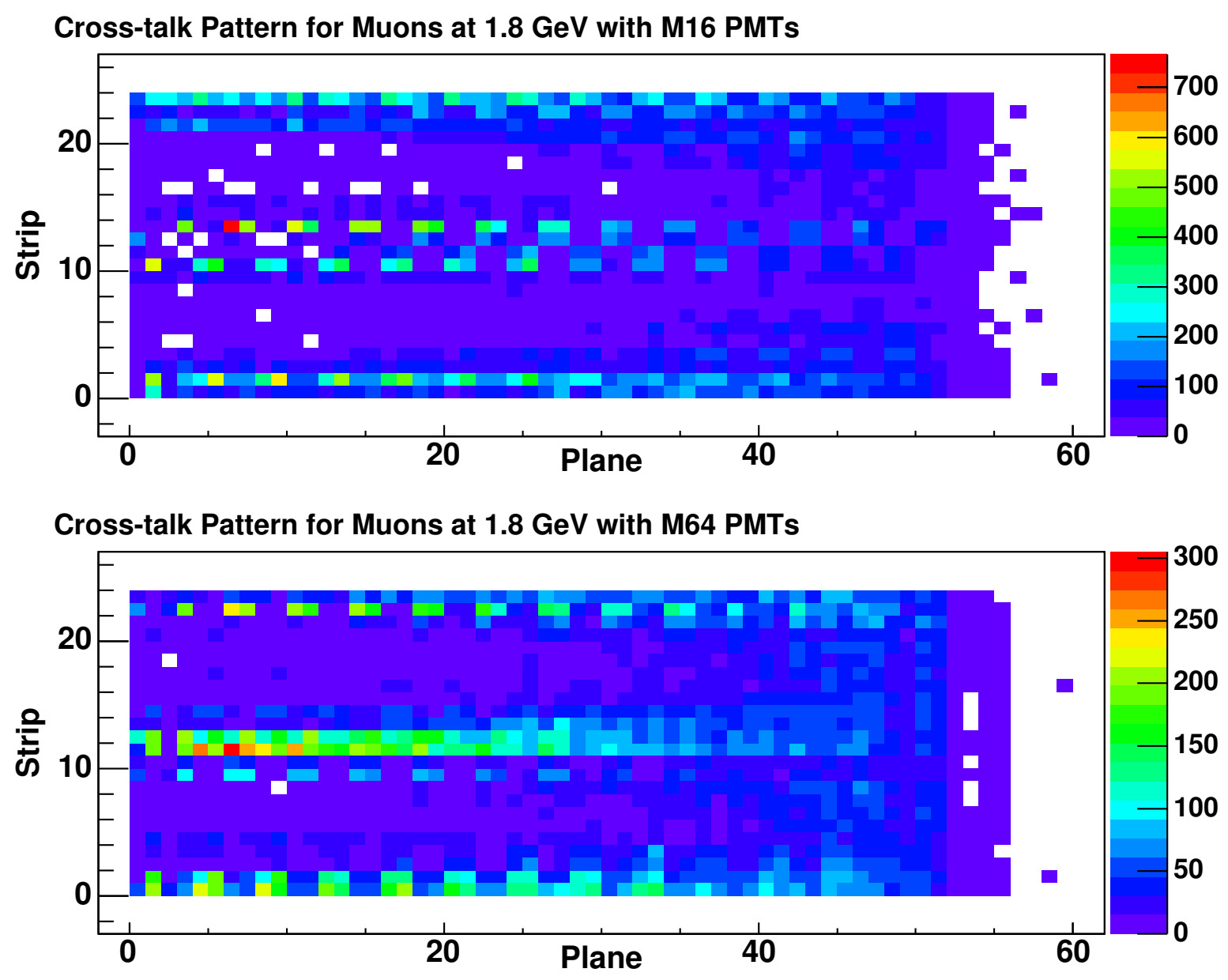

Figure 6.10: Cross-talk pattern for muons at $1.8 \mathrm{GeV}$ with M16 and M64 PMTs. The two plots show the topological pattern of cross-talk that is generated with M16 (top plot) and M64 PMTs (bottom plot) when $1.8 \mathrm{GeV}$ muons traverse the detector. Broadly speaking, three bands of cross-talk are created: at the edge of the detector in two "wings" and also in a central band. The pattern is most prominent when the muons first enter the detector since they are highly collimated but as they come to a stop, around plane 50, multiple scattering causes the pattern to wash out. 


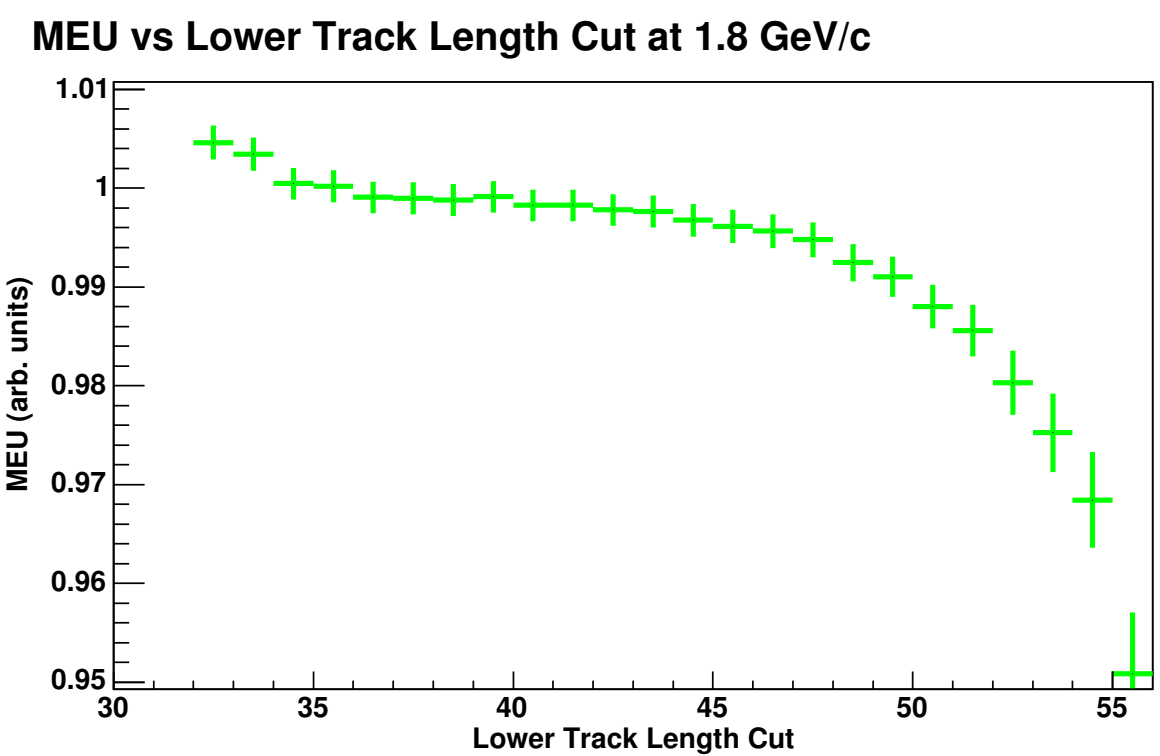

Figure 6.11: MEU vs. lower track length cut at $1.8 \mathrm{GeV}$. The change in the MEU value as more, shorter events are included in the sample is shown. At $1.8 \mathrm{GeV} / \mathrm{c}$ muons typically travel between 46 and 54 planes in the detector.

To understand why the MEU value is lower for muons that have travelled further in the detector it is important to remember that the test-beam produces particles with a small momentum spread (typically 2\%). A muon that has travelled all the way to plane 55 must have deposited less energy upstream in the detector. Conversely, a muon that stops at plane 46 must have deposited more energy upstream in the detector. This explains why the MEU value changes as muons of different ranges but the same initial momentum are included (for more details of this effect see section 6.3.2).

The small, $0.5 \%$ change in the MEU value as the lower track length cut is lowered from plane 46 to 35 suggests that the pion contamination has a small impact on the MEU value. The systematic error on the MEU value due to pions is estimated to be $< \pm 0.25 \%$.

\section{Off-momentum Muons}

The systematic effect of the lower "off-momentum" muons from the test-beam (see section 6.1.1) on the MEU value is evaluated in section 6.3.2 and found to be $\pm 0.25 \%$. 


\section{Intra-detector Calibration Errors and Illumination}

There are a number of potential systematic errors that can occur due to intra-detector calibration and the extent of the detector's illumination, both spatially and in time.

A potential problem unique to the Calibration detector is that the stopping muons only illuminate a thin central portion of the detector whereas the electron and pion showers are much broader. Figure 6.12 shows both the energy deposition for each strip in the track window and the number of times each strip was hit. It can be seen that in the track window the stopping muons between 1.4-2.0 GeV predominantly illuminate strips 9-12 down the centre of the detector between planes 8 and 39. The hits towards the edge of the detector are mostly due to cross-talk in the MA PMTs. The consequence of this limited exposure is that the calibration detector's relative calibration is more sensitive to an accurate strip-tostrip calibration. If the intra-detector calibration were perfect then the response of just one strip to a stopping muon would be sufficient to perform the relative calibration. However, the statistical error on the calibration of each strip is approximately $1.8 \%$ [18][99]. Considering that approximately $(12-9+1) \times(39-8+1)=128$ strips are sampled in the track window, the statistical error that propagates through to the relative calibration is a negligible $1.8 \% / \sqrt{128}=0.16 \%$. However, biases in the strip-to-strip calibration across the detector, say, from front to back could affect the measured response of pion and electron showers with respect to the stopping muon response.

A study of the strip-to-strip calibration constants has been done using the PS muons (see section 6.1.1). The major advantage of using PS muons is that they all travel horizontally and more or less in the same direction, which is in strong contrast to the wide angular spectrum of the cosmic muons. The straightforward nature of analysing the PS muons significantly reduces the potential biases of the complicated path length reconstruction and zero correction procedure needed to produce a strip-to-strip calibration using the cosmic muons. Calibration constants computed using the two muon samples have been compared on a strip by strip basis [99]. The statistical error on the calibration constant for each stripend, using either source of muons, is approximately $1.8 \%$ so the error on the difference is expected 

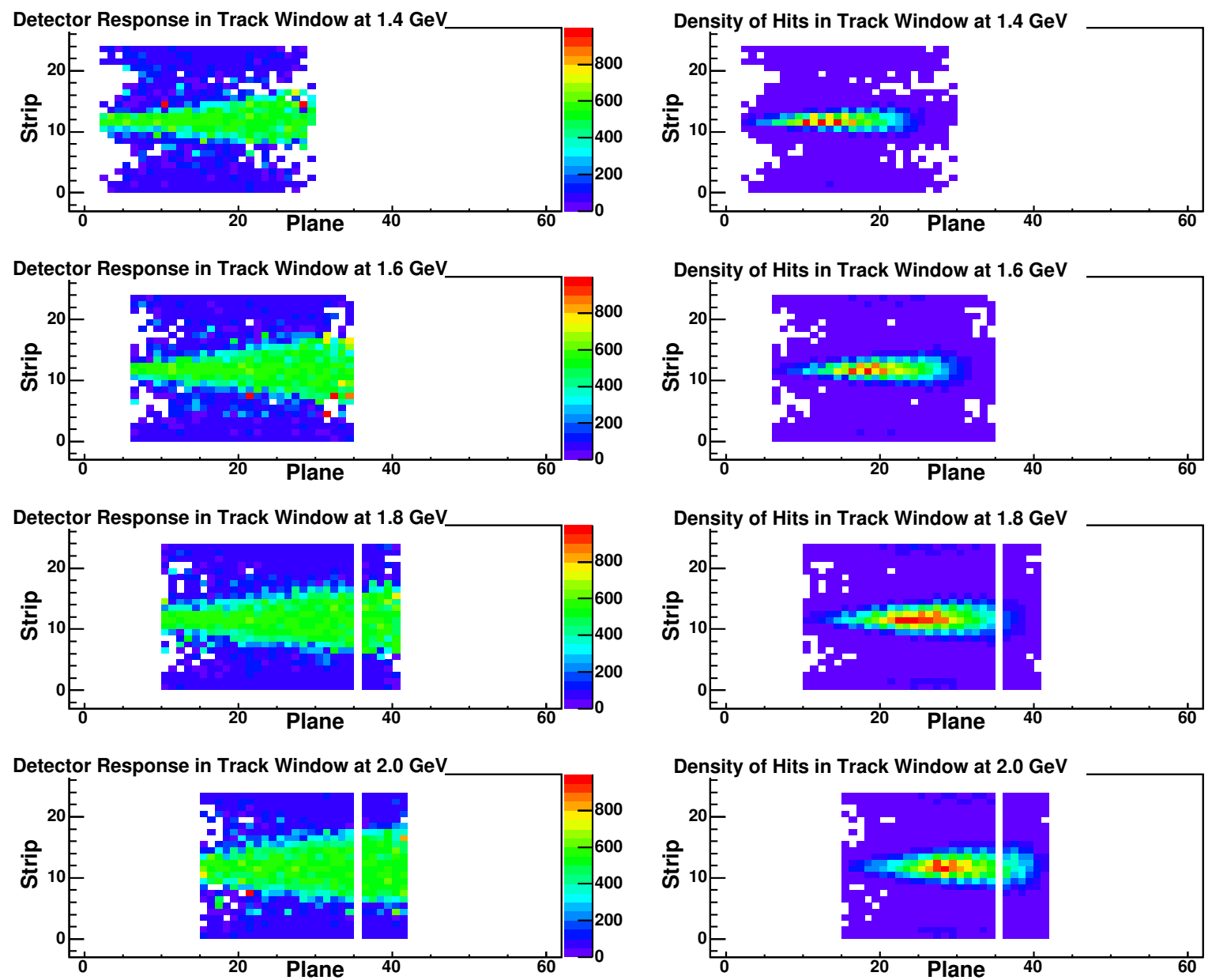

Figure 6.12: Detector regions used in the track window at different energies. Two different strip vs. plane maps are shown: the left hand column shows the detector response in SigMaps and the right hand column shows the density of hits in the detector. It is clear to see that only a central band of strips have a significant contribution to the measured detector response. The hits towards the edge of the detector are caused mostly by cross-talk in the MA PMTs. Plane 35 was not reading out for the 2002 runs hence it has no entries in the plots. 
to be $\sqrt{0.018^{2}+0.018^{2}}=2.5 \%$. However, an error of $2.8 \%$ is observed thus suggesting the presence of a $1.2 \%$ systematic error $\left(\sqrt{0.028^{2}-0.018^{2}-0.018^{2}}=1.2 \%\right)$. Such a value is a conservative estimate but since the region of the detector sampled by the stopping muons is not the same as that sampled by the electrons a cautious approach has been taken.

As mentioned at the start of this section, the Calibration detector's response may change over time. In order to evaluate the magnitude of this effect, the detector response to stopping muons has been evaluated separately for the 4 different energies $(1.4,1.6,1.8,2.0 \mathrm{GeV} / \mathrm{c})$. The data from the T7 2003 run period has been used since there are not enough data points in the other two run periods. The advantage of considering each energy separately is that the effect of errors due to the strip-to-strip calibration, pion contamination, lower "off-momentum" muons and so on will be constant. Figure 6.13 is a very similar plot to Figure 6.7 except the points have been rearranged such that they are increasing in energy along the x-axis. The blue triangles, green squares, turquoise triangles and purple circles show the 1.4, 1.6, 1.8 and $2.0 \mathrm{GeV} / \mathrm{c}$ runs respectively. A fit to a constant has been made for each set of points and the results are shown by the black lines. The sum of the $\chi^{2} / n d f$ for the 4 independent fits gives a total of $64 / 20=3.2$, which is slightly worse than the fit of a single constant to all 24 points $\left(\chi^{2} / n d f=67 / 23=2.9\right)$. This suggests that there is no correlation of the MEU value in this run period with the test-beam momentum. As discussed in section 6.1 .2 an additional error of $0.5 \%$ added in quadrature with the statistical errors reduces the $\chi^{2} / n d f$ to unity. Since there is no variation with energy it is reasonable to take this error as the error due to temporal variations.

\subsubsection{A Cross-check against the Test-beam Energy}

One of the great advantages of the Calibration detector data is that the measurements of the relative calorimetric response in the different run periods can be cross-checked against the known test-beam energy. For this thesis, electrons from the test-beam are used since they are the most reliably identified and selected.

The detector response to electrons for each run in the different run periods is determined. 


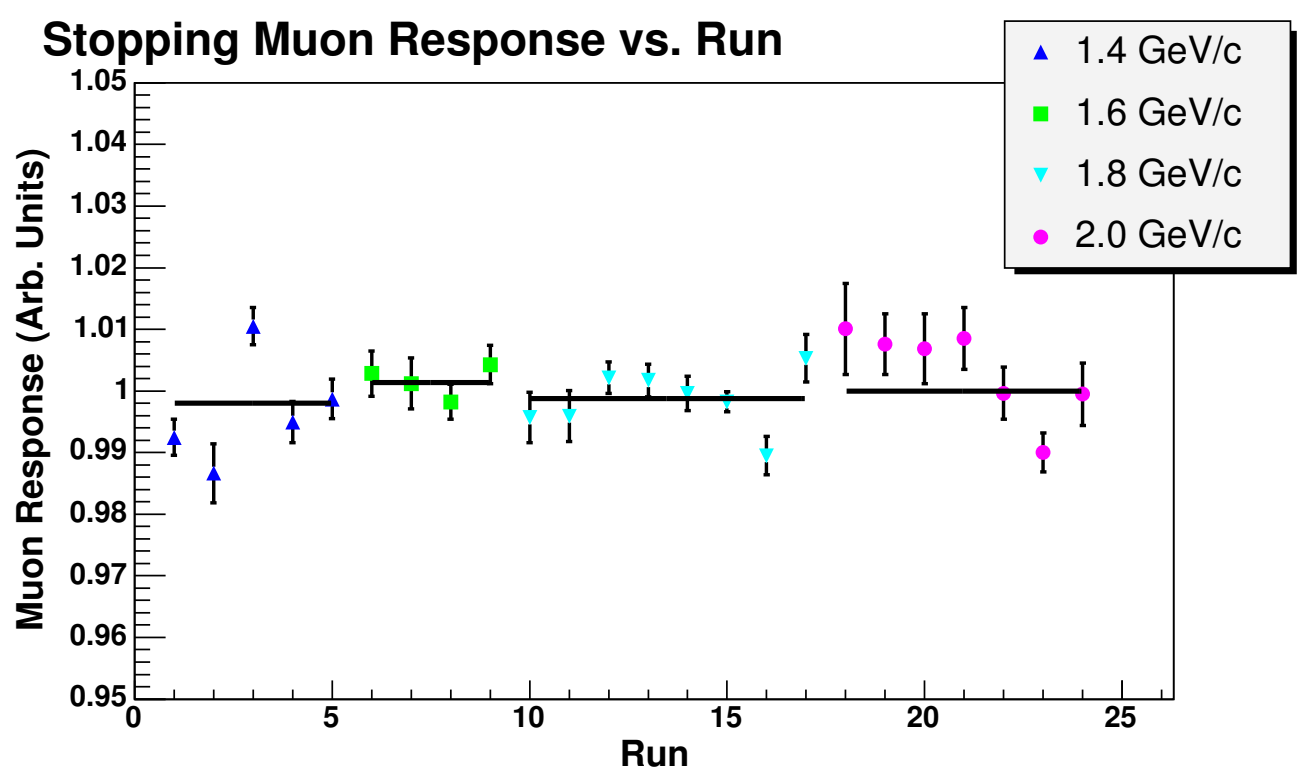

Figure 6.13: Stopping muon response vs run $(1.4-2.0 \mathrm{GeV} / \mathrm{c})$. The blue triangles, green squares, turquoise triangles and purple circles show the 1.4, 1.6, 1.8 and $2.0 \mathrm{GeV} / \mathrm{c}$ runs respectively. A fit to a constant has been made for each set of points and the results are shown by the black lines

The energy of the electrons is known from the test-beam so it is straightforward to determine the detector response per $\mathrm{GeV}$ of electron energy for each run. Figure 6.14 shows the fully calibrated electron response per $\mathrm{GeV}$ for many runs. It can be seen that the fully calibrated electron response per $\mathrm{GeV}$ agrees to better than $2 \%$ between the different run periods even though the detector response in SigMap units differs by as much as $10 \%$.

Table 6.4 lists the differences between the average fully calibrated electron response per $\mathrm{GeV}$ in the different run periods. It can be seen that the difference in electron response between T11 2002 and T7 2002 is close to consistent with zero. The largest disagreements are between the two 2002 run periods and the T7 2003 run period. It has been shown that the strip-to-strip calibration for the T7 2003 run period varies slightly along the z-axis of the detector [76]. The track window technique samples the middle of the detector whereas the electrons are sampled at the front of the detector, hence a bias could occur. In addition, jumps in the detector response in SigMap units of up to 5\% from plane to plane have been observed using muons in the T7 2003 run period. Such jumps affect the response of electrons of different energies differently and could explain some of the scatter in the electron response shown in Figure 6.14. 


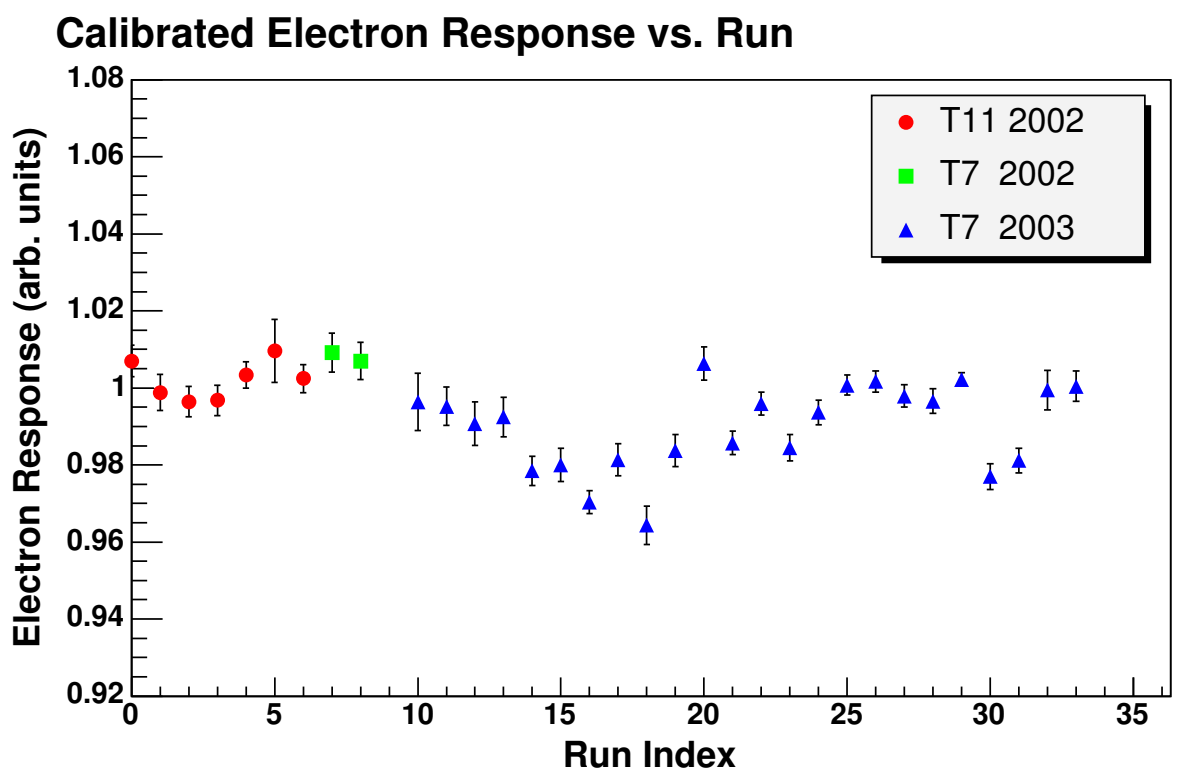

Figure 6.14: Calibrated electron response vs. run index. The electron response per GeV is shown after full calibration for many runs. The red circles, green squares and blue triangles show the electron response for runs in the T11 2002, T7 2002 and T7 2003 run periods respectively. The error bars show the combined statistical error on the means of the muon and electron sample. The point for run index 9 is missing from the plot since no electron events were recorded during that run (the electrons were not triggered on in order to reduce the size of the raw data files).

In order to assess the size of the additional source of variability causing the scatter of the points within each run period in Figure 6.14 an error is added in quadrature to the statistical errors until the $\chi^{2} / n d f$ is unity. A $0.2 \%$ and $1.0 \%$ error is required for the T11 2002 and T7 2003 run periods respectively. No additional error is required for T7 2002.

To study the reproducibility of the test-beam momentum regular $1 \mathrm{GeV} / \mathrm{c}$ runs were taken in the T7 2003 run period. It was found that the combined calibrated detector response plus test-beam momentum was constant to $\pm 0.8 \%$ [70]. Similarly, by comparing the variance at all the different momenta in T7 2003 it was found that the measured response was constant to $0.8 \% \pm 0.8 \%$. This variance is roughly consistent with the additional $1 \%$ error required to reduce the $\chi^{2} / n d f$ to unity. Although a reasonable estimate of the testbeam momentum reproducibility has been obtained, the error on the absolute value of the test-beam momentum is not well understood. The positions of the test-beam magnets and the accuracy to which their magnetic fields are calibrated affect the absolute momentum. Enquiries of the PS staff have been made but without further studies and/or measurements 


$\begin{array}{cc}\text { Run periods } & \begin{array}{c}\text { Difference in } \\ \text { electron response }\end{array} \\ \text { T11 2002- T7 2002 } & -0.6 \% \pm 0.5 \% \\ \text { T11 2002- T7 2003 } & +1.2 \% \pm 0.3 \% \\ \text { T7 2002- T7 2003 } & +1.8 \% \pm 0.4 \%\end{array}$

Table 6.4: Differences in fully calibrated electron response per $\mathrm{GeV}$ in the three Calibration detector run periods.

of the test-beam line components the error on the absolute test-beam momentum is not felt to be well known. Comparisons of the range of test-beam muons with that from MC reveals a $\sim 3 \%$ discrepancy, the source of which is not understood [68] except that it may be a due to an offset in the test-beam momentum. Further studies of the muon range and more detailed simulations of muon production in the test-beam line will need to undertaken to improve the knowledge of the error on the absolute test-beam momentum.

It is interesting to note that this cross-check also applies in reverse. If the beam momentum was erroneously high in one run period w.r.t. another then that would introduce differences in the fully calibrated electron response per $\mathrm{GeV}$. It is possible, but considered unlikely, that the beam momentum could be different by the observed $1.8 \%$ between $\mathrm{T} 7$ 2002 and T7 2003. The strip-to-strip calibration is considered the more likely culprit to contribute a significant fraction of the difference since the error on the strip-to-strip calibration is $\pm 1.2 \%$.

\subsubsection{Summary of Errors and Conclusions}

The statistical errors at the Calibration detector are in the worst case $0.3 \%$. A summary of the systematic errors (described in section 6.1.3) on the Calibration detector MEU value is given in Table 6.5. Adding the statistical and systematic errors in quadrature gives a total error of $1.4 \%$. Thus, it can be seen that the error on the strip-to-strip calibration dominates the total error.

The cross-check of the measured detector response with electrons of known energy from the test-beam (see section 6.1.4) reveals that the agreement is better than $2 \%$ for all three run 
Cause of Error

No Fibre Attenuation Calibration

Strip-to-strip calibration

Temporal fluctuations

Pion background

Off-momentum muons
Error value

$\pm 0.2 \%$

$\pm 1.2 \%$

$\pm 0.5 \%$

$\pm 0.25 \%$

$\pm 0.25 \%$

Table 6.5: Systematic errors on the Calibration detector MEU value.

periods. Although it important to note that a detailed breakdown shows that the difference between the T11 2002 and T7 2002 is consistent with zero. The largest differences (1.2\% and $1.8 \%$ ) are between the two 2002 run periods and the T7 2003 run period. It is believed that this is most likely caused by the strip-to-strip calibration since that has the largest systematic error.

In conclusion, an accurate measurement of the relative calorimetric energy response of the three Calibration detector run periods has been accomplished using the track window technique. The total error on the measurement is $1.4 \%$, which is well below the $2 \%$ relative calibration target. In addition, the results have been independently confirmed by showing the consistency of the test-beam energy compared with the measured detector response.

\subsection{The Far Detector}

At the Far detector, stopping cosmic muons are used to do the relative calibration. Typically, these cosmic muons have an energy of between 1.5 and $4 \mathrm{GeV}$ when they enter the detector. The Far detector's deep location means that it experiences a relatively low rate of cosmic muons, approximately $1 \mathrm{~Hz}$, compared to the other two detectors. Around 1/300 of the cosmic muons actually stop in the detector's fiducial volume, thus giving a rate of approximately $0.003 \mathrm{~Hz}$. A sample of approximately 80000 stopping muons, recorded between August 2003 and October 2004, is used to do the calibration presented in this thesis.

In order to make comparisons with the MC, a sample of $\sim 40000 \mathrm{MC}$ cosmic stopping muons is used (a larger sample was not available at the time of writing and the statistical benefits of such a sample were not necessary for this thesis). The stopping cosmic muons 
were selected from a cosmic muon sample generated for the atmospheric neutrino analysis. The angular spectrum at different energies has been established in previous work [100]. Detailed studies of how well the MC muon sample matches the data have been performed in $[100,101]$. In addition, some new aspects the MC/data comparison that are important for the measurement of calorimetric energy scale are made in this section.

\subsubsection{Stopping Muon Reconstruction}

\section{Pre-selection}

The pre-selection process developed for the atmospheric neutrino analysis $[101,102]$ is used to determine for which runs the detector was fully operational. Any runs during which the high-voltage is not at its nominal setting are discarded. The Detector Control System (DCS) monitors the current applied to the magnetic coil and this is required to be $>70 \mathrm{kA}$ or the run is discarded.

\section{Reconstruction Software}

Two independent sets of reconstruction software are used to track stopping muons in the Far detector. The results obtained with each set of software are the same to within statistical error. The two sets of reconstruction software used are the Standard Reconstruction (SR) software and the "AtNu" software, which was developed with a focus on the atmospheric neutrino analysis. Both sets of software run in the MINOS offline-software framework [78].

The reconstruction software was used principally to find single track events. Information about the presence of a "shower" (or not) in the event is not used. The track fitting procedure in the reconstruction that attempts to determine the muon's momentum from curvature is also not used.

Once a track is found the information on which strips form the track is used in a customised fitting procedure. Simple straight lines are fit to groups of 3-5 planes in order to 
determine the 3-dimensional position and path length of the muon through each plane. The details of the custom fitting procedure are described in section 5.3.

\subsubsection{Stopping Muon Selection and Backgrounds}

Selection of stopping cosmic muons at the Far detector is centred around determining if the event is partially contained (PC). The vertex of each event is required to be outside a fiducial volume and the end point within the fiducial volume. Event quality cuts are made to reject through-going cosmic muons that appear partially contained. Additional cuts on the performance of detector components in the region where the event occurred are also made.

\section{Fiducial volume definition}

A conservative fiducial volume was defined for this analysis. It is essential to reject the background from high energy cosmic muons that can appear partially contained. In addition, the analysis is not statistically limited so events can be sacrificed in order to obtain a pure sample. The fiducial volume is defined as:

- Plane cuts on super-module ends:

Super-module 1: $5<$ Plane $<244$

Super-module 2: $254<$ Plane $<481$

- Radius cut within the super-modules:

Radius $<3.0 \mathrm{~m}$

In the case that a muon enters a super-module via the end, rather than the side, the radius of the muon vertex is not required to be $>3 \mathrm{~m}$.

\section{Event selection and quality cuts}

In this section the cuts are first listed in a concise form and then more detail is given below. A single track is required to found by the offline software and the following cuts are applied: 
- Event selection cuts

1. Track $>20$ planes.

2. Muon direction from timing and geometry information must agree that the event was downward going.

3. Track vertex outside fiducial volume.

4. Track end point inside fiducial volume.

5. Track does not cross the super-module boundary.

6. Track is reconstructed entirely within the detector volume: $|x|<4.1 \mathrm{~m}$ and $|y|<4.1 \mathrm{~m}$ at all points along the track.

The requirement that the timing and geometry information agree ensures that the vertex end of the track is always correctly identified. The approximately 100 neutrino-induced upward going muons expected in the sample (of which only a fraction actually stop in the detector) are thus sacrificed in order to ensure very high purity in vertex determination.

Approximately $5 \%$ of the PC muons are reconstructed as crossing the $1.2 \mathrm{~m}$ gap between super-modules. These events are rejected since the additional reconstruction difficulties do not justify the small increase in the number of events. The presence of tracks outside the detector volume is used to flag events where the reconstruction has not worked correctly and thus reject these events.

- Event quality cuts

1. Track is away from magnetic coil hole (radius $>0.5 \mathrm{~m}$ ).

2. Track end points in the two views must agree to 5 planes.

3. No more than 500 SigCors ( 1 MEU) allowed beyond the end point of the track.

4. The sum of the detector response in planes with no tracked hits in the last 7 planes of the track $<500$ SigCors $(\sim 1 \mathrm{MEU})$.

5. Track projection along the z-axis $>0.5 \mathrm{~m}$ from the edge of the detector. 
6. Track is long enough for a complete "track window".

The event quality cuts are applied sequentially to each event in the order listed above. One way that through-going muons can appear partially contained is by exiting the detector via the magnetic-coil hole. Thus, the fiducial volume excludes the magnetic-coil hole by requiring that the track radius is $>0.5 \mathrm{~m}$ at all points along the track.

Event quality cut 2 requires that the ends of the track in the U-view and the $\mathrm{V}$-view agree to 5 planes. A gap of 3 planes corresponds to just one hit being missing at the end of the track in one view, thus a gap of 5 planes means that 2 hits are missing at the end of the track in one view. The track finding is performed independently on the two views thus it is possible that the two views do not align properly.

If the track finding algorithm fails to reconstruct the whole track because, say, of a gap in the track or sharp curvature at the end of the track then these events need to be rejected. Event quality cut 3 requires that the total signal beyond the track end point is $<500$ SigCors (the calibrated ADC value obtained after the strip-to-strip calibration has been applied). A value of 500 SigCors is chosen since that is close to $1 \mathrm{MEU}$ and hence roughly equivalent to what is expected from a muon passing through 1 plane.

Determining if a steep muon stopped in the fiducial volume is particularly sensitive to accurate reconstruction of the track end. Event quality cut 4 requires that the sum of the detector response in any planes with zero tracked hits in the last 7 planes is $<500$ SigCors. The presence of planes with no tracked hits but a significant signal at the end of a muon's path is often caused by mis-reconstruction. Failure to include genuine hits as part of the muon's track leads to an erroneous 3-dimensional position that can place a through-going muon inside the fiducial volume.

The MINOS detectors are designed to maximise the acceptance and containment of muons from beam neutrino events. Steep downward going cosmic muons are able to exit the fiducial volume without leaving a detectable signal. The muons can exit in this way by travelling parallel to the planes in the steel or in the $2.4 \mathrm{~cm}$ air gap. An effective solution 
to this problem is to consider the projection of the muon track out to the edges of the detector $^{1}$. In particular, the z-component of the projection is used as this is a good measure of the amount of material that the muon would have had to "escape" through. Event quality cut 5 uses the last five planes of the muon track to calculate the z-component of the projection and requires it to be $>0.5 \mathrm{~m}$. In the case that the muon was projected at one of the super-module ends the cut was not applied since the 5 plane cut made on the super-module ends is adequate.

The 6th event quality cut simply requires that the muon traversed enough material through the detector for a complete track window $(95+83=178 \mathrm{~cm})$ to be obtained.

Figure 6.15 shows the distributions of the quantities used to make the event quality cuts and thus the effectiveness of the different cuts can be determined. For each cut quantity, the distribution after the event selection cuts but before any event quality cuts is shown in black, the distribution resulting from making all but that particular event quality cut is shown in red, and lastly the distribution after making all the event quality cuts is shown in blue. The effectiveness of cutting events out that are close to the coil hole is particularly noticeable (see top left plot). The remaining cuts reject relatively rare events to produce the final pure sample, as can be seen from the distributions.

\section{Detector performance cuts}

In addition to the cuts on the reconstructed quantities it is also necessary to consider the performance of the detector components in the region where the stopping muon events occurred.

The FarDetDataQuality software package [78] is used make the detector performance cuts. To determine the regions of the detector that are potentially not performing correctly this package analyses several sources of data: the time of every LI flash; the summaries of the one-second-average trigger rate of every VA electronics chip; and the hits that occurred in the detector in the previous $30 \mu \mathrm{s}$. Using this information the VA chips are labelled as

\footnotetext{
${ }^{1}$ This projection cut is often referred to as the "trace" cut in the atmospheric neutrino analysis.
} 

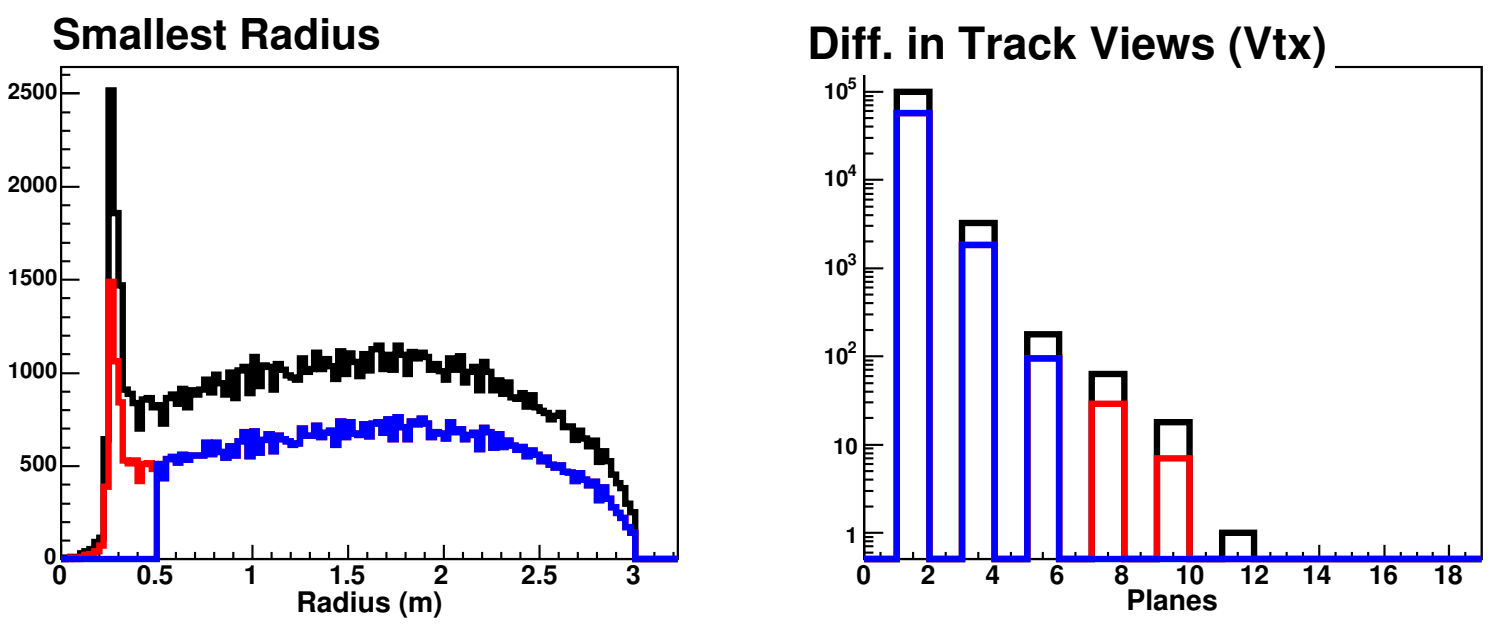

\section{Signal Beyond Track End}

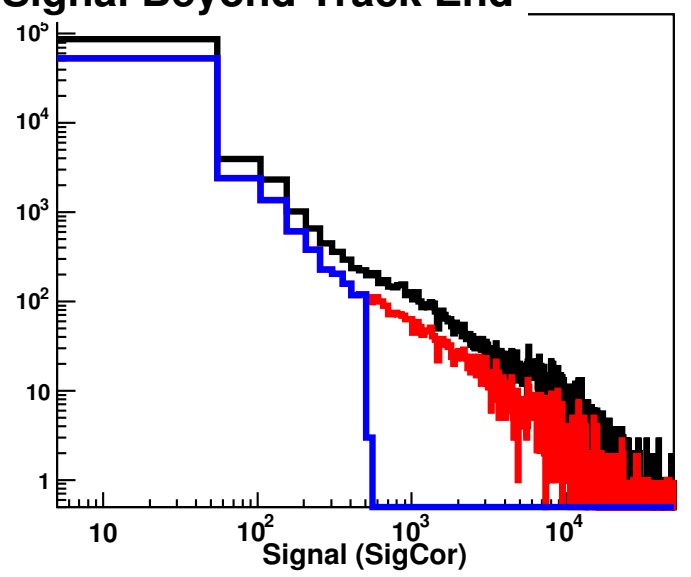

Track Projection (Trace Z)

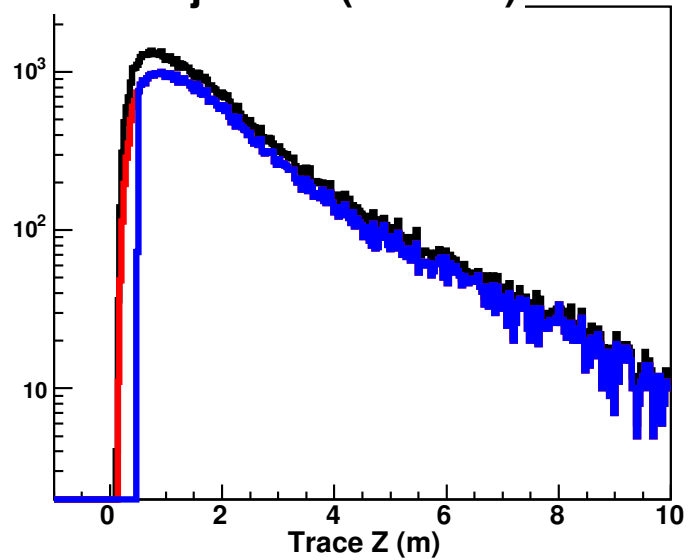

Signal in Trk End Gaps
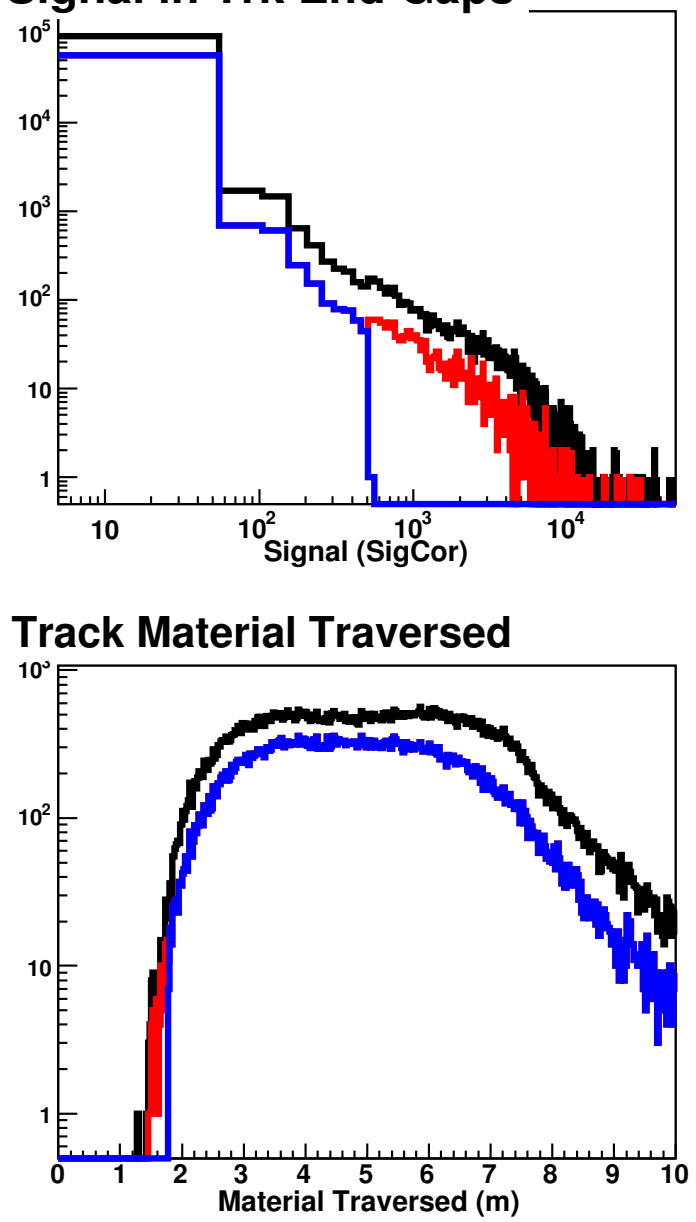

Figure 6.15: Event quality cuts at the Far detector. The distributions of the quantities used to made the event quality cuts are shown. The top left and right plots show event quality cuts number 1 and 2 respectively. The middle plots show cuts 3 and 4 . The bottom plots show cuts 5 and 6 . For each cut quantity, the distribution after the event selection cuts but before any event quality cuts is shown in black, the distribution resulting from making all but that particular event quality cut is shown in red, and lastly the distribution after making all the event quality cuts is shown in blue. 
either good, busy, cold or hot. A busy chip is one that is still digitising a hit that occurred before the current event. A cold chip has an average rate lower than $50 \mathrm{~Hz}$ and a hot chip has a rate higher than $2.5 \mathrm{kHz}$ (the average chip has a rate of approximately $350 \mathrm{~Hz}$ ). The cold chips are typically caused by a malfunctioning VA chip or by a PMT base that has failed. Hot chips can occur because of light leaks into the fibre optic cables.

- Detector performance (data quality) cuts

1. No busy, cold or hot VA chips in the track window.

2. No busy or cold VA chips in the first 2 planes beyond the track end.

3. Event is more than $35 \mu s$ away from any previous LI flash.

4. Event is more than $100 \mu s$ away from the edge of each DAQ time frame.

Detector performance cut 1 requires that there are no busy, cold or hot chips in the track window since that can affect the observed detector response. The presence of dead chips (busy or cold) in an event can also affect whether or not it appears partially contained. Thus, the 2nd detector performance cut requires that there are no dead chips in the first 2 planes beyond the apparent end of the muon track. The effect of the dead chips is evaluated in section 6.2.4.

Occasional disruptions of the LI triggering system mean that flashes of light from the LEDs can appear in the data stream. In order to remove this potential background, detector performance cut 3 requires that each stopping muon event occurs $>35 \mu s$ after any LI flash. The 4th detector performance cut requires that the event did not occur within $100 \mu s$ (only $10^{-4}$ of total live-time) of the edge of a DAQ time frame (a 1-second unit of data). The structure of the DAQ trigger processing stage is such that each time frame is processed independently. With the early DAQ implementation it was possible for an event to get split across the boundary between time frames (although a solution to this problem has since been implemented). It is necessary to remove these split-events since they will have hit strips missing. 


\section{Backgrounds}

Potentially, the dominant background to the cosmic stopping muon sample at the Far detector is actually other cosmic muons. The average energy of a cosmic muon at the Far detector is of order $200 \mathrm{GeV}$ and only around 1/200 stop in the detector. To achieve a signal to background ratio of $100 / 1$ the efficiency of background rejection has to be approximately $99.995 \%$.

The tight fiducial volume and event quality cuts defined above successfully remove all but a tiny fraction of the high energy cosmic muons. A MC study of background rejection, using the full spectrum of cosmic muons showed that the background from high energy cosmic muons was only $0.1 \%$.

\subsubsection{Results}

A relative energy calibration has been performed for the Far detector using data taken between August 2003, shortly after the detector construction was completed, and October 2004. The results stated in this section are given in "SigMap" units, which is the unit of detector response after the strip-to-strip and fibre attenuation calibrations have been applied.

The MEU value for the Far detector, calculated using equation 5.8, is found to be $504.0 \pm$ 0.4 (stat.) SigMaps. However, there are two relatively small systematic effects that have to be corrected for. These two systematics effects are explained in detail in section 6.2.4. The correction for the angular dependence of the MEU value is determined to be $-0.5 \%$ and the correction for the spatial variations in detector response is determined to be $+0.6 \%$. Thus, after these two corrections the MEU value for the Far detector is

$$
(504.5 \pm 0.4 \text { (stat.) } \pm 4.5 \text { (syst.)) SigMaps. }
$$

The systematic error on the MEU number quoted here is evaluated and described in detail in section 6.2.4. 


\subsubsection{Sources of Systematic Error}

\section{Temporal Drifts in Detector Response}

The LI drift calibration and the through-going muon drift calibration are not available at the time of writing so temporal fluctuations in the detector response have not been calibrated out. The top plot in Figure 6.16 shows how the average detector response in SigMaps drifts over the 15 months shown. The middle plot shows the average temperature in the detector cavern over the same time period. The correlation between detector response and temperature can be clearly seen in the bottom plot.

It is expected that the LI drift calibration will remove the majority of the drift in the detector response. The fractional drift of the combined PMTs and electronics has been measured to be $0.008 \pm 0.002$ per ${ }^{\circ} \mathrm{C}[103,104]$. The straight line fit to the bottom plot in Figure 6.16 gives the fractional drift of the entire detector readout system to be $0.010 \pm 0.001$ per ${ }^{\circ} \mathrm{C}$. This value agrees with the previously measured value for the PMTs and electronics to within statistical errors.

The effect of these temporal drifts on the neutrino measurements depends on the details of such a measurement. If, say, only 1 neutrino event was found in the 15 month period then its would have a conservative error on its calorimetric energy scale of $\pm 2 \%$, since the maximum variations are $\sim 4 \%$. However, in the case of the atmospheric neutrino analysis approximately 150 neutrino events have been found in the period considered. These events occurred at random throughout the period and are thus distributed relatively uniformly, in fact the live-time is identical since the same pre-selection process was used. The effect of the temporal variations on this uniform distribution is a slightly worse energy resolution and an error of $\sqrt{150 \times 2^{2}} / 150=0.2 \%$ on the calorimetric energy scale. Thus, although the temporal variations have the potential to introduce significant systematic errors, when reasonable numbers of events (e.g. 150) are used the systematic errors on each event average out. 
Detector Response vs. Time

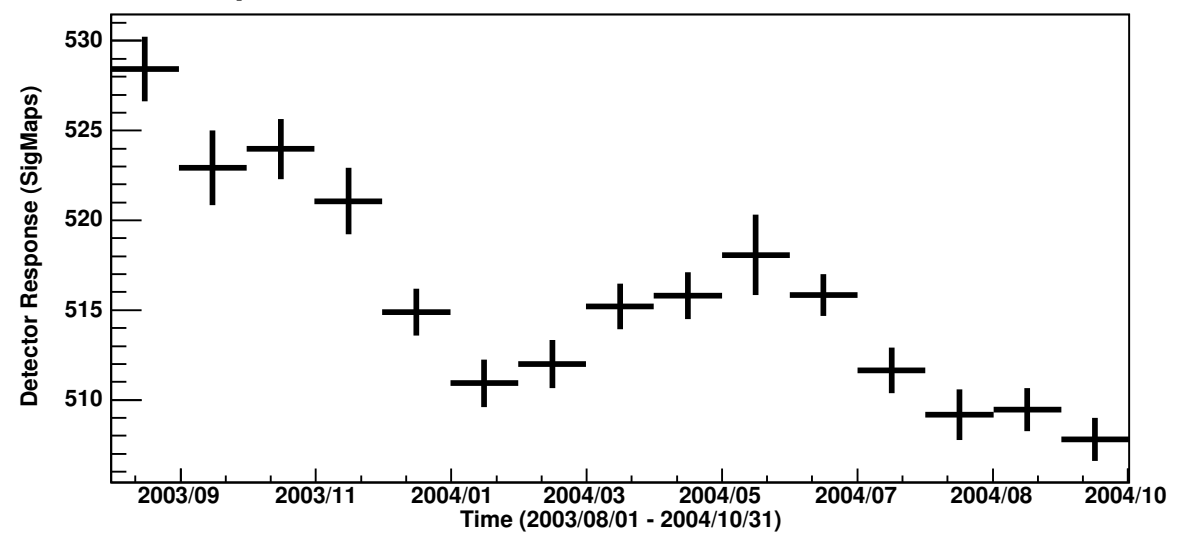

Temperature vs. Time
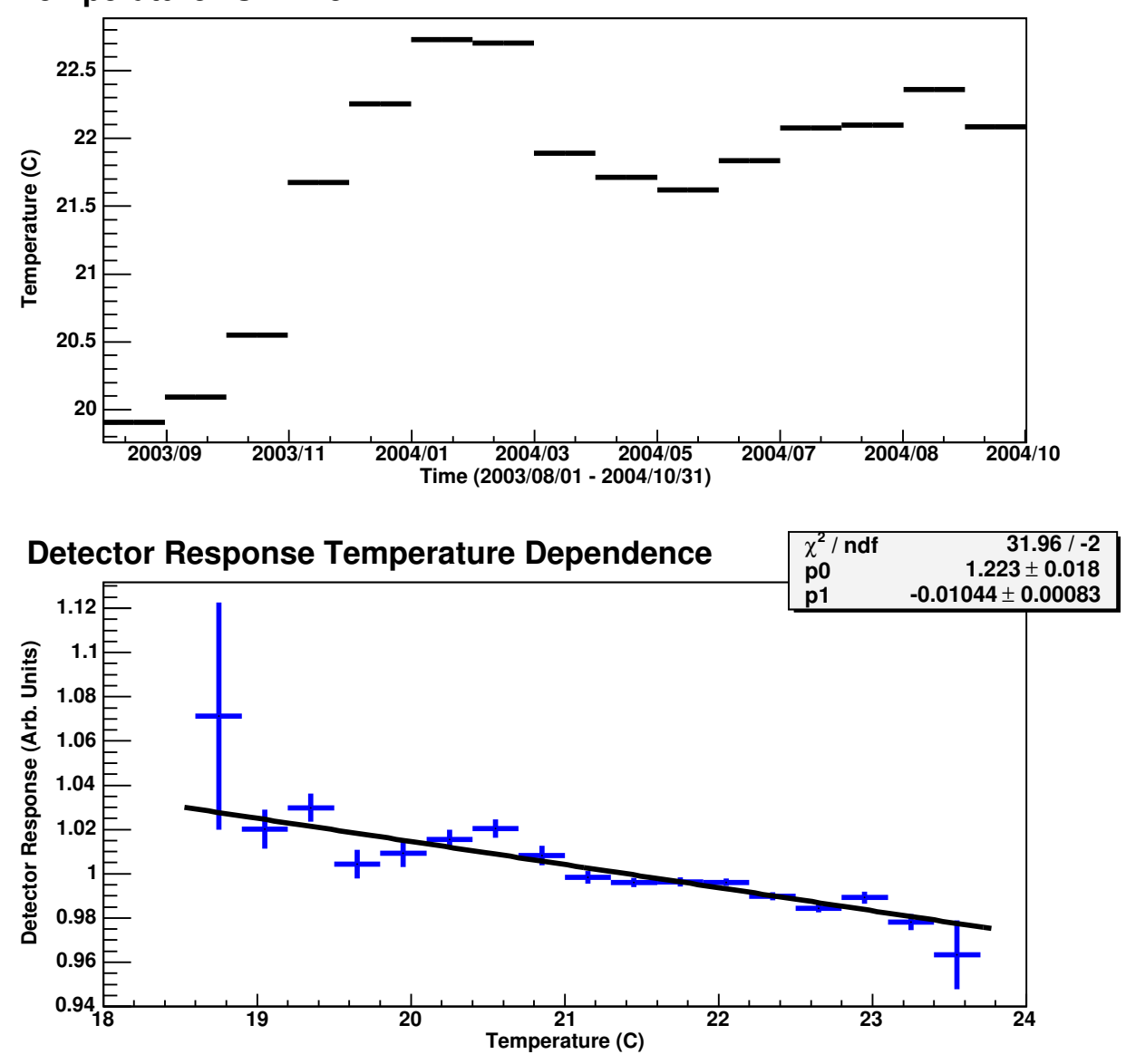

Figure 6.16: Detector response over time. The top plot shows the variation of the detector response over a period of 15 months. The middle plot shows the temperature variation over the same period. The bottom plot shows how the detector response correlates with the temperature. 


\section{Spatial Variations in Detector Response}

Before an accurate relative energy calibration can be achieved, all spatial variations in the detector response have to be calibrated out. However, at the time of writing it is believed likely that significant problems exist with the strip-to-strip calibration and/or the attenuation calibration at the Far detector. No such problems are observed in MC data, thus either the detector is not simulated properly or the apparent calibration problems are genuine. For the sake of simplicity in presenting the results below, it will be assumed that erroneous calibration is the cause of the problems. A discussion of the possible causes of the problems and the resulting systematic error takes place at the end of this section.

Stopping muons can be used as a standard candle to illuminate different parts of the detector and thus checks can be made to see if the response is uniform throughout. In order to ensure that the muons have the same $\mathrm{dE} / \mathrm{dx}$ throughout the detector only hits in the track window are used. However, since the $\mathrm{dE} / \mathrm{dx}$ of the muon changes by $\sim 6 \%$ across the track window an additional correction is required. The $\mathrm{dE} / \mathrm{dx}$ changes approximately linearly thus it is straightforward to correct the measured energy deposition depending on where in the track window the muon was when it passed through a given plane. In the $y$ vs. $x$ plots of the detector response below, an entry to the $2 \mathrm{D}$ profile histogram is made for each plane in the track window at the $x-y$ position determined by the track finder. The colour scale represents the average detector response at each $x-y$ location.

As a first look, it is revealing to examine the detector response for intermediate steps in the calibration chain as well as the final result. Figure 6.17 shows the response of the two stripends in $\mathrm{U}$-view planes for various calibration stages as a function of $x$ and $y$ coordinates. The top pair of plots show the detector response in raw ADCs for the stripends on the east and west sides of the detector. The middle pair of plots show the detector response in SigCors, i.e. after the strip-to-strip calibration has been applied. The bottom pair of plots show the detector response in SigMaps, i.e. after the attenuation calibration has been applied. It can be seen that in calibrating from ADCs to SigCors the detector response becomes symmetric about the line through the centre of all the strips (that is, the line perpendicular to the 
black line showing the orientation of the strips). Once the strip-to-strip calibration has been applied then the centre of each strip should have an identical response, while the effect of the attenuation along the length of the strips is still clearly visible. It can be seen from the bottom pair of plots that applying the attenuation calibration significantly flattens the detector response as expected. However, the coarseness of the colour scale means that any differences less than $\sim 10 \%$ cannot be observed in this plot.

Figure 6.18 shows in detail the detector response after the full calibration chain has been applied (SigMap units). The detector response is shown as a function of $x$ and $y$ for both stripends of both plane views. The top two plots are just a zoom of the bottom pair of plots in Figure 6.17. The enhanced granularity of the colour scale afforded by the zoom allows resolution on the scale of $\sim 2 \%$. The detector response as measured by each stripend is expected to be uniform. However, patches of red and blue can be clearly seen. For example, in the top left plot of Figure 6.18 there is a red patch at coordinates $(x, y)=(-2.5,1)$, which contrasts with a blue patch at coordinates $(x, y)=(2.5,1)$. These contrasts in colour amount to $\sim 15 \%$ differences in detector response.

To further understand the significant differences in detector response shown in Figure 6.18 it is necessary to consider the geometry of the detector. The side of the detector read out is shown by the purple line running along the detector outline in each plot. Scintillation light produced close to the purple lines has the least distance to travel to the PMTs. Furthermore, the strips that are read out at the top of the detector have the longest clear fibres. It can be seen in Figure 6.18 that the detector response varies along the length of a strip (parallel to the black line across the detector shown in the plots). In addition, the strips read out at the top of the detector have a lower response than those at the bottom of the detector. The East stripends in the U-view planes and the West stripends in the V-view planes have the most asymmetric lengths of clear fibre cables (range $2 \rightarrow 6 \mathrm{~m}$ ). It is these stripends that have the most pronounced differences in detector response.

To assess the systematic error due to the spatial variations in detector response it is most realistic to consider the detector average over all stripends and plane views. Figure 6.19 

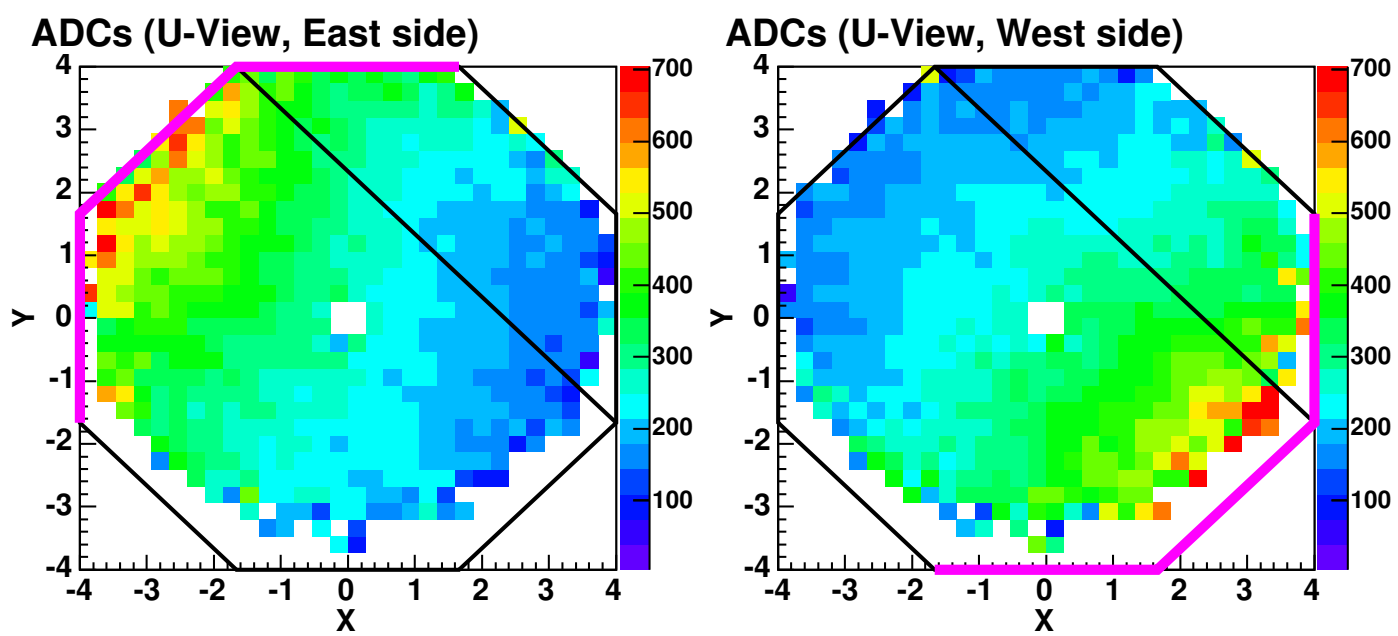

\section{SigCors (U-View, East side)}
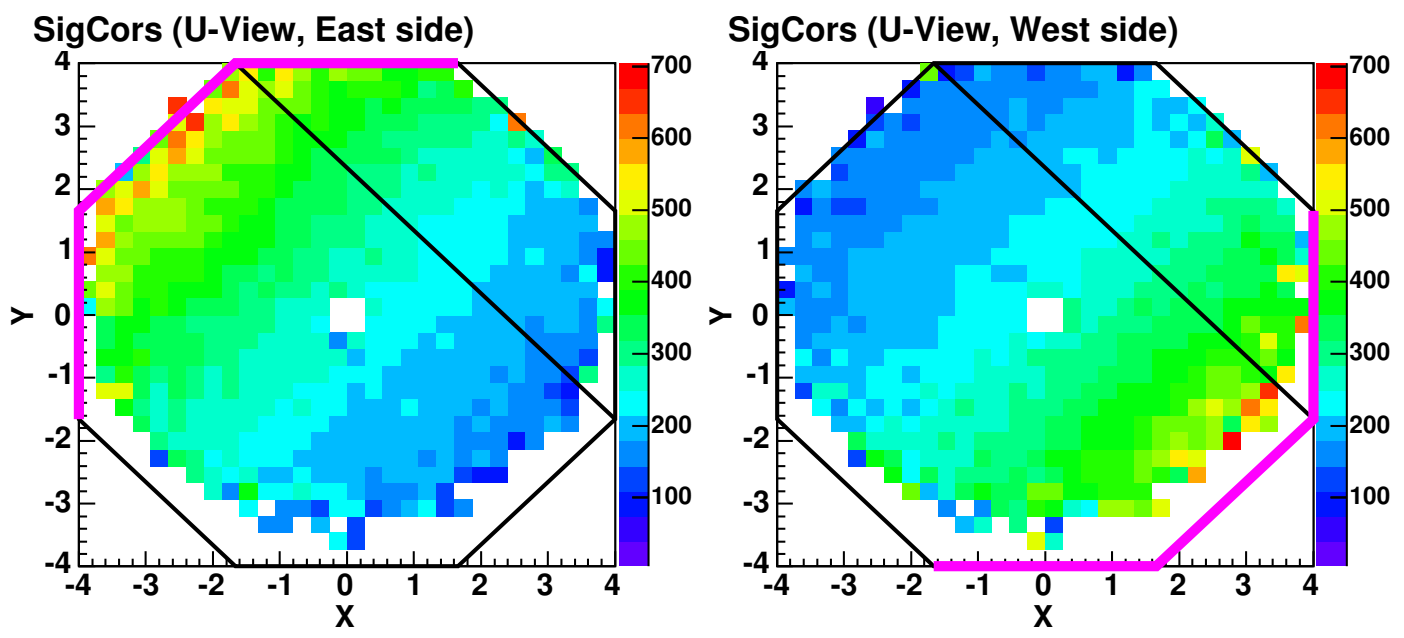

SigMaps (U-View, East side)
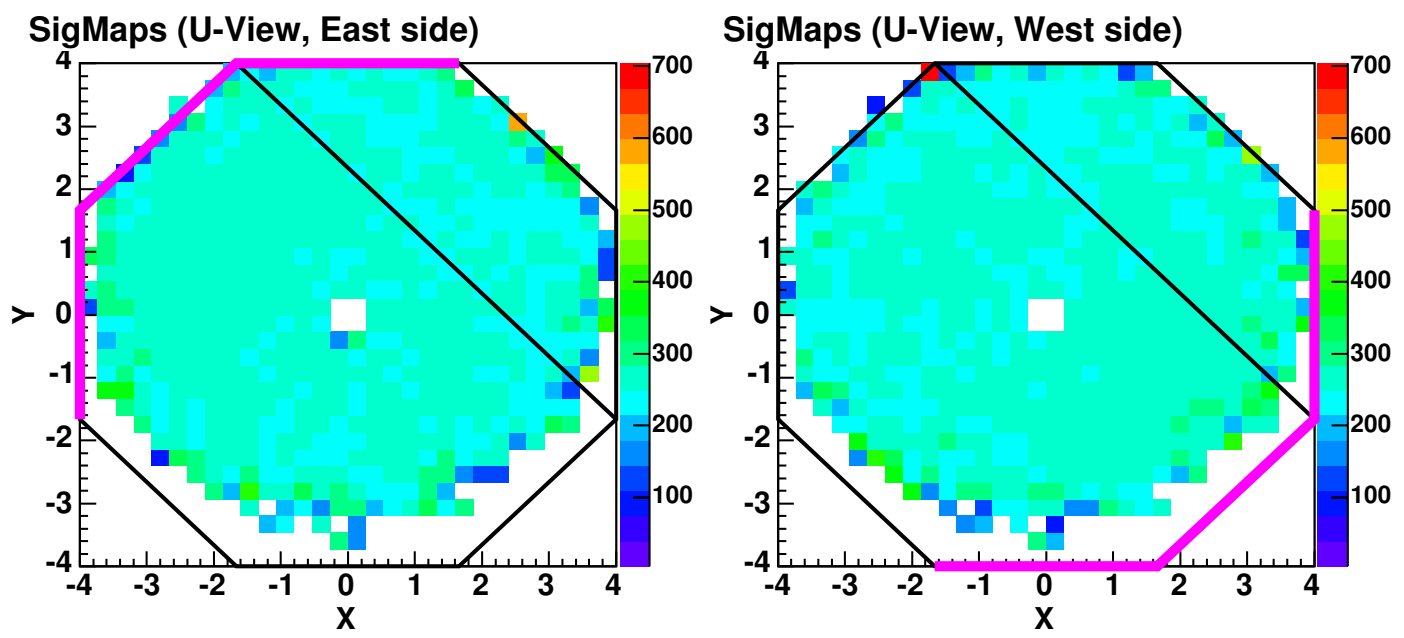

Figure 6.17: Detector response at different stages of the calibration. The response of the two stripends in U-view planes is shown for various calibration stages as a function of $x$ and $y$ coordinates. The top pair of plots show the detector response in raw ADCs for the stripends on the east and west sides of the detector. The middle pair of plots show the detector response in SigCors. The bottom pair of plots show the detector response in SigMaps. The orientation of the strips is shown by the black line across the detector face. The side of the detector read out is shown by the purple line. 

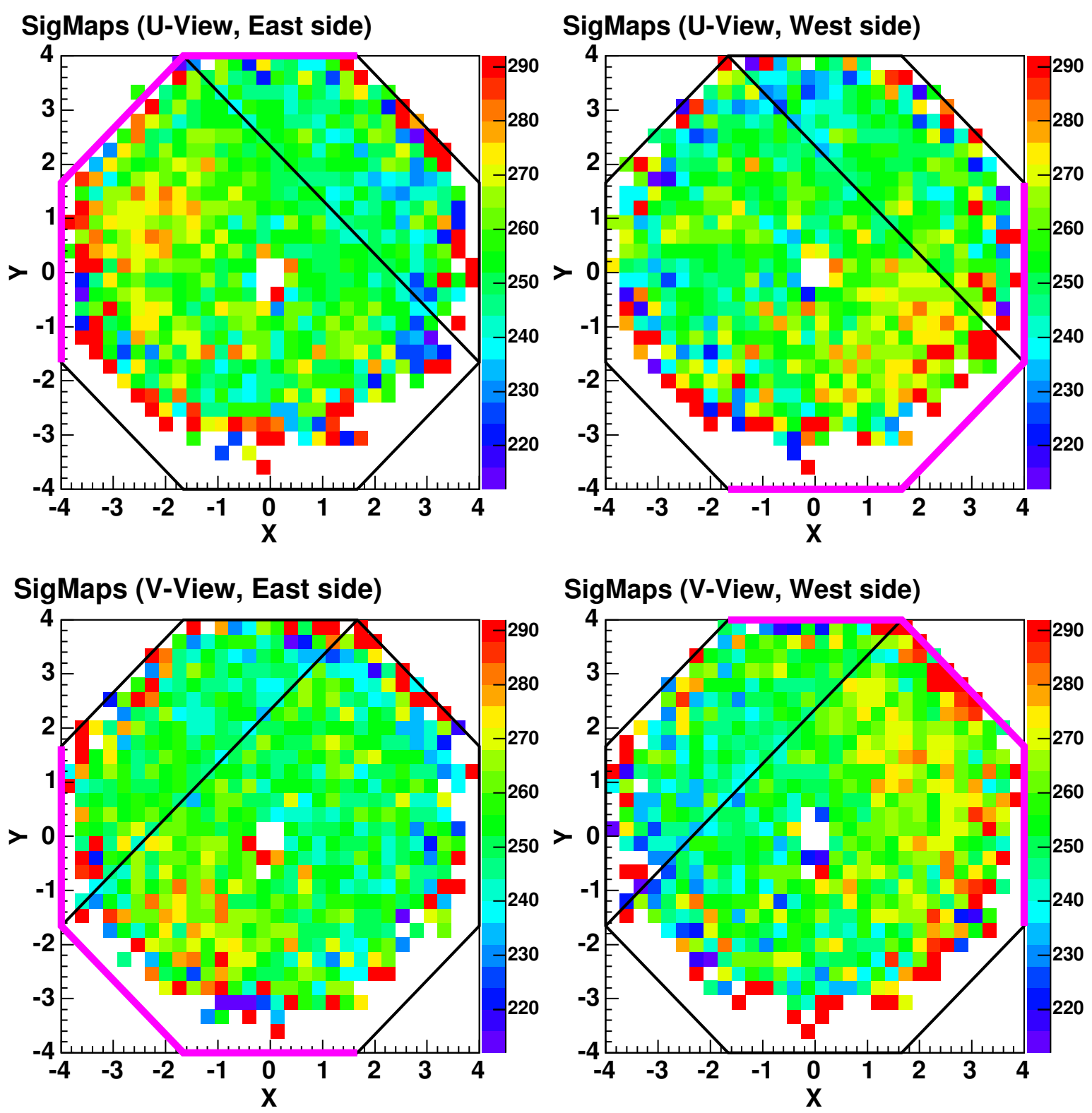

Figure 6.18: Detector response after full calibration. The top pair of plots show the fully calibrated detector response (SigMaps) for the two stripends in the V-view planes as a function of $x$ and $y$ coordinates. The bottom pair of plots show the same as the top pair but for $\mathrm{V}$-view planes. For both pairs of plots, the left (right) plot shows the detector response on the East (West) side of the detector. The orientation of the strips is shown by the black line across the detector face. The side of the detector read out is shown by the purple line. These plots are expected to show a uniform detector response in the case of perfect calibration and perfect stopping muon reconstruction. Note that the top pair of plots is just a zoom of the bottom pair of plots shown in Figure 6.17. 
shows the overall average detector response as a function of $x$ and $y$ for both data and MC in the top left and top right plots respectively. The bottom pair of plots show the 1D profiles in $x$ and $y$ separately. It can be seen that the detector response falls by $\sim 5 \%$ as a function of $y$-position in data whereas the MC is close to consistent with a flat response. The plot of detector response vs. $x$-position also shows some structure with a dip of $\sim 2 \%$ around $x=0 \mathrm{~m}$.

The reason that the differences in detector response are less when the overall detector average is considered is because the differences in response along the length of a strip cancel in part when the two stripends are summed together. Superimposing the four measurements of detector response shown in Figure 6.18 leads to the part of the detector at the top, above the coil hole having the lowest response and that is what is seen in the top left plot of Figure 6.19.

What is clear from Figure 6.19 is that the measured detector response varies significantly in data but not in MC. The effects shown in Figure 6.18 partially cancel when the two stripends are summed together and the average of the two plane views is taken. The $\sim 15 \%$ differences in detector response observed for individual stripends reduces to a $\sim 5 \%$ difference in the overall average detector response.

The fact that the differences in detector response are not observed in MC suggests that either the problem lies with erroneous calibration of the detector or that something is not modelled correctly in the MC. It is unlikely that, say, a problem with the stopping muon reconstruction could conspire with a problem in the $\mathrm{MC}$ detector simulation to give the expected response for the MC data. With the evidence presented, it is not possible to completely distinguish between the possible causes of the problems in detector response. Figure 6.18 shows that the response of the detector varies both along the length of a strip and with the strip position. It is important to point out that the regions of the detector with the highest response correlate strongly with the distance the light has to travel to the PMTs and the asymmetry of the clear fibres. It was suggested that perhaps high energy events in the rock above the detector could cause a higher response at the top of the detector 

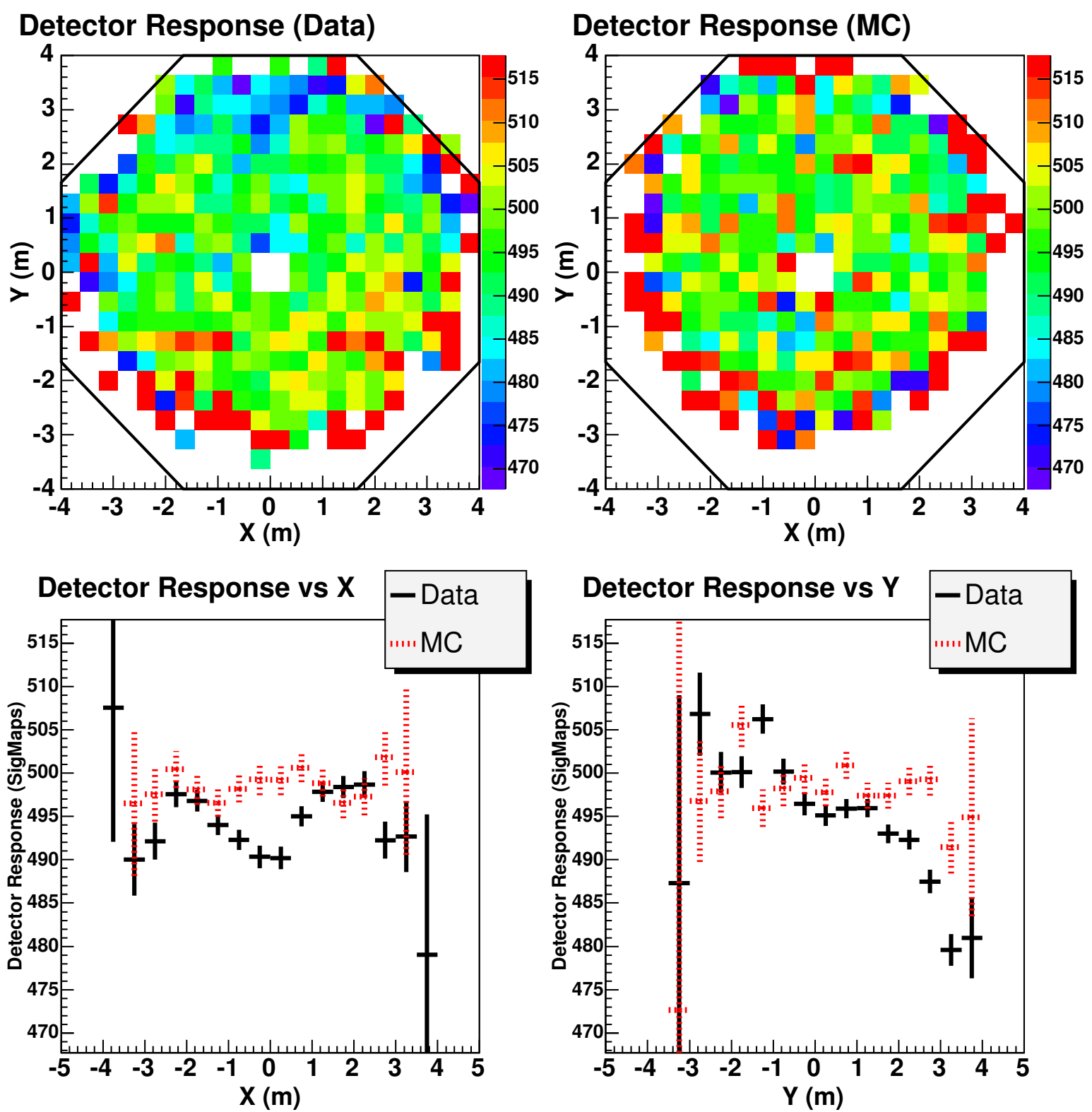

Figure 6.19: Spatial variations in the average detector response. The top pair of plots show the average detector response for all plane views and stripends in 2-dimensions as a function of $x$ and $y$. The top left plot shows the data and the top right plot shows the MC. The bottom pair of plots are $1 \mathrm{D}$ profiles that show the detector response vs. $x$ and $y$ coordinates separately. The data is shown by a solid black line and the MC by a dashed red line. The plots showing the data have a factor of two higher statistics. 
w.r.t. the bottom. However, this hypothesis does not work since regions of high response are seen towards the bottom of the detector (when those regions are closest to the PMTs). The correlations with specific geometrical features of the detector suggest that the problem is most likely to be due to the attenuation and/or strip-to-strip calibration.

In order to fully resolve the problems with the differing detector response additional work on validating the $\mathrm{MC}$ and the calibration chain is required. Many millions of through-going cosmic muon events have been collected at the Far detector, which will allow detailed studies of the attenuation and strip-to-strip calibration procedures. At the time of writing, the stripto-strip calibration procedure has not been validated with $\mathrm{MC}$ data so it is unknown if some of the problems observed are due to that calibration.

The consequence of the spatial variations in detector response is that the MEU value changes depending on where a neutrino event happens to occur in the detector. The neutrino events can occur anywhere in the Far detector and they are likely to produce showers that span several planes. Thus, it makes sense to consider the variations in the overall average detector response (see Figure 6.19) rather than the variations in response of a single stripend in a single plane view (see Figure 6.18). As was described above for the temporal variations in detector response, the effect on the neutrino measurements of the variations in detector response depend on the details of such a measurement. The maximum spatial variation in overall detector response is $\sim 5 \%$, thus the error on the calorimetric energy measurement of a single neutrino is set to $\pm 2.5 \%$. However, in the case of the atmospheric neutrino analysis with, say, 150 neutrino events in the 15 month period considered then the error on the MEU value is only $\sqrt{150 \times 2.5^{2}} / 150=0.2 \%$. For beam neutrinos numbering in the (low) thousands the error will be even less.

In quoting an error of $0.2 \%$ it is assumed that the MEU value is calculated with a uniform distribution of stopping muons. However, this is not the case as the stopping cosmic muons are all downward going. Figure 6.20 shows the distribution of strips included in the track window as a function of $\mathrm{x}$ and $\mathrm{y}$ position. It can be seen that the vast majority of stopping muons are concentrated in the top half of the detector. The definition of a MEU means 


\section{Spatial Distribution of Strips in Track Window}

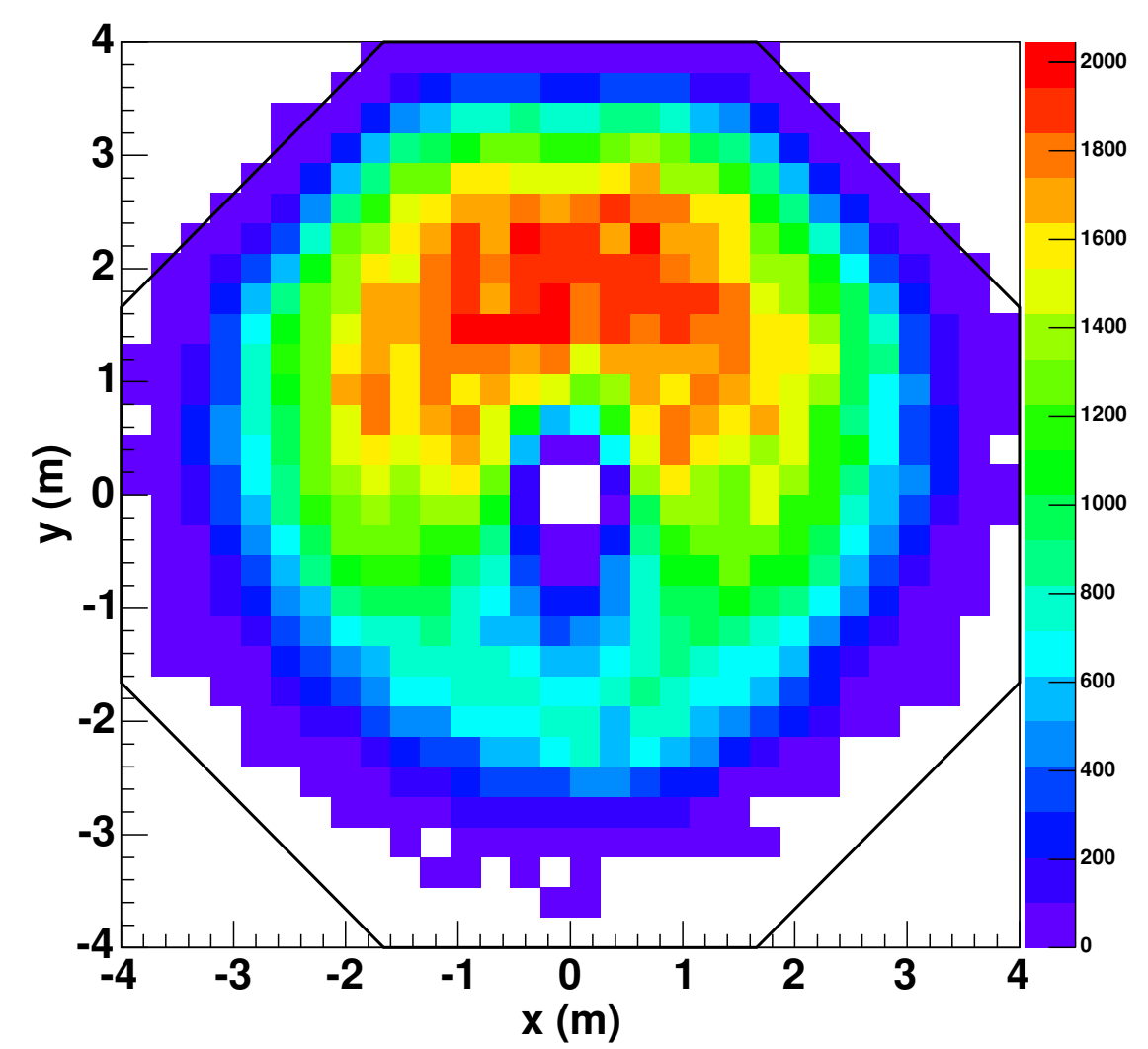

Figure 6.20: Spatial distribution of strips in track window.

that the measured detector response is weighted by the greater number of events in the top of the detector. To calculate the required correction factor to the MEU value, due to the imbalance of the location of the stopping muons in the detector, a calculation is performed. A 2-dimensional profile histogram is made using the average $\mathrm{x}$ and $\mathrm{y}$ position of the strips in the track window and the sum of the signal in the track window for each muon. A loop over all the bins in the 2D histogram is made and the average signal in the track window for each bin is obtained. The average of all the bins that have their centre within a rough fiducial volume (defined as within $3 \mathrm{~m}$ from the detector centre) is calculated. It is found that this uniformly-weighted average detector response is only $0.6 \%$ greater than the average of all the stopping muons.

A correction for the spatial variations in the detector response of $+0.6 \%$ is made to the measured MEU value. The size of the error on this correction is not well understood but it is conservatively estimated to be $100 \%$ of the size of the correction. Thus, the total error on the 
MEU value due to the spatial variations in response is estimated to be $\sqrt{0.2^{2}+0.6^{2}}= \pm 0.6 \%$. Although, as a word of caution, it should be pointed out that the error due to the spatial variations could be as large as $\pm 2.5 \%$ depending on the number of neutrino events used and their spatial distribution in the detector. However, the lower value of $\pm 0.6 \%$ is most likely to apply to the neutrino analyses that MINOS will perform.

The discovery of the spatial variations has proved the track window technique to be a valuable tool for observing problems with the detectors. The high spatial resolution and the near uniform energy deposition makes the track window technique a sensitive probe of detector response. Although the error from the spatial variations is not likely to cause a significant error on the measurements MINOS makes it is unsatisfactory from a number of view points: the detector is not yet fully understood and there could be a deeper problem; the MC simulation does not currently reproduce the detector response accurately (due to perfect calibration in MC, see section 6.4.1); the calorimetric energy resolution is degraded; and the neutrino oscillation results are potentially susceptible to biases. A programme of work is currently underway to try and understand the spatial variations shown in this thesis.

\section{Backgrounds}

As discussed in section 6.2.2, the main background to the cosmic stopping muon sample in the Far detector is actually other cosmic muons. However, the background from high energy cosmic muons is successfully reduced to a tiny fraction of the event sample. In MC only $1 / 1000$ of the events used in the relative calibration are actually high energy through-going cosmic muons. The systematic error from this small background is negligible.

A background that is observed at the Far detector is from high energy muons that undergo a catastrophic energy loss and then stop in the fiducial volume. High energy muons can lose a significant fraction of their energy by emitting Bremsstrahlung. The resulting electromagnetic shower from the Bremsstrahlung can enter the track window and give an artificially high response. Figure 6.21 shows a high energy muon in the Far detector that loses all its energy in a huge shower before stopping. This event is included in the stopping 


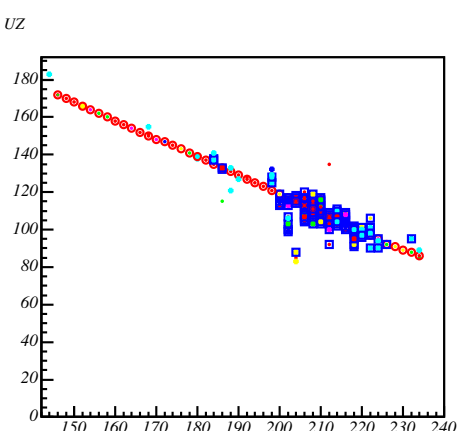

Plane Charge PE

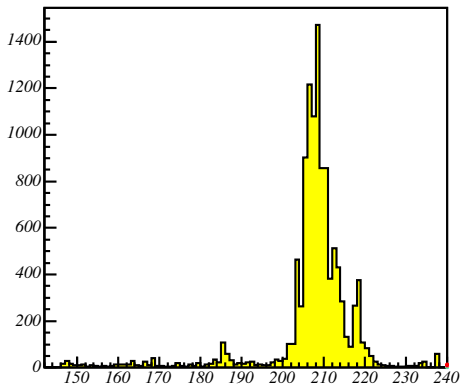

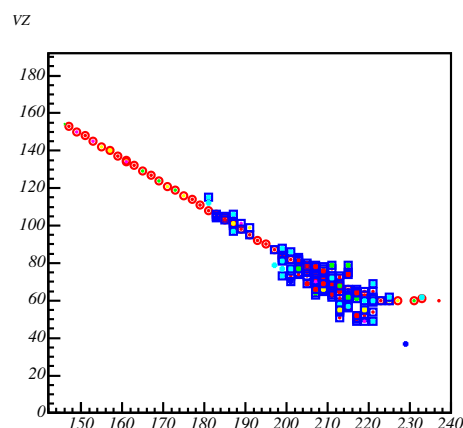

Run : 20986 Snarl: 65014 Trk: 1 Shw: 2
$X Y$
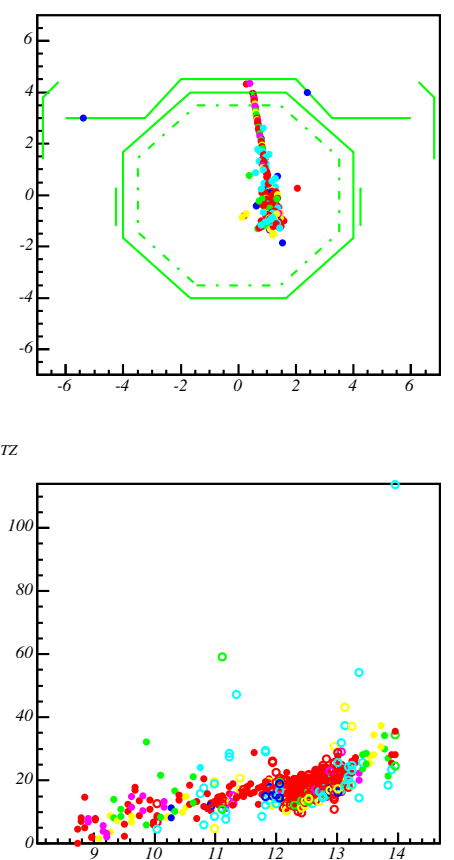

Figure 6.21: Catastrophic muon energy loss. This event display shows a high energy muon entering the Far detector and undergoing a catastrophic energy loss before stopping inside the fiducial volume. A huge shower can be seen with the muon just emerging on the RHS. The UZ and VZ plots show the position of the hits in strip and plane co-ordinates for the two views. The tracked hits are shown with red circles and the hits in the shower are shown with blue squares. The $x y$ plot shows the end-on view (looking South-East). The bottom left plot shows the charge deposited in pe vs plane number. The bottom right plot shows the time of the hits as a function of z-coordinate.

muon sample since it passes all the cuts. The detector response in the track window for this event is a factor of 20 more than the average. Such events are rare but their energy deposition is sufficiently large that they are potentially significant. The obvious solution would be to remove events with huge energy depositions but the difficulty is in deciding on the threshold. Figure 6.22 shows how the mean and median of the detector response in the track window change as the upper threshold is varied. It can be seen that the mean detector response changes by $1 \%$ as the truncation threshold is lowered from $20000 \rightarrow 1000$ SigMaps. However, because a MEU is defined as the median track window response (see equation 5.8) these rare events have a negligible impact on the MEU value. 


\section{Detector Response in Track Window}
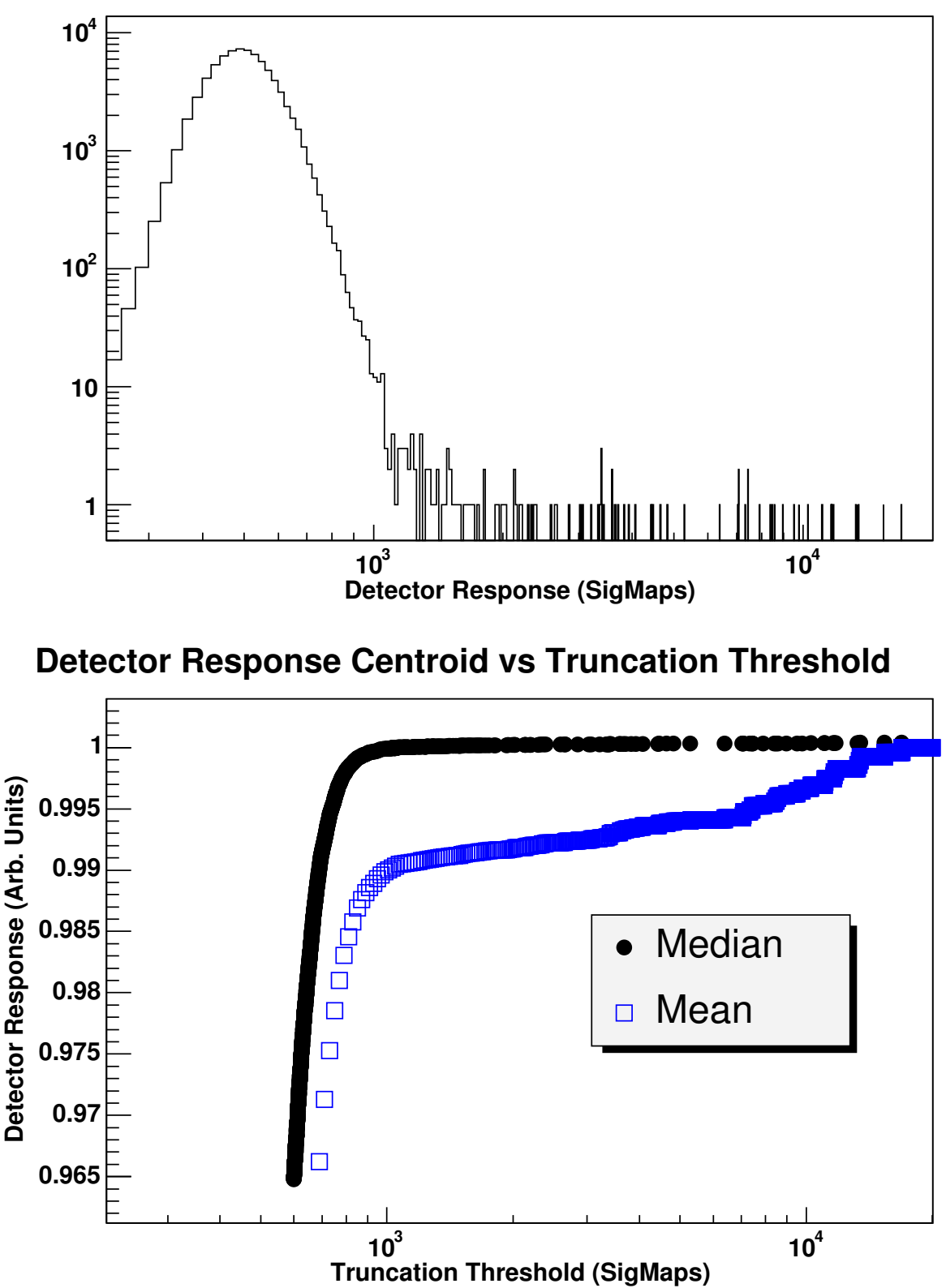

Figure 6.22: Detector response centroid vs. truncation threshold. The top plots shows the distribution of detector response in the track window. The bottom plot shows how the centroid (mean and median) of the detector response distribution changes as an upper truncation threshold is applied. The two plots have been seperately normalised such that the response with no truncation threshold is the same. 


\section{Angular Dependence}

One significant difference between the sample of muons used at the Calibration detector and the Far detector is the angular spectrum. The beam muons used at the Calibration detector all travel perpendicularly to the planes while at the Far detector the muons enter the detector at many different angles and are on average much steeper. The top plot in Figure 6.23 shows the detector response as measured in the track window as a function of angle after the correction for path length through the scintillator has been applied. The bottom plot shows the angular distribution of the stopping muons as measured in the track window. From the top plot it can be seen that for data the detector response increases by approximately $1 \%$ between $\left|\operatorname{Cos}\left(\theta_{z}\right)\right|=1 \rightarrow 0.3$.

The muons with $\left|\operatorname{Cos}\left(\theta_{z}\right)\right|<0.3$ are not used for the analysis presented in this thesis. Since the average $\left|\operatorname{Cos}\left(\theta_{z}\right)\right|$ in the sample of muons used is approximately 0.6 , the measured detector response is estimated to be $0.5 \%$ greater than the value that would be obtained if just horizontal muons were used.

There are two main reasons for this angular dependence. Firstly, the shape of the energy deposition in the scintillator changes with the path length through the scintillator due to the effects of stochastic energy losses. Essentially, the thicker the scintillator the greater it's ability to absorb all the energy from an energetic delta-ray, which causes the high energy tail, or "Landau tail", of the energy deposition distribution to be extended (see Figure 5.5 and the top plot of Figure 6.22). However, taking the median of the energy deposition distribution limits the sensitivity to this Landau tail (as was the case with the catastrophic energy losses described above in this section). Secondly, the average momentum of a muon in the last plane of scintillator it crosses depends on the steepness of the track. Muons only occasionally come to a complete stop in the scintillator, it is much more likely that they will lose the last of their kinetic energy in passive detector elements beyond the end of their track. The end of the track, however, is determined by the last scintillator plane to be hit. Figure 6.24 shows the energy of the stopping muons at the end of their track in a MC simulation of the Far and Calibration detectors. The average energy of a muon at 


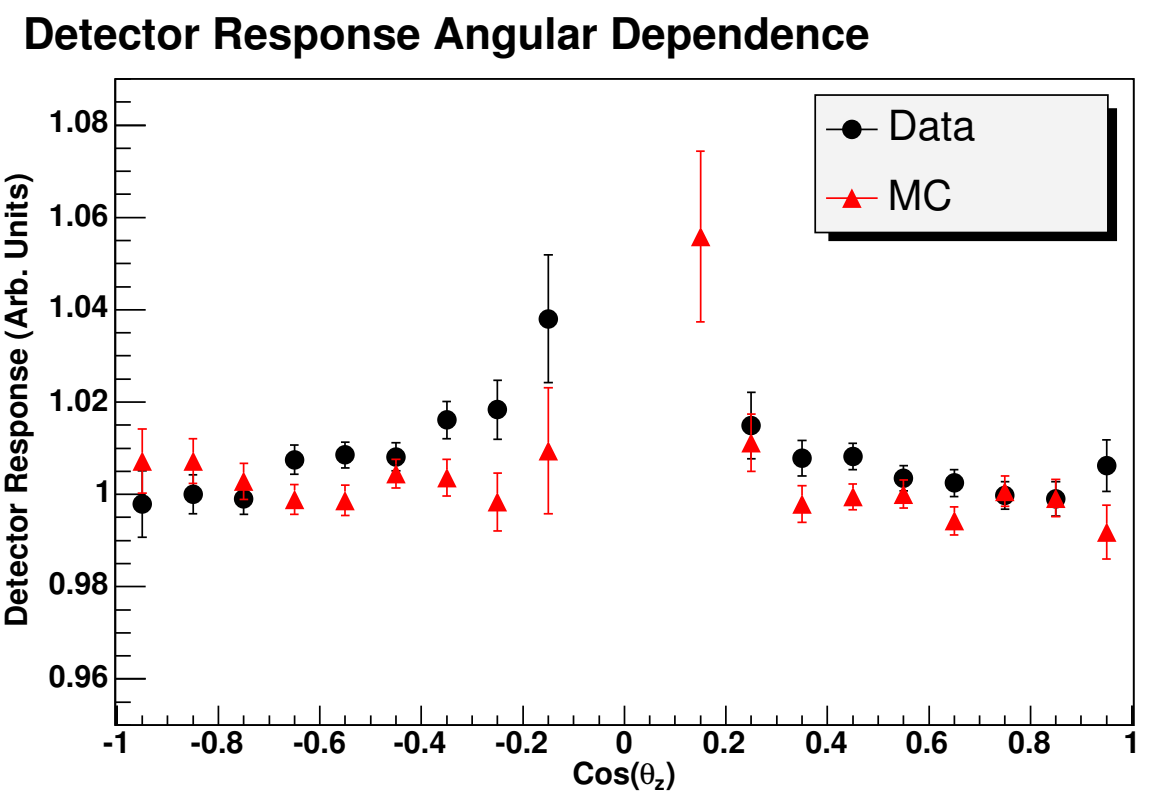

\section{Angular Distribution of Stopping Muons}

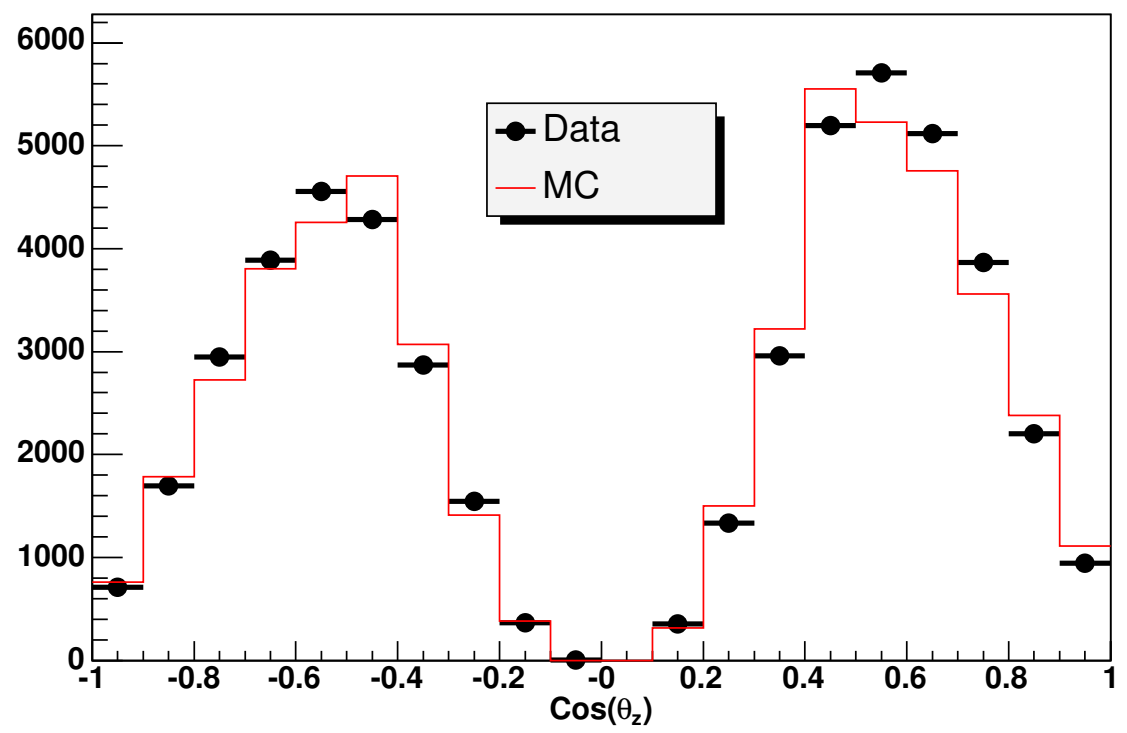

Figure 6.23: Detector response angular dependence and the angular distribution of stopping muons. The top plot shows the detector response vs. the angle of the muon in the track window for both data and MC. The bottom plot shows the angular distribution of the stopping muons as measured in the track window. The data and MC both have the magnetic field in the "forward" direction (such that the field focuses $\mu^{-}$created by beam neutrinos). 
the end of its track in the Far detector is $0.19 \mathrm{GeV}$, whereas in the Calibration detector the average energy is only $0.15 \mathrm{GeV}$. The main reason for this difference is that the muons in the Calibration detector all enter the detector perpendicular to the planes. The steep muons in the Far detector lose more energy in the last plane of steel they enter and thus have a higher energy in last plane of scintillator they cross. The consequence of this higher energy at the track end point is that the track window is placed further up the relativistic rise than it should be. The $\mathrm{dE} / \mathrm{dx}$ of a muon on the relativistic rise only changes by approximately $1 \%$ for every $100 \mathrm{MeV}$ increase in muon energy for momenta between $0.5 \rightarrow 1.5 \mathrm{GeV}$. Thus, a $40 \mathrm{MeV}$ difference in the average stopping muon energy at the end of the track causes a systematic shift in the track window energy deposition of $\sim 0.4 \%$. In determining the $40 \mathrm{MeV}$ shift in energy all angles were considered, thus the $0.4 \%$ is a slight overestimate as only tracks with $\left|\operatorname{Cos}\left(\theta_{z}\right)\right|>0.3$ are used. It can be seen that the estimate of the increase in the measured calorimetric energy response due the change in energy at the track end is less than that measured using the angular distribution above. This suggests that the change in the shape of the energy deposition distribution with angle, due to the Landau tails, is also contributing to the measured angular dependence.

A correction of $-0.5 \%$ is made to the MEU value due to the measured angular dependence of the detector response and the sample of muons used. The size of the error on this correction is not well known but it is conservatively estimated to be $100 \%$ of the size of the correction. Thus, the error due to the angular dependence, after the $-0.5 \%$ correction to the MEU number, is $\pm 0.5 \%$.

\section{Detector Performance}

The performance of the detector can affect the data quality and change the measured value of a MEU. There are three main ways in which detector performance can have an effect on the relative energy calibration. Firstly, any dead electronics chips will reduce the detector response in the track window. Secondly, if chips are malfunctioning and registering a false signal then the response in the track window will be artificially high. Thirdly, dead chips 


\section{Muon Energy at Track End}

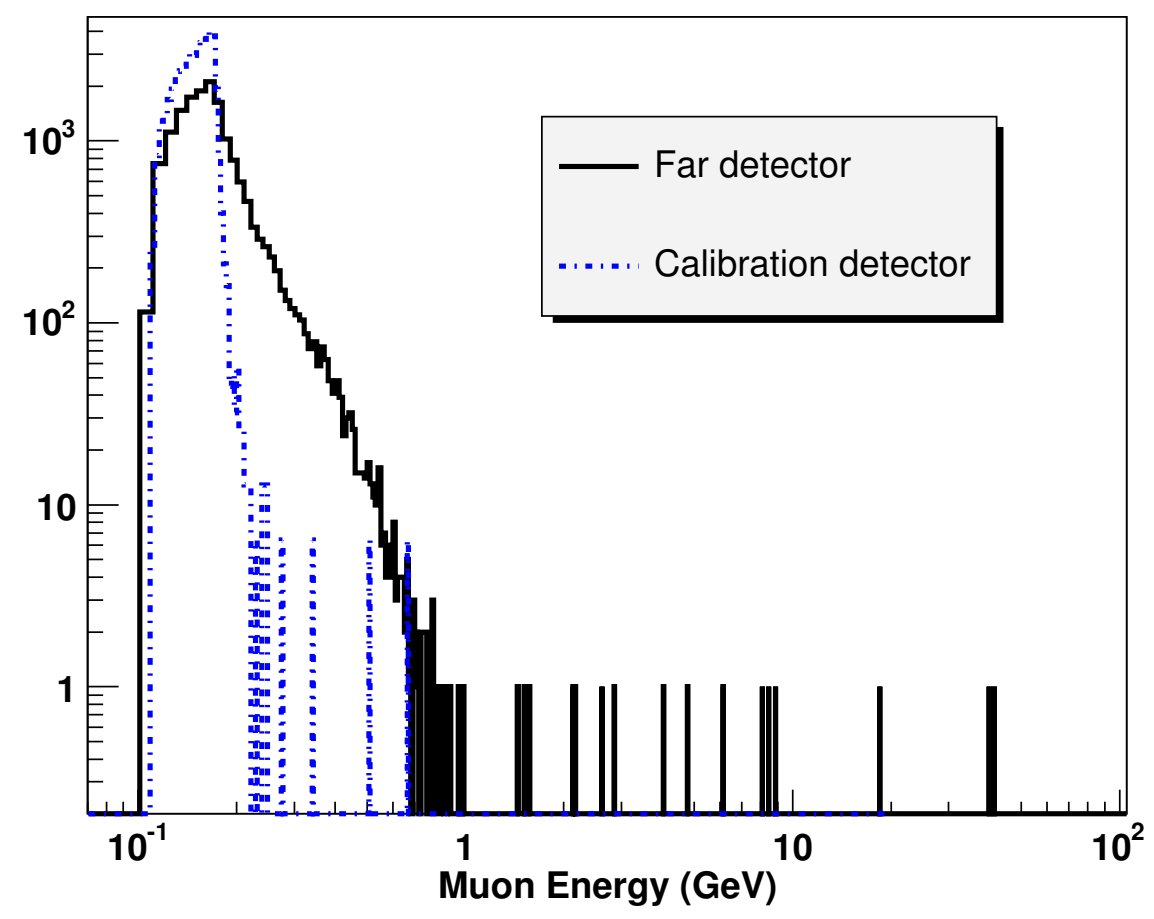

Figure 6.24: Muon energy at the track end. A MC simulation was used to determine the lowest energy of the muons as they traversed the Far detector and the Calibration detector. The distribution of energies for muons that pass all the selection cuts is shown. The histogram for the Calibration detector has been normalised to the Far detector histogram so that the effective number of events is the same. 
near the muon track end-point have the potential to make a through-going cosmic muon appear partially contained. An analysis of the effect of dead chips has been performed and is presented here. Although events with dead chips in the track window are cut out it is informative to check that such a cut gives the expected result since not all dead chips can be detected.

Over the period during which the data used for this thesis was taken the performance of the Far detector improved significantly. At the start of the period approximately $0.34 \%$ of chips $^{2}$ were busy in each stopping muon event. By the end of the period the number of busy chips had fallen to approximately $0.21 \%$.

One potential systematic error that can arise with determining the rate of busy chips is that only chips with hits above the sparsification threshold are used. It is possible for a chip to be busy digitising a hit that then never appears in the data stream because it was sparsified away in the front-end electronics. The LI system provides a way of determining the rate of these "invisible" busy chips, which have hits below the sparsification threshold. It is known how many flashes the LI system delivers and this can be compared with the number flashes seen by each chip. An analysis of LI data taken near the end of the data period used in this thesis revealed that the total rate of busy chips was $0.24 \%$. Comparing this LI value with the rate from busy chips that aren't sparsified suggests that the rate of "invisible" busy chips is only $0.03 \%$, which is negligible. A measurement of the effect of dead chips on the detector response in the track window yields a value of $0.2 \%$ as expected.

A further systematic error that can arise from busy or cold chips is that through-going cosmic muons can appear partially contained. The presence of dead chips just beyond the apparent end of the track and their effect on the MEU number has to be considered. Table 6.6 lists the observed and expected probabilities of one or more chips to be busy or cold in the planes beyond the apparent end of the track. The expected probabilities of chips being dead are calculated by considering what the probability of them all being live is, and then subtracting that value from 1 . The observed and expected rates agree well. The amount

\footnotetext{
${ }^{2}$ Chips reading out the veto shield are excluded from this analysis.
} 


\section{Number of planes beyond track end} 5 3 2 1
Observed fraction of events with dead chips 0.033 0.022 0.016 0.007
Expected fraction

of events with dead chips

0.030

0.018

0.012

0.006

Table 6.6: Rate of dead chips in planes just beyond track end point at the Far detector. The observed and expected rates of dead (busy or cold) chips in the planes just beyond the apparent end of the track are listed.

of contamination from through-going muons is in the worst case $<0.3 \%$. Thus, the effect of this potential contamination on the MEU value is determined to be negligible.

\section{Gaps Within Scintillator Planes}

Within each detector plane there are gaps in the active scintillator, which lowers the light output of a scintillator plane. This can be thought of effectively lowering the average amount of active material traversed by a muon in 1 plane of scintillator.

The effect of the gaps on the relative calibration is zero by definition, as explained in section 4.5.5 (to put it simplistically, an MEU is the response per plane however that plane is constructed). However, it is worth noting that the quantity of energy in GeV associated with $1 \mathrm{MEU}$ of detector response is dependent on the size of the gaps.

\subsubsection{Summary of Errors and Conclusions}

The statistical error on the measurement of the relative calorimetric energy response of the Far detector is a negligible 0.1\%. A summary of the systematic errors (described in section 6.2.4) on the Far detector MEU value is given in Table 6.7. Adding the systematic errors in quadrature gives a total systematic error of $0.9 \%$ on the measurement of the Far detector's relative calorimetric response.

Consider the potential sources of correlation in the systematic errors. The error due the spatial variations and the angular dependence arise due to different (and independent) 


\section{Error description}

1.) Temporal drifts in detector response

2.) Spatial variations in detector response

3.) Angular dependence
Percentage error

$\pm 0.2$

$\pm 0.6$

$\pm 0.5$

Table 6.7: Systematic errors on the Far detector MEU value.

mechanisms. The angular dependence arises from the changing muon momentum at its measured end-point and the changing shape of the energy deposition distribution. Whereas, the spatial variation in response correlates with the distance the light travels to the PMTs. No realistic mechanisms are known that could cause the muons to be distributed such that their angular dependence could mimic the dependence on distance to the PMTs. It has been shown that the temporal fluctuations correlate strongly with the drift in the temperature of the Far detector cavern, as expected from previous measurements. There is no known realistic mechanism that could explain a temperature dependence of the spatial variations or the angular dependence.

It should be noted that, as is stated in section 6.2.4, the estimate of the systematic errors arising from the temporal and spatial variations in detector response is evaluated in the context of a neutrino measurement. It is assumed that the neutrino events will be distributed uniformly in time and across the fiducial volume of the detector such that the variations in response average out. This is considered a good assumption given that the numbers of events will be in the thousands for the beam neutrinos and in the hundreds for the atmospheric neutrino analysis. In any event, it is expected that the LI drift calibration and through-going muon drift calibration will soon reduce the temporal fluctuations to a negligible level. Determining the cause of the spatial variations in detector response may take more time but a correction for the observed effects can be made.

The third error in the table is more intrinsic to the track window technique, although there is still room for improvement on what is presented in this thesis. The error due to the angular dependence could be avoided by only using muons that travel perpendicularly to the planes, although a balance with the statistical precision would need to be met.

In conclusion, a precise measurment of the relative calorimetric response of the Far de- 
tector has been accomplished using the track window technique. The total error associated with this measurement is only $0.9 \%$.

\subsection{Measuring the Bethe-Bloch Curve}

The stopping power for $\mu^{+}$in copper $^{3}$ as a function of muon momentum is shown in Figure 5.1. For stopping muons of the momenta of interest to MINOS $(0.1 \rightarrow 5.0 \mathrm{GeV} / \mathrm{c})$ the form of the stopping power curve is given by the Bethe-Bloch equation [23]. Using this equation it is straight forward to calculate the stopping power for a range of materials, including the scintillator used in MINOS. The variation of stopping power with muon momenta is referred to as the "Bethe-Bloch curve" in this thesis.

Measurements of the Bethe-Bloch curve are made in order to cross-check that the reconstruction and calibration of stopping muons at the different detectors is working correctly. The Bethe-Bloch curve measured in data is compared with that measured in the full GEANT MC. In addition, the measurements in data and MC are compared with the calculated Bethe-Bloch curve.

\subsubsection{Bethe-Bloch Measurements at the Far Detector}

To measure the Bethe-Bloch curve the same sample of stopping muons obtained for the relative calibration is used. The measured detector response is converted to $\mathrm{MeV} \mathrm{cm} / \mathrm{g}$ such that the minimum ionising response of the data and full GEANT MC agree with the Bethe-Bloch calculation. The momentum of the muon is determined by using the rangeenergy relation that has been calculated for muons in the MINOS detectors. Figure 6.25 shows the stopping power for muons in scintillator as a function of muon momentum. The stopping power calculated using the Bethe-Bloch equation is shown with the green stars, the measurement of the stopping power in data and MC is shown with the black circles and red

\footnotetext{
${ }^{3}$ The stopping power for muons in copper is only $3 \%$ different from that in iron in the region of interest as explained in section 5.1.1.
} 


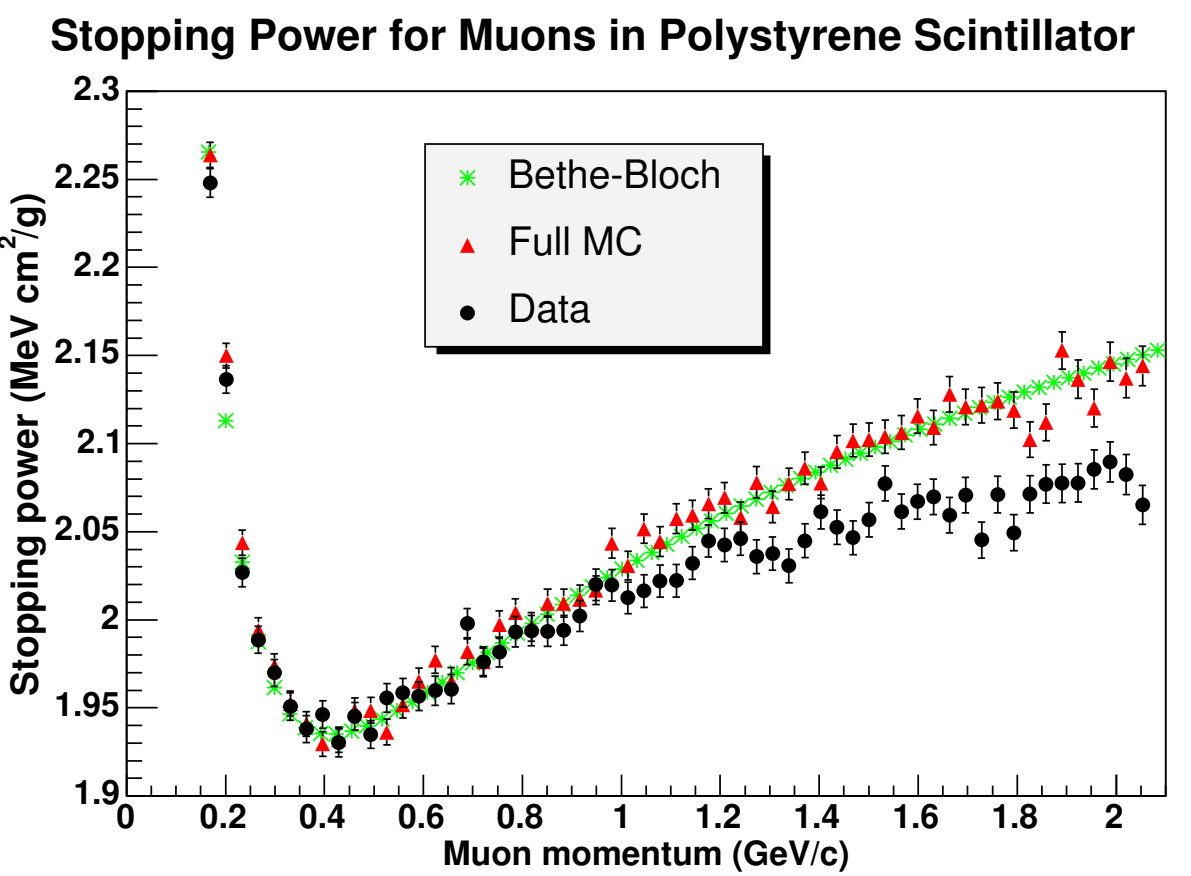

Figure 6.25: Bethe-Bloch curve for the Far detector. The stopping power calculated using the Bethe-Bloch equation is shown with the green stars, the measurement of the stopping power in data and $\mathrm{MC}$ is shown with the black circles and red triangles respectively.

triangles respectively. It can be seen that the Bethe-Bloch calculation and the full GEANT MC agree very well. Whereas, the data shows a significant deviation from the expected stopping power above $1 \mathrm{GeV} / \mathrm{c}$.

It is the spatial variations in detector response (described in section 6.2.4) that are the cause of the drop in stopping power at higher muon momenta. The stopping muons are all coming from above the detector. Thus, on average the momenta of the stopping muons is higher at the top of the detector. Figure 6.19 shows that the fully calibrated detector response is $\sim 5 \%$ higher at the top of the detector than at the bottom. This difference in detector response causes the stopping muons to have an artificially low response when they enter the top of the detector, which is also when they have higher average momenta.

To show that the spatial variations in detector response were the cause of the drop in stopping power at higher muon momenta a correction to the observed $y$-dependence of the detector response is made. A simple parametrisation of the $y$-dependence was constructed by using two straight line fits. The slope of the detector response vs. $y$-coordinate (shown in the bottom right plot of Figure 6.19) can be seen to increase rapidly between $y=1 \rightarrow 4 \mathrm{~m}$. 


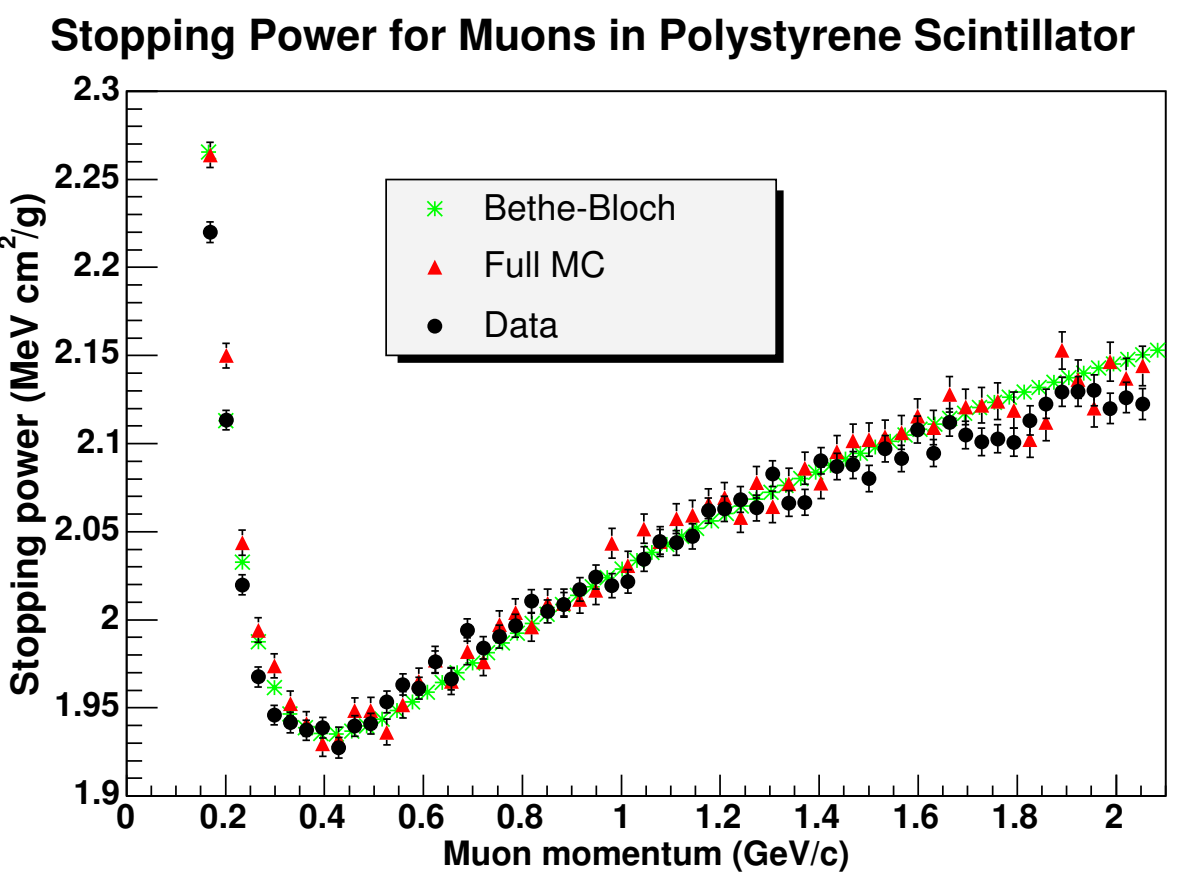

Figure 6.26: Bethe-Bloch curve for the Far detector after correction. The data curve shown in Figure 6.25 has been corrected for the $y$-dependence of the fully calibrated detector response. The stopping power calculated using the Bethe-Bloch equation is shown with the green stars, the measurement of the stopping power in data and $\mathrm{MC}$ is shown with the black circles and red triangles respectively.

Thus, the first straight line fit is obtained between $y=-4 \rightarrow 1 \mathrm{~m}$ and the second straight line fit is obtained between $y=1 \rightarrow 4 \mathrm{~m}$. Exactly where the correction is made relative to is not of consequence since it only affects the normalisation, which is fixed to the muons' minimum ionising response. Figure 6.26 shows the Bethe-Bloch curve after the correction for the spatial variations in detector response has been made. It can be seen that the stopping power for momenta above $1 \mathrm{GeV} / \mathrm{c}$ now agrees reasonably well with the $\mathrm{MC}$ and the BetheBloch calculation. It should also be pointed out that the agreement between the 3 curves has got slightly worse on the left hand side of the minimum. However, given the simple nature of the correction this is not surprising. The aim of the exercise was to demonstrate the cause of the problem shown in Figure 6.25. The long term solution is to understand the cause of the spatial variations in the detector response and fix the problems. 


\subsubsection{Bethe-Bloch Measurements at the Calibration Detector}

As with the Far detector, the sample of stopping muons used in the relative calibration is also used to measure the Bethe-Bloch curve. The same procedure, as described above for the Far detector, is used to determine the stopping power and the muon momentum. In addition, there are a number of details specific to the Calibration detector. Firstly, a sample of $1.8 \mathrm{GeV} / \mathrm{c}$ muons from the T11 2002 run period are used. Secondly, plane zero is not used in the Bethe-Bloch measurement due to its poor calibration, which means the highest momentum point is missing in the plots presented below.

Figure 6.27 shows the stopping power for muons in scintillator as a function of muon momentum for the Calibration detector. The stopping power calculated using the BetheBloch equation is shown with the green stars, the measurement of the stopping power in data and MC is shown with the black circles and red triangles respectively. It can be seen that both the data and the full GEANT MC do not agree with the Bethe-Bloch calculation at high momenta $(>\sim 1.5 \mathrm{GeV} / \mathrm{c})$. In addition, the data shows deviations from both the Bethe-Bloch and the MC above $\sim 1 \mathrm{GeV} / \mathrm{c}$.

Consider first the fall in stopping power above $\sim 1.5 \mathrm{GeV} / \mathrm{c}$. In this region the stopping power measured in data and MC does not match that predicted by the Bethe-Bloch equation. In contrast, the same plot for the Far detector (Figure 6.26) does not show this feature. A study of the true energy deposition in the MC reveals that in the first few planes of the Calibration detector (i.e. those planes where the muons have high momenta) the true energy deposition is lower than expected, which indicates that the effect is not caused by problems in the reconstruction or calibration. The hypothesis for the cause of this effect is related to the fact that showers of delta rays are believed to develop progressively along the muon trajectory [105]. Delta rays that can penetrate a few planes are produced by the muons as they traverse the detector. Thus, a plane deep in the detector records energy depositions from delta rays produced in the previous planes. Whereas, the planes close to the front of the detector will not see as many delta rays, since virtually none are produced in the air at the front of the detector. The reason that this effect is not observed at the Far detector 


\section{Stopping Power for Muons in Polystyrene Scintillator}

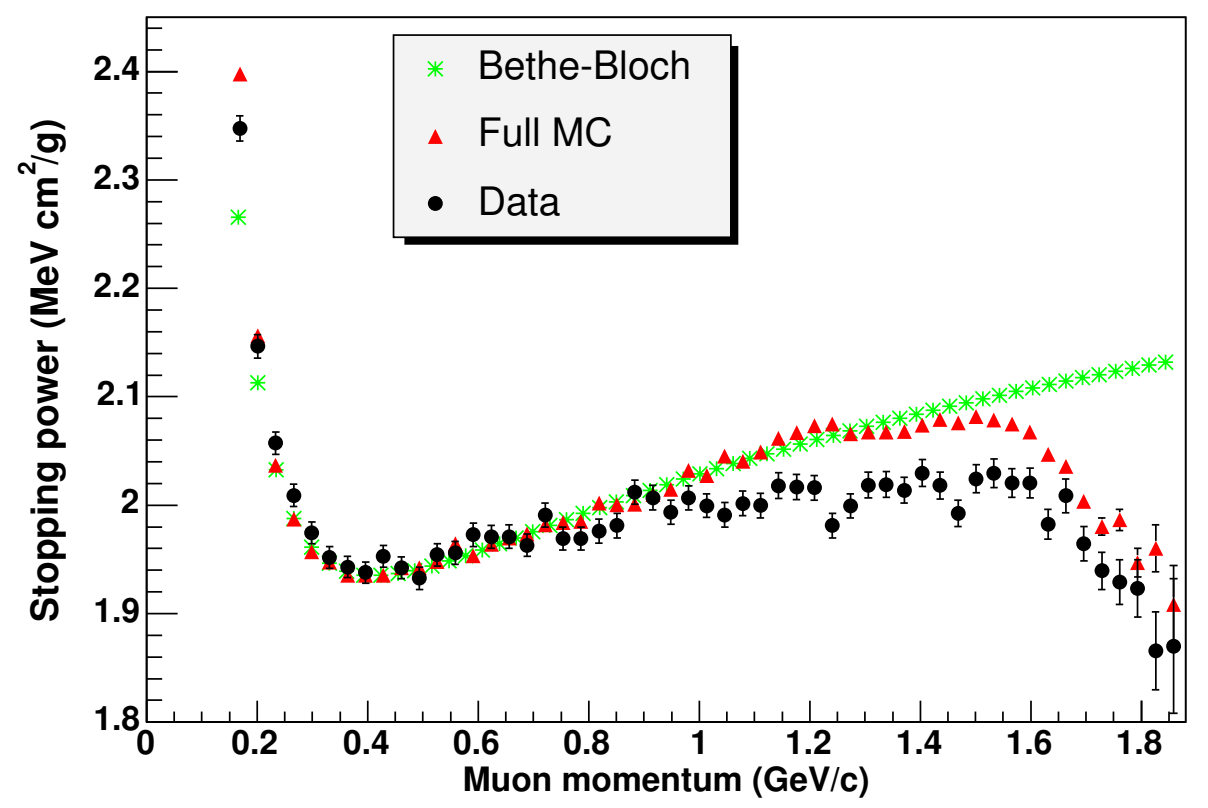

Figure 6.27: Bethe-Bloch curve for the Calibration detector. The stopping power calculated using the Bethe-Bloch equation is shown with the green stars, the measurement of the stopping power in data and MC is shown with the black circles and red triangles respectively.

is because the tight containment cuts require that the muons be deep in the detector when they stop. The systematic effect of this shower-development feature on the MEU number is negligible since the track window samples the muon between $0.5 \rightarrow 1.1 \mathrm{GeV} / \mathrm{c}$. The lowest momentum muons used are $1.4 \mathrm{GeV} / \mathrm{c}$.

Consider now the disagreement of the data with both the Bethe-Bloch and the MC above $\sim 1 \mathrm{GeV} / \mathrm{c}$ (as shown in Figure 6.27). To understand this effect it is necessary to consider the spectrum of muons in the Calibration detector and the spread in the range of muons of a given energy. Figure 6.28 shows the spread in the muon track length and its effect on the measured stopping power. The top plot shows the distribution of muon track lengths. Data is shown with the black points and $\mathrm{MC}$ with the solid red line. The peak in the distribution is split into three separate ranges, labelled A, B and C. The middle plot shows the stopping power for muons in polystyrene scintillator from a Bethe-Bloch calculation and as measured in $\mathrm{MC}$ for the three separate ranges. The blue circles, turquoise triangles and purple squares show the stopping power for ranges $\mathrm{A}, \mathrm{B}$ and $\mathrm{C}$ respectively. The green stars show the stopping power obtained from a Bethe-Bloch calculation. The bottom plot shows the same 
as the middle plot except data instead of MC is displayed.

In the top plot of Figure 6.28 it can be seen that the track length distribution for muons in data is quite different from that in MC. The track length distribution for data has a significant contribution from the lower "off-momentum" muons that stop short of the peak at $\sim 3 \mathrm{~m}$ (see section 6.1.1). In the middle plot it can be seen that the Bethe-Bloch curves for the three ranges of track length (A, B and C) are significantly different. The BetheBloch curve for the muons with short track lengths (range A) rises significantly faster with increasing momentum. Conversely, the Bethe-Bloch curve for the muons with long track lengths (range C) rises significantly more slowly with increasing momentum. The reason for this behaviour is that muons that have travelled further in the detector must have deposited less energy upstream on average than muons that stopped short (this feature of muon energy loss is also discussed in section 6.1.3).

The bottom plot of Figure 6.28 shows the stopping power for muons in data for the 3 track length ranges (A, B and $\mathrm{C}$ ). The features observed in the $\mathrm{MC}$ (middle plot) are also seen in data: the muons that have travelled furthest (range C) in data are seen to have deposited significantly less energy upstream in the detector than those muons that stop short (range A). Another important point to make is that the Bethe-Bloch curve for range A does not rise as high in data as in MC. This is because in range A of the MC track length distribution there are only on-momentum muons. Whereas, in range A of the data track length distribution there are significant numbers of off-momentum muons as well. More precisely, in range $\mathrm{A}$ in data there are off-momentum muons that have travelled further than they would be expected to on average. The implication of this is that the off-momentum muons which do travel far enough to be in range A are those which have a less steeply rising Bethe-Bloch curve. The consequence of the off-momentum muons being present in range A of the data track length distribution is that the Bethe-Bloch curve does not rise as steeply as the corresponding distribution in $\mathrm{MC}$, which is what is seen in the middle and bottom plots of Figure 6.28.

Consider again the disagreement of the data with both the Bethe-Bloch and the MC 


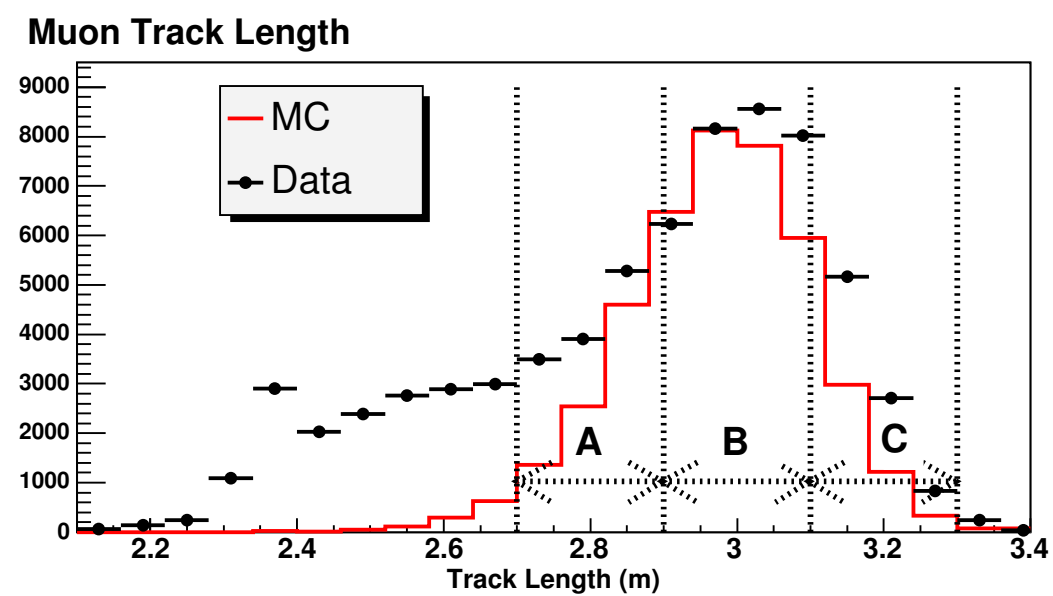

\section{Stopping Power for Muons in Polystyrene Scintillator (MC)}

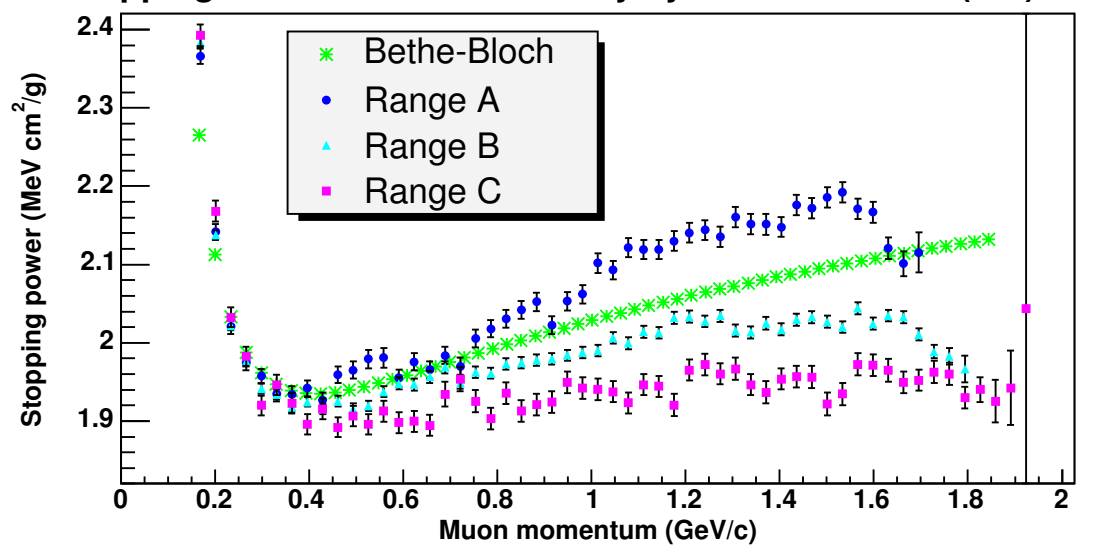

\section{Stopping Power for Muons in Polystyrene Scintillator (Data)}

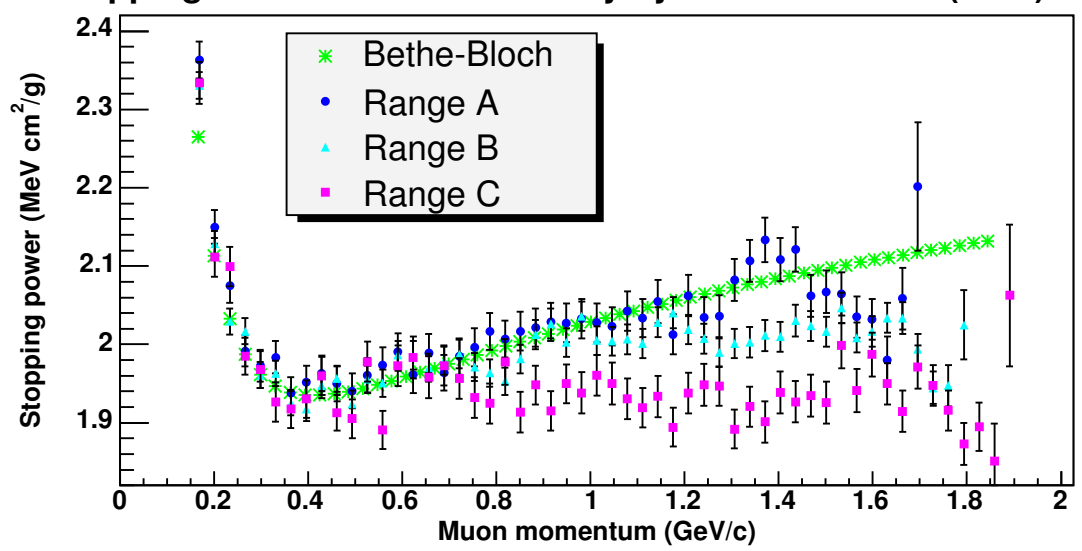

Figure 6.28: Spread in muon track length and its effect on stopping power at the Calibration detector. The top plot shows the distribution of muon track lengths. Data is shown with the black points and MC with the solid red line. The peak in the distribution is split into three separate ranges, labelled A, B and C. The middle plot shows the stopping power for muons in polystyrene scintillator from a Bethe-Bloch calculation and as measured in MC for the three separate ranges. The blue circles, turquoise triangles and purple squares show the stopping power for ranges $\mathrm{A}, \mathrm{B}$ and $\mathrm{C}$ respectively. The green stars show the stopping power obtained from a Bethe-Bloch calculation. The bottom plot shows the same as the middle plot except data instead of $\mathrm{MC}$ is used. In the middle and bottom plots the data and $\mathrm{MC}$ curves were normalised to the Bethe-Bloch calculation. 
above $\sim 1 \mathrm{GeV} / \mathrm{c}$ (as shown in Figure 6.27). It is most likely that the disagreement is caused by off-momentum muons in the test-beam. The muons are initially selected by requiring the event length to be $>40$ planes, which explains the abrupt fall off in the track length distribution for data below $\sim 2.35 \mathrm{~m}$ (the track length plotted in Figure 6.28 excludes planes on the end of a track which are determined to be cross-talk, hence there are a few events below $\sim 2.35 \mathrm{~m}$ ). The important point is that wherever the event length cut is placed there will be muons that are just accepted and some that are just rejected. The differences in the Bethe-Bloch curves for the different ranges of muons means that a bias is incurred when making an event length cut. To further show that it is likely to be the off-momentum muons in the test-beam causing the disagreement between data and $\mathrm{MC}$ in Figure 6.27, a similar plot is made where the effect of the off-momentum muons is minimised. The effect of the off-momentum muons can be minimised by only considering muons close to the peak of the track length distribution. In this region the ratio of off-momentum muons to on-momentum muons is lowest. Figure 6.29 is a plot of the muon stopping power in polystyrene scintillator vs. momentum except this time the muons are required to have a track length in range B in both MC and data, thus minimising the effect of the off-momentum muons. The agreement between MC and data is significantly better than that shown in Figure 6.27. The fact that the data and MC agree in Figure 6.29 suggests that the cause of the disagreement in Figure 6.27 is not to do with, say, calibration or reconstruction but instead is due to the off-momentum muons.

The systematic effect of the difference between data and both MC and the Bethe-Bloch calculation (as shown in Figure 6.27) on the MEU number is small. The track window samples the muon between $0.5 \rightarrow 1.1 \mathrm{GeV} / \mathrm{c}$, whereas the differences are seen above $1 \mathrm{GeV} / \mathrm{c}$. In addition, the deviations between data and $\mathrm{MC}$ are themselves small, being only $1-2 \%$. A systematic error on the MEU value, due to the differences observed, is estimated to be $\pm 0.25 \%$. The cause of the differences is believed to be due to off-momentum muons. To conclusively show that it is the off-momentum muons a MC sample with a realistic spectrum of off-momentum muons needs to be used. However, such a spectrum has not been generated or used for this thesis since the impact of the off-momentum muons on the MEU value is 


\section{Stopping Power for Muons in Polystyrene Scintillator}

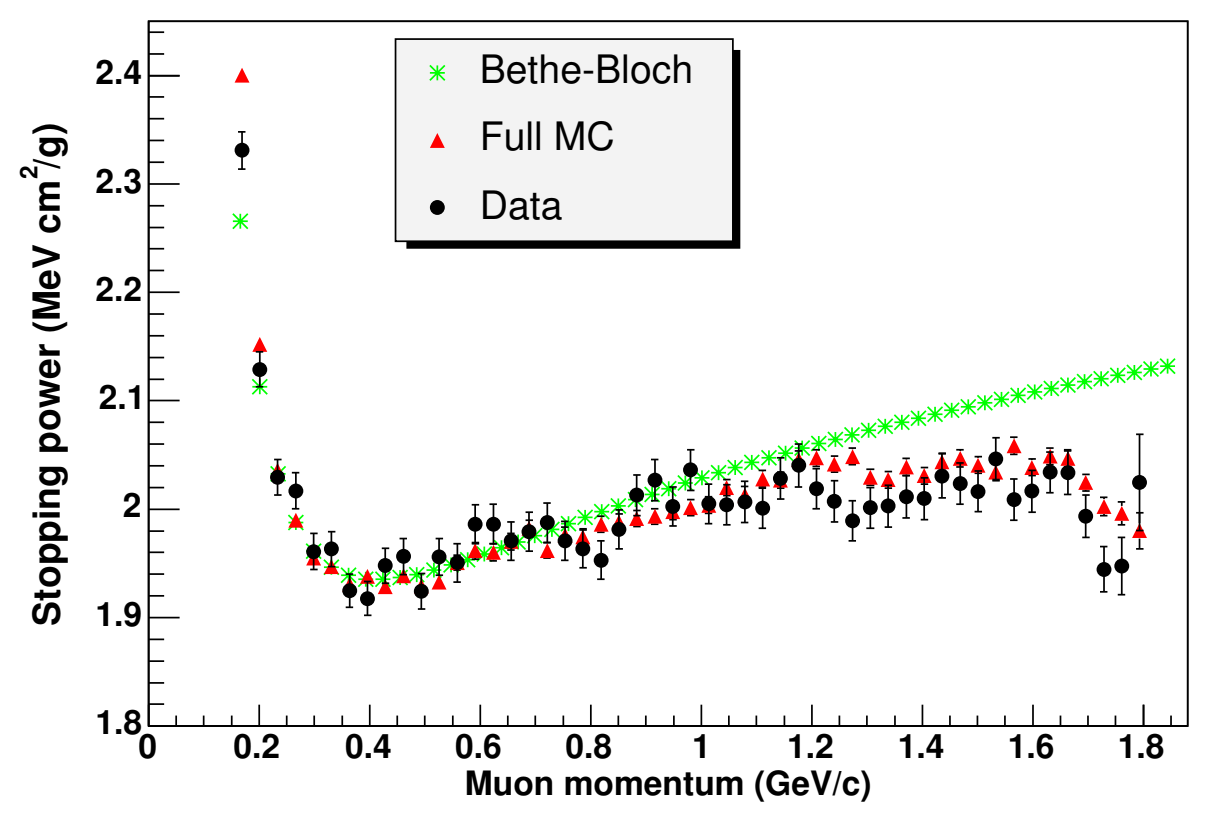

Figure 6.29: Bethe-Bloch curve for the Calibration detector with restricted muon track length. The stopping power calculated using the Bethe-Bloch equation is shown with the green stars, the measurement of the stopping power in data and MC is shown with the black circles and red triangles respectively. The data and $\mathrm{MC}$ muons were required to stop in the specific range of track lengths labelled as "B" in Figure 6.28.

small.

\subsection{Performing the Relative Calibration in MC}

As a powerful cross-check, the relative calibration is performed between the Calibration detector and the Far detector using MC simulation data. Performing this cross-check simultaneously evaluates the different components of the entire relative calibration procedure (e.g. that the stopping muons are reconstructed and attenuation corrected properly at different detectors). This cross-check also assures the self-consistency of the MC simulation for different detectors. 


\subsubsection{Calibration and "De-calibration" of the MC}

The calibration system for MINOS has been designed while taking into account the need to have a realistic and accurate $\mathrm{MC}$ simulation of the detector. When processing real data the calibration system starts with raw electronics ADCs, and finishes with an energy deposition in the scintillator in $\mathrm{GeV}$ (see section 4.5 for a description of the calibration chain). In contrast, when MC detector simulation data is generated the reverse process is required. The energy deposited in the scintillator (in $\mathrm{GeV}$ ) is known from GEANT and it is necessary to convert that energy, or "de-calibrate", to give raw ADCs. The detector simulation has to be able to, for example, simulate the effect of light attenuation along the length of a scintillator strip, simulate the effect of different cable lengths, simulate the different light output of scintillator strips, simulate the effect of PMT/electronics gain drifts over time, and so on. The way that much of the detector simulation is accomplished in MINOS is by using the real calibration constants in reverse. This procedure realistically reproduces the various detector effects (assuming the calibration constants are correct) and smears the detector response, hence it is called "de-calibrating".

Once the MC simulation data has been generated it is reconstructed and the intradetector calibration (e.g. strip-to-strip and attenuation correction) is applied using exactly the same algorithms that are used on real data. At the time of writing, the calibration of the MC data is perfect since exactly the same constants are used both to de-calibrate and then calibrate again. However, it is possible for energy to be "lost" in the current procedure. This means that the MC simulation has to have an initial, empirical tuning. Both the cause of the losses and the tuning are described in the next section.

\subsubsection{Tuning the MC Detector Simulation "light level"}

In order to generate the appropriate detector response ("light level") to a given energy deposition it is necessary to know the conversion factor between the MEU unit and GeVs. In other words, it is necessary to know the absolute energy scale of the muons used to 
perform the MEU calibration. In principle, the conversion factor between MEUs and GeVs can be extracted from the GEANT MC simulation using the truth information. However, due to the way various effects are simulated in the MINOS detector MC the procedure for obtaining the GeVs-per-MEU conversion factor is not quite so straightforward.

A number of effects cause energy deposited in the scintillator to be effectively lost, i.e. not all the energy gives rise to scintillator "light" production in the MC. The primary reason for energy loss is Birks' suppression [23], which is a saturation effect related to the ionisation density (and hence amount of scintillation) that can be obtained in a scintillator. The $\mathrm{dE} / \mathrm{dx}$ of the muon and its accompanying delta rays are obtained from the GEANT simulation. The MINOS MC then calculates the Birks' suppressed energy deposition

$$
E_{\text {supp }}=E\left(\frac{1}{1+k_{B} \frac{d E}{d x}}\right),
$$

where $E, d E / d x$ and $k_{B}$ are the energy deposition and ionisation density from GEANT, and the Birks' suppression factor respectively. The value of the Birks' suppression factor for the scintillator used in MINOS is approximately $0.1 \mathrm{~m} / \mathrm{GeV}$ [106]. A simplistic calculation of the Birks' suppression expected for, say, a $1 \mathrm{GeV}$ muon in the MINOS scintillator gives a value of $\sim 2 \%$. However, the delta rays produced by the muon have significantly higher $\mathrm{dE} / \mathrm{dx}$ on average, thus increasing the total Birks' suppression to approximately $4-8 \%$. In addition to the Birks' suppression, a number of other effects can cause energy to be lost in the simulation, such as cross-talk to unconnected pixels on a PMT. However, these other effects generally only have an effect of the order of $1 \%$.

The consequence of the energy losses in the MC is that the detector response in the MC simulation has to be empirically tuned. It is possible to achieve a tuned detector simulation using any type of particle but it is most consistent to use stopping muons since they were used to determine the MEU value. The tuning procedure developed for this thesis is as follows. A starting value of the GeVs-per-MEU conversion factor is taken as $0.0019 \mathrm{GeV} / \mathrm{MEU}$, since this is close to what is extracted from GEANT. A sample of $\sim 50000$ stopping muons is generated using GEANT and passed through the MC detector simulation. These MC 
stopping muons are then reconstructed and calibrated using exactly the same algorithms as used on real data. Finally, a MEU value for the MC stopping muons is extracted. If the MEU value measured for the MC stopping muons is higher (lower) than the value used in the de-calibration then the GeVs-per-MEU conversion factor is increased (decreased) proportionally. This procedure is repeated until the value extracted from the MC is the same as the value used in the de-calibration process.

\subsubsection{Results}

The combined self-consistency of the MC detector simulation and the relative calibration procedure is established as follows. The same tuned value for the GeVs-per-MEU conversion factor is used in the generation of stopping muons in both the Far detector and in the Calibration detector for the three run periods. Providing that the reconstruction and calibration procedures are properly applied to the MC stopping muons in all detectors then the value of a MEU extracted for each detector should match that used in the de-calibration for each detector.

Table 6.8 shows the MEU number extracted from MC stopping muons in the Far detector and the 3 run periods of the Calibration detector. The table also shows the expected MEU value (used in the de-calibration process) and the percentage deviation of the measured value from the expected value. It can be seen that the Far detector, Calibration detector T11 '02 and Calibration detector T7 '02 all have the same deviation $(\sim 0.4 \%)$ within statistical errors. In contrast, the Calibration detector $\mathrm{T} 7$ '03 has a deviation of $-1.19 \%$ from the expected value and a deviation of $1.19 \%+0.4 \% \simeq 1.6 \%$ from the Far detector and the other two Calibration detector run periods.

\subsubsection{Discussion of Results and Conclusion}

The agreement between the measured MEU value and the expected MEU value in the MC for the Far detector and the 2002 Calibration detector run periods is extremely good. These 


\section{Detector}

Far det.

Calibration det. (T11 '02)

Calibration det. (T7 '02)

Calibration det. (T7'03)
Measured MEU

$507.4 \pm 0.4$

$573.2 \pm 0.5$

$630.9 \pm 0.5$

$589.9 \pm 0.5$
Expected MEU

505.0

571.0

628.0

597.0
Deviation

$+0.48 \% \pm 0.08 \%$

$+0.39 \% \pm 0.09 \%$

$+0.46 \% \pm 0.08 \%$

$-1.19 \% \pm 0.08 \%$

Table 6.8: Results of relative calibration in MC. The measured and expected MEU value for MC stopping muons in the Calibration detector and Far detector are detailed. The deviation of the measured from expected values are given.

run periods all show the same offset $(\sim 0.4 \%)$ to within statistical errors (this offset could easily be reduced to zero by fine tuning the value of the GeVs-per-MEU conversion factor). In contrast, the discrepancy between the 2003 Calibration detector run period and the Far detector is $1.6 \%$, which is a significant deviation.

The two 2002 Calibration detector run periods used the same instrumentation (PMTs and electronics) as the Far detector. Whereas, the 2003 Calibration detector run period used Far detector instrumentation to read out one side of the detector and Near detector instrumentation to read out the other side of the detector. At the time of writing, work is underway to understand the systematic differences in detector response that arise because of the differences between the Near detector and Far detector instrumentation [76]. This work highlights two effects that can cause energy to be lost in the Near detector instrumentation w.r.t. the Far detector instrumentation.

The first effect occurs because the Far detector electronics integrates charge over a period of $\sim 400$ ns whereas the Near detector electronics integrates charge over a sequence of 19 ns periods, or "buckets". Sparsification of the data occurs at about $1 / 5$ th of a photo-electron. This means that in the Far detector electronics, energy is rarely lost since a muon on average produces $\sim 4$ photo-electrons. However, in the Near detector electronics the sparsification is done for each 19 ns bucket individually. Thus, when an event occurs near a bucket boundary it is possible for a fraction of the signal to be sparsified away.

The second effect that can cause energy to be lost is "invisible cross-talk" in the MA PMTs of the Near detector. Only 48 of the 64 pixels on a Near detector MA PMT are connected to scintillator strips in the Calibration detector. The remaining 16 pixels are unconnected, 
thus any charge that cross-talks to these pixels is effectively "invisible" - it is not included in the sum of the energy deposition. The cross-talk models in the MC detector simulation work by redistributing a fraction of the charge on one pixel to the neighbouring pixels according to pre-determined probabilities. This means that some fraction of the energy deposited in every event gets lost.

These two effects occur in both data and the MC simulation. However, when a comparison is made between the response of the Near detector and Far detector instrumentation in MC this effect shows up as a difference in absolute response. The only way that this effect can be observed in data is by, for example, taking special runs without sparsifying the electronics buckets, which is difficult experimentally.

The size of the sparsification effect is estimated to cause a loss of energy of $\sim 2-3 \%$ in the Near detector electronics w.r.t. the Far detector electronics for the energy depositions characteristic of muons [76]. The size of the "invisible cross-talk" effect can be simplistically estimated to be $16 / 64=0.25$ of the total cross-talk. Assuming the measurable cross-talk is $4 \%$ [89] of the energy deposited then potentially $4 \% \times 0.25=1.0 \%$ of the energy deposited in the Near detector instrumentation could be lost. The effect of these energy losses on the MEU value are half those stated above since the Calibration detector was read out on one side by Far detector electronics in 2003. Adding the two effects together gives a prediction of $1.0 / 2+2.5 / 2=1.75 \%$ for the expected energy loss in the Calibration detector 2003 run period w.r.t. the Far detector and the 2002 Calibration detector run periods.

The expected $1.75 \%$ extra energy loss in the Near detector instrumentation is close to the $1.6 \% \pm 0.1 \%$ loss that was measured. An exact prediction of the expected energy loss is difficult. For example, the sparsification effect has an energy dependence and the quantity of "invisible cross-talk" depends on the exact optical wiring of the fibres on the PMT face. More detailed studies of the effect of the Near detector instrumentation will be undertaken when the relative calibration is performed for the actual Near detector.

The very good agreement between the Far detector and the 2002 Calibration detector run periods demonstrate the accuracy of the relative calibration procedures and shows the self- 
consistency of the MC when the same instrumentation is used. The systematic differences between the Near and Far detectors' instrumentation have only recently come to light at the time of writing. The most appropriate techniques to compensate for the differences in the response of the instrumentation (and the MC simulation of the response) have yet to be established. It is conceivable, for example, that additional calibration steps will have to be implemented.

\subsection{Summary and Conclusions}

A precise measurement of the relative calorimetric response of the MINOS Calibration detector and Far detector using the track window technique has been accomplished. The total error on the relative calibration between the two detectors is calculated as $\sqrt{0.9^{2}+1.4^{2}}=1.7 \%$ since the systematic errors at the two detectors are uncorrelated. An error of $1.7 \%$ means that the $2 \%$ MINOS relative calibration target is accomplished.

A detailed and comprehensive analysis of all the identified systematic errors on the measurement has been undertaken. The source of systematic error associated specifically with the track window technique has been found to be only $\sim 0.5 \%$. The largest source of systematic error is shown to propagate through from the errors on the intra-detector calibration of each detector. Future improvements in the intra-detector calibration will directly lead to an improvement in the relative calibration.

Consider the potential sources of correlation in the systematic errors at the two detectors. Clearly errors such as contamination from pions or lower "off-momentum" muons only affect the Calibration detector measurements. The errors due to the spatial variations in the detector response are independent and caused by issues specific to each detector. Variations in the temporal response are also independent since they occur over very different time scales. The Far detector temperature is relatively stable over a period of days but has drifted over the 18 month period studied. In contrast the temporal fluctuations at the Calibration detector are dominated by drifts occurring over a 24 hour cycle. The angular dependence error for 
the Far detector is not present at the Calibration detector since all the muons used enter the detector horizontally.

A number of cross-checks and self-consistency checks have been performed. At the Calibration detector the known test-beam energy provides a powerful check on the consistency of the detector response between run periods. It is found that the consistency of the test-beam energy and the measured detector response is better than $2 \%$ between all three run periods considered, and that the difference between the two 2002 run periods is consistent with zero to a precision of $0.5 \%$.

Measurement of the energy deposition in the scintillator as a function of momentum has been performed at both detectors. Such measurements provide a useful cross-check that the reconstruction and calibration of the stopping muons is accurate. The measurements of energy deposition in data and $\mathrm{MC}$ are compared to the energy loss predicted by the BetheBloch equation. At the Far detector good agreement between the Bethe-Bloch curve and the measurement in data is obtained after a correction for the observed spatial variations in detector response is made. At the Calibration detector two interesting features of muon energy deposition are observed. Firstly, the production of delta rays that can penetrate several planes means that the first few planes in the detector record a lower than (naively) expected energy deposition. Secondly, the shape of the energy deposition curve depends significantly on the range of the muons from the test-beam. Consequently, the presence of the lower "off-momentum" muons in the test-beam data combined with the need to cut on muon track length to reject pions means that the measured energy deposition does not fully agree with the Bethe-Bloch curve. Although, the agreement of the GEANT MC (that does not contain off-momentum muons) with the data is good when the contamination of off-momentum muons from the test-beam is minimised, thus showing that the problem is understood.

To test the relative calibration procedures and further ensure that the reconstruction and calibration of the stopping muons is accurate in both detectors a relative calibration is performed in MC. Furthermore, the self-consistency of the MC simulation, with it's calibra- 
tion and de-calibration procedures, can be determined. The disagreement between the Far detector and the 2002 Calibration detector run periods was shown to be consistent with zero. Thus showing that the relative calibration and MC procedures are self-consistent. However, a $1.6 \%$ disagreement was discovered between the Calibration detector 2003 run period and both the two 2002 run periods and the Far detector. The reasons for the disagreement are caused by the Near detector PMTs and electronics used to read out one side of the Calibration detector in 2003. Two features of the Near detector PMTs and electronics are believed to explain the difference: cross-talk to unconnected pixels and differences in the sparsification. The procedure for correcting for these differences has not been established at the time of writing. 


\section{Chapter 7}

\section{Impact of the Relative Calorimetric Energy Calibration on MINOS}

\subsection{Neutrino Energy Reconstruction in the MINOS Detectors}

Measurement of $\Delta m_{23}^{2}$ and $\sin ^{2}\left(2 \theta_{23}\right)$ is most accurately accomplished in the MINOS experiment by comparing the Near/Far energy spectra of the muon neutrinos, as described in section 3.3.2. The visible energy of each $\mathrm{CC} \nu_{\mu}$ event is determined by reconstructing the muon and any hadronic shower produced in the interaction. The true neutrino energy is given by:

$$
E_{\nu_{\mu}}^{\text {true }}=E_{\mu}^{\text {vis }}+E_{\text {hadronic }}^{\text {vis }}+E^{\text {invis }}
$$

where $E_{\mu}^{v i s}$ and $E_{\text {hadronic }}^{\text {vis }}$ are the visible muon and hadronic energy, and $E^{\text {invis }}$ is the invisible energy absorbed by the nucleus. However, the quantity of visible energy fluctuates significantly from event to event. For example, unmeasurable fluctuations of energy loss occur in the passive detector components and also the quantity of light passing through the optical readout system fluctuates. This means that the reconstructed energy of the muon and hadronic shower can deviate significantly from the true energy. The muon energy resolution 
from a range and/or curvature measurement is estimated [107] to be

$$
\frac{\sigma_{E_{\mu}}}{E_{\mu}}=6 \%
$$

where $E_{\mu}$ is given in $\mathrm{GeV}$. The energy resolution of the hadronic shower from a calorimetry measurement was determined using the Calibration detector [68] to be

$$
\frac{\sigma_{E_{\text {hadronic }}}}{E_{\text {hadronic }}}=\frac{56 \%}{\sqrt{E_{\text {hadronic }}}}
$$

where $E_{\text {hadronic }}$ is also measured in $\mathrm{GeV}$. However, in addition to this smearing of the hadronic energy there can be an offset in the relative calorimetric energy scales between the Near and the Far detectors, which is given by

$$
E_{\text {hadronic }}^{\text {near }}=(1+\epsilon) E_{\text {hadronic }}^{\text {far }}
$$

where $E_{\text {hadronic }}^{\text {near }}$ and $E_{\text {hadronic }}^{\text {far }}$ are the calorimetric hadronic energies as measured in the Near and Far detectors, and $\epsilon$ is the relative shift in the calorimetric energy scale (due to a calibration error) between the two detectors. It is also possible for there to be an energy dependence to the value of $\epsilon$. However, in this chapter it is assumed that any such effect has been successfully calibrated out.

Figure 7.1 shows the effect of a $10 \%$ shift in the calorimetric energy scale. Three different spectra are shown: the unoscillated neutrino visible energy spectrum; the oscillated neutrino visible energy spectrum with $\Delta m_{23}^{2}=0.0025 \mathrm{eV}^{2}$ and $\sin ^{2}\left(2 \theta_{23}\right)=0.95$; and the oscillated neutrino visible energy spectrum with the same oscillation parameters but a $10 \%$ shift in the relative visible calorimetric energy scale. The migration of events from the left hand side of the peak in the oscillated-with-no-energy-shift spectrum to the right hand side of the peak in the oscillated-with-energy-shift spectrum can be clearly seen. This offset in the relative calorimetric energy scales impacts the measurement of $\Delta m_{23}^{2}$ and $\sin ^{2}\left(2 \theta_{23}\right)$. 


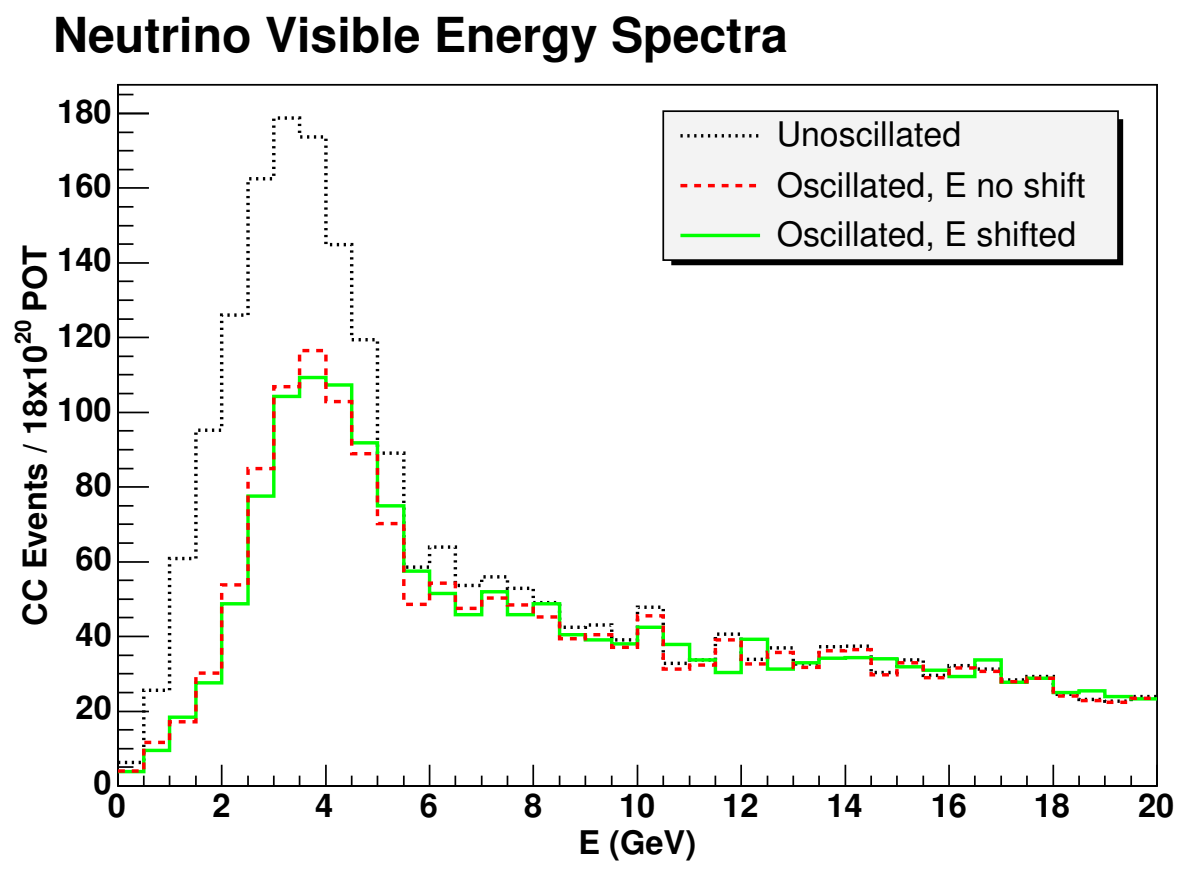

Figure 7.1: Neutrino visible energy spectra. Three different spectra are shown: the dotted black line shows the unoscillated neutrino visible energy spectrum; the dashed red line shows the oscillated neutrino visible energy spectrum with $\Delta m_{23}^{2}=0.0025 \mathrm{eV}^{2}$ and $\sin ^{2}\left(2 \theta_{23}\right)=$ 0.95; and the solid green line shows the oscillated neutrino visible energy spectrum with the same oscillation parameters but a $10 \%$ shift in the visible calorimetric energy scale.

\subsection{Impact on Oscillation Parameter Measurement}

The impact of a relative energy shift on the oscillation parameters that MINOS will measure has been studied using a MC simulation. The MC simulation fitting package was originally developed by Dr. Alfons Weber [108]. Modifications to this package have been made by the author of this thesis to study the effects of a relative calorimetric energy scale mis-calibration.

A high statistics MC sample is produced with 25000 neutrino interactions in the Far detector of which 16565 are $\mathrm{CC} \nu_{\mu}$. The energy spectrum of the neutrinos corresponds to that expected from the low-energy beam with $32 \times 10^{20}$ protons on target (POT). To simulate the detector resolution the energy of each event is smeared by equations 7.2 and 7.3. An "ideal" smeared energy spectrum is generated using all the events. This "ideal" sample is weighted according to the oscillation probability

$$
P\left(\nu_{\mu} \rightarrow \nu_{\mu}\right)=1-\sin ^{2}(2 \theta) \sin ^{2}\left(\frac{1.27 \Delta m^{2} L}{E_{\nu_{\mu}}}\right)
$$


where $L$ is set to the $735 \mathrm{~km}$ baseline of MINOS and $E_{\nu_{\mu}}$ is the true energy of the neutrino. Once the "ideal" sample for a particular oscillation scenario is established it is then used as a PDF to generate "data" samples. The total number of events used in each "data" sample is Poisson distributed according to expected running conditions of $2 \times 10^{20} \mathrm{POT} /$ year for 4 years. In order to simulate the effect of statistical fluctuations each "data" sample is constructed by randomly selecting events according to the distribution of events in the "ideal" spectrum.

The oscillation parameters are extracted from each "data" sample using a maximum likelihood technique. Only the shape information and not the normalisation is used. The probability that an event will fall into the $i$ th bin of the "ideal" spectrum is

$$
\mathrm{P}_{i}=\frac{N_{i}^{M C}}{\sum_{j} N_{j}^{M C}}
$$

where $N_{i}^{M C}$ is the number of events in the $i$ th bin and $\sum_{j} N_{j}^{M C}$ is the total number of events. The number of events in each bin of the "ideal" MC spectrum depends on the oscillation parameters:

$$
\begin{aligned}
N_{i}^{M C} & =N_{i}^{\text {unosc }} \mathrm{P}_{i}\left(\nu_{\mu} \rightarrow \nu_{\mu}\right) \\
& =N_{i}^{\text {unosc }}\left[1-\sin ^{2}(2 \theta) \sin ^{2}\left(\frac{1.27 \Delta m^{2} L}{E_{\nu_{\mu}}}\right)\right] .
\end{aligned}
$$

The likelihood function for the "data" spectrum is defined as

$$
\mathcal{L}=\prod_{i}\left(P_{i}\right)^{N_{i}^{\text {data }}}
$$

where $N_{i}^{\text {data }}$ is the number of entries in the $i$ th bin of the "data" spectrum. Taking the log of equation 7.7 gives

$$
-2 \log (\mathcal{L})=-2 \sum_{i} N_{i}^{\text {data }} \log \left(P_{i}\right)
$$


The maximum likelihood is the solution to the equations

$$
\frac{\partial(-2 \log (\mathcal{L}))}{\partial \Delta m^{2}}=0, \quad \frac{\partial(-2 \log (\mathcal{L}))}{\partial \sin ^{2}(2 \theta)}=0 .
$$

The equations in 7.9 are solved using the TMinuit minimisation package in the ROOT [109] software framework.

To simulate the effects of a relative calorimetric energy scale mis-calibration the "ideal" MC spectrum is generated after the energy of each neutrino event has been shifted according to equation 7.4. The "data" spectrum is considered to be the Far detector spectrum and the "ideal" spectrum is considered to be the extrapolated Near detector spectrum with oscillations applied. For example, an $\epsilon$ of +0.05 corresponds to the "ideal" (Near) spectrum being shifted up to higher energies with respect to the "data" (Far) spectrum. With the sign of $\epsilon$ thus defined, the best fit value for $\Delta m^{2}$ is expected to be too small when $\epsilon$ is positive, and too large when $\epsilon$ is negative.

The fractional bias on $\Delta m^{2}$ and $\sin ^{2}(2 \theta)$ calculated for each value of $\epsilon$ is defined such that a positive bias indicates that the result of the fit is larger than the true value. Thus, the fractional bias on $\Delta m^{2}$ is defined as

$$
\mathrm{B}\left(\Delta \mathrm{m}^{2}\right)=\frac{\Delta \mathrm{m}_{\text {fit }}^{2}-\Delta \mathrm{m}_{\text {true }}^{2}}{\Delta \mathrm{m}_{\text {true }}^{2}}
$$

and the fractional bias on $\sin ^{2}(2 \theta)$ is defined as

$$
\mathrm{B}\left(\sin ^{2}(2 \theta)\right)=\frac{\sin ^{2}(2 \theta)_{\mathrm{fit}}-\sin ^{2}(2 \theta)_{\text {true }}}{\sin ^{2}(2 \theta)_{\text {true }}},
$$

where the subscripts "fit" and "true" indicate which value was obtained from the MC truth and which was obtained from the likelihood fitting routine.

Figure 7.2 shows the fractional bias on $\Delta m^{2}$ as the relative calorimetric energy scale is shifted according to equation 7.4. In the top plot, the bias on $\Delta m^{2}$ is shown for 4 different values of $\Delta m^{2}$, ranging from $0.002 \rightarrow 0.005 \mathrm{eV}^{2}$ with $\sin ^{2}(2 \theta)=0.95$. In the bottom plot, 
the bias on $\Delta m^{2}$ is shown for 3 different values of $\sin ^{2}(2 \theta)$, ranging from $0.9 \rightarrow 0.99$ with $\Delta m^{2}=0.0025 \mathrm{eV}^{2} .500$ "data" spectra were generated for each combination of the oscillation parameters. However, each "data" spectrum was re-used for the 11 different fractional energy shifts to reduce the number of "data" spectra required.

There are a number of interesting observations to make about the results shown in Figure 7.2. Firstly, as expected, the size of the bias changes with the fractional energy shift. However, the rate at which the bias changes with the fractional energy shift is markedly higher for low values of $\Delta m^{2}$. In contrast, the different values of $\sin ^{2}(2 \theta)$ shown do not so drastically affect the rate at which the bias changes with the fractional energy shift. It is also interesting to see that the bias is asymmetric with respect to the fractional energy shift. A shift upwards in energy does not bias the value of $\Delta m^{2}$ by as much as a shift downwards in energy of the same magnitude. Lastly, the maximum likelihood fit has a bias to higher values of $\Delta m^{2}$ even with no energy shift, and the magnitude of this bias depends on the values of $\Delta m^{2}$ and $\sin ^{2}(2 \theta)$. This bias is due to the statistical fluctuations introduced in each "data" sample. It should be noted that because the same "data" sample is used for all 11 possible energy shifts the bias does not affect the results of this analysis. A simpler analysis that just shifted the "ideal" spectrum w.r.t. itself yielded the same slopes as shown in Figure 7.2 (but with no bias when there was no energy shift, as expected). Clearly the presence of a bias is a serious issue should the fitting process described in this thesis be used to actually determine the value of $\Delta m^{2}$ and improvements would have to be made to remove the bias. However, for the purposes of determining the effect of a relative energy shift the presence of the bias is not significant. The important result to be taken from Figure 7.2 is that for $\Delta m^{2}=0.0025 \mathrm{eV}^{2}$ and $\sin ^{2}(2 \theta)=0.95$, the bias in the fitted value of $\Delta m^{2}$ changes by approximately $0.3 \%$ for each $1 \%$ calorimetric energy scale shift (for shifts between $0.95 \rightarrow 1.05$ ).

Figure 7.4 shows the fractional bias on $\sin ^{2}(2 \theta)$ as the relative calorimetric energy scale is shifted. In the top plot, the bias on $\sin ^{2}(2 \theta)$ is shown for 4 different values of $\Delta m^{2}$, ranging from $0.002 \rightarrow 0.005 \mathrm{eV}^{2}$ with $\sin ^{2}(2 \theta)=0.95$. In the bottom plot, the bias on $\sin ^{2}(2 \theta)$ is shown for 3 different values of $\sin ^{2}(2 \theta)$, ranging from $0.9 \rightarrow 0.99$ with $\Delta m^{2}=0.0025 \mathrm{eV}^{2}$. As 


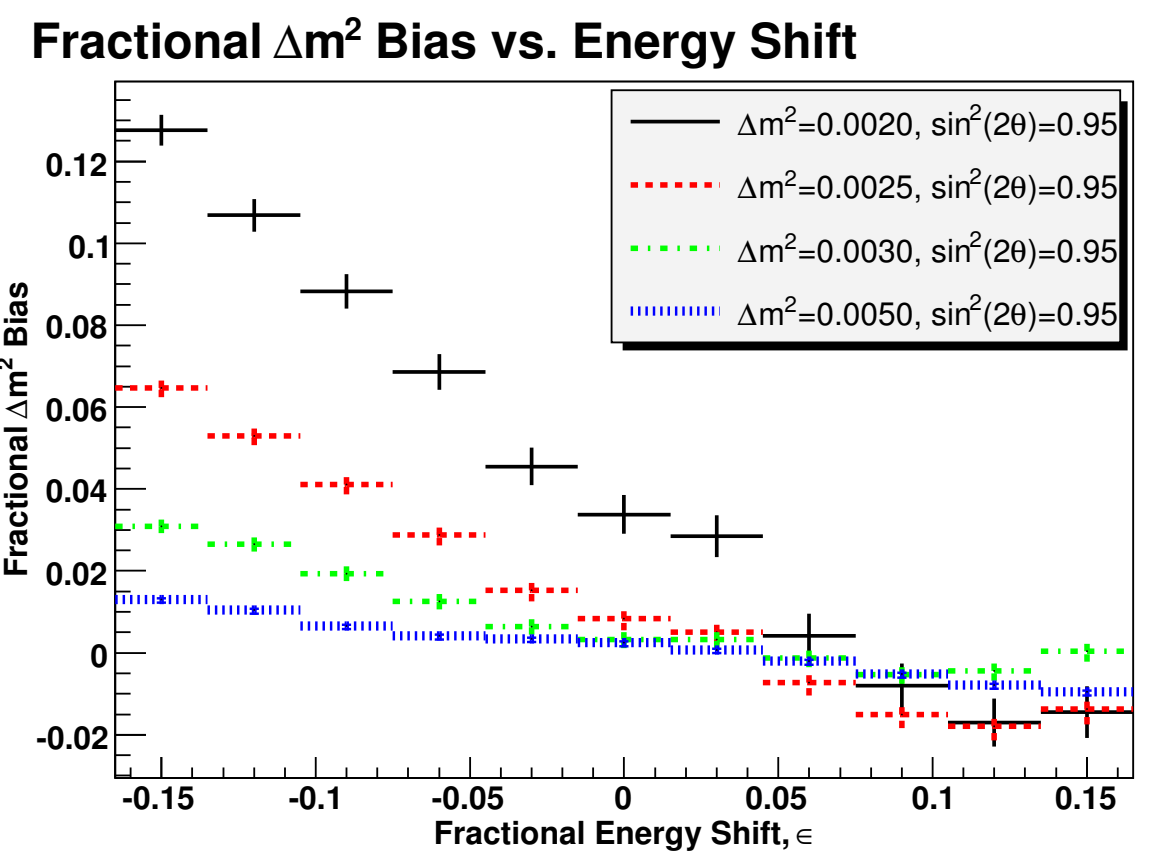

Fractional $\Delta \mathbf{m}^{2}$ Bias vs. Energy Shift

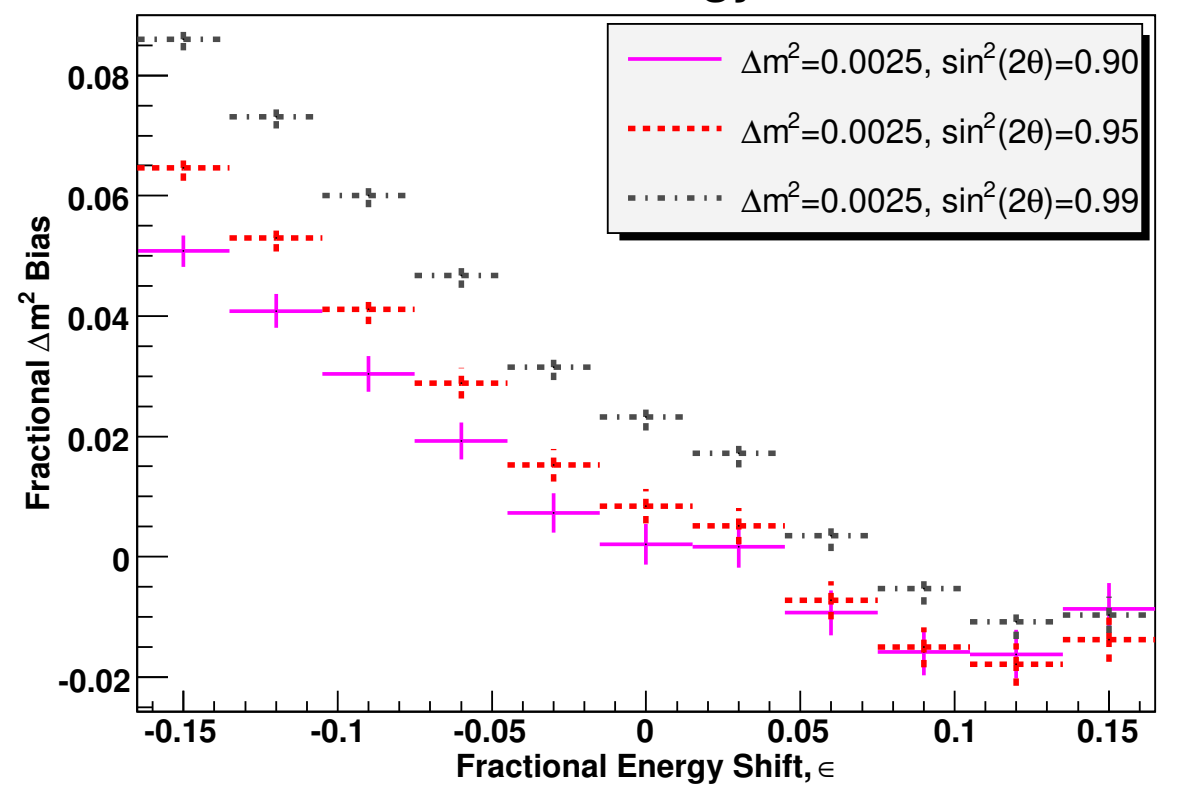

Figure 7.2: Fractional $\Delta m^{2}$ bias vs. calorimetric energy shift. Both plots show how the fractional bias on the value of $\Delta m^{2}$ obtained from the maximum likelihood fit varies with the mis-calibration of the relative calorimetric energy scale. In the top plot, the bias on $\Delta m^{2}$ is shown for 4 different values of $\Delta m^{2}$, ranging from $0.002 \rightarrow 0.005 \mathrm{eV}^{2}$ with $\sin ^{2}(2 \theta)=0.95$. In the bottom plot, the bias on $\Delta m^{2}$ is shown for 3 different values of $\sin ^{2}(2 \theta)$, ranging from $0.9 \rightarrow 0.99$, with $\Delta m^{2}=0.0025 \mathrm{eV}^{2} .500$ "data" samples were generated for each different combination of $\Delta m^{2}$ and $\sin ^{2}(2 \theta)$. 
with the study of the bias on $\Delta m^{2}, 500$ "data" samples were generated for each combination of the oscillation parameters and each spectrum was re-used for the 11 different fractional energy shifts shown.

In considering the effect of a shift in the calorimetric energy scale on the measurement of $\sin ^{2}(2 \theta)$ it is important to remember that there is a hard physical boundary at $\sin ^{2}(2 \theta)=1$. For values of $\sin ^{2}(2 \theta)$ close to one, the maximum likelihood fit cannot fluctuate to higher values. In the bottom plot of Figure 7.4 it is clear that as the value of $\sin ^{2}(2 \theta)$ approaches one the variation with fractional energy shift becomes more asymmetric. For $\sin ^{2}(2 \theta)=0.9$, the bias on $\sin ^{2}(2 \theta)$ changes by approximately $0.5 \%$ for each $1 \%$ calorimetric energy scale shift. The bias in the fit value of $\sin ^{2}(2 \theta)$ is around $1-2 \%$ with no energy shift.

In the top plot of Figure 7.4 it is clear that as the value of $\Delta m^{2}$ increases the bias on the measurement of $\sin ^{2}(2 \theta)$ due to the calorimetric energy shift reduces to almost zero. For the worst case shown, with $\Delta m^{2}=0.002 \mathrm{eV}^{2}$ and $\sin ^{2}(2 \theta)=0.95$, the bias on $\sin ^{2}(2 \theta)$ changes by approximately $0.5 \%$ for each $1 \%$ calorimetric energy scale shift.

To understand why the measurement of $\Delta m^{2}$ and $\sin ^{2}(2 \theta)$ is so much more robust for high values of $\Delta m^{2}\left(\sim 0.005 \mathrm{eV}^{2}\right)$ it is necessary to consider the shape of the oscillated neutrino spectrum. Figure 7.3 shows the neutrino visible energy spectrum for two oscillated spectra $\left(\Delta m^{2}=0.0025 \mathrm{eV}^{2}\right.$ and $\left.0.005 \mathrm{eV}^{2}, \sin ^{2}(2 \theta)=0.95\right)$ as well as the unoscillated neutrino spectrum. With $\Delta m^{2}=0.0025 \mathrm{eV}^{2}$ the events on the left hand side of the peak are most significantly oscillated away while the peak structure is still clearly visible. On the other hand, with $\Delta m^{2}=0.005 \mathrm{eV}^{2}$ the resulting neutrino energy spectrum is mostly flat. Shifting the mostly flat energy spectrum along the $\mathrm{x}$-axis according to equation 7.4 does not significantly change the shape of the distribution compared to the un-shifted distribution. In contrast, shifting a peaked spectrum along the x-axis has a significant impact when comparing to the expected shape. 


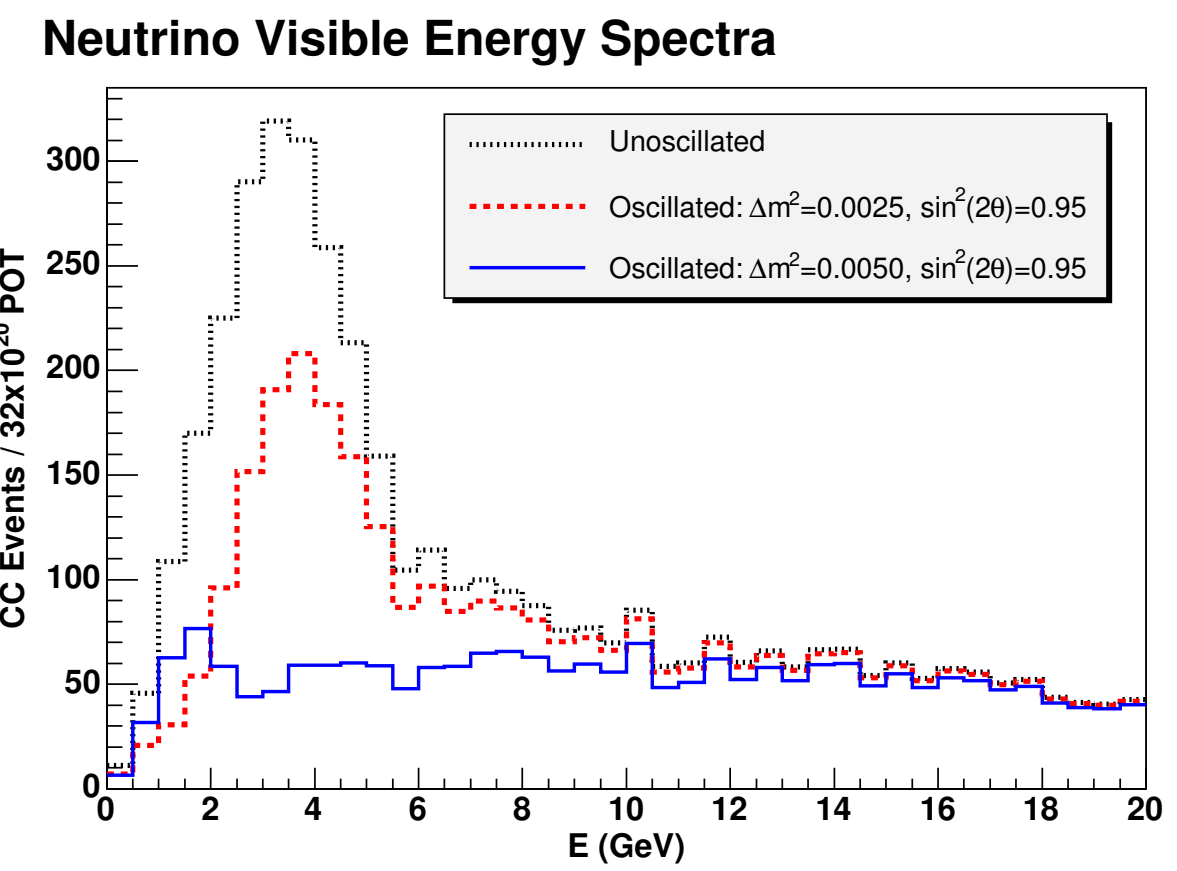

Figure 7.3: Neutrino visible energy spectra for different $\Delta m^{2}$.

\subsubsection{Summary}

The results of the maximum likelihood analysis show that the importance of the relative calorimetric energy calibration increases quite dramatically as the value of $\Delta m^{2}$ approaches the lower bound of the Super-Kamiokande $90 \%$ confidence region (around $\Delta m^{2}=$ $\left.0.0020 \mathrm{eV}^{2}\right)$. However, in contrast, should the true value of $\Delta m^{2}$ be approximately 3 sigma above the Super-Kamiokande best fit value (around $\Delta m^{2}=0.005 \mathrm{eV}^{2}$ ) then the relative calorimetric energy calibration need be far less precise to achieve the same accuracy of measurement. Although, it should also be pointed out that because the measurement of $\Delta m^{2}$ would itself be $\sim 3$ times more precise at $\Delta m^{2}=0.005$ then the error on the relative calibration would become more significant w.r.t. the other errors.

The presence of a hard physical bound at $\sin ^{2}(2 \theta)=1$ causes some difficulties in the maximum likelihood fitting procedure, it also causes an asymmetry in the effect of a calorimetric energy shift. A shift upwards of a given magnitude in the energy scale does not affect the fitted value of $\sin ^{2}(2 \theta)$ as much as a shift downwards. Similarly, but in the reverse direction, the likely position of $\Delta m^{2}$ on the left hand side of the peak of the neutrino spectra means that the bias on $\Delta m^{2}$ of a shift in the energy scale is asymmetric: shifts upwards in energy 


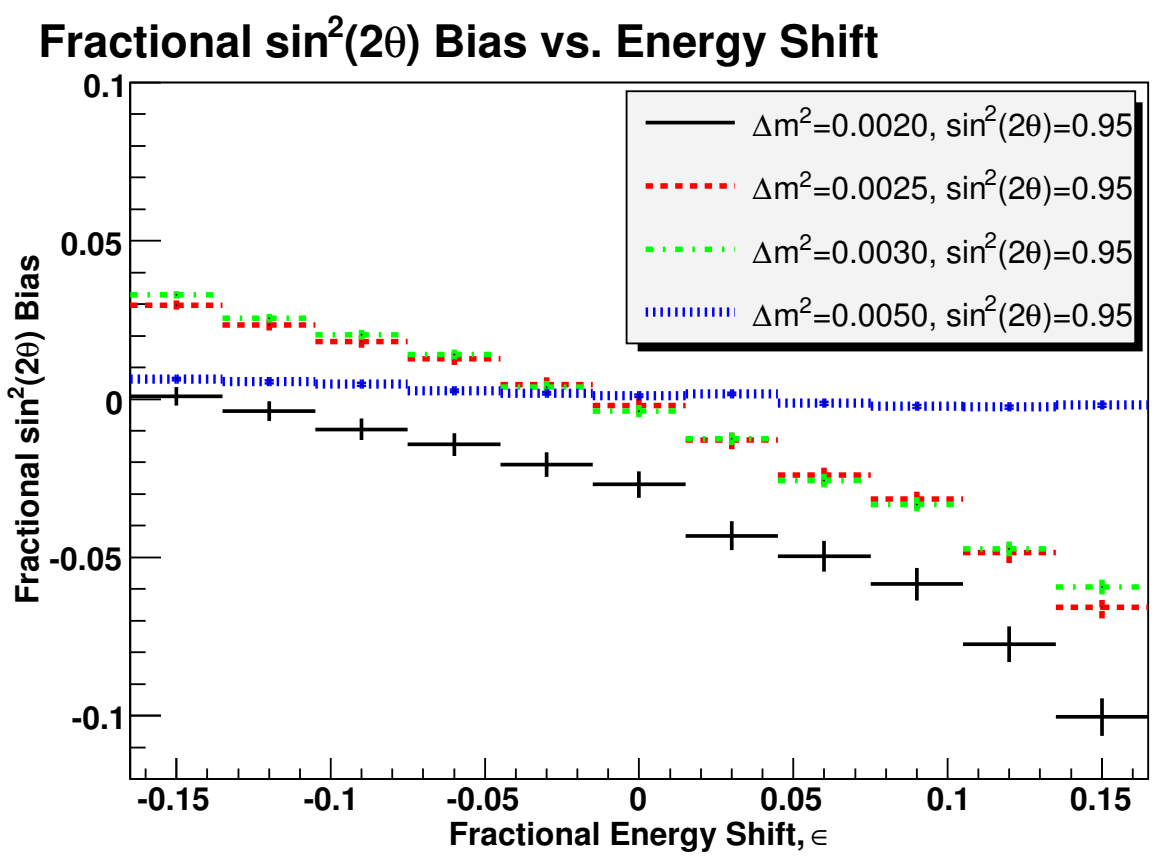

Fractional $\sin ^{2}(2 \theta)$ Bias vs. Energy Shift

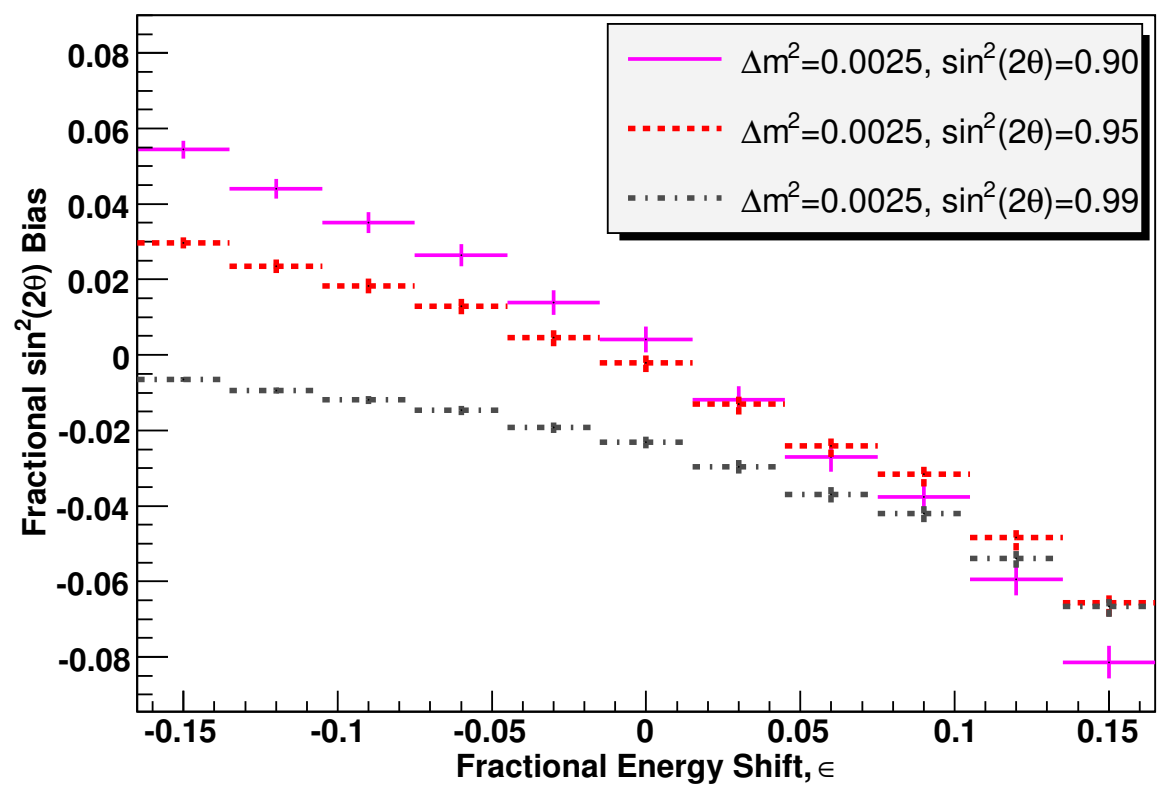

Figure 7.4: Fractional $\sin ^{2}(2 \theta)$ bias vs. calorimetric energy shift. Both plots show how the fractional bias on the value of $\sin ^{2}(2 \theta)$ obtained from the maximum likelihood fit varies with the mis-calibration of the relative calorimetric energy scale. In the top plot, the bias on $\sin ^{2}(2 \theta)$ is shown for 4 different values of $\Delta m^{2}$, ranging from $0.002 \rightarrow 0.005 \mathrm{eV}^{2}$ with $\sin ^{2}(2 \theta)=0.95$. In the bottom plot, the bias on $\sin ^{2}(2 \theta)$ is shown for 3 different values of $\sin ^{2}(2 \theta)$, ranging from $0.9 \rightarrow 0.99$, with $\Delta m^{2}=0.0025 \mathrm{eV}^{2} .500$ "data" samples were generated for each different combination of $\Delta m^{2}$ and $\sin ^{2}(2 \theta)$. 
give a smaller bias on $\Delta m^{2}$ than shifts downwards in energy.

For values of the oscillation parameters in the region favoured by Super-Kamiodande $\left(\Delta m^{2} \simeq 0.0025 \mathrm{eV}^{2}, \sin ^{2}(2 \theta) \simeq 0.95\right)$ the bias on the value of $\sin ^{2}(2 \theta)$ and $\Delta m^{2}$ changes by approximately $0.3 \%$ per $1 \%$ shift in the relative calorimetric energy scale for small shifts of $\pm 5 \%$. As discussed in section 4.1, the relative calibration target for MINOS is $2 \%$. Thus, the systematic error on $\sin ^{2}(2 \theta)$ and $\Delta m^{2}$ due to a $2 \%$ uncertainty in the relative calorimetric energy scale is $\sim 0.6 \%$ in both cases. 


\section{Chapter 8}

\section{Conclusion and Outlook}

The MINOS experiment aims to make the world's most precise measurement of the neutrino oscillation parameters $\Delta m_{23}^{2}$ and $\sin ^{2}\left(2 \theta_{23}\right)$. This will be achieved by comparing the neutrino beam spectrum measured at the Near detector with that measured at the Far detector. Measuring the spectrum of the muon neutrinos is achieved by reconstructing the energy of the muon and the possible hadronic shower produced in a charged-current interaction. The energy of the muon can be reconstructed in a relatively straightforward way by using range in the detector or curvature in the magnetic field. Reconstructing the hadronic shower is achieved using calorimetry, which requires a series of detailed calibration processes. Part of this process involved exposing the smaller Calibration detector to particles of a known energy in the CERN PS test-beams.

This thesis describes a technique developed for precisely measuring the calorimetric energy scale of the MINOS detectors. Accurate measurements of the calorimetric energy scale of the Far detector and Calibration detector have been made, which allowed the relative energy calibration to be achieved within the $2 \%$ MINOS relative calibration target. This calibration is important to MINOS for two main reasons. Firstly, measurement of the oscillation parameters by comparing the Near detector and Far detector neutrino energy spectra requires that the relative energy scale between the detectors is accurately known. Secondly, the relative calibration enables the results from the Calibration detector to be accurately 
extrapolated to the other two detectors.

\subsection{Measurement of the Detectors' Relative Response}

This thesis has detailed the accurate measurement of the relative calorimetric energy scale of the Calibration detector and Far detector. A comprehensive study of the systematic errors at both detectors has been accomplished. The response of the Calibration detector has been measured to an accuracy of $1.4 \%$ and the response of the Far detector to an accuracy of $0.9 \%$. Thus, the relative calibration between the Calibration detector and the Far detector has been achieved to an accuracy of $\sqrt{0.9^{2}+1.4^{2}}=1.7 \%$. Measurement of the Near detector's response will be performed using the methods described in this thesis once the full intra-detector calibration is available.

To achieve this accurate relative calorimetric energy calibration a new "track window" technique of using stopping muons was developed and used to provide the results in this thesis. The systematic error associated specifically with the "track window" technique has been shown to be only $0.5 \%$ and this could be reduced with further work.

The largest systematic errors have been found to propagate through from the errors on the intra-detector calibration. The stopping muons have been used to examine in detail the spatial and temporal variations of the Far detector response. Spatial variations in response have been observed within the fiducial volume. However, the effect of these variations on a neutrino measurement are shown to be only $0.6 \%$. It is expected that the Far detector intra-detector calibration will soon improve significantly.

A number of cross-checks have been made in order to ensure that the relative calibration procedures are working correctly. At the Calibration detector it is possible to perform a powerful cross-check of the measured detector response by using the known test-beam energy. The consistency of the test-beam energy and the measured detector response was shown to be better than $2 \%$ between all three run periods considered. 
Measurements of the stopping muon energy deposition curves in data and MC have been made and then compared to the expected energy loss curve predicted by the Bethe-Bloch equation. Such comparisons allow a cross-check that the reconstruction and calibration of the stopping muons used to do the relative calibration is correct. After correction for the effects of off-momentum muons from the test-beam in the Calibration detector and the spatial variation in response of the Far detector, good agreement between data and $\mathrm{MC}$ was obtained.

A relative calibration using MC data has been performed and shown to work successfully. This is an important result for several reasons. Firstly, it shows that the reconstruction and calibration of stopping muons in the Far detector and Calibration detector works well. Secondly, it verifies the self-consistency of the MC calibration and de-calibration procedure. Thirdly, it tunes the "light level" of the MC so that a realistic detector response is simulated.

\subsection{The Effect on MINOS of a Relative Miscalibration}

A study of the effect of a relative miscalibration on the measurement of the neutrino oscillation parameters has been undertaken. It was shown that for the oscillation parameters in the region favoured by the Super-Kamiokande experiment a $2 \%$ relative miscalibration would cause a $0.6 \%$ systematic error on the values of $\Delta m^{2}$ and $\sin ^{2}(2 \theta)$. Furthermore, the increasing importance of the relative calibration for lower values of $\Delta m^{2}$ was shown.

\subsection{Outlook for the future}

To further ensure that the relative calibration is accurately performed there are a number of additional measurements that could be made in the future. A source of muons that are mostly perpendicular to the detector are those produced in the rock in the front of the detector by the neutrino beam. These muons will be produced "in-spill" and hence have the advantage of being exposed to the same experimental conditions as the neutrino events being 
studied. Another way that the relative calibration can be performed is by selecting muons of a particular momentum using the magnetic field in the Near detector and Far detector. A "track window" type technique could be applied to muons of, say, $20 \mathrm{GeV}$. The relative error in the magnetic field calibration between the detectors would be offset to some extent by the approximately flat Bethe-Bloch curve for muons at $20 \mathrm{GeV}$. The measurements of stopping muons produced by the neutrino beam could be directly compared with those presented in this thesis. To compare measurements made using muons of $20 \mathrm{GeV}$ with those in this thesis, either a MC correction for the different $\mathrm{dE} / \mathrm{dx}$ could be made or the ratio of measurements at different detectors could be taken. Performing these additional measurements will be an important cross-check of the "track-window" technique and ultimately reduce the error on the values of the oscillation parameters that MINOS will determine. 


\section{Appendix A}

\section{Calibration Detector Analysis Details}

$\begin{array}{cccc}\text { Run Period } & \begin{array}{c}\text { Scintillator-TOF } \\ \text { time offset (ns) }\end{array} & \begin{array}{c}\text { Pre-event VARC } \\ \text { live time }(\mathbf{n s})\end{array} & \begin{array}{c}\text { Post-event VARC } \\ \text { live time (ns) }\end{array} \\ \text { T11 2002 } & -125 & 180 & 420 \\ \text { T7 2002 } & +30 & 280 & 320 \\ \text { T7 2003 } & +5 & 220 & 380\end{array}$

Table A.1: Calibration detector trigger times and VARC live times. The time offset between the TOF system trigger time and the time of the scintillator hits is listed for the different run periods. Also included are the VARC (VA Readout Controller: a DAQ electronics board that is read out using the VME bus) live times before and after the average beam event in the different run periods. The total VARC live time was 600 ns in each case. In 2003 the Near detector electronics was used to readout one side of the Calibration detector. The total live time of the Near detector electronics was approximately $400 \mathrm{ns,}$, with approximately $40 \mathrm{~ns}$ of live time coming before the average event and the rest after. 


$\begin{array}{ccccc}\text { Run Period } & \begin{array}{c}\text { Momentum } \\ \text { of } \mu^{+}(G e V / c)\end{array} & \text { Run number } & \begin{array}{c}\text { Momentum } \\ \text { of } \mu^{-}(G e V / c)\end{array} & \text { Run number } \\ \text { T11 2002 } & 1.4 & 40709-40711 & 1.4 & 40760 \\ \text { T11 2002 } & 1.6 & 40817 & 1.6 & 40878-40880 \\ \text { T11 2002 } & 1.8 & 40616-40620 & 1.8 & 40763-40765 \\ \text { T11 2002 } & 2.0 & 40715-40717 & 2.0 & 40722-40724 \\ \text { T7 2002 } & 1.8 & - & 1.8 & 50635-50641 \\ \text { T7 2002 } & 2.0 & 50491-50493 & 2.0 & 50454 \\ \text { T7 2003 } & 1.4 & 70567,70774,70888 & 1.4 & 70573,70783 \\ \text { T7 2003 } & 1.6 & 70542,70777 & 1.6 & 70533,70786 \\ \text { T7 2003 } & 1.8 & 70570,70796,70799 & 1.8 & 70576 \\ & & 70802,70830 & & \\ \text { T7 2003 } & 2.0 & 71266,71521 & & 70485,70488,70523 \\ & & & & \\ \end{array}$

Table A.2: Runs used in the Calibration detector MEU calibration. The momenta of each run is listed. The runs for $\mu^{-}$and $\mu^{+}$are listed separately.

\begin{tabular}{|c|c|c|}
\hline Run Period & Run Number & Run condition \\
\hline T11 2002 & 40819 & Too high beam intensity \\
\hline T11 2002 & $40000-40613$ & Too high dynode threshold \\
\hline T7 2002 & $50104-50186$ & Limited PID information \\
\hline T7 2003 & $70205-70211$ & SGATE not dynode triggering \\
\hline T7 2003 & $70266-70279$ & SGATE not dynode triggering \\
\hline T7 2003 & 70404-70407 & Pre-"production" run \\
\hline T7 2003 & $70468-70478$ & Non-standard intensity \\
\hline T7 2003 & $70705-70714$ & Capid electronics errors \\
\hline T7 2003 & 70919-70951 & Non-standard beam \& TOF settings \\
\hline T7 2003 & 71384 & PMT HV lowered \\
\hline T7 2003 & 71448 & Extra steel plate \\
\hline T7 2003 & 71525 & Offset TOF position \\
\hline
\end{tabular}

Table A.3: Calibration detector runs not used due to the beam and/or detector being operated in different conditions. Some of the run numbers listed for T7 2003 are those of the first run in a run sequence; all the runs in these sequences were not used. 


\section{Bibliography}

[1] W. Pauli, letter addressed to the participants of the Tubingen conference on radioactivity (reproduced in original German with an English translation in [18]), (1930).

[2] F. Reines and C. L. Cowan, Detection of the free neutrino, Phys. Rev. 92, 830 (1953).

[3] M. Goldhaber, L. Grodzins, and A. W. Sunyar, HELICITY OF NEUTRINOS, Phys. Rev. 109, 1015 (1958).

[4] R. Davis and D. S. Harmer, Attempt to Observe the $\mathrm{Cl}^{37}\left(\bar{\nu}, e^{-}\right) \mathrm{Ar}^{37}$ Reaction Induced by Reactor Antineutrinos, Bull. Am. Phys. Soc. 4, 217 (1959).

[5] G. Danby et al., Observation of high-energy neutrino reactions and the existence of two kinds of neutrinos, Phys. Rev. Lett. 9, 36 (1962).

[6] B. T. Cleveland et al., Measurement of the solar electron neutrino flux with the Homestake chlorine detector, Astrophys. J. 496, 505 (1998).

[7] KAMIOKANDE-II Collaboration: K. Hirata et al., OBSERVATION OF A NEUTRINO BURST FROM THE SUPERNOVA SN1987a, Phys. Rev. Lett. 58, 1490 (1987).

[8] M. Winter, Determination of the number of neutrino species from the Z line shape parameters, Prepared for Workshop on Tau Lepton Physics, Orsay, France, 24-27 Sep 1990.

[9] LSND Collaboration: A. Aguilar et al., Evidence for neutrino oscillations from the observation of anti-nu/e appearance in a anti-nu/mu beam, Phys. Rev. D64, 112007 (2001).

[10] MiniBooNE Collaboration: H. L. Ray, Current status of the MiniBooNE experiment, (2004).

[11] DONUT Collaboration: T. Patzak, First direct observation of the tau-neutrino, Europhys. News 32, 56 (2001).

[12] C. Kraus et al., Latest results from the Mainz Neutrino Mass Experiment, Nucl. Phys. A721, 533 (2003). 
[13] K. Assamagan et al., Upper limit of the muon-neutrino mass and charged-pion mass from momentum analysis of a surface muon beam, Phys. Rev. D53, 6065 (1996).

[14] J. M. Roney, Review of the tau neutrino mass, Nucl. Phys. Proc. Suppl. 91, 287 (2001).

[15] B. Pontecorvo, Inverse beta processes and nonconservation of lepton charge, Sov. Phys. JETP 7, 172 (1958).

[16] A. De Santo, An experimentalist's view of neutrino oscillations, Int. J. Mod. Phys. A16, 4085 (2001).

[17] M. Kobayashi and T. Maskawa, CP violation in the renormalizable theory of weak interaction, Prog. Theor. Phys. 49, 652 (1973).

[18] P. S. Miyagawa, Muon Energy Calibration of the MINOS Detectors, DPhil thesis, University of Oxford, 2004.

[19] NOvA Collaboration: D. S. Ayres et al., NOvA proposal to build a 30-kiloton off-axis detector to study neutrino oscillations in the Fermilab NuMI beamline, (2004).

[20] L. Wolfenstein, Neutrino oscillations in matter, Phys. Rev. D17, 2369 (1978).

[21] S. P. Mikheev and A. Y. Smirnov, Resonant amplification of neutrino oscillations in matter and solar neutrino spectroscopy, Nuovo Cim. C9, 17 (1986).

[22] J. N. Bahcall and M. H. Pinsonneault, What do we (not) know theoretically about solar neutrino fluxes?, Phys. Rev. Lett. 92, 121301 (2004).

[23] S. Eidelman et al., Review of Particle Physics, Physics Letters B 592, 1+ (2004).

[24] SAGE Collaboration: J. N. Abdurashitov et al., Measurement of the solar neutrino capture rate by the Russian-American gallium solar neutrino experiment during one half of the 22-year cycle of solar activity, J. Exp. Theor. Phys. 95, 181 (2002).

[25] GALLEX Collaboration: W. Hampel et al., GALLEX solar neutrino observations: Results for GALLEX IV, Phys. Lett. B447, 127 (1999).

[26] GNO Collaboration: M. Altmann et al., GNO solar neutrino observations: Results for GNO I, Phys. Lett. B490, 16 (2000).

[27] GNO Collaboration: T. A. Kirsten, Progress in GNO, Nucl. Phys. Proc. Suppl. 118, 33 (2003).

[28] Kamiokande Collaboration: Y. Fukuda et al., Solar neutrino data covering solar cycle 22, Phys. Rev. Lett. 77, 1683 (1996).

[29] Super-Kamiokande Collaboration: M. B. Smy et al., Precise measurement of the solar neutrino day/night and seasonal variation in Super-Kamiokande-I, Phys. Rev. D69, 011104 (2004). 
[30] SNO Collaboration: B. Aharmim et al., Electron energy spectra, fluxes, and day-night asymmetries of B-8 solar neutrinos from the 391-day salt phase SNO data set, (2005).

[31] Super-Kamiokande Collaboration: S. Fukuda et al., Solar B-8 and he p neutrino measurements from 1258 days of Super-Kamiokande data, Phys. Rev. Lett. 86, 5651 (2001).

[32] SNO Collaboration: Q. R. Ahmad et al., Direct evidence for neutrino flavor transformation from neutral-current interactions in the Sudbury Neutrino Observatory, Phys. Rev. Lett. 89, 011301 (2002).

[33] KamLAND Collaboration: K. Eguchi et al., First results from KamLAND: Evidence for reactor anti- neutrino disappearance, Phys. Rev. Lett. 90, 021802 (2003).

[34] KamLAND Collaboration: T. Araki et al., Measurement of neutrino oscillation with KamLAND: Evidence of spectral distortion, Phys. Rev. Lett. 94, 081801 (2005).

[35] J. R. Ellis, J. S. Hagelin, D. V. Nanopoulos, and M. Srednicki, SEARCH FOR VIOLATIONS OF QUANTUM MECHANICS, Nucl. Phys. B241, 381 (1984).

[36] T. Kajita and Y. Totsuka, Observation of atmospheric neutrinos, Rev. Mod. Phys. 73, 85 (2001).

[37] T. K. Gaisser and M. Honda, Flux of atmospheric neutrinos, Ann. Rev. Nucl. Part. Sci. 52, 153 (2002).

[38] C. B. Smith, Calibration of the MINOS Detectors and Extraction of Neutrino Oscillation Parameters, PhD thesis University College London, 2002.

[39] C. V. Achar et al., Detection of muons produced by cosmic ray neutrinos deep underground, Phys. Lett. 18, 196 (1965).

[40] F. Reines et al., Evidence for high-energy cosmic ray neutrino interactions, Phys. Rev. Lett. 15, 429 (1965).

[41] F. Reines et al., Muons produced by atmospheric neutrinos: Experiment, Phys. Rev. D4, 80 (1971).

[42] T. J. Haines et al., CALCULATION OF ATMOSPHERIC NEUTRINO INDUCED BACKGROUNDS IN A NUCLEON DECAY SEARCH, Phys. Rev. Lett. 57, 1986 (1986).

[43] KAMIOKANDE-II Collaboration: K. S. Hirata et al., EXPERIMENTAL STUDY OF THE ATMOSPHERIC NEUTRINO FLUX, Phys. Lett. B205, 416 (1988).

[44] The NUSEX Collaboration: M. Aglietta et al., Experimental study of atmospheric neutrino flux in the NUSEX experiment, Europhys. Lett. 8, 611 (1989).

[45] Frejus Collaboration: C. Berger et al., Study of atmospheric neutrino interactions with the Fréjus detector, Phys. Lett. B227, 489 (1989). 
[46] W. W. M. Allison et al., Measurement of the atmospheric neutrino flavour composition in Soudan-2, Phys. Lett. B391, 491 (1997).

[47] Soudan 2 Collaboration: M. C. Sanchez et al., Observation of atmospheric neutrino oscillations in Soudan 2, Phys. Rev. D68, 113004 (2003).

[48] Super-Kamiokande Collaboration: Y. Fukuda et al., Evidence for oscillation of atmospheric neutrinos, Phys. Rev. Lett. 81, 1562 (1998).

[49] W. A. Mann, Atmospheric neutrinos and the oscillations bonanza, Int. J. Mod. Phys. A15S1, 229 (2000).

[50] Super-Kamiokande Collaboration: C. Yanagisawa, Recent results from SuperKamiokande, in Frontiers in Contemporary Physics 2001, Vanderbilt University, Nashville, Tennessee, USA (2001).

[51] Super-Kamiokande Collaboration: Y. Ashie et al., Evidence for an oscillatory signature in atmospheric neutrino oscillation, Phys. Rev. Lett. 93, 101801 (2004).

[52] K2K Collaboration: E. Aliu et al., Evidence for muon neutrino oscillation in an accelerator- based experiment, Phys. Rev. Lett. 94, 081802 (2005).

[53] KARMEN Collaboration: B. Armbruster et al., Upper limits for neutrino oscillations anti-nu/mu -i anti- nu/e from muon decay at rest, Phys. Rev. D65, 112001 (2002).

[54] Y. Declais et al., Search for neutrino oscillations at 15-meters, 40-meters, and 95-meters from a nuclear power reactor at Bugey, Nucl. Phys. B434, 503 (1995).

[55] M. Apollonio et al., Search for neutrino oscillations on a long base-line at the CHOOZ nuclear power station, Eur. Phys. J. C27, 331 (2003).

[56] T2K Collaboration: Y. Hayato, T2K at J-PARC, Nucl. Phys. Proc. Suppl. 143, 269 (2005).

[57] T. Lasserre, Chasing theta(13) with new reactor neutrino experiments, hepex/0411083 (2004).

[58] G. Fogli, Neutrino Parameters, Conference proceedings from Next Generation of Nucleon Decay and Neutrino Detectors, 7-9 April 2005, Aussois, Savoie, France (to be published) .

[59] H. Murayama, updated version of a figure contained in [110], available at http://hitoshi.berkeley.edu/neutrino/.

[60] J. Hylen et al., NuMI Technical Design Handbook, Internal NuMI report (2003).

[61] J. Hylen et al., Conceptual design for the technical components of the neutrino beam for the Main Injector (NuMI), NuMI-B-285 (1997).

[62] M. Messier et al., Neutrino fluxes, hadron production, and the hadronic hose, NuMIB-700 (2000). 
[63] T. Durkin, private communication.

[64] T. Durkin and S. Robertson, Density Measurements of Carbon Steel Sample, NuMINOTE-STEEL-1073 (2005).

[65] J. Kilmer, private communication.

[66] D. Boehnlein et al., Steel Mass and Meltcode Distribution in the MINOS Far Detector, NuMI-NOTE-STEEL-1061 (2004).

[67] P. Adamson et al., Performance of a Least-Squares Based Muon Reconstruction Procedure for MINOS, NuMI-NOTE-SIM-0536 (1999).

[68] M. A. Kordosky, Hadronic Interactions in the MINOS Detectors, PhD thesis University of Texas at Austin, 2004.

[69] M. A. Barker, Hadronic Response of the MINOS Detectors, DPhil thesis, University of Oxford, 2003.

[70] P. L. Vahle, Electromagnetic Interactions in the MINOS Detectors, PhD thesis University of Texas at Austin, 2004.

[71] R. Wigmans, Calorimetry Energy Measurement in Particle Physics (Oxford University Press, 2000).

[72] J. Oliver, N. Felt, G. Feldman, A. Lebedev, and R. Lee, Design and performance of the readout system of the MINOS Far Detector, IEEE Trans. Nucl. Sci. 51, 2193 (2004).

[73] A. Belias et al., The MINOS data acquisition system, IEEE Trans. Nucl. Sci. 51, 451 (2004).

[74] MINOS Collaboration, The MINOS Detectors Technical Design Report, NuMI-L-337 (1998).

[75] G. Drake, J. Dawson, and C. Nelson, Overview of the front end electronics for the MINOS Near Detector, NuMI-L-628 (1999).

[76] A. Cabrera, Systematic Comparison of the MINOS Near and Far Detector Readout Systems, DPhil thesis, University of Oxford, 2005, to be published.

[77] R. Nichol, Calibration of the MINOS Detectors, PhD thesis University College London, 2003.

[78] The MINOS Off-line Software User's Manual Release R1.14 (2005), see also http://www-numi.fnal.gov/offline_software/srt_public_context/WebDocs/WebDocs.html.

[79] D. A. Petyt, Low $\Delta m^{2}$ sensitivity of the T-test, NuMI-S-481 (1999).

[80] D. A. Petyt, Physics potential of the three Ph2 beam designs, NuMI-L-612 (2000). 
[81] D. A. Petyt, A STUDY OF PARAMETER MEASUREMENT IN A LONGBASELINE NEUTRINO OSCILLATION EXPERIMENT, DPhil thesis, University of Oxford, 1998.

[82] D. A. Harris, Systematic Uncertainties on $\Delta m^{2}$ from Neutrino Physics, using Calorimetric Energy Reconstruction, NuMI-CONF-ANA-948 (2003).

[83] M. Diwan et al., A study of $\nu_{\mu} \rightarrow \nu_{e}$ in MINOS, NuMI-L-714 (2001).

[84] MINERvA Collaboration: S. Boyd, MINERvA: A high statistics neutrino scattering experiment in the NuMI neutrino beam, Nucl. Phys. Proc. Suppl. 139, 311 (2005).

[85] P. Adamson et al., The MINOS light injection calibration system, Nucl. Instrum. Meth. A492, 325 (2002).

[86] P. Adamson et al., On the linearity of the MINOS light-injection calibration system, Nucl. Instrum. Meth. A521, 361 (2004).

[87] P. Adamson, An LED Calibration System for the MINOS Long Baseline Neutrino Oscillation Experiment, DPhil thesis, University of Sussex, 2001.

[88] P. Adamson et al., Photoelectron Counting by Several Methods, NuMI-L-661 (2000).

[89] N. Tagg et al., Performance of Hamamatsu 64-anode photomultipliers for use with wavelength-shifting optical fibres, Nucl. Instrum. Meth. A539, 668 (2005).

[90] T. Raufer, private communication.

[91] P. Shanahan and T. N., Studies of QIE Performance with Realistic Pulse Shapes, NuMI-NOTE-ELEC-0838 (2002).

[92] G. Drake, private communication.

[93] H. Gallagher, The NEUGEN neutrino event generator, Nucl. Phys. Proc. Suppl. 112, 188 (2002).

[94] GEANT - Detector Description and Simulation Tool, CERN Program Library, Long Writeup, W5013.

[95] D. Groom, Atomic and Nuclear Properties of Materials for 292 substances: http://pdg.lbl.gov/AtomicNuclearProperties/.

[96] C. Andreopoulos, MuELoss software package: http://www-numi.fnal.gov/offline_ software/srt_public_context/WebDocs/Package_Overviews/MuELoss.html.

[97] J. Hartnell, CalDetTracker Overview: http://www-numi.fnal.gov/offline_software/srt_ public_context/WebDocs/Package_Overviews/CalDetTracker.html.

[98] R. Walczak, insightful question during viva voce examination.

[99] R. Nichol and C. Smith, Strip-to-strip Calibration at the Calibration Detector, NuMINOTE-GEN-0972 (2003). 
[100] B. J. Rebel, Neutrino-Induced Muons in the MINOS Far Detector, PhD thesis Indiana University, 2004.

[101] C. L. F. Howcroft, Atmospheric Neutrinos In The MINOS Far Detector, PhD thesis University of Cambridge, 2004.

[102] A. Blake, A Study of Atmospheric Neutrino Oscillations in the MINOS Far Detector, PhD thesis University of Cambridge, 2005, to be published.

[103] P. Adamson et al., The MINOS Calibration Detector, To be published (2005).

[104] J. J. Hartnell, CalDet gain drift analysis: http://www-pnp.physics.ox.ac.uk/ hart nell/LI/li.html.

[105] R. Lee and S. Seun, Calibration of the MINOS Detectors Using Cosmic Ray Muons, NuMI-L-694 (2000).

[106] D. Michael, private communication.

[107] D. Petyt, private communication.

[108] A. J. G. Weber, private communication.

[109] R. Brun and F. Rademakers, ROOT: An object oriented data analysis framework, Nucl. Instrum. Meth. A389, 81 (1997).

[110] Particle Data Group Collaboration: K. Hagiwara et al., Review of particle physics, Phys. Rev. D66, 010001 (2002). 